UNIVERSIDAD POLITÉNICA DE MADRID

ESCUELA TÉCNICA SUPERIOR DE INGENIEROS INDUSTRIALES

Departamento de Automática, Ingeniería Eléctrica, EleCtrónica e INFORMÁTICA INDUSTRIAL

\title{
Control Architecture of a Social Robot FOR THE NAVIGATION AND INTERACTION WITH THE ENVIRONMENT
}

PHD DISSERTATION

Author: Biel Piero E. Alvarado Vásquez, MSc.

Advisor: Fernando Matía Espada, PhD. 
Title:

Control Architecture of a Social Robot for the Navigation and Interaction with the Environment

\section{Author:}

Biel Piero Eloy Alvarado Vásquez

Master in Automatics and Robotics

Tribunal nombrado por el Mgfco. y Excmo. Sr. Rector de la Universidad Politénica de Madrid, el dia __de de 2019.

Presidente: Dr.

Secretario: $\mathrm{Dr}$

Vocal: Dr.

Vocal: Dr.

Vocal: Dr.

Suplente: Dr.

Suplente: Dr.

Realizado el acto de lectura y defensa de la tesis el dia de 2019, en el aula de la Escuela Técnica Superior de Ingenieros Industriales de Madrid.

El tribunal acuerda otorgar la calificación de:

El Presidente:

El Secretario:

Los Vocales: 


\section{Dedication}

To Blanquitha

To Bryant

To Santiago and Samuel. 


\section{Acknowledgements}

First of all, I want to thank to everyone who has been supporting me through all these years, not only in my academic life, but also in my process of becoming a better man everyday. With this dissertation finishes a journey that began in Venezuela and made come to Spain in the first place. Now I have many reasons to stay at this beautiful city called Madrid, and in this fabulous world of research.

In the first place, I'd like to thank to God, Jesus and the virgin Mary, I know they are watching me and guiding me all the time.

To my family, without their support, I would not be here. My mom who always is there for me, my brother who is the top of my best friend, my nephews whom make of me their personal big toy and my sister in law who is one of the greatest person that I know.

To Fernando, without him and his knowledge this dissertation could have not been possible to realize. Thanks for trusting in me.

To Agustín, for being the first one believing in me, for receiving me as his student while I was doing my master degree and providing me with part of his knowledge.

To Paloma, helped me a lot when I arrived to the group and I was lost. You were, are and will be a good inspiration for me to keep on the research field.

To Gema, who helped me a lot with the emotion system providing me initial ideas and for giving me more than one point of view about modern topics.

To Angel who was the responsible to build the Doris' face, to install the antennas and helped me with everything that relates to electronics. Thank you so much for your time. I owe you a lot of coffees.

To Enrique, for sharing your time with me in breakfast everyday. 
To Ramón Galán, wherever you are, I know that this dissertation has been possible thanks to your guidance, your support, for trusting me as a programmer and believing that I could make it.

To my research partners Andrés, Juan, Sofía, Jorge and all of them who shared lunch with me everyday.

To all my students who helped with a bit of grain to develop part of this creation.

To one of the greatest person that I've known, Maria, who gave good experiences, who tough me that there is still good people out there, that without her I would not be where I am right now, and supported me a lot during my firsts years of my thesis.

To Yaiza, her support, her smile, everything of her acted as a motor and made me go on and on everyday. And thank you so much for fixing my english. Now "I know kung-fu'.

To my musical friends Juan (Tortolito), Fernando (KKS), Pablo (Disperso), Miguel (Fluidos), Juanki (Torero), the another Pablo (Sinister), Percy (Playmobil), Sergio (Obiwan), Gabriel (Ventosa), everyone of them. Because without you guys and without music, life is nonsense.

To my religious friends Priest Jesus and Priest Enrique, their guidance made my connection with the all mighty something really effective.

And everyone who I do not remember right now and surely I will remember after I print this. I'm really sorry. And remembering some words of the best singer in the history: "I've taken my bows, and my curtain calls, you brought me fame and fortune and everything that goes with it, I thank you all". 
Several approaches have been made in order to create robots that can interact with people. Besides, special attention has been devoted to achieve widespread acceptance among the population. Thus, the variety of programmed robots goes from waiters, domestic helpers and personal assistants to guides that work in museums guiding people through the different areas and that explain sculptures and portraits. Doris, which belongs to this last mentioned category, is a mobile robot conceived to work in dynamic indoor environments and to interact with people. It was built as a way to upgrade Blacky and Urbano, two robots that have been developed at the Centre of Automation and Robotics at the Universidad Politécnica de Madrid in recent years.

Programming a robot is not an easy task since there are many issues that need to be solved, such as the localization of the robot, its movements, the reading of information from complex environments, the processing of some eventual conditions, the interpretation of an input and the appropriate answer to this input and so on and so forth. Once they are solved, these tasks need to work synchronized.

This dissertation presents a control architecture that involves both, hardware and software proposals, in order for Doris to be able to work as a successful tour-guide robot in museums. Therefore, five layers have been developed: a hardware, a logic, a link, a task planner and a user layer. The hardware layer communicates to the logic layer through a USB and a RS232 or Ethernet; and the remaining layers communicate with each other through pipes, shared memory, messages and sockets.

When it comes to Doris' hardware, the robot is equipped with a mobile platform. Prior to this dissertation, a skeleton placed on this platform was added to the robot in order to provide it with human appearance. Later, the head was attached to the skeleton so that interaction with the environment could be possible, as well as additional sensors such as the RFID and an omnidirectional camera, which are used for localization, navigation and interaction. 
Regarding software, there is a proposal of a client-server application which includes multiple threads which communicate with each other in order to achieve an excellent performance between internal processes and between the robot and the people.

The task planner, previously mentioned, is another proposal of this doctoral thesis, which is responsible for indicating the set of actions that Doris must perform in a museum. This actions or tasks are the set of movements that Doris needs to do in order to reach a point of interest inside the museum. A route must be traced with a designed path planner in order to reach this point of interest, and in order to make the robot follow this path, two main components must be developed: the controller and the state observer, being this last one Doris' oriented position in the plane.

Doris' localization is based on a sensor fusion, a subject that is approached with the detection of reflective beacons by means of a laser range finder, and with the detection of visual markers by means of an omnidirectional camera. The information provided along with the information contained in the semantic map is fused into an Extended Kalman Filter. Reflective landmarks are detected by using a LMS-200 laser range finder. The detection was used following certain conditions for classifying and detecting the center of the landmark by applying the Law of Sines and using it as a model in an iterative minimum squared error estimator. Visual markers are detected by using an omnidirectional camera Mobotix C25. The fiducial markers proposed for this dissertation are a matrix of 6 rows and 5 columns to avoid orientation issues, which are placed at $275 \mathrm{~cm}$ from the ground to try to avoid occlusions and get the maximum numbers of visual markers. While the camera can get up to five markers at the same time, the laser can perceive a minimum of two.

Concerning the reactive control proposed in this dissertation, two fuzzy controllers were developed. One of the controllers is a speed controller developed to move the robot from one point to another smoothly. Another controller is used in hallways, where the robot must keep in narrow areas to avoid collisions with other objects, which is similar to people's typical behaviour.

Another proposal of this dissertation is to design a semantic map of the environment that will be used for navigation. This map is subdivided into sectors and each sector reflects the points of interest where the robot must navigate. Different approaches, which are based on Dijsktra or other planners based on graphs representation, have been made to solve the navigation and planning problems.

Regarding interaction, Doris' system is based on several elements, which are the face, the arm, the emotion system, the speaking system and the lip sync system. The simpler the communication is, the better the robot will respond to the user, always having into account to avoid the uncanny valley, which is the degree of acceptance of people towards robots which are human like. 
Doris' face has the most common characteristics of a person's face, that is to say, eyes, eyelids, eyebrows, nose, lips, It is made of methacrylate and it has 20 degrees of freedom. Doris' speaking system is another proposal of this project, a system that allows Doris communication with people in the museum, thus, achieving a greater acceptance among them. This speaking system works together with the emotional system, which consists in obtaining different face expressions by means of fuzzy logic.

The remote control of Doris, which is one more proposal of this dissertation, is performed via ethernet connection. It allows to operate the robot in a local area network or in a wide area network, so that a robot located in a museum in Italy can be operated by a person located in Spain. This was a key reason for the client-server architecture to be chosen.

The client is an application which performs a request to the server (Doris). This information travels through a communication tunnel, normally ethernet, and then, the server returns the information requested to the user. The architecture should handle different clients, like clients connected with a smartphone or tablet and clients connected via web. As the applications will connect to Doris via ethernet, sockets are used except for web applications, where web-sockets are used instead.

In order to integrate al the submodules described above, a task planner is proposed, which integrates navigation, face and emotions by means of a tasks list, which is a source code of a program language developed for Doris with which the robot's user can create or modify the order in which actions are going to be performed by the robot.

Joining all the submodules into a single execution, a program for touring in the Centre of Automation and Robotics at Universidad Politécnica de Madrid has been developed. Doris initial results show that each component of the architecture produces acceptable outputs. The new language proves that modules can be merged and that Doris follows the actions (trajectories, speeches and actions based on events) specified in the program provided by the developer without making any changes in the lower layers of the architecture. 
Muchas propuestas han surgido con respecto a la creación de robots que puedan interactuar con personas. Además, se ha hecho mucho énfasis en el aspecto físico del robot para que sea bien recibido por las personas que lo rodean. Por eso, muchos robots se han diseñado para que trabajen como camareros, ayudantes domésticos, asistentes personales y hasta guías en museos, los cuales explican los retratos y esculturas. Doris, que entra en esta categoría, es un robot de interiores diseñado para trabajar en entornos complejos y para interactuar con personas. Doris es la versión moderna de otros robots, Blacky y Urbano, diseñados en el Centro de Automática y Robótica de la Universidad Politécnica de Madrid.

Para crear un robot se requieren conocimientos de mecánica, control, electrónica y programación, y programar un robot para que sea un guía turístico, no es una tarea fácil debido a que hay muchos aspectos que resolver como sus movimientos, su localización, la información que percibe del entorno, el procesamiento de ciertas condiciones que pueden cambiar en el tiempo, preguntas que pueden provenir de diferentes personas, etc. Aunque estas tareas se puedan resolver individualmente, trabajarlas en paralelo complica aún mas las cosas.

Esta tesis presenta una propuesta de arquitectura de control que cubre tanto desarrollo hardware y software para lograr que Doris trabaje de manera eficiente en un museo como guía turístico. Por lo tanto, para esta arquitectura se han desarrollado cinco capas las cuales son: hardware, lógica, enlace, planificación y usuario. La capa de hardware se comunica con la capa lógica a través de USB, RS232 o Ethernet. Las demás capas se comunican a través de otros métodos propios de los sistemas operativos como lo son pipes (tuberías), memoria compartida o sockets.

Se parte de una estructura hardware ya diseñada previamente en otro trabajo como lo es el torso de Doris, ya instalado sobre la plataforma. Sobre este torso se ha instalado una cabeza, una cámara omnidireccional, antenas RFID, altavoces y demás accesorios para 
conseguir tanto interacción como localización, navegación y planificación.

También como propuesta software se plantea un modelo cliente-servidor, en el cual se generan muchos hilos para poder trabajar los diferentes módulos paralelamente de forma síncrona y asíncrona.

Otra de las propuestas de esta tesis es un planificador de tareas, el cual permite el desarrollo de una serie de acciones dentro del museo. Estas acciones se definen como un conjunto de operaciones, tales como el movimiento del robot, su localización, la planificación de rutas, las caras que debe poner y los eventos de los que Doris debe estar pendiente, así como personas que detecta, cuando pasa por una puerta o va a entrar en un pasillo o cuando alguien le hace una pregunta.

Como la localización representa uno de los inconvenientes de un robot móvil, esta se resuelve mediante fusión sensorial implementada a través de un filtro extendido de Kalman. La fusión sensorial se hace usando un laser LMS-200 para detectar balizas reflectivas, una cámara omnidireccional para detectar marcas visuales y un mapa semántico. Con esta fusión sensorial se consigue una buena localización, con pocos milímetros de error.

De la localización depende el control reactivo del robot. Para este control reactivo se han desarrollado dos importantes controladores usando lógica difusa. Uno de ellos es un control de velocidad para que el robot vaya de manera suave a los puntos de interés del museo y el segundo controlador es para pasillos, el cual mantiene al robot en el centro de este. Esta es una conducta propia del ser humano, que camina por el centro de un pasillo y esquiva objetos o personas cuando se interponen en su camino.

La localización depende de un mapa semántico, siendo esta otra propuesta de esta tesis. El mapa semántico es construido por sectores que a su vez se subdividen en puntos de interés por los cuales se hará la navegación del robot. Esta navegación está basada en la búsqueda del mejor camino en un grafo, y para ello, los algoritmos más usados son el algoritmo de Dijsktra o el A*.

La interacción de Doris con el entorno está basada en muchos subsistemas como la cara, el brazo, el habla, la sincronización labial y el sistema de emociones. En esta tesis se propuso el diseño de una cara de apariencia humana, hecha de metacrilato con 20 grados de libertad, los cuales están distribuidos a lo largo de ojos, cejas, párpados, mofletes, y boca. Esta cara ha sido cuidadosamente estudiada para evitar rechazos por parte de los visitantes del museo. El habla, el cual es otra propuesta de esta tesis, es la que permite explicar los objetos, retratos y esculturas a las personas que están de visita. Todo esto trabajado en sinergia con la sincronización del los labios para darle una apariencia más humana al robot, pero sin llegar al rechazo.

También se han diseñado dos aplicaciones para el control remoto y local de Doris. Para el control remoto se usa una página web y para el control local, se usa una aplicación 
desarrollada en android. La idea es que con estas aplicaciones se pueda monitorizar al robot bien sea desde el mismo museo o desde otra parte del mundo. Este punto fue muy importante a la hora de establecer la arquitectura cliente-servidor.

El planificador de tareas mencionado anteriormente, es planteado en esta tesis a través de un nuevo lenguaje de programación dedicado netamente a Doris. Para esto se tuvo que proveer al lenguaje de ciertas características desde las básicas de todos los lenguajes de programación como sentencias if, while, for, declaración de variables, arrays etc, a otras más dedicadas al movimiento y las expresiones como say, move, goto, etc. y otras un poco mas avanzadas como el escuchar eventos. La versatilidad de este lenguaje de programación es la posesión de una estructura gramatical sencilla que permite crear un programa modular para cada tipo de visita turística distinta.

Ya para finalizar, se desarrollan pruebas para unir todas estas características en una misma ejecución, en el Centro de Automática y Robótica de la Universidad Politécnica de Madrid, mostrando resultados en los que cada componente puede trabajar en conjunto con todos los demás, también se puede cambiar de programa de ejecución sin necesidad de cambiar nada de la arquitectura propuesta. 
$\begin{array}{ll}\text { Contents } & \text { xv }\end{array}$

\begin{tabular}{|l|l}
\hline List of Figures & xix \\
\hline
\end{tabular}

\begin{tabular}{ll}
\hline List of Tables & xxiii
\end{tabular}

\begin{tabular}{lll}
\hline & Introduction & 1
\end{tabular}

1.1 Problem statement . . . . . . . . . . . . . . . . . . . . . . . 2

1.2 Main contributions . . . . . . . . . . . . . . . 2

1.3 Document organization $\ldots \ldots \ldots \ldots \ldots$

$2 \quad$ State of the art 9

2.1 Interactive social robots . . . . . . . . . . . . . . . . . . . 10

2.2 Localization . . . . . . . . . . . . . . . . . . . . . . . . 14

2.3 Control strategies . . . . . . . . . . . . . . . . . . . . 21

$2.3 .1 \quad$ Fuzzy logic . . . . . . . . . . . . . . . . . . . . . . . . . . . . . . . . . 23

$2.3 .2 \quad$ Fuzzy logic system . . . . . . . . . . . . . . . . . . 24

2.4 Path planning . . . . . . . . . . . . . . . . . . . . . . . . . . . . . . . . . 26

2.5 Programming languages . . . . . . . . . . . . . . . . . . . . . . . . . . . . . . . . 29

2.5 .1 Formal languages . . . . . . . . . . . . . . . . . . . . . . . . . . . 31

2.5 .2 Grammars . . . . . . . . . . . . . . . . . . . . . . . . . . . . 32

2.5 .3 Compilers and interpreters . . . . . . . . . . . . 32

2.5 .4 Lexical analysis . . . . . . . . . . . . . . . . . . . . . . . . . . . . . . . 34

2.5 .5 Syntax analysis . . . . . . . . . . . . . . . . 34

2.5 .6 Semantic analysis . . . . . . . . . . . . . . 36

3 Doris architecture $\quad 37$

3.1 Doris control scheme . . . . . . . . . . . . . . . . . . . . . 38

3.2 Hardware description . . . . . . . . . . . . . . . . . . . . . . . . . . . . . . . . . .

3.3 Software description $\ldots \ldots \ldots \ldots$. . . . . . . . . . . . . 41 


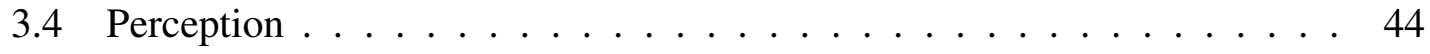

$3.4 .1 \quad$ Laser range finder $\ldots \ldots \ldots \ldots \ldots \ldots \ldots \ldots$

3.4 .2 Omnidirectional camera $\ldots \ldots \ldots \ldots \ldots$

3.4 .3 Radio Frequency Identification. . . . . . . . . . . . . . . . 48

3.5 Actuators $\ldots \ldots \ldots \ldots \ldots \ldots \ldots \ldots \ldots$

$3.5 .1 \quad$ Face controller . . . . . . . . . . . . . . . . . . 49

3.5 .2 Arm controller . . . . . . . . . . . . . . 51

3.5 .3 Text To Speech Manager . . . . . . . . . . . . . . . 53

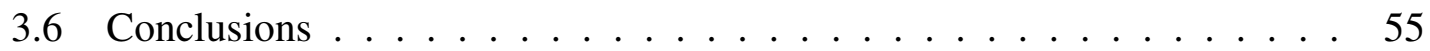

\begin{tabular}{|lll}
\hline & Localization, navigation and control system & $\mathbf{5 7}$
\end{tabular}

4.1 Localization . . . . . . . . . . . . . . . . . . . . 58

4.1 .1 Obtaining information from sensors $\ldots \ldots \ldots \ldots \ldots$

4.1 .2 Sensors error estimation $\ldots \ldots \ldots \ldots$. . . . . . . . . 68

4.1 .3 Kalman filter and integration . . . . . . . . . . . . . . . . . 74

4.2 Semantic Map . . . . . . . . . . . . . . . . . . . . . 78

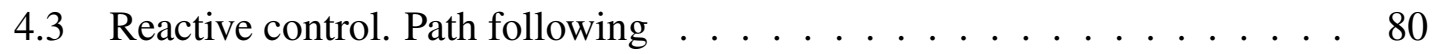

4.4 Planification $\ldots \ldots \ldots \ldots \ldots \ldots \ldots$

4.4 .1 Path planner . . . . . . . . . . . . . . . . . 83

4.4 .2 Task planner $\ldots \ldots \ldots \ldots \ldots$

4.5 Experiments and results $\ldots \ldots \ldots \ldots \ldots \ldots$

4.5 .1 Localization results . . . . . . . . . . . . . . . . . 86

4.5 .2 Reactive control results . . . . . . . . . . . . . . . . . 91

4.5 .3 Path planning results $\ldots \ldots \ldots \ldots$

4.6 Conclusions $\ldots \ldots \ldots \ldots \ldots \ldots$

$\begin{array}{lll}5 & \text { Interaction system } & 97\end{array}$

5.1 The expression system $\ldots \ldots \ldots \ldots \ldots \ldots$

5.2 Lip syncing interaction $\ldots \ldots \ldots \ldots \ldots$

5.3 The speaking system $\ldots \ldots \ldots \ldots \ldots$

5.4 The emotion system $\ldots \ldots \ldots \ldots$

5.5 Robotic arm . . . . . . . . . . . . . . . . . . . 110

5.6 Experiments and results $\ldots \ldots \ldots \ldots \ldots \ldots \ldots$

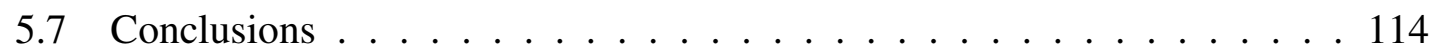

$\begin{array}{lll}6 & \text { Teleoperation system } & 115\end{array}$

6.1 Initial considerations and analysis $\ldots \ldots \ldots \ldots \ldots \ldots$

$6.2 \quad$ Design $\ldots \ldots \ldots \ldots \ldots \ldots \ldots \ldots$

6.3 Experiments and results $\ldots \ldots \ldots \ldots \ldots \ldots \ldots$

$6.3 .1 \quad$ Android application . . . . . . . . . . . . . . . . . . . . 124

6.3 .2 Web application $\ldots \ldots \ldots \ldots$

6.4 Conclusions . . . . . . . . . . . . . . . . . . . . . . . . . . . . . 129

\begin{tabular}{lll}
\hline 7 & Task planning: a new interpreter & 131
\end{tabular} 


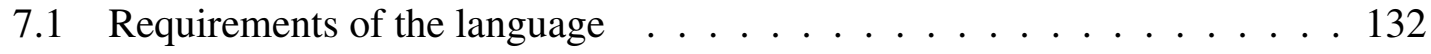

7.2 General grammar structure . . . . . . . . . . . . . . . . . . 132

7.2 .1 The if/else statement . . . . . . . . . . . . . . . . . . . 132

7.2 .2 The while statement . . . . . . . . . . . . . . . . . 134

7.2 .3 The for statement . . . . . . . . . . . . . . . . . . . 134

7.2 .4 Function definitions $\ldots . \ldots . . \ldots 135$

7.2 .5 Variable declaration . . . . . . . . . . . . . 135

7.2 .6 The say statement $\ldots \ldots$. . . . . . . . . . . . . . . 135

7.2 .7 The turn statement $\ldots \ldots \ldots$. . . . . . . . . . . 136

7.2 .8 The move statement . . . . . . . . . . . . . . . 136

7.2 .9 The goto statement . . . . . . . . . . . . . . . . 136

7.2 .10 The sizeof statement . . . . . . . . . . . . . . . . . 137

7.3 The lex () function $\ldots \ldots \ldots 137$

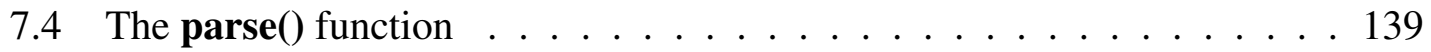

7.5 Experiments and results . . . . . . . . . . . . . . . . . . . . 139

7.6 Conclusions . . . . . . . . . . . . . . . . . . . . . . . 143

8 Conclusions and future work $\quad \mathbf{1 4 5}$

8.1 Goals reached . . . . . . . . . . . . . . . . . . . . . 146

$8.2 \quad$ Future developments $\ldots \ldots \ldots$. . . . . . . . . . . . . . . . 148

\begin{tabular}{ll}
\hline Bibliography & 151
\end{tabular} 
1.1 Social Robots . . . . . . . . . . . . . . . . . . . . . 4

1.2 Doris simplified architecture $\ldots \ldots \ldots \ldots \ldots \ldots \ldots$

1.3 Robot localization and navigation $\ldots \ldots \ldots \ldots \ldots \ldots$

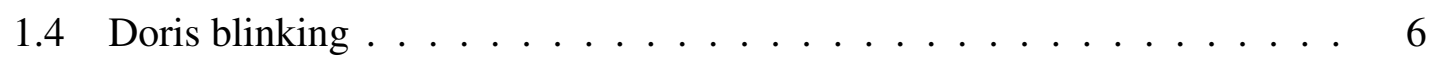

1.5 Doris webpage for teleoperation $\ldots \ldots \ldots \ldots \ldots \ldots$

1.6 Doris interpreter for task planning $\ldots \ldots \ldots \ldots \ldots \ldots$

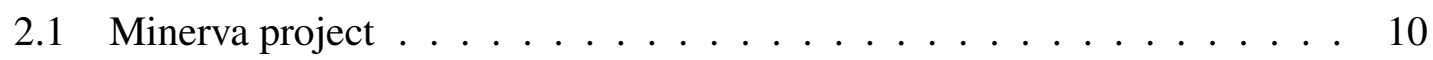

2.2 Robovie project . . . . . . . . . . . . . . . . . . . . 12

2.3 Mori's uncanny valley $\ldots \ldots \ldots \ldots$

2.4 CAR's social robot URBANO . . . . . . . . . . . . . . . . . . . 14

2.5 CAR's robot BLACKY . . . . . . . . . . . . . . . . . . . . . 17

2.6 Representation of a fuzzy set used to define environment temperature . . 24

$2.7 \quad$ Basic configuration of a fuzzy logic system $\ldots \ldots \ldots \ldots \ldots$

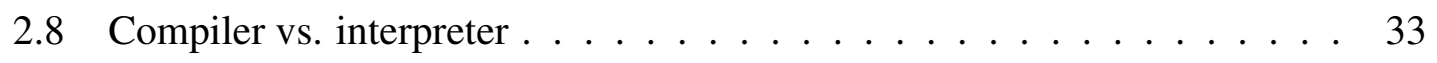

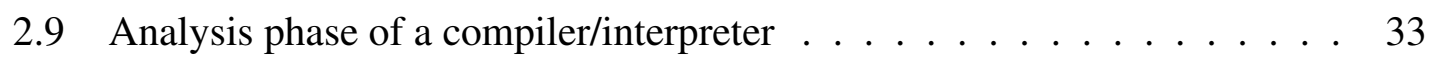

2.10 A parse tree according to the grammar $2.1 \ldots \ldots \ldots \ldots$

3.1 Software architecture of Doris $\ldots \ldots \ldots$. . . . . . . . . . . 39

3.2 Doris platform $\ldots \ldots \ldots \ldots \ldots \ldots \ldots \ldots \ldots \ldots \ldots \ldots$

3.3 Doris skeleton $\ldots \ldots \ldots \ldots \ldots \ldots \ldots \ldots \ldots \ldots$

3.4 Doris head . . . . . . . . . . . . . . . . . . 42

3.5 Class diagram of principal classes $\ldots \ldots \ldots \ldots \ldots$

3.6 Class diagram of threads $\ldots \ldots \ldots \ldots \ldots$

$3.7 \quad$ LMS200 operating principle $\ldots \ldots \ldots \ldots \ldots \ldots \ldots \ldots$

3.8 LMS200 direction of transmission and 180 degrees of scanning. . . . . 46

3.9 Omnidirectional camera operating principle $\ldots \ldots \ldots \ldots \ldots$

3.10 Mobotix C25 Omnidirectional camera . . . . . . . . . . . . . 47

3.11 Image taken by the omnidirectional camera . . . . . . . . . . 47 
3.12 Information transfer in an automatic identification system . . . . . . . . . 48

3.13 Sample of a tag used in RFID systems . . . . . . . . . . . . . . . . . . . . . . . . . . . 48

3.14 RFID System installed on Doris . . . . . . . . . . . . . . . . . . . . . . . . . . . . . . 59

3.15 Doris initial design face . . . . . . . . . . . . . . . . 50

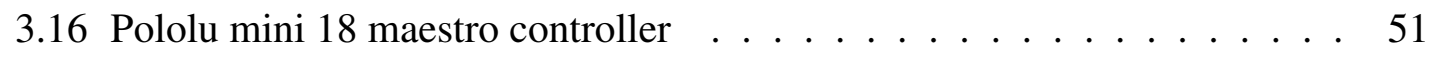

3.17 Robotic arm model . . . . . . . . . . . . . . . . . . 52

3.18 Dynamixel Motors . . . . . . . . . . . . . . . . 52

3.19 Connections between each dynamixel motor . . . . . . . . . . . . . 53

3.20 USB2Dynamixel converter . . . . . . . . . . . . . . . . . . . . . . . . . . . 53

3.21 Doris arm class diagram $\ldots \ldots \ldots \ldots$. . . . . . . . . . . . . . . . . . . . . 53

3.22 Class diagram of the text to speech manager . . . . . . . . . . . 54

3.23 Hardware used for text to speech . . . . . . . . . . . . . 55

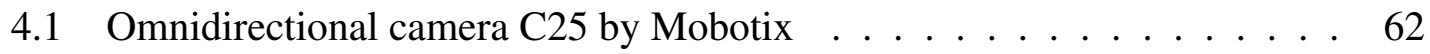

4.2 Example of a visual landmark . . . . . . . . . . . . . . . 62

4.3 Images obtained in the treatment process . . . . . . . . . . . . . . . . . . . . . . . . . 64

4.4 Reflective landmark designed by the group . . . . . . . . . . . . . . . . 65

4.5 Measurement representation obtained by the laser . . . . . . . . . . . . . . . 65

4.6 Estimation model for landmark center . . . . . . . . . . . . . . . . . 67

4.7 Real vs estimated position of reflective landmarks . . . . . . . . . . . . 70

4.8 Visual marker placed at zero degrees . . . . . . . . . . . . . . . . . . 72

4.9 Membership functions of the distance input to he speed controller . . . . 81

4.10 Membership functions of the angle input to the speed controller . . . . . 81

4.11 Linear speed output memberships function of the speed controller . . . . 81

4.12 Angular speed output membership functions of the speed controller . . . 81

4.13 Fuzzification of zones performed by the laser . . . . . . . . . . . . . . . . . 82

4.14 Each membership function of the input zones . . . . . . . . . . . . . . . . . 82

4.15 Membership functions of linear speed . . . . . . . . . . . . . . . . . . . . . 83

4.16 Membership functions of angular speed $\ldots \ldots \ldots . \ldots . \ldots 83$

4.17 Class diagram of path planner . . . . . . . . . . . . . . . . . . . . . . . . . . 84

4.18 Representations each sector connection . . . . . . . . . . . . . . . 84

4.19 Representations of the ways where Doris navigates in a sector . . . . . 85

4.20 Class diagram of task planner . . . . . . . . . . . . . . . . . . 86

4.21 Distribution of visual and reflective landmarks . . . . . . . . . . . . 87

4.22 Odometrical position of the robot vs. EKF using only LMS-200 . . . . 88

4.23 Estimated position covariance of the robot using only LMS-200 . . . . . 88

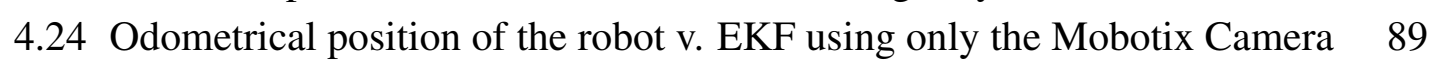

\begin{tabular}{|ll}
4.25 & Estimated position covariance of the robot using only the Mobotix Camera 90
\end{tabular}

4.26 Odometrical position of the robot versus EKF using both LMS-200 and the Mobotix Camera . . . . . . . . . . . . . . . . . 91

4.27 Estimated position covariance using both LMS-200 and the Mobotix

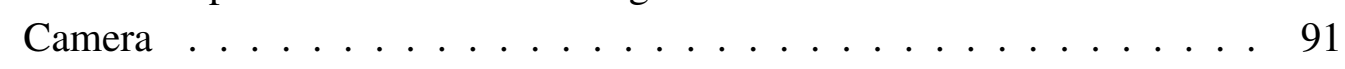

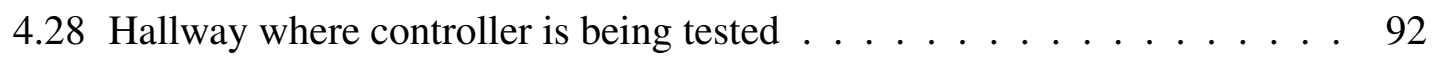


4.29 Odometrical vs EKF trajectory of the robot using both sensors . . . . . . 93

4.30 Odometrical covariance position using both sensors . . . . . . . . . . . . 94

5.1 Doris faces $\ldots \ldots \ldots \ldots$

5.2 Human-Doris mouth relation . . . . . . . . . . . . . . . . . . . 101

5.3 Lips synchronization flowchart . . . . . . . . . . . . . . . . . 101

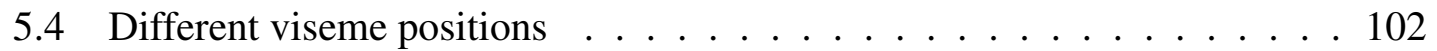

5.5 Dialog task scheme . . . . . . . . . . . . . . . . . . . . 102

5.6 Input emotion variables sample as a Fuzzy Set . . . . . . . . . . . . . . 106

5.7 Fuzzy output eyelids variable . . . . . . . . . . . . . . . . . . . . . . . . . . . . . . . . . . . . . .

5.8 Fuzzy output eyebrows variable $\ldots \ldots$. . . . . . . . . . . . . 107

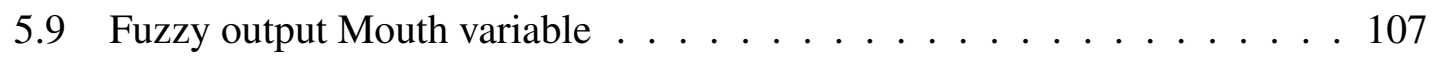

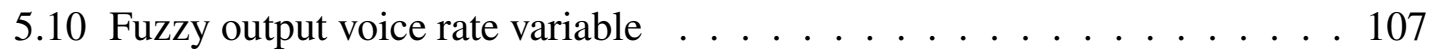

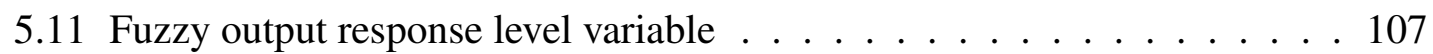

5.12 Doris robotic arm . . . . . . . . . . . . . . . . 111

5.13 Doris getting angry $\ldots \ldots \ldots \ldots$. . . . . . . . . . . . 112

6.1 Use case diagram of client application . . . . . . . . . . . . . . 117

6.2 Use case diagram of face expression section . . . . . . . . . . . . . . 118

6.3 Use case diagram of emotions section . . . . . . . . . . . . . . . 118

6.4 Use case diagram of the navigation section . . . . . . . . . . . . . . . . 118

6.5 Use case diagram of arm expression section . . . . . . . . . . . . . . . 119

6.6 Use case diagram of requesting robot ownership . . . . . . . . . . . . . . 119

6.7 Main activity class diagram . . . . . . . . . . . . . . . . . . . . . . . . . . . 120

6.8 TCP client connection class diagram . . . . . . . . . . . . . . 120

6.9 Facial expressions class diagram $\ldots \ldots \ldots$

6.10 Navigation class diagram . . . . . . . . . . . . . . . . . . . 121

6.11 Autonomous navigation class diagram . . . . . . . . . . . . . . . 121

6.12 Manual navigation class diagram . . . . . . . . . . . . . . . . . 121

6.13 General connection scheme . . . . . . . . . . . . . . . . . . 123

6.14 Initial screen of the android application . . . . . . . . . . . . . . . 124

6.15 Notification of a permission request from another user. . . . . . . . . . . 125

6.16 Android expressions screen . . . . . . . . . . . . . . . . . . 125

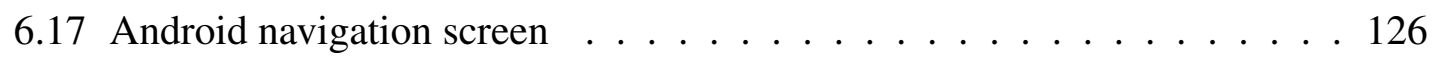

6.18 Current user settings screen . . . . . . . . . . . . . . . . 126

6.19 Expression Section . . . . . . . . . . . . . . . . . . 127

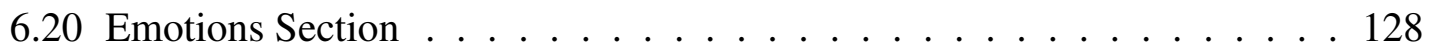

6.21 Mapping section . . . . . . . . . . . . . . . . . . . 128

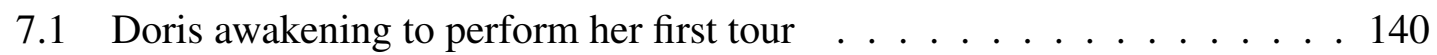

7.2 Doris moving to a point on her first tour . . . . . . . . . . . . . 141

7.3 Doris arrival to the desired point . . . . . . . . . . . . . . . . 141

8.1 Robots for interaction . . . . . . . . . . . . . . . . . . 145

Biel Piero E. Alvarado Vásquez $\quad$ Page xxi 


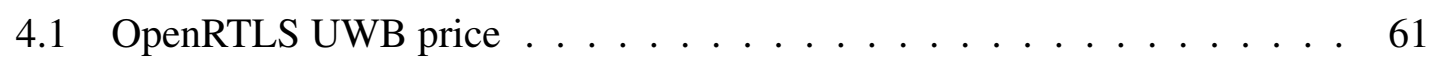

4.2 Process Noise standard deviation . . . . . . . . . . . . . . . . . . 69

4.3 Reflective landmark real position vs estimated position . . . . . . . . . . 70

4.4 Reflective landmark errors at different distances . . . . . . . . . . . . . . 70

4.5 Reflective landmark standard deviation . . . . . . . . . . . . . . . 71

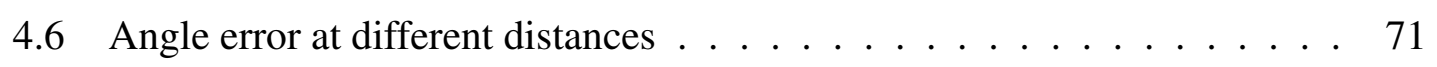

$4.7 \quad$ Angle Standard Deviation. . . . . . . . . . . . . . . . . . . . . . . . . . . . . . . . . 73

4.8 Standard deviation of RSSI and RF Phase . . . . . . . . . . . . . . . . . 73

4.9 Values of RSSI and distance in a complex environment . . . . . . . . . 73

4.10 RFID standard deviation . . . . . . . . . . . . . . . 73

4.11 Ground truth Robot position vs. Real Position . . . . . . . . . . . . . . . 94

4.12 Ground truth error . . . . . . . . . . . . . . . . . . . 95

4.13 Sensor list prices $\ldots \ldots \ldots \ldots$. . . . . . . . . . . . . . . . 96

5.1 Doris head degrees of freedom distribution . . . . . . . . . . . . . . . . . . . . . . . . 98

5.2 Humans muscles implied on speaking . . . . . . . . . . . . . . . . 100

5.3 Doris trying to get $\mathrm{Sad}$. . . . . . . . . . . . . . . . . . . 112

5.4 Doris trying to get angry $\ldots \ldots \ldots \ldots \ldots$ 


\section{Introduction}

Mobile robots are characterized by the capacity to move autonomously in an unknown or partially known environment. It is important to say that autonomy depends basically of three factors:

- Localization: where am I?

- Task planning: where do I want to go?

- Movement planning: how can I get there?

For these tasks, a sensorial system must be installed on the robot in order to provide information from the environment, and when the robot moves along, the position is always updated.

One of the different applications of mobile robots is social robotics, where the robot is designed in a half human form, where the robot interacts with people in a simple and nice form, following behaviours, patterns and social rules. For these tasks, modules performing some artificial intelligence must be contained in the social robot, including the fact that its physical appearance must be accepted in the respective environment. Socialization with people is not an easy task because humans observe the world in one way and are only able to communicate with other humans, robots have their own language which has no relation with human language.

Interactive robots work as partners, resulting that the robot needs certain degree of adaptability and flexibility to keep interaction with humans in different range of ages. These robots can have different shapes and diverse functionality. 


\subsection{Problem statement}

The main goal of this dissertation is to develop a control architecture of a social robot for the navigation and interaction with indoor environments like laboratories, museums, fair-trades, etc. For this reason some specific goals are established:

1. Locate autonomously the robot position based on different points of reference. For this, an implementation of the Extended Kalman Filter and a sensor fusion based on laser range finder, an omnidirectional camera and a radio frequency system is going to be developed according to the proposed architecture.

2. Navigate autonomously over the environment. For this purpose, a semantic map must be created so the robot can have a priori knowledge of where it is going to perform, being the planned task fully performed. For navigation fuzzy controllers will be developed.

3. Achieve an interaction with the environment. Several elements in the robot and placed in the environment. For the elements in the robot, will be a human like torso over the mobile platform, a robotic face and a robotic arm over the torso and also an emotional system based in fuzzy logic to modify the interaction with people. In the environment, radio frequency tags are placed so the robot can offer explanations or perform other actions.

4. Integrate all the subsystems created into a simple task planner, creating a robust system with the possibility to add new modules.

\subsection{Main contributions}

According to the author considerations, the principal contributions from this dissertation are:

1. Proposal of a control architecture based on a client-server application which handles several number of threads, gathering information from different sensors and executing different functions to control the interaction with the environment.

2. A localization and navigation system based on sensor fusion using a laser, an omnidirectional camera and a semantic map of the environment for localization, with the presence of tags read by an RFID system to execute different actions or for Doris being able to know who is next to her. This system also includes the navigation where fuzzy controllers are included, the first controller for path following in normal spaces and another controller for hallways. 
3. Development of an interactive system as an interface for humans capable of showing faces according to emotions (another thread that is being executed from the beginning of the application). This interaction also includes an arm to hail or to point to different directions.

4. Design and implementation of an emotion system based on fuzzy logic where an input text is provided. The text is a question or a comment formulated to Doris. This text is processed by another module of the architecture, the dialog system, that is intended to look for the proper answer to the user. This answer depends on the emotion state of Doris and the emotion state will provide a physical expression in the face and a voice tone according to the Doris' feelings.

5. Development of a teleoperation and supervisory system where a webpage and an android application were designed to control the robot.

6. Development of an integration system where a new interpreted language is made. This language allows to communicate with other submodules inside the architecture. This leads to the development of a lexer and a parser to analyze an input source program and command the actions of Doris.

\subsection{Document organization}

The organization of this dissertation is shown next by giving a briefly explanation of each chapter. The order of these chapters follows the order of how the evolution has been through the years by first performing a research of the current state of art, passing by the design and implementation and finally, concluding by showing results in publications.

The current state of research in interactive robots is described in Chapter 2. This includes the research performed by different groups in diverse fields like interactive robots (figure 1.1). Nao, Pepper, iCub, Sophia and others can be found among these type of interactive robots. Also localization and navigation techniques can also be found in this chapter along with other control architectures for social robots, path planning, and task planning. For the control architecture, the most notorious work has been performed using Fuzzy Logic, a brief explanation of this methodology can be also found in this chapter. And finally, for task planning and subsystems integration a new interpreted language was developed, a brief explanation of how compilers and interpreters are designed is provided as well. 


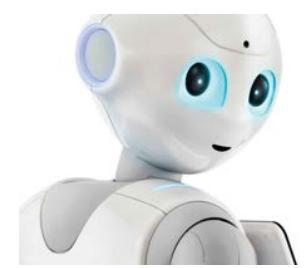

(a) Pepper

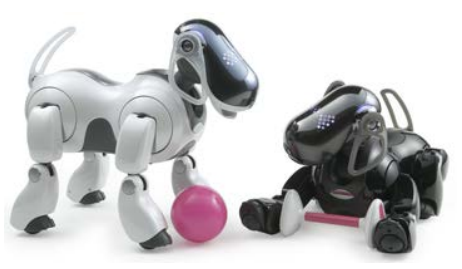

(b) Aibo

Figure 1.1: Social Robots

Doris' control architecture design and implementation are explained in Chapter 3. The chapter begins by explaining the Client-Server model implemented for the software and how the different layers of the architecture communicate with each other. The chapter continues by detailing the hardware installed and how different components communicate. For localization and navigation, new sensors were installed, so a very brief explanation of how these sensors work and operate is provided. For interaction, it is explained how the face and the arm were designed and how these elements connects with the software. Other input and output submodules are explained such as the emotion and lip syncing sub-systems. And finally the task planner layer is also explained. The reduced model of the designed architecture is shown in figure 1.2, where the inputs are the sensors, speech and the user's program that connect to the software core, and the outputs, which are working in parallel, are localization and navigation, expressions (face and arm), emotions and speech.

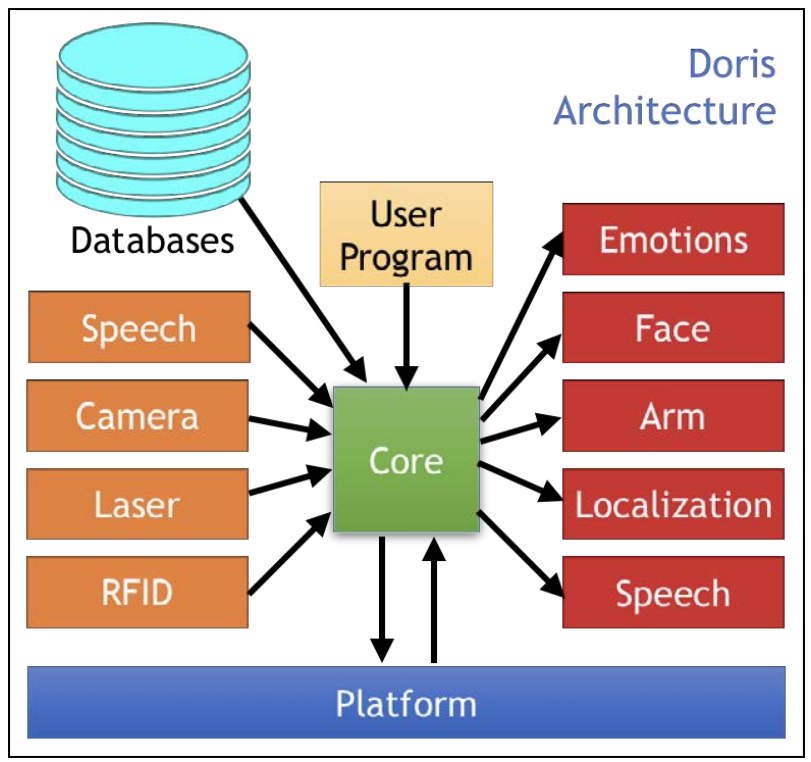

Figure 1.2: Doris simplified architecture

The localization and navigation system is fully explained in Chapter 4, which is one of the contributions of this dissertation. The localization is based on a sensor fusion using laser range finder and an omnidirectional camera combined with an Extended 
Kalman Filter as shown in figure 1.3. In this chapter, the way in which data from each sensor is processed and then sent to the localization thread is also explained in detail. Then, a detailed explanation on how to calibrate each one of the sensors by obtaining the measurement error is provided. This error is also obtained from the system odometry provided by encoders. A detailed explanation on how to implement the Extended Kalman Filter with sensor fusion is also given. The localization is achieved by integrating the information collected from sensor using a semantic map and the EFK. Then, later on this same chapter, the navigation system is detailed and how fuzzy logic helps with the path following in different environments by developing two types of controllers, one controller for normal rooms and other controller to navigate in hallways. As the map is subdivided in sectors, an $\mathbf{A}^{*}$ and a Branch and Bound algorithms are implemente to achieve navigation inside a sector and between each sector. Finally, a set of tests and conclusions are shown at the end of the chapter.

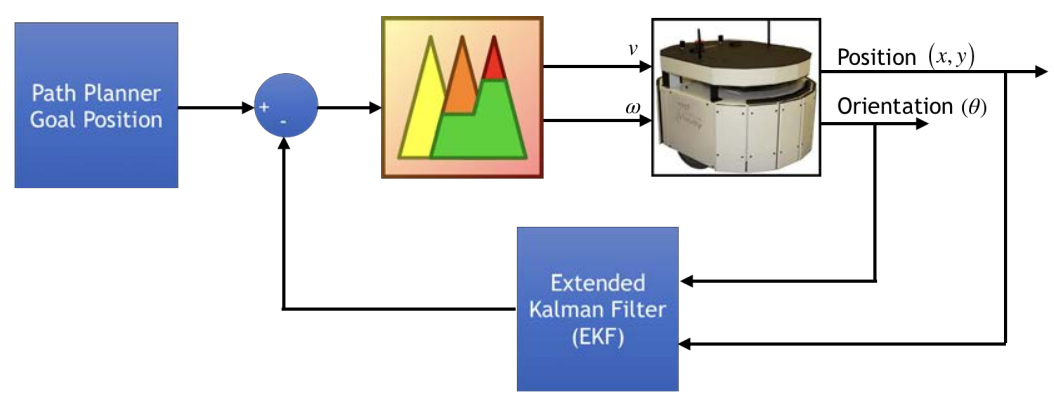

(a) Localization

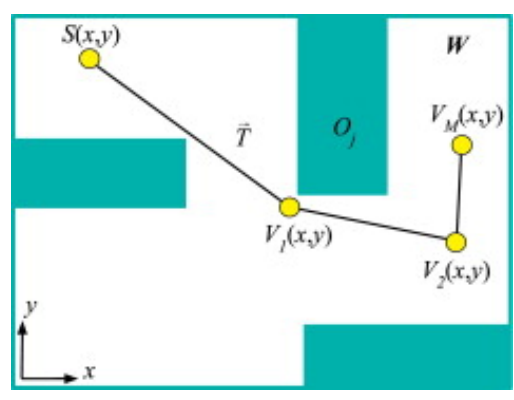

(b) Navigation

Figure 1.3: Robot localization and navigation

Rhe interaction system is fully explained in Chapter 5, which is another contribution of this dissertation. The gesture system is a thread dedicated to Doris expressions as shown in figure 1.4 where Doris is blinking an eye, the dialog system is a thread who its function is to look for answers and emit them when someone talks to Doris, and the emotional system which is another thread dedicated to vary the internal state emotion of Doris according to the given inputs. The way in which these threads communicate each other is also explained in this chapter. Finally, a robotic arm that is also used for interaction is explained as well. 


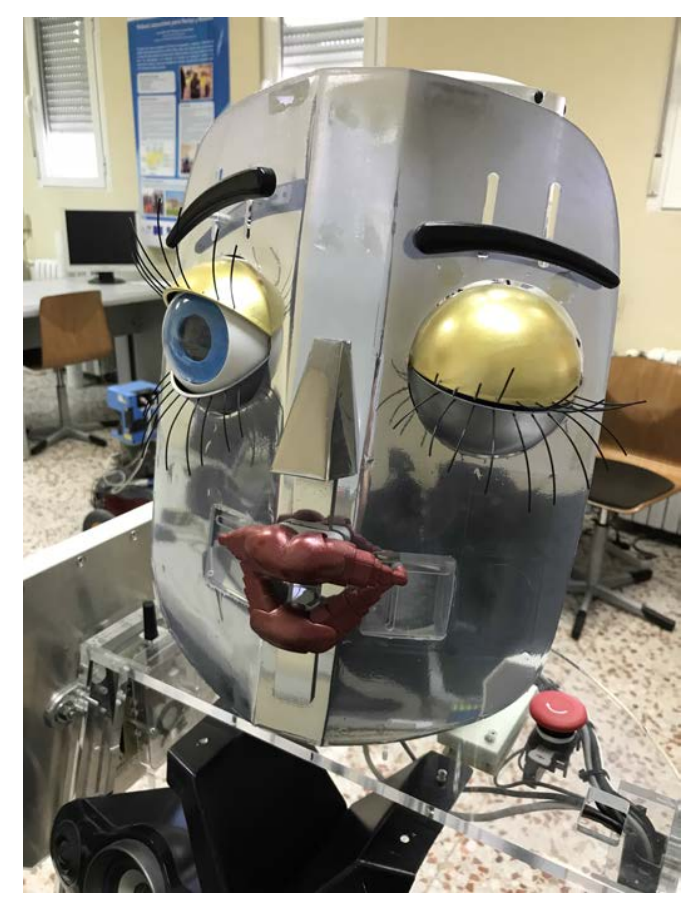

Figure 1.4: Doris blinking

The supervisory and teleoperation system is explained in Chapter 6. A web page and an android applications were developed to operate over different functions of Doris, this includes the face, the arm, the dialogs, the task planification etc. In this chapter will be detailed each step of the creation of these applications beginning with their initial conceptions, then going through the design and implementation of each application and finalizing with testing the response of each application.

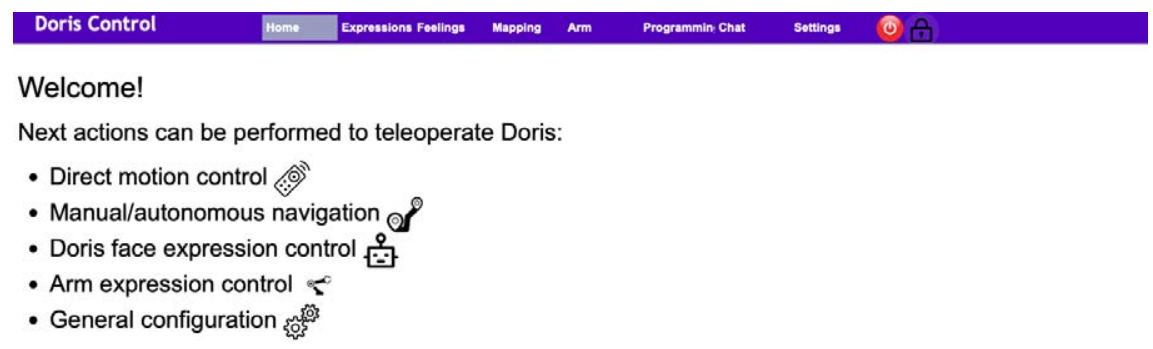

Figure 1.5: Doris webpage for teleoperation

The task planner is detailed in Chapter 7 from its conception. In the first place, grammar of the language is specified. Then, the lexical analyzer is explained, showing how to tokenize the input source. Later in the same chapter, the syntax analyzer is 
explained and how the expressions are evaluated. For expressions, a recursive descent parser is used, so an explanation of how this was implemented is explained as well. Finally, the semantic analyzer is detailed, and the results are shown, listing the script used for the demo, and links for the respective demo videos.

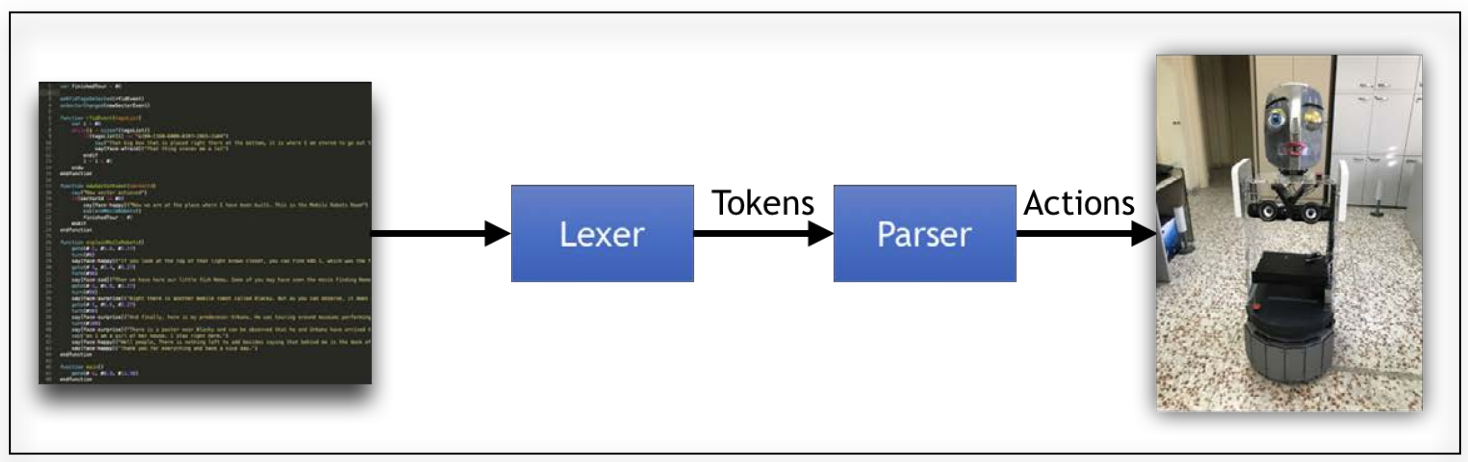

Figure 1.6: Doris interpreter for task planning

Finally, conclusions of the current state of this dissertation and future works are shown in Chapter 8 


\section{State of the art}

Several approaches have been made in order to create robots that can interact with people of all ages. Besides, special attention has been devoted to achieve widespread acceptance among the population. Thus, the variety of programmed robots ranges from waiters, domestic servants and personal assistants to guides that work in museums guiding people through the different areas and that explain sculptures and portraits, being this last one the most significant in this project, a robot named Doris and created in the Centre of Automation and Robotics.

Some other robots such as Blacky and Urbano were created in this centre as well. Blacky had the characteristic of locating itself and moving along museums using Simultaneous Localization and Mapping (SLAM), but the lack of an interface made people surround the robot trying to communicate with it, but without success. Hence, trying to improve this communication, Urbano was born, a robot that has a human like face which makes people concentrate on his visual interface.

Human face, in robotics, involves having the most common characteristics of a person's face, that is to say, eyes, eyelids, eyebrows, nose and lips. Such similarity with human faces can lead to what is called the uncanny valley, which is the degree of acceptance of people towards robots which are human like.

Both, Blacky and Urbano, where intended to work in museums as tour-guides. Hence, a set of information has to be sent to the robot so that it performs the whole package of actions needed. This involves the use of some new programming languages that were born in order to command the robot to work autonomously.

As a brief introduction to understand how Doris was programmed, control strategies such as localization and navigation methods need to be taken into account, as well as the way in which tasks are planned and some knowledge about fuzzy control and interpreted languages. 


\subsection{Interactive social robots}

MINERVA, a social robot for interaction by [1] This paper describes MINERVA (figure 2.1. an interactive tour-guide robot that was successfully deployed in a Smithsonian museum. Minerva's software is pervasively probabilistic, relying on explicit representations of uncertainty in perception and control. During 2 weeks of operation, the robot interacted with thousands of people, both in the museum and through the Web, traversing more than $44 \mathrm{~km}$ at speeds of up to $163 \mathrm{~cm} / \mathrm{sec}$ in the unmodified museum.

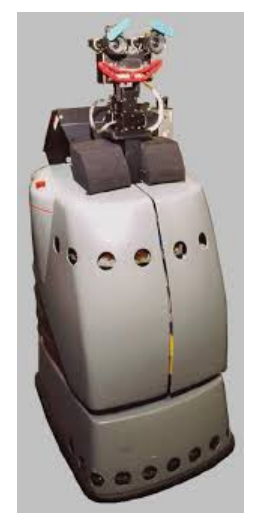

Figure 2.1: Minerva project

Other work of interactive robots working in museums is [2]. This research describes the software architecture of an autonomous, interactive tour-guide robot. It presents a modular and distributed software architecture, which integrates localization, mapping, collision avoidance, planning, and various modules concerned with user interaction and Web-based telepresence. At its heart, the software approach relies on probabilistic computation, on-line learning, and any-time algorithms. It enables robots to operate safely, reliably, and at high speeds in highly dynamic environments, and does not require any modifications of the environment to aid the robot's operation. Special emphasis is placed on the design of interactive capabilities that appeal to people's intuition. The interface provides new means for human-robot interaction with crowds of people in public places, and it also provides people all around the world with the ability to establish a virtual telepresence using the Web. The robot successfully raised the museum's attendance by more than 50\%. In addition, thousands of people all over the world controlled the robot through the Web.

[3] In this research a robot is intended to be used as a tool to offer a tour guide to the Industrial Informatics and Robotics Laboratory from Research \& Development Institute of Brasov. In this laboratory there are a lot of devices used for research and every time when new visitors or students arrive to the laboratory, an operator must explain what each device is used for. Therefore, a robot was decided to be used (namely Nao robot from Aldebaran Robotics) to welcome new visitors and perform a tour of the lab, giving explanations about the research that take place in this laboratory. Nao is very versatile 
and therefore is suitable for this purpose, being able to walk, to make gestures with its arms and hands, to speak and also to interact with peoples. The Choregraphe Software, a graphical interface is used to create simple behaviours. The robot will stay in a fixed position, at the entrance in laboratory and whenever a visitor will come, it will be ready to make a tour.

Another work by [4], presents a robot for human interaction, in particular, robot emotion, speech and facial expressions to determine if human feel comfortable in front of a machine that exhibits basic rational and intelligent behaviour.

Another research in interactive robots is [5] The aim of the robotic education is to make the students know the elements of robot and to enhance their abilities on planning applications by using the robot applications and more for experiencing the existing mechanic construction. In order to studying according to a certain curriculum, students need laboratories which they can enhance them befitting to their future settings. Practices of computer hold a place as an access and control factors. In this study, the current structure of the robotic education is accentuated, and XR3 is prepared according to experimental robot as an educational material. 2D simulation is improved which has the capacity of visualization the movement with robot synchronously and provides observing the movements of robot in advance or the opportunity of watching it visually without the need of robot's move. Rhino XR3 Robot and Mark 3 Controller which is in the constitution of Flexible Manufacturing Systems' laboratory of Gazi University Engineering and Architecture Faculty were used for this application. Delphi 6 Personal Edition Compiler was also used for the development period of software.

Another architecture can be found at [6]. This paper presents an overview of the hard and software architecture of a mobile visitor information system for the Konrad Zuse building of the School of Computer Science and Automation at the Ilmenau University of Technology. Two mobile robots serve as mobile information terminals with capabilities for generating way descriptions and guiding the visitor to the points of interest (labs, meeting rooms, offices, employees) in the building. The paper focuses on the constraints resulting from the challenging environment in this multi-floor building, as well as on the integration aspects of various skills for navigation and human-robot interaction. Besides first experience with the system, the further development is outlined as well.

The research by [7] reviews socially interactive robots: robots for which social human-robot interaction is important. Begins by discussing the context for socially interactive robots, emphasizing the relationship to other research fields and the different forms of social robots. Then, a taxonomy of design methods and system components used to build socially interactive robots is presented. Finally, the impact of these robots on humans is described and discuss open issues.

Other research performed by [8], reports on a field trial with interactive humanoid robots at a science museum where visitors are supposed to study and develop an interest 
in science. In the trial, each visitor wore an RFID tag while looking around the museum's exhibits. Information obtained from the RFID tags was used to direct the robots' interaction with the visitors. The robots, one of them is shown in figure 2.2 . autonomously interacted with visitors via gestures and utterances resembling the free play of children. In addition, they performed exhibit-guiding by moving around several exhibits and explaining the exhibits based on sensor information. The robots were highly evaluated by visitors during the two-month trial. Moreover, an experiment in the field trial to compare the detailed effects of exhibit-guiding and free-play interaction under three operating conditions was conducted. This revealed that the combination of the free-play interaction and exhibit-guiding positively affected visitors' experiences at the science museum.

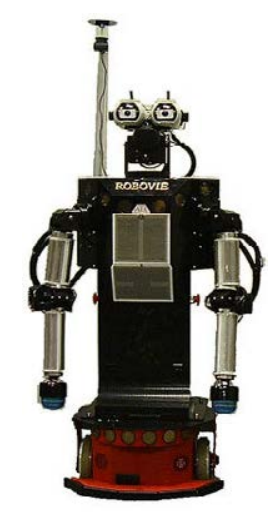

Figure 2.2: Robovie project

The research by [9] allowed to explore possible robot tasks in daily life, a guide robot for a shopping mall and conducted a field trial with it was developed. The robot was designed to interact naturally with customers and to affectively provide shopping information. It was also designed to repeatedly interact with people to build a rapport; since a shopping mall is a place people repeatedly visit, it provides the chance to explicitly design a robot for multiple interactions. For this capability, RFID tags for person identification are used. The robot was semiautonomous, partially controlled by a human operator, to cope with the difficulty of speech recognition in a real environment and to handle unexpected situations. A field trial was conducted at a shopping mall for 25 days to observe how the robot performed this task and how people interacted with it. The robot interacted with approximately 100 groups of customers each day. Customers were invited to sign up for RFID tags and those who participated answered questionnaires. The results revealed that 63 out of 235 people in fact went shopping based on the information provided by the robot. The experimental results suggest promising potential for robots working in shopping malls.

The work of [10] designed a model for social robots to report the friendship estimation that understand human social relationships. The interactive robot autonomously interacts with humans with its human-like body properties, and as a result, 
induces the humans' friendly group behaviour upon direct interaction. Based on these features, as well as inspired by a survey in psychology research on friendship, a friendship estimation model for an interactive robot was proposed. The capability provided by such a model is probably essential for interactive robots to establish social relationships with humans. The results of an experiment demonstrate that the fundamental part of the estimation model functions effectively.

One of the most notorious works is [11], which discuss about the uncanny valley (figure 2.3. This means the degree of acceptance of a machine to operate between humans. Focusing on social robots which can have any type of morphology (human, dog, cat, penguin, rabbit, etc) this machine should be morphologically acceptable and not rejected by humans. [12] is another work which completes the idea of the uncanny valley and talks about similarity with humans. The more similarity, the higher is the rejection. In order for robots to not be rejected by humans, a research performed by [13] points to a design of appearance, behavioural, interacting and learning capabilities of robots by their domestic applications, hoping that this design can achieve a humanlike appearance.

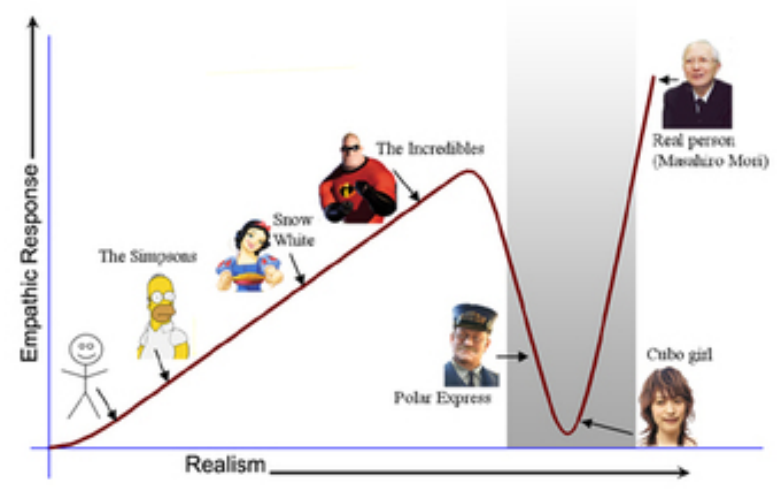

Figure 2.3: Mori's uncanny valley

Besides appearance, there are also other features like speech capabilities where the robot is expected to talk and to hear what an user says. [14] performed a research for voice recognition to control a robot by voice only. Also an emotional model is a significant feature for URBANO (figure 2.4), a social robot like the model used by [15]. 


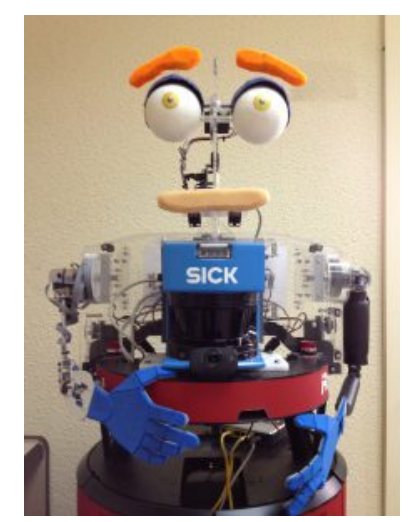

Figure 2.4: CAR's social robot URBANO

\subsection{Localization}

The presence of people, sculptures, counters, and paintings, among other factors mean that museums and trade fairs are very complex environments. Robot localization is therefore essential for any high-level task that it might perform in that kind of environment. Many studies have been aimed at achieving dependable indoor localization of robots.

The study presented by [16] presents a real-time automatic obstacle avoidance system using depth information and sonar data. The depth map is provided by Microsoft Kinect and sonar data are obtained from sonar sensors. With fusion of weighted depth information and sonar data, a set of intelligent fuzzy rules are designed to construct a safe path to avoid obstacles in indoor environment. According to the experimental results, this system has good performance while compared with the previous approaches and can work in dark environment. According to the experimental results, this system has good performance while compared with the previous approaches and can work in unfamiliar environment.

Another research performed by [17] states that autonomous localization is a primary and crucial issue in mobile robot navigation tasks. In this article, the long-distance robust localization problem of indoor mobile robots is studied and solved by a combination style of using a laser scanner and an odometer. Firstly, a point-to-line iterative closest point(PLICP) approach is adopted to match the successive environmental information collected by a laser scanner to estimate the relative pose transformation of the robot. And then the multi-sensor fusion technology based on bounded-error set-membership estimator is proposed to to use scan matching results to correct the cumulative error of the odometer periodically to achieve precious location of the robot in indoor environments. Experimental results show that the accuracy and robustness of the proposed localization system has been improved greatly with respect to the single odometer localization approach. 
Further research in sensor fusion can be found in [18]. While global navigation satellite systems (GNSS) are the state of the art for localization, in general they are unable to operate inside buildings, and there is currently no well-established solution for indoor localization. In this paper, a $3 \mathrm{D}$ mobile robot pose $(2 \mathrm{D}$ position and $1 \mathrm{D}$ orientation) estimation system for indoor applications is proposed. The system is based on the cooperative sensor fusion of radar, ultrasonic and odometry data using an extended Kalman filter (EKF). A prerequisite for the EKF is an occupancy grid map of the scenario as well as the pose of the reference radar node inside the map. The system can handle even the kidnapped-robot case as the radar provides absolute localization. A series of measurements in an office building corridor were conducted. The typical position root-mean square error (RMSE) to be less than $15 \mathrm{~cm}$ was determined.

Similar work was performed in [19], this time using an Ultra-Wideband (UWB), Indoor Positioning System (IPS) and an Inertial Navigation System (INS) achieving real-time indoor navigation and tracking of automated guided vehicles (AGVs) and mobile robots in factories and warehouses. The sensor fusion algorithm developed in this contribution consisted of delayed compensation, based on the position estimation from the IPS, and a multi-rate extended Kalman Filter that combined the delay-compensated position from the IPS and the measurements from the INS. The UWB was used to validate the sensor fusion approach, achieving a position error standard deviation of $3.7 \mathrm{~cm}$ for linear movements and 1.7 degrees for rotational movements.

The precise localization system of autonomous vehicles is one of the important techniques in the research of autonomous driving [20]. In the case of outdoor localization, the Global Positioning System (GPS) sensor is commonly used to attenuate the cumulative error of odometry data occurred from undesirable effects of slippage. However, considering GPS outages, its positioning inaccuracy would not be used as reference information for an accurate indoor localization. Therefore, in this paper, a sensor fusion system using odometry and magnetic sensor data for the precise indoor localization system is described. The odometry model is based on the Ackerman steering geometry, and directly calculated from encoder information on two rear wheels and one steering. A magnetic sensor is developed to measure magnetic field produced from circular-type magnets located in the ground. Considering the sensor data fusion, Unscented Kalman Filter (UKF) is utilized so that the mean and covariance of the nonlinear system function can be estimated by the Unscented Transformation (UT) with sigma points. The simulation results are presented that demonstrate the validity of the UKF based localization system.

The research by [21] proposes a geometric feature-based method to solve the Simultaneous Localization and Mapping (SLAM) problem in an unknown structured environment using a short range and low Field of View (FoV) measurement unit such as Kinect sensor. A RANdom SAmple Consensus (RANSAC) based algorithm is used for feature detection, and a grid-based point cloud segmentation method has been introduced to improve the multiple feature point-detection in a 2D depth frame. A fast SLAM 
algorithm is used to estimate the robot posterior and the map of the environment. This approach builds the individual maps for each particle using geometric features that are extracted from a 2D slice of a 3D depth image. Each map contains individual Extended Kalman Filters (EKFs) for each and every feature-point. This method reduces the uncertainty of the robot pose in the prediction step and it improves the pose accuracy when more geometric feature-points are available. The proposed feature-based approach gives better localization and compact map representation in structured environments when distinct features are available. The importance weighting and the comparison of features with the on-line map are performed according to the maximum likelihood criterion. In order to reduce the particle depletion, the map is updated only when a new Odometry measurement and new range measurements are available. The experiments are carried out using the recorded data with a non-holonomic mobile robot equipped with a Kinect sensor in a small scale indoor structured environment. For comparison, the grid based SLAM result is also presented for the same data set.

Mobile robot localization with different sensors and algorithms is a widely studied problem [22], and there have been many approaches proposed, with considerable degrees of success. However, every sensor and algorithm has limitations, due to which it is believed that no single localization algorithm can be perfect, or universally applicable to all situations. Laser rangefinders are commonly used for localization, and state-of-the-art algorithms are capable of achieving sub-centimeter accuracy in environments with features observable by laser rangefinders. Unfortunately, in large scale environments, there are bound to be areas devoid of features visible by a laser rangefinder, like open atria or corridors with glass walls. In such situations, the error in localization estimates using laser rangefinders could grow in an unbounded manner. Localization algorithms that use depth cameras, like the Microsoft Kinect sensor, have similar characteristics. WiFi signal strength based algorithms, on the other hand, are applicable anywhere there is dense WiFi coverage, and have bounded errors. Although the minimum error of WiFi based localization may be greater than that of laser rangefinder or depth camera based localization, the maximum error of WiFi based localization is bounded and less than that of the other algorithms.

The research [23] presents a novel approach to mobile robot localization using sonar sensors. This approach is based on the use of particle filters. Each particle is augmented with local environment information which is updated during the mission execution. An experimental characterization of the sonar sensors used is provided in the paper. A probabilistic measurement model that takes into account the sonar uncertainties is defined according to the experimental characterization. The experimental results quantitatively evaluate the presented approach and provide a comparison with other localization strategies based on both the sonar and the laser. Some qualitative results are also provided for visual inspection.

Studies in external environments also perform a sensor fusion like in [24]. This paper describes a robust localization method for an outdoor robot that gives tours of the 
Rice University campus. The robot fuses odometry and GPS data using extended Kalman filtering. A technique for handling two types of nonstationarity in GPS data quality is proposed and experimentally tested: abrupt changes in GPS position readings caused by sudden obstructions to line of sight access to satellites, and more gradual changes caused by disparities in atmospheric conditions. Measurement error covariance matrices indexed by number of visible satellites and switch them into the localization computation automatically are constructed. The matrices are built by sampling GPS data repeatedly along the route and are updated continuously to handle drift in GPS data quality. The approach performs better than extended Kalman filters that use only a single error covariance matrix is demonstrated. With a GPS receiver that delivers 1 meter accuracy, it has been possible to localize at $40 \mathrm{~cm}$ through a challenging route in the Engineering Quadrangle of Rice University.

Studies by the Intelligent Control Group of the Centre of Automation and Robotics of the UPM-CSIC were performed on sensor fusion, such as [25]Among the solutions to the simultaneous localization and mapping (SLAM) problem with probabilistic techniques, the extended Kalman filter (EKF) is a very common approach. There are several approaches to deal with its computational cost, usually based on an adequate selection of features to be updated in real time, while the whole map update is delayed or processed in a background task, allowing one to map larger environments or to carry out multirobot experiments. Although these solutions are theoretically sound, there is a great lack of real experiments in large indoor environments due to several previously unknown problems derived from the geometric model of the map features and the inconsistency of the SLAM-EKF algorithm. For the first time, these problems are described and solved, and the implementation of the algorithms and solutions presented in this paper achieve excellent results in experiments in different real large indoor environments.

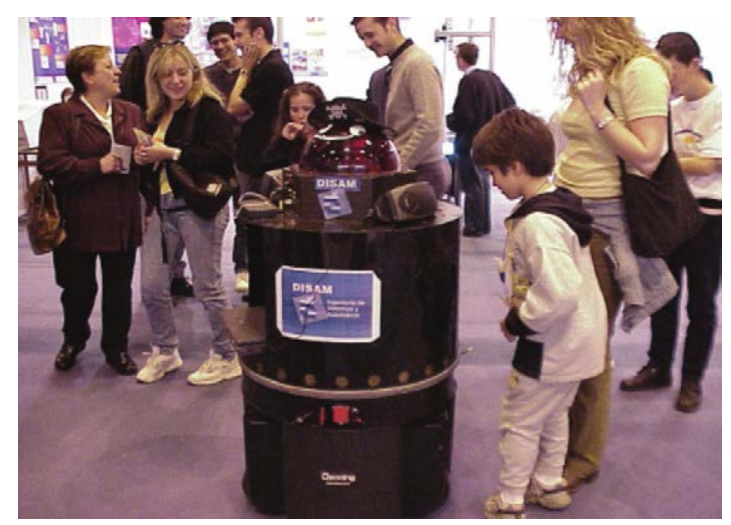

Figure 2.5: CAR's robot BLACKY

The same author proposed an efficient geometric approach for Simultaneous Localization And Mapping (SLAM) based on an extended Kalman Filter in [26] This paper presents an efficient geometric approach to the Simultaneous Localization and Mapping problem based on an Extended Kalman Filter. The map representation and 
building process is formulated, fully implemented and successfully experimented in different indoor environments with different robots. The use of orthogonal shape constraints is proposed to deal with the inconsistency of the estimation. Built maps are successfully used for the navigation of two different service robots: an interactive tour guide robot, and an assistive walking aid for the frail elderly.

Some years later, the FastSlam, an upgrade to the SLAM problem, was presented in [27] The process of building a map with a mobile robot is known as the Simultaneous Localization and Mapping (SLAM) problem, and is considered essential for achieving true autonomy. The best existing solutions to the SLAM problem are based on probabilistic techniques, mainly derived from the basic Bayes Filter. A recent approach is the use of Rao-Blackwellized particle filters. The FastSLAM solution factorizes the Bayes SLAM posterior using a particle filter to estimate over the possible paths of the robot and several independent Kalman Filters attached to each particle to estimate the location of landmarks conditioned to the robot path. Although there are several successful implementations of this idea, there is a lack of applications to indoor environments where the most common feature is the line segment corresponding to straight walls. This paper presents a novel factorization, which is the dual of the existing FastSLAM one, that decouples the SLAM into a map estimation and a localization problem, using a particle filter to estimate over maps and a Kalman Filter attached to each particle to estimate the robot pose conditioned to the given map. This approach was implemented and tested, analyzing and comparing the solution provided with the FastSLAM one, and successfully building feature based maps of indoor environments.

In the same year, [28] describes a simultaneous localization and mapping (SLAM) algorithm for use in unstructured environments that is effective regardless of the geometric complexity of the environment. Features are described using B-splines as modeling tool, and the set of control points defining their shape is used to form a complete and compact description of the environment, thus making it feasible to use an extended Kalman-filter (EKF) based SLAM algorithm. This method is the first known EKF-SLAM implementation capable of describing general free-form features in a parametric manner. Efficient strategies for computing the relevant Jacobians, perform data association, initialization, and map enlargement are presented. The algorithms are evaluated for accuracy and consistency using computer simulations, and for effectiveness using experimental data gathered from different real environments.

Other SLAM research was performed in [29], by introducing a priori knowledge about the latent structure of the environment in simultaneous localization and mapping (SLAM), can improve the quality and consistency results of its solutions. In this paper a general framework for the detection, evaluation, incorporation and removal of structure constraints into a feature-based graph formulation of SLAM is described and analyzed. The inclusion of different kinds and levels of features in a hierarchical manner allows the system to easily discover new structure and why it makes more sense than other possible representations are shown. The main algorithm in this framework follows an expectation 
maximization approach to iteratively infer the most probable structure and the most probable map. Experimental results show how this approach is suitable for the integration of a large variety of constraints and how the method can produce nice and consistent maps in regular environments.

Localization based on Wireless Networks is attributed high importance in recent research, among which the most common system is Radio Frequency Identification (RFID). Different approaches have been developed, such as [30] that introduces a positioning algorithm for RFID tags using two mobile readers that process passive or active tags with randomly distributed known locations. The location was estimated using multilateration with the landmarks and a probabilistic RFID map-based technique with Kalman Filtering.

Another study was developed by [31] Radio frequency identification (RFID) is poised for growth as businesses and governments explore applications implementing RFID. The RFID technology will continue to evolve to meet new demands for human and target location and tracking. In particular, there are increasing needs to locate and track multiple RFID-tagged items that are closely spaced. As a result, localization and tracking techniques with higher accuracy yet low implementation complexity are required. This paper examines the applicability of direction-of-arrival (DOA) estimation methods to the localization and tracking problems of passive RFID tags. Different scenarios of stationary and moving targets are considered. It is shown through performance analysis and simulation results that simple DOA estimation methods can be used to provide satisfactory localization performance.

A subsequent RFID positioning system was implemented by [32], based on an object carrying an RFID reader module that reads low-cost passive tags installed next to the object path and that uses a Kalman Filter, the measurements of which are the backscattered signal power propagated from the nearest RFID tag and a tag-path position database. The algorithm is used to produce an estimate of the location of the reader, neglecting tag-reader angle-path loss, where it is compensated by the signal strength information measurement that is received.

The study presented by [33] addresses a radio-frequency identification (RFID)-based mobile robot localization which adopts RFID tags distributed in a space. Existing standalone RFID systems for mobile robot localization are hampered by many uncertainties. Therefore, a novel algorithm that improves the localization by fusing an RFID system with an ultrasonic sensor system is proposed. The system partially removes the uncertainties of RFID systems by using distance data obtained from ultrasonic sensors. A global position estimation (GPE) process using an RFID system and a local environment cognition (LEC) process using ultrasonic sensors is defined. Then, a hierarchical localization algorithm is proposed to estimate the position of the mobile robot using both GPE and LEC. Finally, the utility of the proposed algorithm is demonstrated through experiments. 
In an attempt to find an accurate, environmentally robust, and fast process for RFID distance estimations, a method was developed called RSSI Informed Phase. This method uses both phase and RSSI measurements to estimate the distance, and potentially location, of a moving RFID tag. RSSI is initially used to find an approximate reader to tag separation distance. This distance value is applied to find an approximate slope of the phase angle vs. frequency curve. The estimated slope can inform the phase distance calculation, meaning fewer reads are required to find the actual phase angle vs. frequency slope. The reduction in the number of necessary reads accelerates the localization process and makes this method more robust for dynamic environments [34].

More recent research has been performed in [35] referencing intelligent transportation system applications that require accurate, reliable, and continuous vehicle positioning. How to achieve such positioning performance in extended GPS-denied environments such as tunnels is the main challenge for land vehicles. This paper proposes a hybrid multi-sensor fusion strategy for vehicle positioning in tunnels. First, the preliminary positioning algorithm is developed. The Radio Frequency Identification (RFID) technology is introduced to achieve preliminary positioning in the tunnel. The received signal strength (RSS) is used as an indicator to calculate the distances between the RFID tags and reader, and then a Least Mean Square (LMS) federated filter is designed to provide the preliminary position information for subsequent global fusion. Further, to improve the positioning performance in the tunnel, an interactive multiple model (IMM)-based global fusion algorithm is developed to fuse the data from preliminary positioning results and low-cost in-vehicle sensors, such as electronic compasses and wheel speed sensors. In the actual implementation of IMM, the strong tracking extended Kalman filter (STEKF) algorithm is designed to replace the conventional extended Kalman filter (EKF) to achieve model individual filtering. Finally, the proposed strategy is evaluated through experiments. The results validate the feasibility and effectiveness of the proposed strategy.

In [36], RFID (Radio Frequency Identification) offers a way to identify objects without any contact. However, positioning accuracy is limited since RFID neither provides distance nor bearing information about the tag. This paper proposes a new and innovative approach for the localization of moving object using a particle filter by incorporating RFID phase and laser-based clustering from $2 \mathrm{~d}$ laser range data. First of all, the phase-based velocity of the moving object based on RFID phase difference is calculated. Meanwhile, laser range data is separated into different clusters, and compute the distance-based velocity and moving direction of these clusters. Then the similarity between two velocities, and select $\mathrm{K}$ clusters having the best similarity score are computed and analyzed. A prediction of the particles according to the velocity and moving direction of laser clusters was performed. Finally, the weights of the particles were updated based on K clusters and achieve the localization of moving objects. The feasibility of this approach is validated on a Scitos G5 service robot and the results prove that we have successfully achieved a localization accuracy up to $0.25 \mathrm{~m}$. 


\subsection{Control strategies}

The paper [37] focuses on the use of spherical cameras for autonomous robot navigation tasks. In this paper, the spherical image navigation as an image classification problem is proposed, which significantly simplifies the orientation estimation and path prediction procedure and accelerates the navigation process. More specifically, an end-to-end convolutional network is trained on the spherical image dataset with novel orientation categories labels. This trained network can give precise predictions on potential path directions with single spherical images. Experimental results on the Spherical-Navi dataset demonstrate that the proposed approach outperforms the comparing methods in realistic applications.

Another paper [38] tests if it is possible to train neural networks to control the robot to reach the target location in urban dynamic environments. The results show that neural controller with separated hidden neurons has a fast response to sensory input. The performance of evolved neural controllers is also tested in real robot navigation. In addition to the neural network based navigation, the robot has also to switch between other navigation algorithms to reach the target location in the university campus.

[39] In this paper, a fuzzy logic system is designed and an obstacle avoidance algorithm for a path planning in unknown environment for a mobile robot is proposed. A new rule table is proposed, that is induced from the consideration of the distance to obstacles and the angle between the robot and the goal. Some simulation results show that the proposed method generates a good path with avoiding obstacles and has faster travelling time.

In [40] a neural network learns to avoid obstacles as the robot moves around without supervision in a cluttered environment. The neural network does not require any knowledge about the quality or configuration of the sensors. In this article we report results using our neural network with the real mobile robot Khepera.

An online navigation technique for a wheeled mobile robot (WMR) in an unknown dynamic environment using fuzzy logic techniques is investigated in [41]. In this paper, the aim is to use the robot in application in a warehouse.

The research [42] describes the design and the implementation of a trajectory tracking controller using fuzzy logic for mobile robot to navigate in indoor environments. Most of the previous works used two independent controllers for navigation and avoiding obstacles. The main contribution of the paper can be summarized in the fact that we use only one fuzzy controller for navigation and obstacle 
avoidance. The used mobile robot is equipped with DC motor, nine infrared range (IR) sensors to measure the distance to obstacles, and two optical encoders to provide the actual position and speeds. To evaluate the performances of the intelligent navigation algorithms, different trajectories are used and simulated using MATLAB software and SIMIAM navigation platform. Simulation results show the performances of the intelligent navigation algorithms in terms of simulation times and travelled path.

Dealing with trajectory stabilization of a computer simulated model car via fuzzy control is proposed in [43]. Stability conditions of fuzzy systems are given in accordance with the definition of stability in the sense of Lyapunov. First, a model of the car is simulated by computer, whose dynamics is nonlinear, by T-S (Takagi and Sugeno) fuzzy model. Fuzzy control rules, which guarantee stability of the control system under a condition, are derived from the approximated fuzzy model. The simulation results show that the fuzzy control rules effectively realize trajectory stabilization of the model car along a given reference trajectory from all initial positions under a condition and the dynamics of the approximated fuzzy model agrees well with that of the model car.

The paper [44] is concerned with the problem of reactive navigation for a mobile robot in an unknown clustered environment. A reactive navigation is defined as a mapping between sensory data and commands. Building a reactive navigation system means providing such a mapping. It can come from a family of predefined functions (like potential fields methods) or it can be built using 'universal' approximators (like neural networks). In this paper, will be consider another 'universal' approximator: fuzzy logic. it will be explained how to choose the rules using a behaviour decomposition approach. It is possible to build a controller working quite well but the classical problems are still there: oscillations and local minima. Finally, we will conclude that learning is necessary for a robust navigation system and fuzzy logic is an easy way to put some initial knowledge in the system to avoid learning from zero.

Navigation techniques for several mobile robots as many as one thousand robots using fuzzy logic are investigated in a totally unknown environment. Fuzzy logic controllers (FLC) using different membership functions are developed and used to navigate mobile robots. First a fuzzy controller has been used with four types of input members, two types of output members and three parameters each. Next two types of fuzzy controllers have been developed having same input members and output members with five parameters each. Each robot has an array of ultrasonic sensors for measuring the distances of obstacles around it and an infrared sensor for detecting the bearing of the target. These techniques have been demonstrated in various exercises, which depicts that the robots are able to avoid obstacles as well as negotiate the dead ends and reach the targets efficiently. Amongst the techniques developed, FLC having Gaussian membership function is found to be most efficient for mobile robots navigation [45].

The research [46] presents a new methodology for the avoidance of one or more obstacles, and for the navigation of a differential-drive mobile robot. The approach is 
based on fuzzy logic with virtual fuzzy magnets, and represents a reactive controller for navigation through an unknown environment to the ultimate target. Relative parameters of the obstacle in the robot's way are determined at the preprocessing stage and the algorithm is therefore applicable to obstacles of different sizes. The algorithm, designed to avoid a single stationary obstacle, was generalized and successfully applied in a multiple-obstacle navigation scenario. The efficiency of the algorithm is illustrated by computer simulations using the kinematic model of a mobile robot.

As shown in previous works, most of control techniques are based in fuzzy logic, for that reason, basic concepts of this area of expertise will be explained for future developments.

\subsubsection{Fuzzy logic}

Over the past several years, fuzzy control has emerged as one of the most active areas of research in control engineering. The pioneering research of Mamdani and his colleagues [47] [48] [49] on fuzzy control was motivated by Zadeh's seminal papers on the linguistic approach and system analysis based on theory of fuzzy sets [50]. Applications of fuzzy control in such diverse areas have pointed the way for the utilization of fuzzy control in the context of ill-defined processes that can be controlled by a skilled human operator without the knowledge of their underlying dynamics.

Fuzzy logic provides a computationally-oriented system of concepts and techniques for dealing with modes of reasoning which are approximate rather than exact. Approximate reasoning is essential for simulation human decision-making behavior in an environment of uncertainty and imprecision can be studied in a formal way.

\section{Fuzzy sets}

The concept of a fuzzy set can be viewed as an extension of an ordinary set [50] [51]. In a fuzzy set, an element can be a member of the set with a degree of membership varying between 0 and 1 . Thus a fuzzy set A with a domain $\mathbf{X}=x$ is a set of ordered pairs.

$$
\mathbf{A}=\left\{\left(x ; \mu_{A}(x)\right) \forall x \in X\right\}
$$

where $\mu_{A}$ is the characteristic membership function of A defined as:

$$
\mu_{A}: U \rightarrow[0,1]
$$

Which is the compatibility degree of a predicate, in other words, it is the possibility degree that the predicate is true, and it is not the probability. 


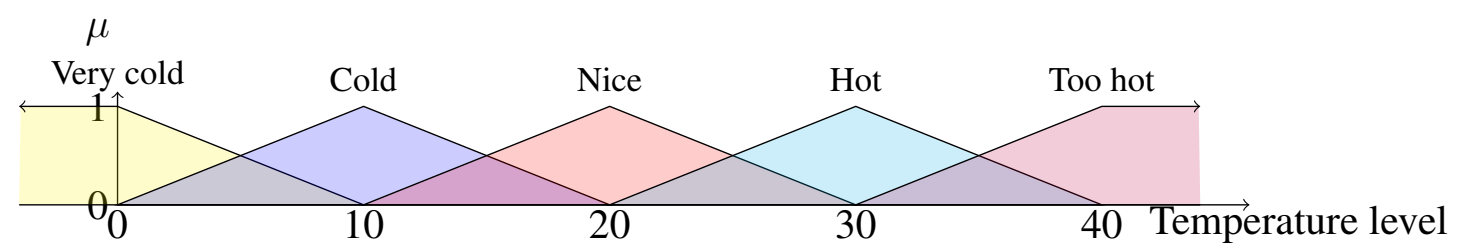

Figure 2.6: Representation of a fuzzy set used to define environment temperature

\section{Linguistic Variable}

A linguistic variable can be regarded either as a variable whose values are fuzzy members (fuzzy sets which are normal and convex) or as a variable whose values are defined in linguistic terms. To illustrate, if temperature from figure 2.6 is interpreted as a linguistic variable, then the linguistic values of temperatures might be Very cold, Cold, Nice, Hot, Too hot.

In a particular context, very cold may be interpreted as a temperature below about 0 Celsius degrees, Cold as temperature about 10 Celsius degrees, Nice as a temperature close to 20 Celsius degrees, Hot as a temperature close to 30 Celsius degrees and Very hot as a temperature close to 40 Celsius degrees.

\subsubsection{Fuzzy logic system}

Most of the fuzzy logic literature deals with mappings from fuzzy sets into fuzzy sets. In many applications of fuzzy logic to engineering problems, the main interest lies in mapping from numbers into numbers, and not sets into sets. Consequently, the main problem is more difficult than the usual fuzzy logic problem. Therefor, a frontend fuzzifier and a rear-end defuzzifier need to be added compared to the usual fuzzy logic model. The result is a fuzzy logic system (FLS).

Figure 2.7 shows the basic configuration of a fuzzy logic system. The four principal components of a fuzzy logic system are a fuzzifier, rule base, inference engine, and defuzzifier. The fuzzifier performs a scale mapping which translates the range of values of input variables into corresponding universes of discourse, as well function of fuzzification which converts input data into suitable linguistic values. The fuzzy rule base comprises of a collection of fuzzy IF-THEN rules which describes the relationship between input states and output states. The decision making logic, the inference engine of the systems, emulates human decision-making behavior based on the principles of approximate reasoning. The defuzzifier takes a fuzzy output from inference engine and determines a crisp output variable. Moreover, the defuzzifier performs a scale mapping, which converts the range of values of output variables into the corresponding application domain. 


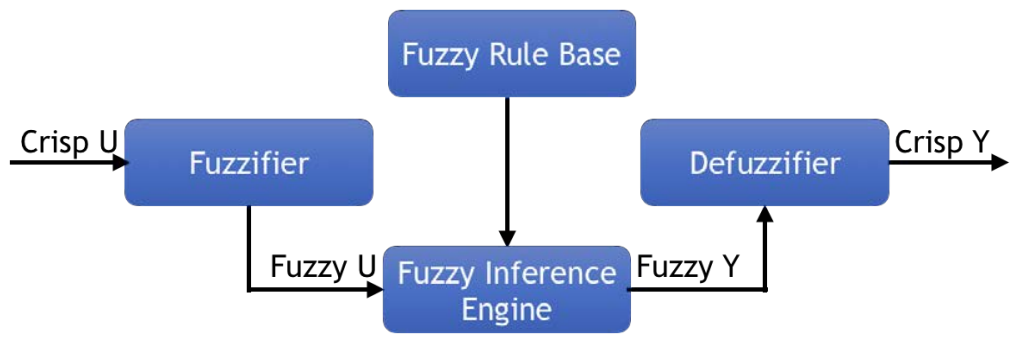

Figure 2.7: Basic configuration of a fuzzy logic system

\section{Fuzzy rule base}

The fuzzy rule base consist of a collection of fuzzy IF-THEN rules:

$$
R^{(l)}: \text { if } x_{1} \text { is } M_{1}^{l} \text { and } \ldots \text { and } x_{n} \text { is } M_{n}^{l} \text { then } y \text { is } G^{l}
$$

Where $x=\left(x_{1}, \ldots, x_{n}\right)^{T} \in U$ and $y \in Y$ are the real inputs and the real output of the fuzzy logic system, respectively, $M_{i}^{l}$ and $M^{l}$ are labels of the fuzzy sets in $X_{i}$, $Y$ respectively. Each fuzzy rule IF-THEN, defines a fuzzy implication $M_{1}^{l} \times \ldots \times M_{n}^{l}$, which is a fuzzy set defined in the product space $U \times Y$. Based on generalizations of implications in multivalue logic, many fuzzy implication rules have been proposed in the fuzzy logic literature.

\section{Fuzzy Inference Engine}

The fuzzy inference engine performs a mapping from fuzzy sets in $U$ to fuzzy sets in $Y$, based upon the fuzzy IF-THEN rules in the fuzzy rule base and the compositional rule of inference. Let $A_{x}$ be an arbitrary fuzzy set in $U$; then each $R^{(l)}$ determines a fuzzy set $A_{x} \circ R^{(l)}$, in Y based on the following sup-star compositional rule of inference:

$$
\sup _{x \in U}\left[\mu_{A_{x}}(x) * \mu_{F_{1}^{l} \times \ldots \times F_{n}^{l} \rightarrow G^{l}}(x, y)\right]
$$

where $\mu_{F_{1}^{l} \times \ldots \times F_{n}^{l} \rightarrow G^{l}}(x, y)$ is determined by the fuzzy implication rules. The final fuzzy set, $A_{x} \circ\left(R^{1}, \ldots, R^{M}\right)$, determined by all the $\mathrm{M}$ rules in the fuzzy rule base is obtained using fuzzy disjunction:

$$
\mu_{A_{x} \circ\left(R^{1}, \ldots, R^{M}\right)}(y)=\mu_{A_{x} \circ\left(R^{1}\right)}(y) \dot{+} \ldots \dot{+} \mu_{A_{x} \circ\left(R^{M}\right)}(y)
$$

Where $*$ denotes the t-norm and the $\dot{+}$ denotes a $\mathrm{t}$-conorm.

\section{Fuzzifier}

The fuzzifier maps a crisp point $x=\left(x_{1}, \ldots, x_{n}\right)^{T}$ into a fuzzy set $A_{x}$ in $U$. There are (at least) two possible choices of this mapping: 
- $A_{x}$ is a fuzzy singleton with support $x$; i.e., $\mu_{A_{x}}\left(x^{\prime}\right)=1$ for $x^{\prime}=x$ and $\mu_{A_{x}}\left(x^{\prime}\right)=$ 0 for all the other $x \in U$ with $x^{\prime} \neq x$.

- $\mu_{A_{x}}(x)=1$ and $\mu_{A_{x}}\left(x^{\prime}\right)$ decreases from 1 as $x^{\prime}$ moves away from $x$.

In the literature, it seems that only the singleton fuzzifier has been used. However, the nonsingleton fuzzifier may be useful for inputs which are corrupted by noise.

\section{Defuzzifier}

The defuzzifier maps fuzzy sets in $\mathrm{Y}$ to a crisp set in $\mathrm{Y}$. the most common choice of this mapping is the center-average (or centroid) defuzzifier, defined as:

$$
y=\frac{\sum_{i=1}^{M}\left(y^{i}\right)\left(\mu_{A_{x} \circ R^{(l)}}\left(y^{i}\right)\right)}{\sum_{i=1}^{M}\left(\mu_{A_{x} \circ R^{(l)}}\left(y^{i}\right)\right)}
$$

Where is $y^{i}$ the point in $Y$ which $\mu_{G^{i}}(y)$ achieves its maximum value and $\mu_{A_{x} \circ R^{(l)}}$ is given by sup-star compositional rule of inference.

\subsection{Path planning}

The research [52], describes the problem of goals nonreachable with obstacles nearby when using potential field methods for mobile robot path planning. Then, new repulsive potential functions are presented by taking the relative distance between the robot and the goal into consideration, which ensures that the goal position is the global minimum of the total potential.

An approach to robot path planning that consists of incrementally building a graph connecting the local minima of a potential field defined in the robot's configuration space and concurrently searching this graph until a goal configuration is attained is proposed [53]. Unlike the so-called global path planning methods, this approach does not require an expensive computation step before the search for a path can actually start, and it searches a graph that is usually much smaller than the graph searched by the so-called local methods. A collection of effective techniques to implement this approach is described. They are based on the use of multiscale pyramids of bitmap arrays for representing both the robot's workspace and configuration space. This distributed representation makes it possible to construct potential fields numerically, rather than analytically. A path planner based on these techniques has been implemented. Experiments with this planner show that it is both very fast and capable of handling many degrees of freedom.

An approach to robotic path planning [54], which allows optimization of useful performance indices in the presence of obstacles, is given. The main idea is to express 
obstacle avoidance in terms of the distances between potentially colliding parts. Mathematical properties of the distance functions are studied and it is seen that various types of derivatives of the distance functions are easily characterized. The results lead to the formulation of path planning problems as problems in optimal control and suggest numerical procedures for their solution. A simple numerical example involving a three-degree-of-freedom Cartesian manipulator is described.

The problem of automatic collision-free path planning is central to mobile robot applications [55]. An approach to automatic path planning based on a quadtree representation is presented. Hierarchical path-searching methods are introduced, which make use of this multiresolution representation, to speed up the path planning process considerably. The applicability of this approach to mobile robot path planning is discussed.

A method is presented for planning a path in the presence of moving obstacles [56]. Given a set of polygonal moving obstacles, the authors focus on generating a path for a mobile robot that navigates in the two-dimensional plane. Their methodology is to include time as one of the dimensions of the model world. This enables the authors to regard the moving obstacles as being stationary in the extended world. For a solution to be feasible, the robot must not collide with any other moving obstacles, and, also, it must navigate without exceeding the predetermined range of velocity, acceleration, and centrifugal force. The authors investigate an appropriate model to represent the extend world for the path-planning task, and give a time-optimal solution using this model

A knowledge based genetic algorithm (GA) for path planning of a mobile robot is proposed [57], which uses problem-specific genetic algorithms for robot path planning instead of the standard GAs. The proposed knowledge based genetic algorithm incorporates the domain knowledge into its specialized operators, where some also combine a local search technique. The proposed genetic algorithm also features a unique and simple path representation and a simple but effective evaluation method. The knowledge based genetic algorithm is capable of finding an optimal or near-optimal robot path in both complex static and dynamic environments. The effectiveness and efficiency of the proposed genetic algorithm is demonstrated by simulation studies. The irreplaceable role of the specialized genetic operators in the proposed GA for solving robot path planning problem is demonstrated by a comparison study.

Determination of a collision free path for a robot between start and goal positions through obstacles cluttered in a workspace is central to the design of an autonomous robot path planning. [58] presents an overview of autonomous mobile robot path planning focusing on algorithms that produce an optimal path for a robot to navigate in an environment. To complete the navigation task, the algorithms will read the map of the environment or workspace and subsequently attempts to create free paths for the robot to traverse in the workspace without colliding with objects and obstacles. Appropriate or correct and suitable algorithms will fulfil its function fast enough, that is, to find an 
optimal path for the robot to traverse in, even if there are a large number of obstacles cluttered in a complex environment. To achieve this, various approaches in the design of algorithms used to develop an ideal path planning system for autonomous mobile robots have been proposed by many researchers. Simulation and experimental results from previous research shows that algorithms play an important role to produce an optimal path (short, smooth and robust) for autonomous robot navigation and simultaneously it prove that appropriate algorithms can run fast enough to be used practically without time-consuming problem. This paper presents an overview and discusses the strength and weakness of path planning algorithms developed and used by previous and current researchers.

In a research performed in [59], the authors introduce a novel RRT extend function for wheeled mobile robots. The approach computes closed-loop forward simulations based on the kinematic model of the robot and enables the planner to efficiently generate smooth and feasible paths that connect any pairs of states. The authors extend the control law of an existing discontinuous state feedback controller to make it usable as an RRT extend function and prove that all relevant stability properties are retained. We study the properties of the new approach as extender for RRT and RRT* and compare it systematically to a spline-based approach and a large and small set of motion primitives. The results show that our approach generally produces smoother paths to the goal in less time with smaller trees. For RRT*, the approach produces also the shortest paths and achieves the lowest cost solutions when given more planning time.

Robots that work in a proper formation show several advantages compared to a single complex robot, such as a reduced cost, robustness, efficiency and improved performance [60] . Existing researches focused on the method of keeping the formation shape during the motion, but usually neglect collision constraints or assume a simplified model of obstacles. This paper investigates the path planning of forming a target robot formation in a clutter environment containing unknown obstacles. The contribution lies in proposing an efficient path planner for the multiple mobile robots to achieve their goals through the clutter environment and developing a dynamic priority strategy for cooperation of robots in forming the target formation. A multirobot system is set up to verify the proposed method of robot path planning. Simulations and experiments results demonstrate that the proposed method can successfully address the collision avoidance problem as well as the formation forming problem.

A research proposes a novel Rapidly exploring random tree algorithm on rough terrains (RRT-RT) for the purpose of outdoor mobile robot navigation. Differently from other RRTs adopted for rough terrains where finding a nearest neighbuor from a new random state within the tree is based on Euclidian distance, the proposed algorithm uses a roughness based metric. The metric is defined by the help of the roughness based navigation function, RbNF, that represents an estimate of the roughness-to-go value from each terrain location to the goal position. Simulation study shows that the RRT-RT provides an effective way to explore more promising terrain regions in order to decrease 
the total roughness along the resulting path [61].

An approach to robot perception and world modeling that uses a probabilistic tesselated representation of spatial information called the occupancy grid is reviewed [62]. The occupancy grid is a multidimensional random field that maintains stochastic estimates of the occupancy state of the cells in a spatial lattice. To construct a sensor-derived map of the robot's world, the cell state estimates are obtained by interpreting the incoming range readings using probabilistic sensor models. Bayesian estimation procedures allow the incremental updating of the occupancy grid, using readings taken from several sensors over multiple points of view. The use of occupancy grids from mapping and for navigation is examined. Operations on occupancy grids and extensions of the occupancy grid framework are briefly considered.

Discrete Bayesian models have been used to model uncertainty for mobile-robot navigation, but the question of how actions should be chosen remains largely unexplored. This paper presents the optimal solution to the problem, formulated as a partially observable Markov decision process. Since solving for the optimal control policy is intractable, in general, it goes on to explore a variety of heuristic control strategies. The control strategies are compared experimentally, both in simulation and in runs on a robot [63].

Research on mobile robot navigation has produced two major paradigms for mapping indoor environments: grid-based and topological [64]. While grid-based methods produce accurate metric maps, their complexity often prohibits efficient planning and problem solving in large-scale indoor environments. Topological maps, on the other hand, can be used much more efficiently, yet accurate and consistent topological maps are considerably difficult to learn in large-scale environments. This paper describes an approach that integrates both paradigms: grid-based and topological. Grid-based maps are learned using artificial neural networks and Bayesian integration. Topological maps are generated on top of the grid-based maps, by partitioning the latter into coherent regions. By combining both paradigms' grid-based and topological, the approach presented here gains the best of both worlds: accuracy/consistency and efficiency. The pa- per gives results for autonomously operating a mobile robot equipped with sonar sensors in populated multi-room environments.

\subsection{Programming languages}

Concerning task planning, several research has been done to implement the sequential execution of tasks. Different types of programming languages can be found in [65]. Great attentions have been put on the programming technologies to construct robot software in both academic research and industry due to the increasingly wide applications of robots in various areas, and potential challenges resulting from the 
complexity of robot software. In the past years, diverse programming technologies and languages have been designed to support the development of robot software. However, with robot applications and requirements change, to develop robot software remains a great challenge, especially when robots are widely used in open environment and expected to provide better and friendly services for human beings. This paper aims at analyzing the technical requirements for designing robot programming language, presenting the roadmap of the robot programming language and discussing its trends and potential challenges.

Here are mentioned some languages as ROBOL/0 [66] The behaviour of an autonomous mobile robot must be executed based on the sensor information. The sensor-based behaviuor cannot be represented by a simple sequence of motions. Action mode representation and ROBOL/0 language are a powerful method to represent such sensor-based behaviour. Autonomous navigation with the obstacle avoidance function is designed and represented using action modes. To show the effectiveness of the method, it is programmed in ROBOL/0 and executed on a real robot..

Other programming language mentioned is [67] The flexibility of a robot system comes from its ability to be programmed. How the robot is programmed is a main concern of all robot users. A good mechanical arm can be underutilized if it is too difficult to program. The introduction of the Universal Robot Controller (URC) has made the possibility of a standard, easy to use, robot programming language a reality. The URC is an open-architecture, PC?based robot controller. It will work with virtually any robot and gives the user increased flexibility and capabilities over the standard OEM controllers. The URC uses Windows NT as its operating system. The URC is the ideal platform for a universal robot programming language, RobotScript. It allows one robot language to run all robots in a factory.

Other work performed by [68] where the research proposes a new logic programming language called GOLOG which maintains a representation of the dynamic world. This language was programmed in PROLOG and appears well suited for applications in high level control of robots and industrial processes.

A related work can also be found in [69] which is an extension of GOLOG including concurrency. This concurrency gives facilities for prioritizing the execution of concurrent processes, interrupting the execution when certain conditions become true and dealing with exogenous actions.

A programming language for robot swarm named Buzz was presented in [70] which is dedicated for heterogeneous robot swarms. Buzz syntax mixes imperative and functional constructs and is inspired by dynamic languages as JavaScript, Lua and Python.

[71] introduces a method to use written natural language instructions to program 
assembly tasks for industrial robots, using the methodology of compilers development of designing a semantic and syntactic parsers.

Another contribution can be found at [72] The world has an increasing population of robots whose end users could benefit from being able to give them new tasks. Visual languages are a possible medium to accomplish this. We have taken a first step towards this through the realisation of Ruru, a visual language that enables novice programmers to create simple robot behaviours. It also addresses some inherent issues associated with robot software development. We plan to explore other domains, such as healthcare and agriculture, to facilitate the development of an end user robot programming language that can express more realistic real world robot tasks.

L-IRL (Lola Industrial Robot Language) [73] is procedural language for industrial robot programming. Offline and real-time programming system are main parts of robot programming system based on L-IRL programming language. System for robot programming is using object code as one of the main communication tools between different elements of the system. In this paper we have presented how object code of the compiler can be generated in the form of P code or XML code. This gives software solution that is compact, portable and easy to use with standardized object code that consist information for robot motion control. After generation of object code on the offline part of the system, it is sent to the real-time part of the system using CORBA protocol.

All the previous works implemented a new programming language to solve the task planning for the own robots. These languages had a formal definition, grammar and the implementation of lexical, syntactical and semantical analyzer. A brief explanation of how a programming language is developed is shown next.

\subsubsection{Formal languages}

A language can be seen as a system suitable for expression of certain ideas, facts and concepts [74]. For formalizing the notion of a language one must cover all the varieties of languages such as natural (human) languages and programming languages. It can be broadly seen that a language is a collection of sentences; a sentence is a sequence of words; and a word is a combination of syllables. It is observed that a formal learning of a language has the following three steps.

1. Learning its alphabet - the symbols that are used in the language.

2. Its words - as various sequences of symbols of its alphabet.

3. Formation of sentences - sequence of various words that follow certain rules of the language. 
Being step 3 the most difficult part. By this time, it is easy to assume that a word (string) is a sequence of the symbols that lies on an alphabet and are well-formed according to certain set of rules. In English, Italian, Spanish and other languages are based in the Roman alphabet along with punctuation marks such as commas, period, colon, semi-colon, and other special symbols like blank-space which is used to separate two words, but the way that words are built in each of these languages vary according to their own grammar. Thus, a formal language can be defined by means of a formal grammar such as regular grammar which consists of its formation rules.

Formal languages can be given as those string generated by a formal grammar, or described by a particular regular expression. The latest, is a sequence of characters that define a search pattern and are used basically in the lexical analysis of a compiler/interpreter.

\subsubsection{Grammars}

As stated previously, a formal grammar consist in a set of production rules to generate string from the language alphabet and are valid according to the language's syntax. The grammar does not describe the meaning of the strings. To obtain the meaning of a string is during the semantic analysis step that will be seen later.

According to [75] the grammars can be classified into types, this is known as the Chomsky hierarchy. Two important types of this hierarchy are context-free grammars (type 2) and regular grammars.

A context-free grammar should have a set of nonterminal symbols, a set a of terminal symbols and a set of rules. So the grammar is specified as a quadruple:

$$
G=(N, \Sigma, P . S)
$$

where

1. $N$ is a finite set of nonterminals

2. $\Sigma$ is a finite set of terminals

3. $S \in N$ is the start symbol

4. $P$ is a finite subset of $N \times V^{*}$ called the set of production rules. Here, $V=N \bigcup \Sigma$

\subsubsection{Compilers and interpreters}

A compiler is a computer program that converts a code written in a specific language (source code), usually a high level programming language like $\mathrm{C} / \mathrm{C}++/ \mathrm{C \#}$, Java, Python, 
into another programming language, normally a low level programming language like assembly, object code or machine code, in order to create an executable program. Meanwhile, the interpreter is other computer program that directly executes instructions written in a specific language without creating the executable application. Figure 2.8 shows the difference between compilers and interpreters.

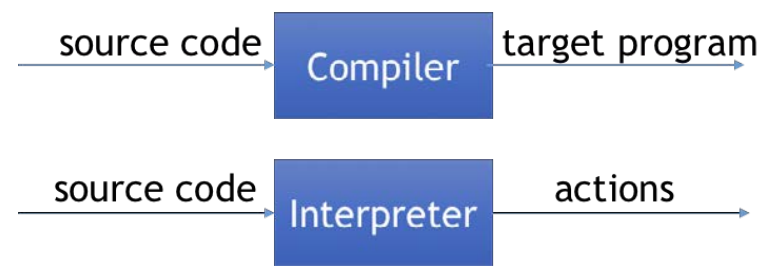

Figure 2.8: Compiler vs. interpreter

The similarity between a compiler and an interpreter is the analysis of the source code. This part breaks up the source program into constituent pieces and imposes a grammatical structure on them. It then uses this structure to create an intermediate representation of the source program. If the analysis part detects that the source program is either syntactically ill formed or semantically unsound, then it must provide informatice messages, so the user can take corrective action. The analysis part also collects information about the source program and stores it in a data structure called a symbol table, which is passes along the intermediate representation of the synthesis part. Observing the figure 2.9 is shown a typical decomposition of the analysis of an interpreter or a compiler.

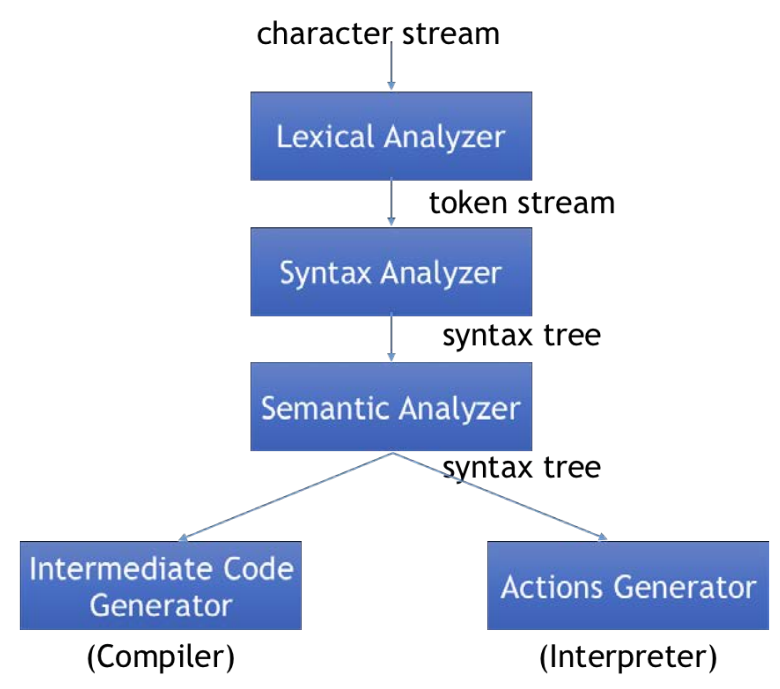

Figure 2.9: Analysis phase of a compiler/interpreter 


\subsubsection{Lexical analysis}

This is the first phase of an interpreter. The lexical analyzer reads characters from the input (source program) and groups them into meaningful sequences called lexemes [76]. For each lexeme, the lexical analyzer produces as output a token of the form:

(token-name, attribue-value)

producing a list of tokens that will be used on the subsequent phase, syntax analysis. In the token, the first component token-name is an abstract symbol that is used during syntax analysis, and the second component attribute-value points to an entry in the symbol tale for this token. Informatin from the symbol-table entry is needed for semantic analysis and code generation. For example suppose the next statement.

$$
\text { position }=\text { initial }+ \text { rate } * 60
$$

the characters in this assignment could be grouped into the following lexemes and mapped into the following tokens passed on to the syntax analyzer:

1. Position is a lexeme that would be mapped into a token (id, 1), where id is an abstract symbol standing for identifier and 1, points to the symbol-table entry for position. The symbol-table entry for an identifier holds information about the identifier, such as its name and type.

2. The assignment symbol $=$ is a lexeme that is mapped into the token $(=)$.

3. initial is a lexeme that is mapped into the token (id, 2), where 2 points to the symboltable entry for initial.

4. + is a lexeme that is mapped into the token $(+)$.

5. rate is a lexeme that is mapped into the token (id, 3 ), where 3 points to the symboltable entry for rate.

6. * is a lexeme that is mapped into the token $(*)$.

7. 60 is a lexeme that is mapped into the token (number, 60).

Blanks separating the lexemes are discarded by the lexical analyzer.

\subsubsection{Syntax analysis}

According to [76] the parsing is the process of determining how a string of terminals can be generated by a grammar. To solve this issue, a parse tree must be constructed. For any context-free grammar there is a parser that takes at most $\mathbf{O}\left(n^{3}\right)$ time to parse a string of $\mathrm{n}$ terminals, being cubic time really expensive. For real programming languages, parsing 
could be solved by using linear-time algorithms, performing a left-to-right scan over the input, building the parsing tree as they go.

Most parsing methods fall into one of two classes, called the top-down and bottomup parsers, being the first the most used. In top-down parsers, construction starts at the root and proceeds towards the leaves, while in bottom-up parsers, construction starts at the leaves and proceeds towards the root.

The grammar 2.1 generates a subset of a statement in $\mathrm{C}$, where the terminal if and for are terminal elements and also are keywords, the expr represents expressions, so the the figure 2.10 represents the top-down parsing tree which is done by starting with the root, labeled with the starting nonterminal stmt, and repeatedly the following two steps.

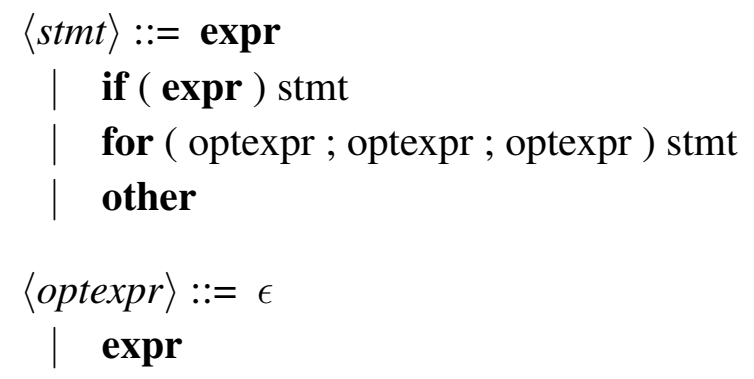

Grammar 2.1: A grammar for some statements in C language

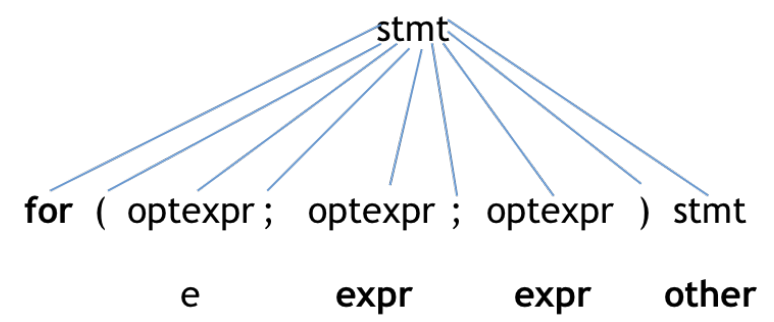

Figure 2.10: A parse tree according to the grammar 2.1

1. At node $N$, labeled with nonterminal $A$, select one of the productions for $A$ and construct children at $N$ for the symbols in the production body.

2. Find the next node at which a subtree is to be constructed, typically the leftmost unexpanded nonterminal of the tree.

For some grammars, the above steps can be implemented during a single left-to-right scan of the input string. The current terminal being scanned in the input is frequently referred to as the lookahead symbol. Initially, the lookahead symbol is the first leftmost, terminal of the input string. 


\subsubsection{Semantic analysis}

The semantic analyzer uses the syntax tree and the information in the symbol table to check the source program for semantic consistency with the language definition [76]. An important part of semantic analysis is type checking where the interpreter checks that each operator has matching operands. For example, many programming languages definition require an array index to be an integer; the compiler must report an error if a floating-point number is used to index an array.

The language speciification may permit some type conversions called coercions. For example, a binary arithmetic operator may be applied to either a pair of integers or to a pair of floating-point numbers. If the operator is applied to a floating-point number and an integer, the interpreter may convert or coerce the integer into a floating-point number. 


\section{Doris architecture}

Programming a robot is not an easy task since there are many issues that need to be solved, such as the localization of the robot, its movements, the reading of information from complex environments, the processing of some eventual conditions, the interpretation of an input and the appropriate answer to this input and so on and so forth. Once they are solved, these tasks need to work synchronized.

This dissertation presents a control architecture that involves both, hardware and software proposals, in order for Doris to be able to work as a successful tour-guide robot in museums. Therefore, five layers have been developed: a hardware, a logic, a link, a task planner and a user layer. The hardware layer communicates to the logic layer through a USB and a RS232 or Ethernet; and the remaining layers communicate with each other through pipes, shared memory, messages and sockets.

When it comes to Doris' hardware, the robot is equipped with a mobile platform. Prior to this dissertation, a skeleton placed on this platform was added to the robot in order to provide it with human appearance. Later, the head was attached to the skeleton so that interaction with the environment could be possible, as well as additional sensors such as the RFID and an omnidirectional camera, which are used for localization, navigation and interaction.

Regarding software, there is a proposal of a client-server application which includes multiple threads which communicate with each other in order to achieve an excellent performance between internal processes and between the robot and the people.

This chapter covers different areas of the architecture proposed. Firstly, it starts with a general view of the architecture, providing a description of each module. Secondly, the hardware modifications are explained and a brief explanation of the sensors used and the way in which they work is given. Then, the developed software is described. As the language used is $\mathrm{C}++$, classes diagrams are developed to describe the relationships between each of the classes used in the architecture. Moreover, an explanation about how 
Doris' face was built and the ways to control it are given together with an explanation about how its robotic arm works. Furthermore, as the robot needs to communicate with the environment, a Text To Speech (TTS) module was added, so a brief explanation about how it is connected with the architecture and a description of the planners added to the architecture are provided too.

Doris is a mobile robot conceived to work in dynamic indoor environments and it is intended to interact with people. It was built as a way to upgrade Blacky and Urbano, two robots that have been developed at the Centre of Automation and Robotics in the Universidad Politécnica de Madrid in recent years.

\subsection{Doris control scheme}

The architecture proposed takes into account different issues that can be presented in a complex environment as a museum or a laboratory. Those issues might be:

- People moving around.

- People speaking.

- People's children trying to interact with the robot.

- Objects in the middle of a room.

- Different room shapes inside the same museum.

- Hallways.

As seen before, people are the main problem of a complex environment, this implies the development of an interface for interaction. And the second main problem is the environment itself, so the robot needs to know its own location to achieve different interaction in different places.

The architecture proposed handles these kind of problems by subdividing the issues in several subsystems [77] [78]. Figure 3.1 shows these subsystems distributed along different layers. Each block on the figure details a task that is asynchronously under execution on a different thread, which means that in the higher level of the architecture, there is a task handler.

- Hardware Layer: starts connections with different devices installed on the platform (encoders, bumpers, sonars, cameras, laser, etc), as well as with USB and ethernet devices, at a level with permissions for read/write data provided by superior levels. e.g. the robot position, the speed at which the robot must move, etc. 


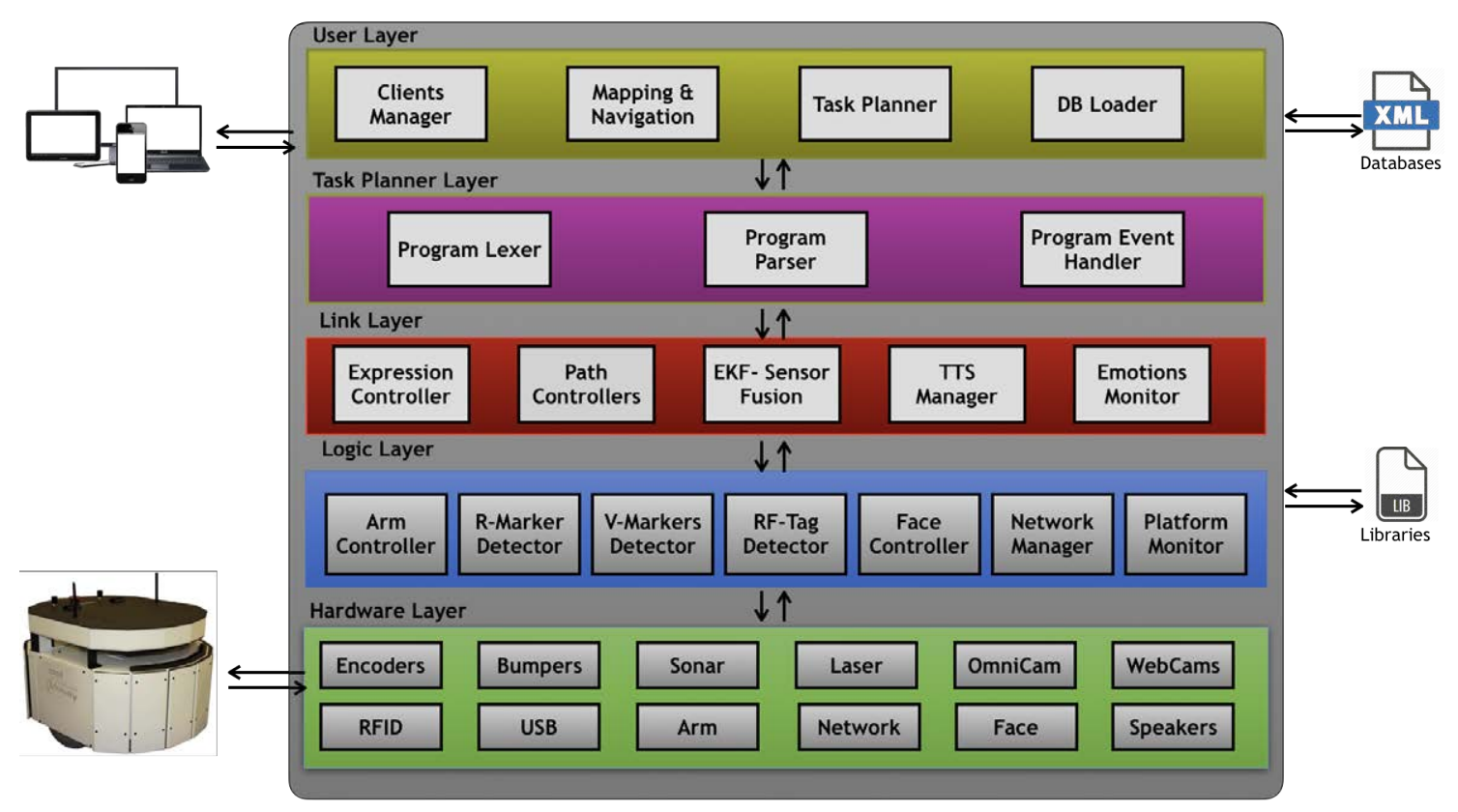

Figure 3.1: Software architecture of Doris

- Logic Layer: at this level, data sent from first layer are processed, for later use by the immediate superior layer, e.g. Data from encoders is translated into differential polar coordinates $[\Delta \rho(k) \Delta \phi(k)]^{T}$ for odometry, and then converted into an oriented position $[x(k) y(k) \theta(k)]^{T}$.

All of the modules in this layer are threads that are launched since the application is executed, for example: R-Marker is a thread dedicated to detect the reflective landmarks, the V-Markers is other thread detecting the visual markers, and the RFTag detection, is a thread for detecting the RFID tags.

It also turns on the server communications with a networked camera and an RF device.

- Link Layer: instructions for robotic movements are generated at this level of Doris' programming. The localization task that takes place on this layer processes data from the logic layer, by using odometrical information and data from sensor detector tasks. Emotion and expressive tasks are also performed, where they can command the face and/or the arm. A movement monitor is also included to supervise which actions Doris must perform depending on the localization.

- Task Planner Layer: This layer links all the modules from lower layers with a program developed by the robot user. Hence, this layer contains a lexer and a parser for the program interpretation, and the program event handler which connects with the events dispatched by the modules from the link layer.

- User Layer: Client communications are performed at the user layer along with database loading and the navigational commands are inputted by the user. Doris 
can manage up to ten users at the same time, but only one can have full access to perform tasks.

\subsection{Hardware description}

As stated before, the architecture proposes modifications in hardware. The initial state of it can be found in the platform, designed by Adept MobileRobots, is a differential steer mobile robot, which has built-in sensors (bumpers, sonars, and laser), all connected to the serial port of the internal computer. This computer has USB ports, ethernet and a WIFI connection. Figure 3.2 shows the platform.

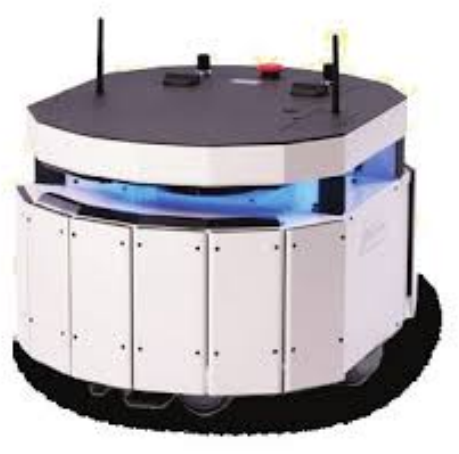

(a) Platform

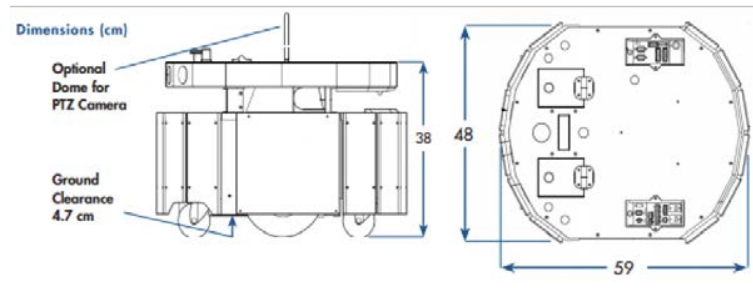

(b) Platform schematic

Figure 3.2: Doris platform

The skeleton 3.3 was developed in a previous work, it is attached to the mobile platform, made of methacrylate, holds up speakers, the RFID antennas, a USB HUB, a PoE switch and the robot's head. The torso is designed to replicate human physical appearance. In the future, an arm will be attached to develop pick and place tasks.

From this state, the architecture proposes a new head design with more degrees of freedom (DoF) to achieve a more realistic interaction. The head is attached to the skeleton, with 20 DoF can show different expressions and can be also selected by the robot user. The robot is also speech capable and its mouth will synchronize with the sound that is emitted; a combination known as viseme-based acoustic speech, that is shown in figure 3.4. This will be explained in detail in section 3.5.1

The information gathered from all sensors, is used for indoor localization. The joint sensor operation will be detailed in chapter 4. 


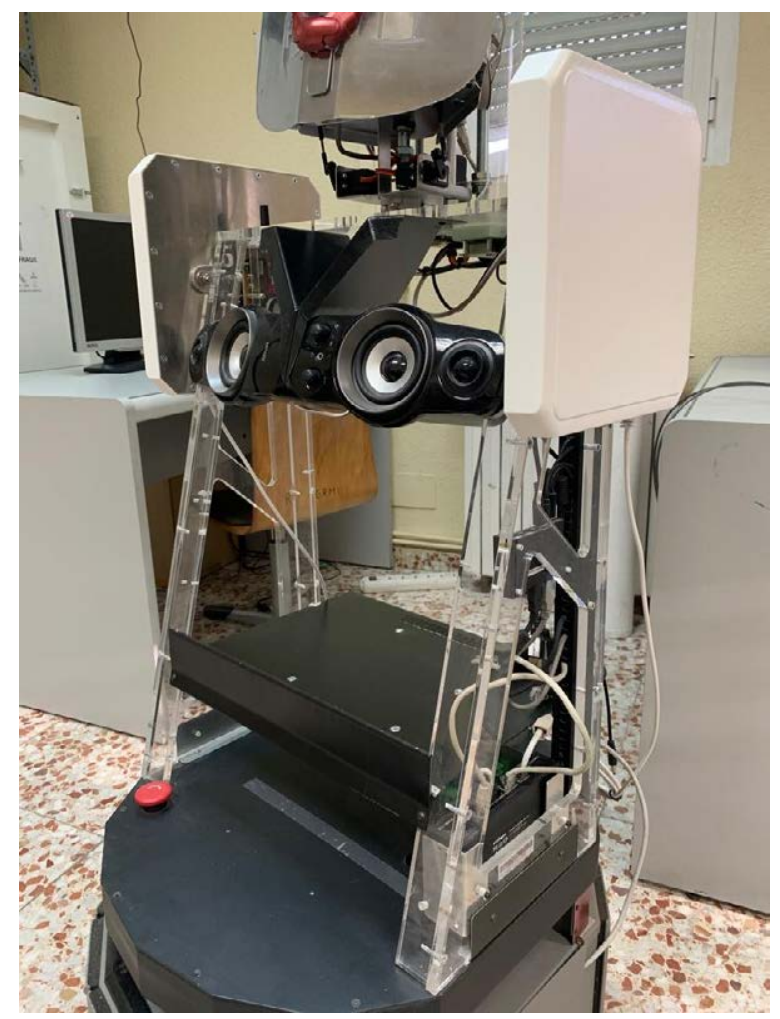

Figure 3.3: Doris skeleton

\subsection{Software description}

The software, developed to achieve a full autonomy for Doris in different environments, has been designed in $\mathrm{C}++$. This programming language is used during this dissertation because it is language by excellence for object modelling. But, why is $\mathrm{C}++$ used instead of $\mathrm{C}$ or Java or even Python? $\mathrm{C}++$ is the programming language more capable to manage pointers and functions pointers for callbacks and events. From the $\mathrm{C}++11$ version, a lot of functionality has been added making more easier to connect to the different devices, including the platform which the whole library to connect to the robot was developed in $\mathrm{C}++$.

The software works as a Client-Server application, enabling direct communication between Doris (server) and the (client) that controls it. Nowadays, there are three different types of client applications for Doris: Web, Desktop, and Mobile devices. The type of clients will be discussed in chapter 6. Each block on the figure details a task that is asynchronously under execution on a different thread which means that in the higher level of the architecture, there is a task handler.

When working with threads it is so common that each one needs to communicate with other threads. To solve this problem some common methods are:

- Shared memory allowing to each thread to share a specific zone of memory to read 


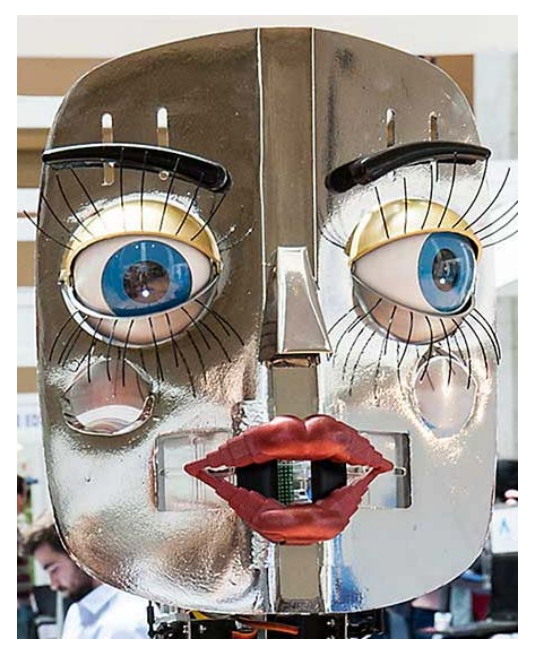

Figure 3.4: Doris head

\section{or write information}

- Pipes and message queues, allowing to send and receive information sequentially.

- Sockets, which allows communication with other processes that are being executed in other computers, but this depends of the local or wide area network.

In the architecture proposed, shared memory and sockets are the most used method of communication. So, this will allow that each thread runs as a publisher/subscriber process. It can be inferred that similar resources to this type of application are available like the Robotic Operating System (ROS).

ROS is a middleware platform widely used in robotics to develop modular applications, being also a publisher/subscriber system. But, ROS has many inconvenience, one of them is the complexity of the messages, too many unwanted packages, and the most important memory leaks. Doris is intended to work for several hours. and the onboard computer does not have enough RAM memory and no GPU. For this very important reason, the decision to not use ROS was taken, and only use the software needed to communicate with other components.

This dissertation proposes the implementation of the next classes shown in figure 3.5. These classes will achieve the proposed multi-threaded client-server model. These classes are:

- RobotNode: this is a pure abstract class that is the responsible for connection with the platform and obtain all the information from encoders, to connect with the laser, the sonar, bumpers and process all other data provided from the platform.

- TcpSocketServer: this is another pure abstract class that is the responsible for connection with clients using sockets or websockets. Also establishes a priority queue to handle different requests from users. 
- GeneralController: this class is created by inheritance from RobotNode and TcpSocketServer and it is the principal to receive the messages from clients and other classes. Also handles the shared memory between the processes generated, and handles the maps and the configuration of the robot. This class also sends raw data to the clients connected over an UDP socket connection. This data can be, laser data, the position, velocities, etc.

- MapSector: is a class that handles the information from the current sector where the robot is at the current moment.

- RNRecurrentTask: is an abstract class that allows to create a new thread and allow to publish information and also allow other threads to subscribe to the thread.

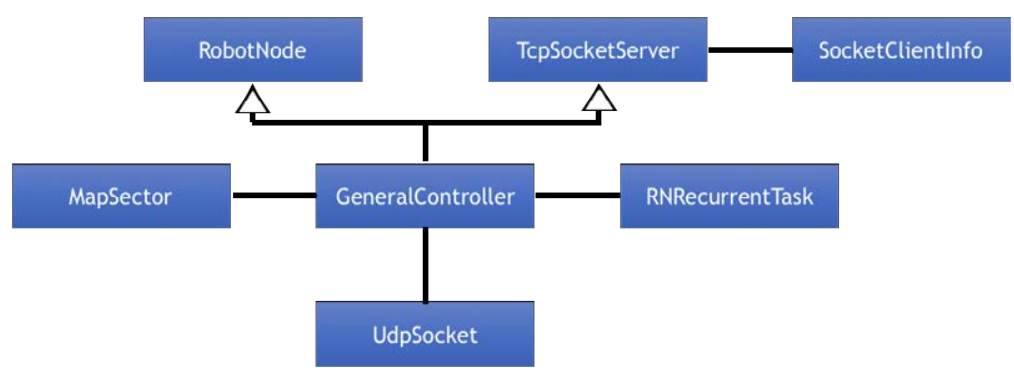

Figure 3.5: Class diagram of principal classes

The RNRecurrentTask detailed in the class diagram shown in figure 3.6 derives to other threads which are:

- RNOmnicameraTask: it is a class responsible to detect the QR codes placed on walls, publishing information concerning the position, map, sector and id of the visual landmark

- RNLaserTask: is a class commissioned to publish the raw information acquired from a 180 degrees scan, to publish the detected reflective landmarks, and keep a security area when objects are detected below $0.5 \mathrm{~m}$.

- RNLocalizationTask: is a class which implements the Extended Kalman Filter and subscribes to the RNOmnicameraTask and RNLaserTask threads.

- RNEmotionTask: is a class commissioned to handle the emotions given a specific input publishing different results like the face state, the answer to the input text and the modulation of the voice.

- RNGestureTask: this class handles the face of Doris. This thread is always expecting the resulting emotion, which will make Doris have different expressions according to it. 
- RNDialogsTask: this task is commissioned to handle the text input and to provide a coherent answer given by the resulting emotion.

- RNRfidTask: this task detects all the RFID tags placed on the environment and publish all of them.

- RNArmTask: this task handles the arm positioning according to some pre-established actions.

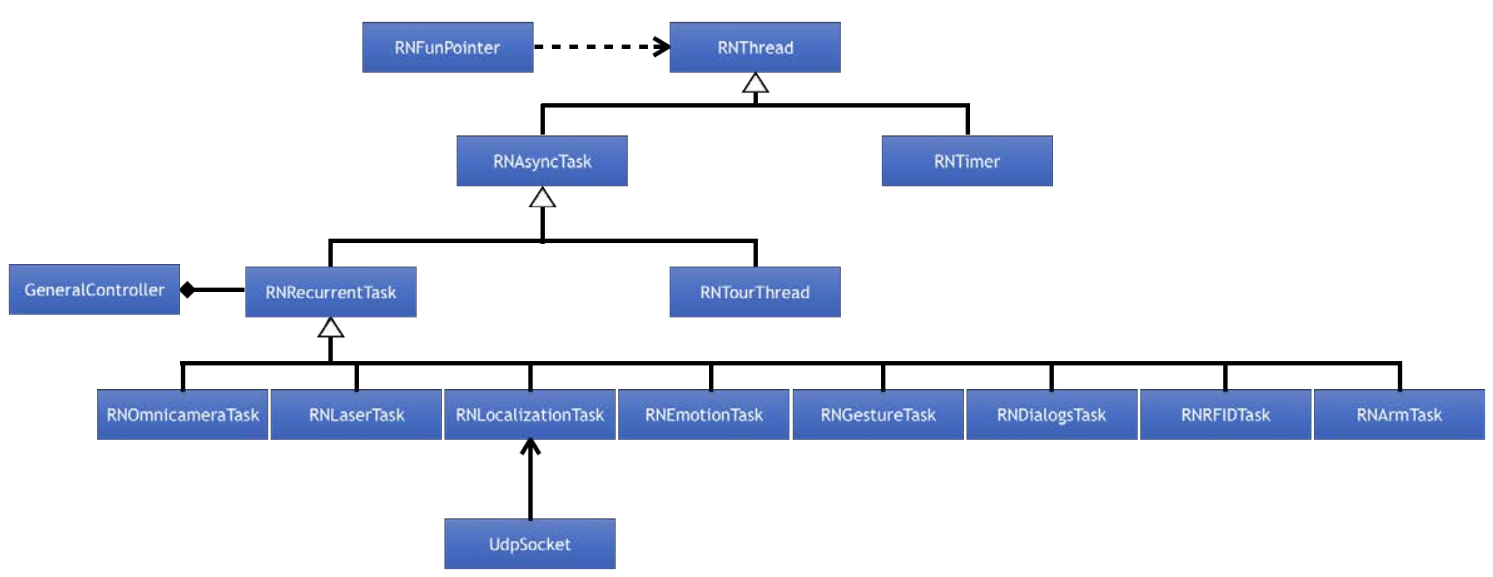

Figure 3.6: Class diagram of threads

\subsection{Perception}

In diagram 3.1 there is a list of components for perception of the environment like sonar, bumpers which are installed in the mobile platform but the are not currently used in schema, being reserved for future applications. Other components like the cameras, RFID and laser range finder are proposed to be used in this dissertation for localization and navigation. Laser has been widely used for SLAM, but as the proposals of this dissertation is to avoid the use of SLAM technique, the laser is decided to be used for localization using the Extended Kalman Filter.

Also an omnidirectional camera is used for localization. The importance of this camera is due that it has a 360 degrees of field of view. In this proposal, the camera will be placed behind the head, attached to a base, and this base will be attached to the skeleton.

In order to use both sensors for the same purpose, a sensor fusion is proposed in this architecture to locate the robot. The sensor fusion is needed in case of one of the sensors is unable to be used in specific moments or conditions.

It was also decided to install a RFID system over the platform. The proposed architecture contemplates the use of semantics to describe the environment of a museum. 
In order to achieve this goal, RFID tags will be placed in the environment and also assigned to the people inside the environment. The sensors are explained next.

\subsubsection{Laser range finder}

The LMS200 is a non-contact measurement system in stand-alone operation for industrial applications. The system scans its surroundings two-dimensionally with a radial field of vision using infra-red laser beam (laser radar). The system require either reflectors nor position marks. The laser measurement systems are used for area monitoring, object measurement and detection and determining positions.

Two field sets, each with a maximum of three monitoring fields, can be configured in the LMS200 for area monitoring applications. One field set is always active. Each monitored field is assigned a switching output (quiescent voltage typical $24 \mathrm{~V}$ DC). Special devices offer relay outputs. Measurement data output can take place parallel to area monitoring. Standard solutions are available for object measurement, e.g. measuring the volumes of packages and pallets; volume flow measurement for bulk materials, etc. The measured data of the LMS200 can be individually processes in real-time with external evaluation software for determining positions and other measurement tasks.

Some features and advantages of the LMS200 are:

- Non-contact optical measurement, even over longer distances

- Rapid scanning times, thus measurement objects can be move at high speed

- No special target-object reflective properties necessary

- No reflectors and no marking of the measurement objects necessary

- Backgrounds and surroundings do not have any influence on the measurements

- Measurement objects may be in any position

- Measurement data is available in real-time and can be used for further processing or control tasks

- Active system: no illumination of the measurement area required

The design of the LMS200 measurement system consists in a laser scanner, evaluation electronics, measurement and evaluation software (firmware with integrated area monitoring), a data interface (RS 232/RS422, switchable), a digital switching input and three function indicators (LED) 
The LMS200 operates by measuring the time of flight of laser light pulses: a pulsed laser beam is emitted and reflected if it meets an object (figure 3.7). The reflection is registered by the LMS200's receiver. The time between transmission and reception of the impulse is directly proportional to the distance between the LMS200 and the object (time of flight).

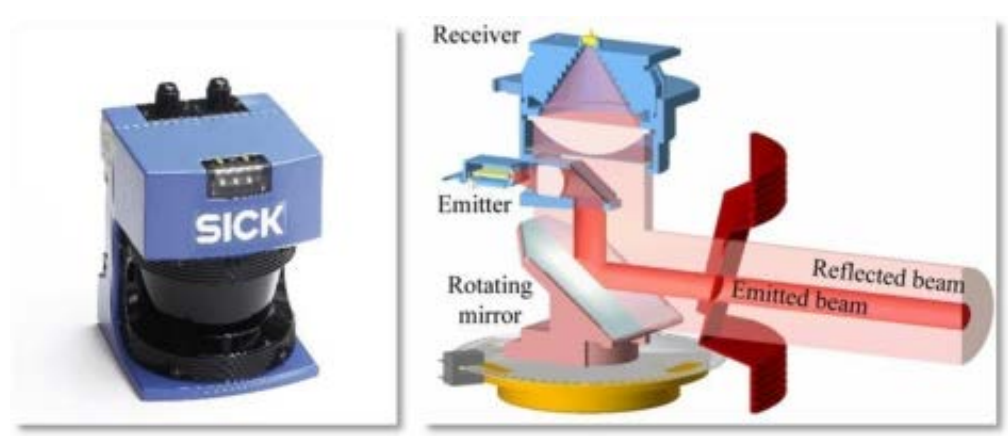

Figure 3.7: LMS200 operating principle

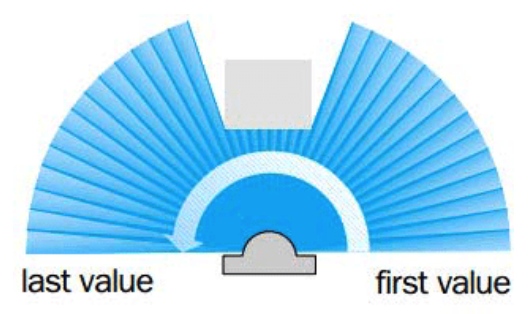

scanning angle $180^{\circ}$

Figure 3.8: LMS200 direction of transmission and 180 degrees of scanning

\subsubsection{Omnidirectional camera}

It is a camera having a field of view that cover 360-degree in the horizontal plan, being commonly used in robotics. The omnidirectional camera captures light falling onto the camera focal point through a hemisphere. In contrast to an ideal omnidirectional camera which captures the light from all points, covering a full sphere (360-degrees horizontally and 360-degrees vertically). In figure 3.9 is shown how the operating principle works. 


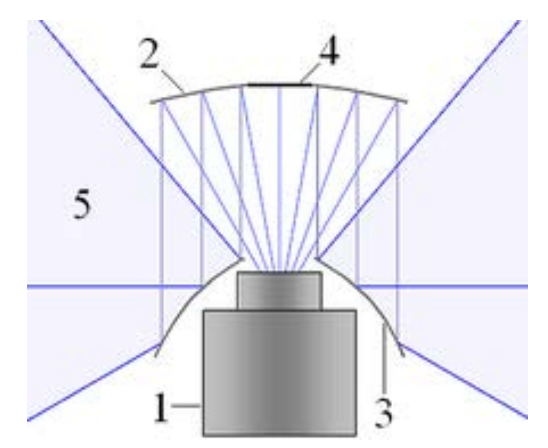

Figure 3.9: Omnidirectional camera operating principle

1. Camera

2. Upper mirror

3. Lower mirror

4. Black spot

5. Field of view (light view)

The camera used in this research was the Mobotix C25 indoor ceiling omnidirectional camera.

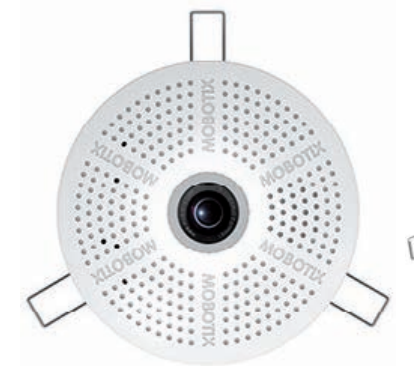

(a) Front view

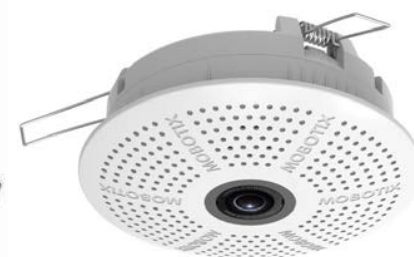

(b) Bottom view

Figure 3.10: Mobotix C25 Omnidirectional camera

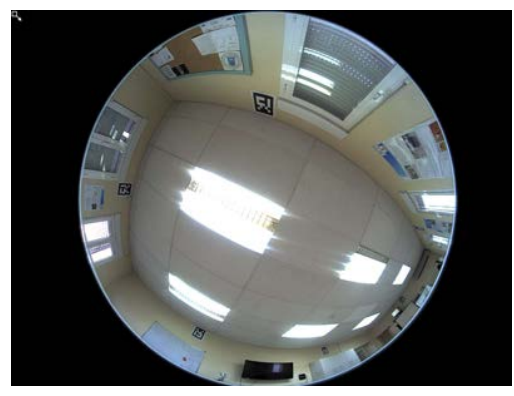

Figure 3.11: Image taken by the omnidirectional camera 


\subsubsection{Radio Frequency Identification}

The basic function of an RFID system is to automatically retrieve the information that has been previously inserted into specific integrated circuits, as seen in figure 3.12. RFID systems were first developed in automatic identification laboratories as a natural evolution of the different technologies they use. Different automatic identification systems use different methods to transmit the identifying information. For example, bar code technology uses light as the transmission media, while RFID systems use radio waves [79].

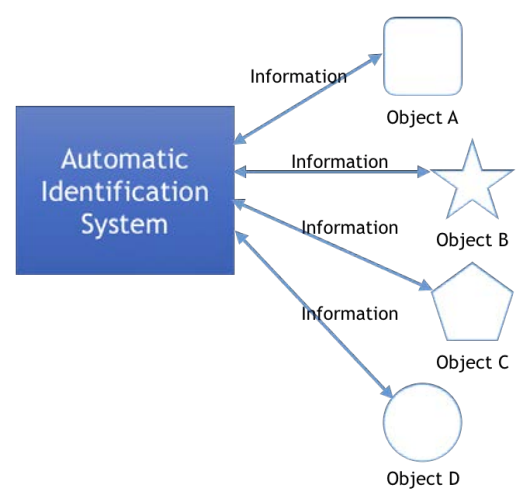

Figure 3.12: Information transfer in an automatic identification system

The devices that store and carry the information are called transponders or tags and are shown in figure 3.13. Although the industry commonly refers to them as tags mainly due to their physical shape and the fact that they are mostly used to tag pallets or cases of goods, the name transponder reflects better the function of these devices. The device that is used to capture and transfer information is commonly called a reader, because in earlier RFID systems they were only able to read the information sent by the transponders. However, with the development of new functions and applications of RFID systems, the name interrogator reflects better the function of this subsystem. Therefore, it will be used the names transponder and interrogator when referring to these elements. Transponders must have the circuitry needed to harvest power from the electromagnetic fields generated by the interrogator, the necessary memory elements, as well as the different control circuits.

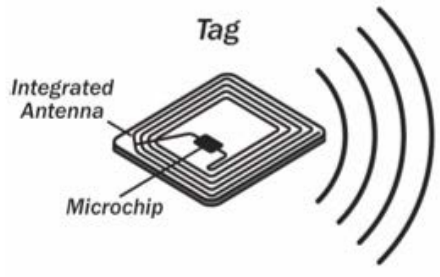

Figure 3.13: Sample of a tag used in RFID systems 
The applications on guiding robots using RFID systems in narrow areas (through hallways or negotiating a door) are really low, so a certain degree of precision is needed that is not provided by this type of system, but it can be useful for other localization tasks as global positioning.

The RFID Impinj R220 reader system (figure 3.14a) installed in Doris has two right circular polarity antenna S8658PR (figure 3.14b), from which three different measures can be obtained:

1. RSSI: Received Signal Strength Indicator, which provides an estimate of the tag backscatter signal power received $P_{R}$ in Watts.

2. RF phase angle: represents the phase rotation angle over distance

3. Doppler Frequency: is the shift of the received signal at the reader, due to relative motion between the reader and the tag. This feature is helpful for determining the tags that are in motion (people moving).

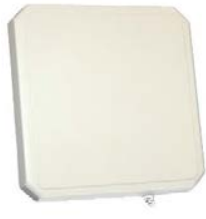

(a) Antenna

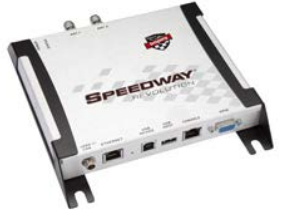

(b) Impinj R220 Reader

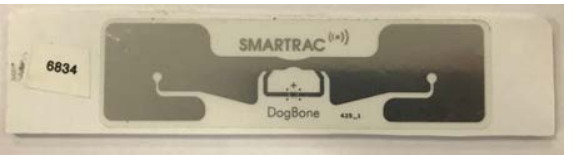

(c) RFID tag dogbone

Figure 3.14: RFID System installed on Doris

\subsection{Actuators}

One of the proposals for the architecture is that Doris needs to interact with environment, so this interaction can be achieved from different points of view. Some humans tend to be more expressive with the body than with the face, or the other way around, or even both. As the Doris' body does not have the ability to move, a robotic face and a robotic arm were added to achieve this type of interaction. In this section the interfaces added architecture are explained.

\subsubsection{Face controller}

This architecture proposes that Doris must have a Human-Machine Interface for human interaction. The most common characteristic of a social interactive robot is having a face. This face must be human-accepted, being able to avoid the uncanny valley, this means 
that the robot should not create rejection among humans due to a similarity to a human face.

The first proposal of this dissertation is to design a human face is shown as in the figure 3.15. This design states that the face contains 20 degrees of freedom (DoF) distributed as:

- 2 DoF per each eye (2) to move the eye up and down and from right to left and being able to look up, right, left or down.

- 2 DoF per each eyelash (2) to move it up and down from the corners being able to have a normal appearance or to frown.

- 1 DoF per each eyelid (2) to open or close the eyes.

- 2 DoF for the lips to open or close the mouth.

- 2 DoF for each commissure (2) for being able to replicate smiles or sad faces.

- 1 DoF for the cheeks for being able to blush.

- 3 DoF to move the head up and down, right and left and to lean the head from one side to another.

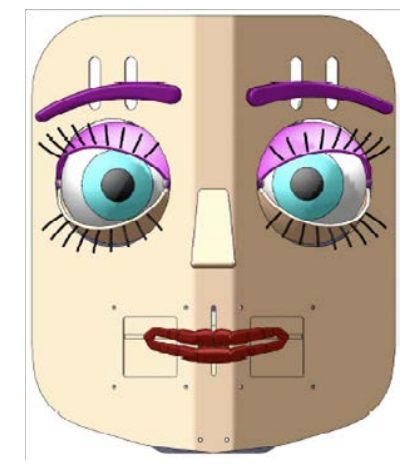

Figure 3.15: Doris initial design face

The face is controlled by servos with the ability to locate in any desired angular position in a specified range. This position is controlled by a pulse train. This motors are positioned by 2 Pololu min Maestro Controller card as shown in the figure 3.16 


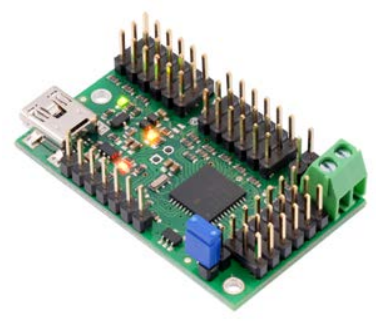

Figure 3.16: Pololu mini 18 maestro controller

\subsubsection{Arm controller}

The second proposal of this dissertation is that Doris must have a robotic arm to interact with people and in a future to perform task to pick and place in certain area. To perform these task some hardware needs to be installed in order to achieve the arm control more easily.

The arm has six degrees of freedom distributed as next:

- Two DoF placed in the shoulder, allowing a turn each one, one turn over the plane $y-z$ and another turn in the plane $x-z$.

- On the elbow, a DoF allows the turn of the arm around the axis of the arm itself. Another DoF allows the forearm to raise and go down.

- Finally, two other DoF are located in the wrist allowing the hand turns around the forearm and to raise and go down.

Also has other 3 degrees of freedom located in the hand allowing to move each finger individually. The shoulder allows an elevation of $60 \mathrm{deg}$ and to turn up to 45 degrees. The elbow can turn up to 120 degrees and raise up to 120 degrees, finally the hand may turn 180 degrees and raise and go down 180 degrees also. In Figure 3.17 is appreciated some limits of the robotic arm. 


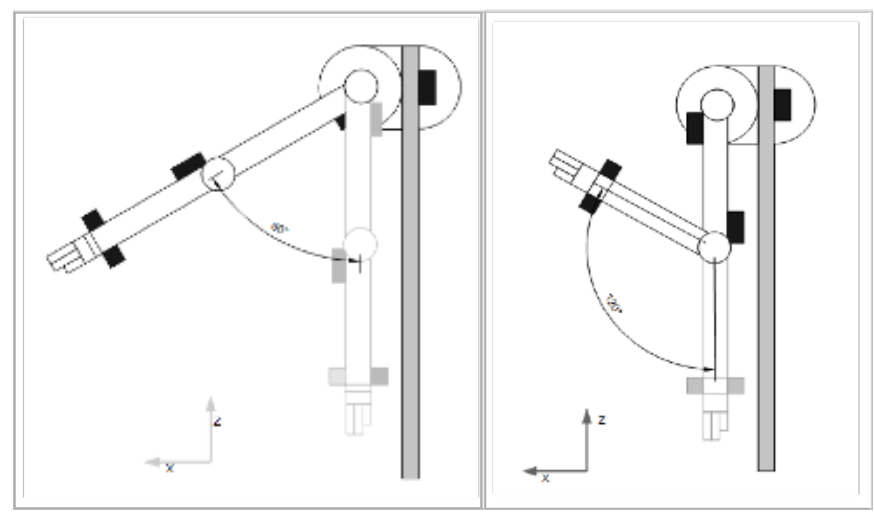

(a) Shoulder raising

(b) Elbow raising

Figure 3.17: Robotic arm model

The Dynamixel motors from Robotis are used to achieve control in the arm articulations. The MX64-AT (figure 3.18a) model is used for the shoulder and the elbow motor, meanwhile the AX-12A (figure 3.18b) is the model used for the wrist motors. These were selected due to the high torque supported, the high precision and the high number of parameters the motors have.

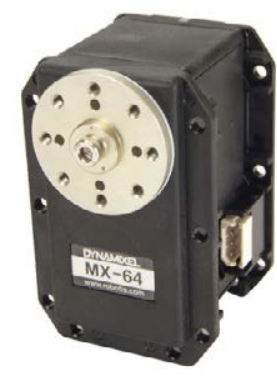

(a) MX64-AT

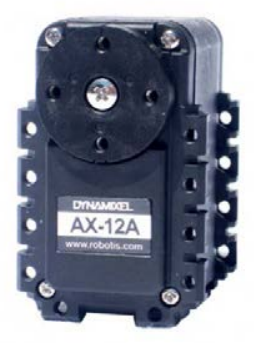

(b) $\mathrm{AX}-12 \mathrm{~A}$

Figure 3.18: Dynamixel Motors

These motors communicate over a TTL (Transistor-Transistor Logic) connection, which is an async communication method UART (Universally Asynchronous ReceiverTransmitter). Also the motors can connect each other in daisy chain, so each motor must have an unique ID as shown in figure 3.19 . 


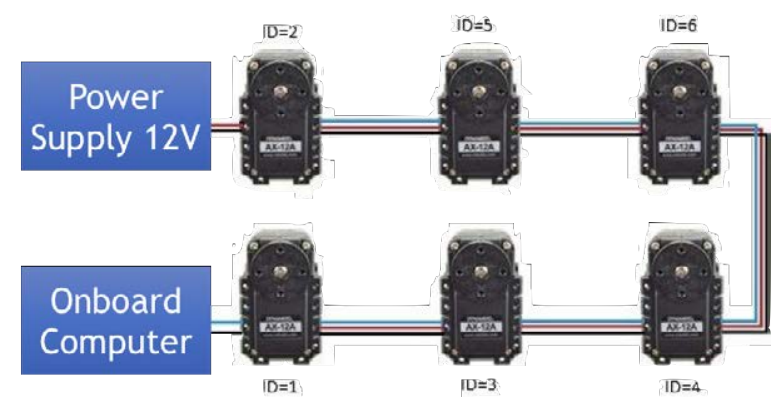

Figure 3.19: Connections between each dynamixel motor

For the final connection to the onboard computer on Doris, the USB2Dynamixel (figure 3.20) is used. This device allows the RS-485 or RS-232 connections

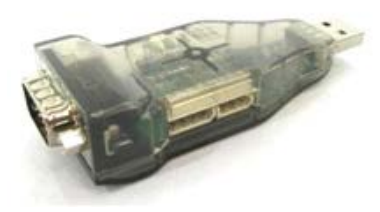

Figure 3.20: USB2Dynamixel converter

For the motors placed at the hand, the pololu mini maestro controller explained above is used.

All these components can be modelled from the software point of view. Figure 3.21 shows the classes developed to achieve the arm control.

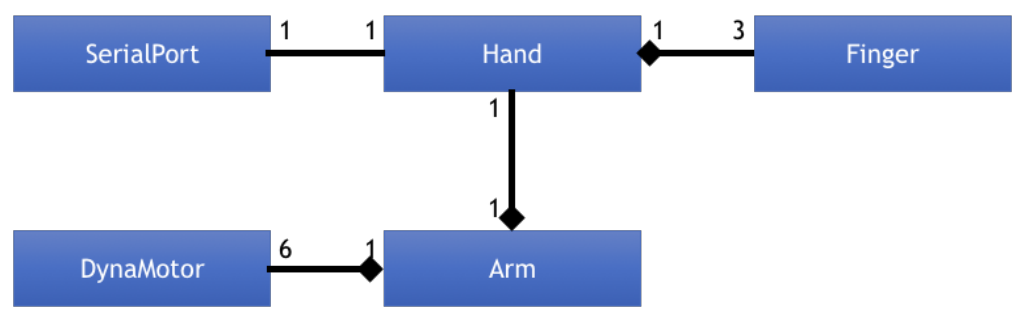

Figure 3.21: Doris arm class diagram

\subsubsection{Text To Speech Manager}

Doris has an emotional expressivity and to get real interaction, a verbal communication must be achieved, being this the synchronization of the sound emitted by Doris with the lips movement. This will result in some kind of natural speaking, being this more accepted by humans. 
The achievement of the lip syncronization with the voice emitted by Doris starts by conceiving the relation between all the elements involved, which are the text that is going to be emitted by the Text To Speech (TTS) module and the speed at which the mouth servos might move. The class diagram of the figure 3.22 explains this relation.

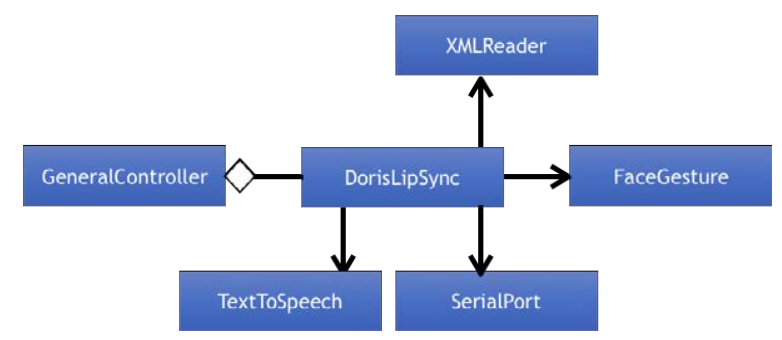

Figure 3.22: Class diagram of the text to speech manager

The classes work as next:

- DorisLipSync: this class is committed to receive the text and then convert it into visemes to then send it over the serial port to the pololu maestro controller and positioning the mouth in the desired position.

- SerialPort: this class is the reposible to communicate with al the serial devices connected to the robot. In this particular case 2 instances are made. one handles the doris face and the other to connect with the TTS module.

- TextToSpeech: this class handles the data sent to the TTS module.

- FaceGesture: contains information of the robotic face, hence also contains information of the mouth servos.

- XMLReader: reads information of the visemes and the face which is stored in databases written in XML.

The Text to Speech (TTS) device, which is initialized in the DorisLipSync class, it is configured with an predetermined setup as voice rate, pitch and volume, and this configuration will be modified by the Emotion Task explained in chapter 5 . After the configuration of the TTS device, it keeps waiting for some text to be emitted. The TTS device used is the Emic 2 as depicted in figure 3.23 a, helped by a USB to serial converter FTDI232 depicted in figure $3.23 \mathrm{~b}$. 


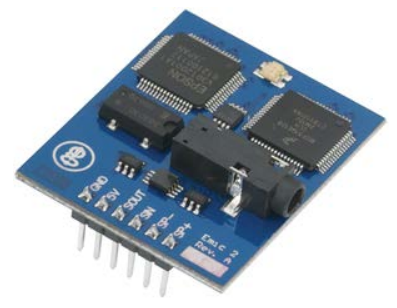

(a) Emic 2 Parallax

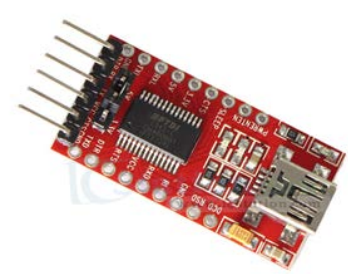

(b) FTDI232

Figure 3.23: Hardware used for text to speech

\subsection{Conclusions}

Several works concerning different architectures were mentioned in chapter 2, but most of them were focused on a single point of view such as localization, navigation or interaction, but not on all of them at the same time.

Throughout this chapter, the architecture proposed takes into account some parts from all the works mentioned in this chapter but improving them and adding some new ideas. The proposals of this architecture are made in hardware and software.

Regarding hardware, the architecture proposes a robot appearance that tends to be accepted, taking into account the avoidance of the uncanny valley. One of the ideas to avoid this issue consists in adding emotions to the robot when interacting with it. Another issue taken into account is the added sensorial system, which improves localization, navigation and planning, where the combination of an omnidirectional camera, RFID system and the laser range finder achieves a better performance concerning architecture modules.

Concerning the software, architecture proposes a multithreaded client-server system, which avoids the use of other types of systems like $\boldsymbol{R O S}$. It is very important to reduce the third party software to the minimum in order to have a better control of all modules. The language that is going to be used for the development of this dissertation is $\mathrm{C}++14$, so much work needs to be performed. 


\section{Localization, navigation and control system}

The task planner, previously mentioned, is another proposal of this doctoral thesis, which is responsible for indicating the set of actions that Doris must perform in a museum. This actions or tasks are the set of movements that Doris needs to do in order to reach a point of interest inside the museum. A route must be traced with a designed path planner in order to reach this point of interest, and in order to make the robot follow this path, two main components must be developed: the controller and the state observer, being this last one Doris' oriented position in the plane.

According to what was mentioned before, Doris must have a state observer which is based on an indoor positioning system (IPS) that allows the robot to locate inside the museum. Doris' localization is based on a sensor fusion, a subject that is approached by using the methodology published in [78] and [77] with the detection of reflective beacons by means of a laser range finder, and with the detection of visual markers by means of an omnidirectional camera. The information provided along with the information contained in the semantic map is fused into an Extended Kalman Filter.

The reflective landmarks are detected by using a LMS-200 laser range finder. These landmarks are circular shaped and its design is an ad-hoc model proposed in this dissertation. Its detection was achieved by following certain conditions for classifing and detecting the center of the landmark by applying the Law of Sines and using it as a model in an iterative minimum squared error estimator.

The visual markers are detected by using an omnidirectional camera Mobotix C25. The fiducial markers proposed for this dissertation are a matrix of 6 rows and 5 columns to avoid orientation issues, which are placed at $275 \mathrm{~cm}$ from the ground to try to avoid occlusions and to get the maximum numbers of visual markers. While the camera can get up to 5 markers at the same time the laser can perceive at least 2.

Concerning the reactive control proposed in this dissertation, two fuzzy controllers were developed. One of the controllers is a speed controller developed to move the robot 
from one point to another smoothly. Another controller is used in hallways, where the robot must keep in narrow areas to avoid collisions with other objects, which is similar to people's typical behaviour

The path planner for Doris navigation is based on two simple strategies. Another proposal of this dissertation is to design a semantic map of the environment that will be used for navigation. This map is subdivided into sectors and each sector reflects in points of interest where the robot must navigate. Different approaches, hich are based on Dijsktra or other planners based on graphs representation, have been made to solve the navigation and planning problems.

The development of all these proposals will be detailed throughout this chapter: the state observer based on the extended kalman filter, the control strategies based on fuzzy logic and the trajectory planning based on a $\mathbf{A}^{*}$ and branch and bound algorithms. Finally, at the end of this chapter, the experiments, results and conclusions obtained will be presented and the way in which they help to develop the architecture presented in this dissertation.

\subsection{Localization}

Doris localization is based on a sensor fusion derived from the Extended Kalman Filter. The sensors to be used are a Laser Range Finder, which provides a 180 degrees point cloud, and an Omnidirectional Camera, which provides an image of 360 degrees of field of view.

In this section will be explained how the information from the omnidirectional camera and the laser are obtained. Although the RFID is not used for localization, the form of how the information is obtained is also shown. Then the error estimation of each sensor is explained, including the information of the odometry which is provided by the platform used by Doris. Later, a brief explanation of the Extended Kalman Filter and the sensor integration is given.

\subsubsection{Obtaining information from sensors}

There are several studies in which reliable accuracy of robot localization is achieved using different types of sensors, searching for less expensive ones that can perform rapid detection. Three different types of sensor are installed in Doris. The RFID System, the omnidirectional camera, and the laser range finder.

In this section, the method of obtaining data from the sensors, by detecting their respective landmarks and their location by either distance or angle from the robot, are 
presented.

\section{RFID detection}

With regard to pose estimation using RFID, the most common method is based on triangulation with the received signal strength indicator (RSSI) as explained in [80], due to its low cost and ubiquity, although those advantages do not imply that it is the best approach to locate objects. RSSI measurements involve high uncertainty margins, according to [81] achieving a mean error from $2.9 \mathrm{~m}$ to $4.3 \mathrm{~m}$ on a floor area of $980 \mathrm{~m}^{2}$. The most notable contribution was made by [82] in which a tag position error of $1.08 \mathrm{~m}$ was achieved within a limited space.

Other application can be found in [83] which places each the RFID tag on the floor in a fixed and constant distance respect to each other creating a tag matrix and places the reader beneath the robot in order to detect each tag and this fixes the robot position in $X(k)$ and $Y(k)$ and the robot orientation is fixed by using a camera and detecting $\mathrm{QR}$ codes.

The applications on guiding robots using RFID systems in narrow areas (through hallways or negotiating a door) are really low, so a certain degree of precision is needed that is not provided by this type of system, but it can be useful for other localization tasks as global positioning.

The RFID Impinj R220 reader system installed in Doris has two right circular polarity antenna, from which three different measures can be obtained:

1. RSSI: Received Signal Strength Indicator, which provides an estimate of the tag backscatter signal power received $P_{R}$ in Watts with an RFID reader [84] by using the Friis equation:

$$
P_{R}=\frac{G_{T}^{2} * \lambda^{2} * \sigma}{(4 * \pi)^{3} * R^{4}} * P_{T}
$$

Where:

$P_{T}$ is the reader transmitter power at the transmit antenna input (Watts).

$G_{T}$ is the reader antenna gain.

$\lambda$ is the carrier wavelength (m), which can be estimated as: $\lambda=\frac{c}{f}$ where, $c$ is the speed of the electromagnetic wave and $f$ is the operational frequency of the antenna.

$\sigma$ is the Tag Radar Cross Section $\left(m^{2}\right)$, computed as $\sigma=\frac{G_{R} * \lambda^{2}}{4 * \pi}$, where $G_{R}$ is the antenna receiver gain.

$R$ is the distance between reader and tag $(\mathrm{m})$.

2. RF phase angle: represents the phase rotation angle over distance [84], which the 
reader obtains by using the following formula:

$$
\theta=2 * \pi *\left(\frac{2 * R}{\lambda}\right)+\theta_{T}+\theta_{R}+\theta_{T A G}
$$

where, $\theta_{T}, \theta_{R}, \theta_{T A G}$ are the phase angle rotations of the transmitter circuit, the receiver circuit, and the tag reflection characteristic, respectively.

As the phase is a periodic function with a period of $2 * \pi$ radians, the values will repeat at distances separated by multipliers of half a wavelength: $R_{n}=\frac{n * \lambda}{2}$ where, $n=0,1,2, \ldots$

3. Doppler Frequency: is the shift of the received signal at the reader, due to relative motion between the reader and the tag. This feature is helpful for determining the tags that are in motion. This feature will be used for future applications.

Both, RSSI and RF Phase provide information on the distance by computing the equation based on the information from the reader obtained from both 4.1 and 4.2 :

$$
R=\sqrt[4]{\frac{G_{T}^{2} * \lambda^{2} * \sigma}{(4 * \pi)^{3} * P_{R}} * P_{T}}
$$

where, the antenna operates at a frequency of $865.9 \mathrm{MHz}$, resulting in a wavelength of $\lambda=0.3464 m$ and the transmitter and receiver gain are also fixed values.

$$
R=\left(\theta-\theta_{T T}\right) * \frac{\lambda}{4 * \pi}
$$

where, $\theta_{T T}$ is the total phase rotation of $\theta_{T}+\theta_{R}+\theta_{T A G}$ to be determined in section 4.1 .2

The Ultra Wide-Band range measurement (UWB) is a radio technology similar to the RFID system which operates at higher frequencies. Nevertheless, it was not considered during the selection process due to the high price it has when compared to the RFID System. Although it is well known that it has a good accuracy for tags position estimation and this could be useful for localization of the robots, some issues must be considered:

1. Three anchors (antennas) minimum in each room are needed to achieve a good tag localization.

2. As stated, Doris is intended to work in a complex environment like a museum as a tour-guide, and it will be very difficult to install UWB anchors at these places.

3. Supposing that museums are in the will to install the anchors, another problem may araise because the radio frequency wave of the anchors can penetrate thin walls and some museum have very thick walls and depending on the area of the room, extra anchors might be needed. 
4. Costs increase when more anchors are added and a whole UWB system would be very expensive. Table 4.1 shows the costs of installing an UWB network.

Table 4.1: OpenRTLS UWB price

\begin{tabular}{cccc}
\hline Quantity & Description & Price Unit $(€)$ & Subtotal $(€)$ \\
\hline 1 & Master anchor & 220 & 220 \\
10 & Anchors & 220 & 2200 \\
1 & Tag & 65 & 65 \\
1 & SDK & 100 & 100 \\
1 & Switch & 80 & 80 \\
100 & Wiring & 3 & 300 \\
& & Total: & 2965 \\
\hline
\end{tabular}

This table shows the price of an UWB network provided by OpenRTLS and using 11 anchors in an area of $10 \mathrm{~m} x 10 \mathrm{~m}$, with the purpose to achieve a precise localization of the robot. Compared to the RFID system where antennas can be installed on the robot and tags can be sticked to the walls, the cost of the UWB System is a lot higher.

\section{Visual detection}

Visual detection has been used in mobile robots like used in [85] and [86] which uses a set of QR codes creating a matrix and covering the entire floor to achieve the robot orientation and [87] which is similar to the previous but the camera points to the ceiling instead. The sensor installed on the robot platform is an omnidirectional camera and the fiducial markers are placed on the walls with certain distribution along the room where the robot is going to perform. The reason for installing this type of camera is to provide Doris with a 360 degree field of view, so it can see the whole room and can pick up markers from all directions; for example, in a museum with halls of different sizes. The model used is the Mobotix C25 hemispheric camera, as shown in figure 4.1. This is an indoor ceiling camera with a diameter of $12 \mathrm{~cm}$ and a weight of $200 \mathrm{~g}$. It includes a light-sensitive $6 \mathrm{MP}$ day and night sensor and is powered and communicates via the ethernet.

The camera offers different types of display modes of different quality and resolution. In this case, only the full view is used at a resolution of 1280x960 pixels. 


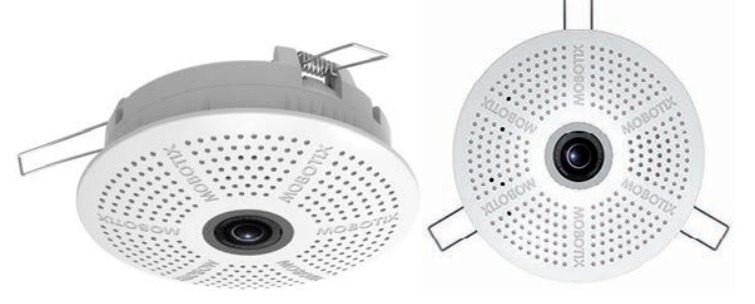

Figure 4.1: Omnidirectional camera C25 by Mobotix

The main goal is to recognize and to estimate the angle of the visual markers. These markers are a QR binary code containing information represented in a $5 x 5$ matrix. This information contains the landmark orientation as well as the map, sector and landmark Id's . The appearance of the marker is in figure 4.2, printed over an A3 paper size This code is robust against rotation and perspective views. The markers are located over the wall at $2.5 \mathrm{~m}$ above ground level, considering the height of the robot that is $\pm 1.45 \mathrm{~m}$.

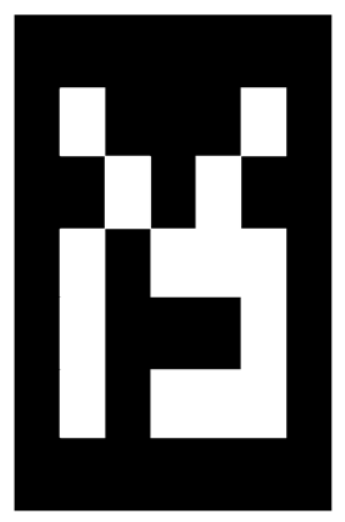

Figure 4.2: Example of a visual landmark

So the problem of locating the robot by using visual landmarks in large areas concerns only the determination of its angle from the center of the camera lens to the center of the marker. This problem can be solved by using OpenCV library, following the steps listed below:

1. Capture a frame from the scene, as shown in figure $4.3 \mathrm{a}$

2. The captured frame is converted to grayscale, as in figure $4.3 \mathrm{~b}$.

3. Image binarization. Due to different light conditions, the adaptive threshold is chosen for a fixed image so that it is homogenous, which requires two different values: a pixel size, which is invariant between images, and an index obtained by analyzing the histogram with which the mean intensity value is set. 
4. Contour detection. The binary image is submitted to find all available contours by retrieving a vector of points. This contour is stored in another vector of contours, only if the contour size is higher or equal than a predefined number of points, which in this case is set at 50 points. This value is used for contours that might be too small to be studied and are considered as noise. Figure $4.3 \mathrm{~d}$ shows the contours detected in the scene.

5. Possible marker detection. Consisting of selecting only those contours which are a convex polygon with only 4 sides and discarding everything else. At this point, a list of possible markers is created and refined by keeping only those that match a square or a rectangle.

6. Real marker selection and decoding. As the algorithm must be robust for rotations and transformations of the possible marker that is detected, the perspective transform must be calculated for each one. A transformation that is done by using four pairs of the corresponding points in a real scene. Once the perspective transformational matrix is estimated, a perspective transformation to the image is applied, and the resulting image is sent for decoding.

As the image contains only black and white cells of a known size, the decoding first consists of building a bitmap based on the information obtained by the image. As it is a binary image, the non-zero counting for each cell is performed to establish every bit, and by applying the Hamming distance, the marker can be recognized and the associated information can be obtained. After the perspective correction, the image can be in 4 positions and the Hamming distance of each one is tested. The zero distance will be chosen as the winning rotation, obtaining a code from the marker, and searching for it in a database that yields a reference for global positioning.

7. Angle estimation. Considering that the camera offers a circular (somewhat similar to fish-eye) image, the angle estimation is done through only one calculation:

$$
\phi=\tan ^{-1}\left(\frac{Y_{\text {ImageCenter }}-Y_{\text {MarkerCenter }}}{X_{\text {ImageCenter }}-X_{\text {MarkerCenter }}}\right)-\frac{\pi}{2}
$$

As the figure $4.3 \mathrm{e}$ shows, after applying the previous steps, the markers are detected. As the camera is connected to other computer where the image processing is executed, processing 20 frames per seconds. Then the data is transmitted to the onboard computer every $10 \mathrm{~ms}$ via UDP socket.

\section{Reflective landmarks detection}

Laser technology is widely used in robotics, especially for map design using the SLAM algorithm. It is only used for landmark detection in this study with some specifications. 


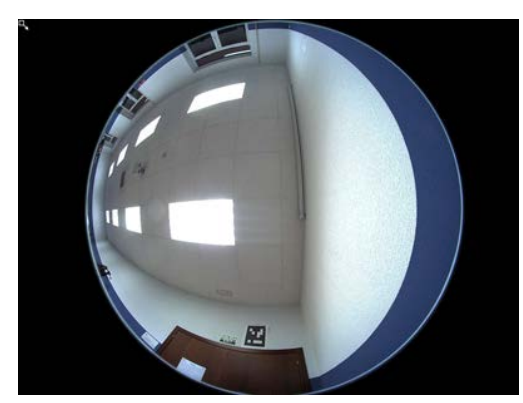

(a) Full color scene

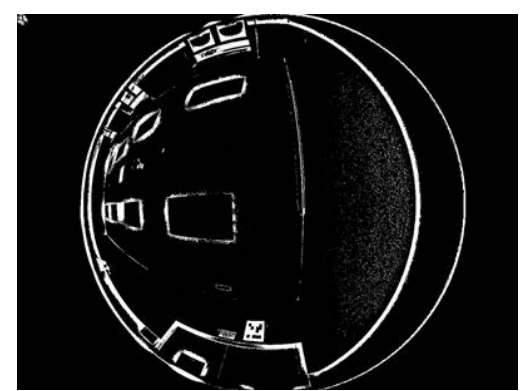

(c) Grayscale thresholded according to environment

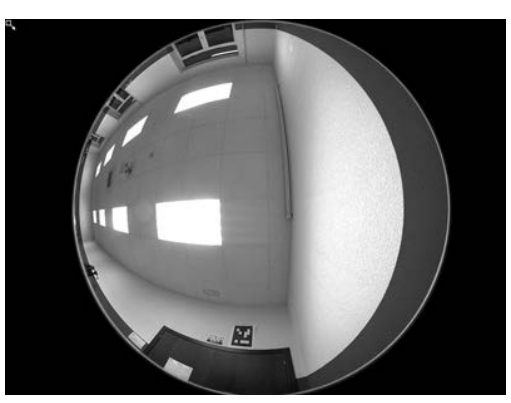

(b) Captured scene converted to grayscale

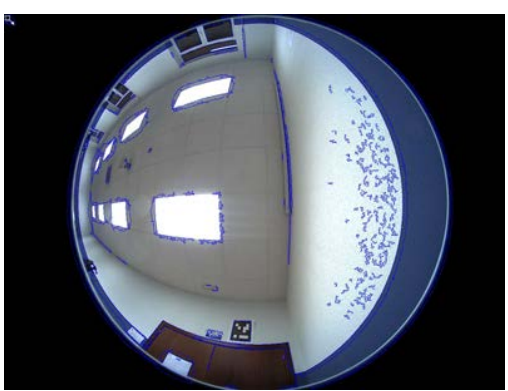

(d) Contours detected matching criteria

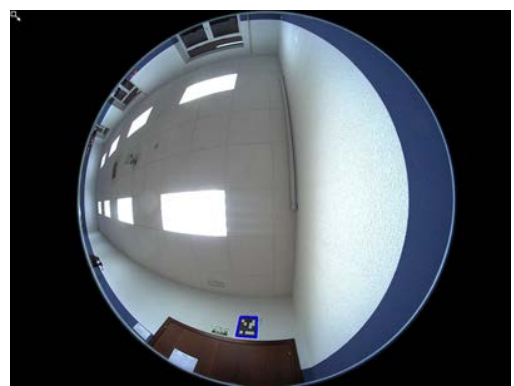

(e) Markers detected

Figure 4.3: Images obtained in the treatment process

These landmarks have a circular shaped reflective cover and were designed ad hoc.

One function of the laser is to add certain properties to each measurement; for example, the reflection intensity when the beam strikes a reflective surface. Taking advantage of this property, each landmark was provided with its respective reflective material.

The reason for their circular shape is because a planar landmark with the same reflective material in earlier tests could only be detected by the laser when the beam struck right in front of the landmark, otherwise, the beam would bounce to another direction and would not return to the laser. Figure 4.4 shows the prototype of the 
landmark design. Landmarks are designed to be positioned next to walls, hence their limitation to only 180 degrees.
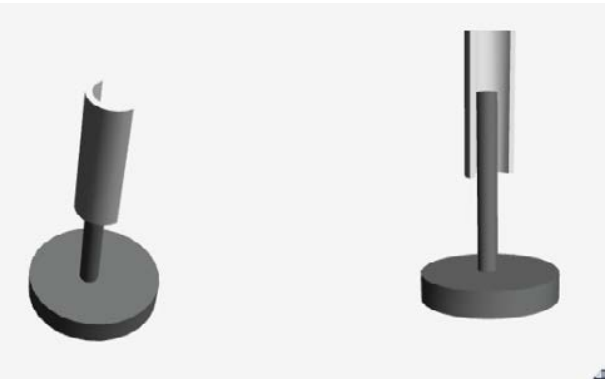

Figure 4.4: Reflective landmark designed by the group

It is assumed that the center of the reflective landmark may be located with maximum precision where it is a perfect cylinder of a known radius $(r=0.045 m)$. The SICK laser adds a reflective intensity to each of the 361 measurements and identifies the landmarks. More than one intensity can belong to the same landmark. Hence, a classification condition must be implemented, in order to obtain the number of landmarks detected by the laser. First, only those measurements are selected that are of a higher intensity than zero. The condition for classification with the resulting vector of indices is as follows:

$$
I I[i]-I I[i+1]<\text { Threshold }
$$

where, $I I$ is the intensity index, meaning that the difference between two consecutive indices is lower than the threshold it will classify as the same landmark. Once the set of points associated for each landmark is obtained, the aim is then to obtain the landmark center. This point can be estimated by applying the Law of Sines and an iterative minimum squared error estimation for the error correction. So the aforementioned Law of Sines is used as an observation model.

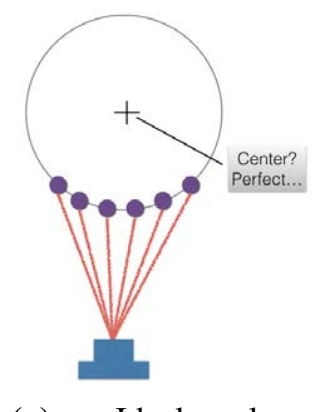

(a) Ideal laser behaviour

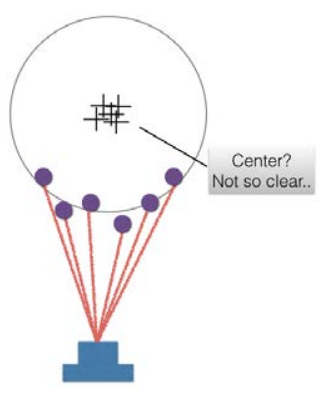

(b) Real laser behaviour

Figure 4.5: Measurement representation obtained by the laser

Each measurement obtained by the laser contains the distance and the angle to the 
point represented as $[\rho(k) \phi(k)]^{T}$, and a set of these consecutive points represents a single landmark, so that for the $i$-th landmark, there are $n$ points that represent its circumference, where $n \geq 2$.

$$
\left[\begin{array}{c}
\hat{\rho}_{1}^{i}(k) \\
\phi_{1}^{i}(k) \\
\\
\hat{\rho}_{2}^{i}(k) \\
\phi_{2}^{i}(k) \\
\vdots \\
\hat{\rho}_{n}^{i}(k) \\
\phi_{n}^{i}(k)
\end{array}\right]
$$

and the resulting mean with the radius added to the distance, for each landmark is expressed as:

$$
\left[\begin{array}{c}
\tilde{\rho}^{i}(k)+r \\
\tilde{\phi}^{i}(k)
\end{array}\right]
$$

The Law of Sines is used as the observational model for the iterative minimum squared error estimation that is expressed as:

$$
\frac{r}{\sin \left(\tilde{\phi}^{i}(k)-\phi_{j}^{i}(k)\right)}=\frac{\hat{\rho}_{j}^{i}(k)}{\sin (\alpha)}=\frac{\left(\tilde{\rho}^{i}(k)+r\right)}{\sin \left(\pi-\alpha-\tilde{\phi}^{i}(k)+\phi_{j}^{i}(k)\right)}
$$

Therefore, the distance estimation measured by the laser, for each $j$-th measure, is defined by:

$$
\begin{gathered}
h\left(j, \rho^{i}(k), \phi^{i}(k)\right)=\hat{\rho}_{j}^{i}(k)=\tilde{\rho}^{i}(k) \cdot \cos \left(\tilde{\phi}^{i}(k)-\phi_{j}^{i}(k)\right) \\
-r \cdot \cos \left(\sin ^{-1}\left(\frac{\tilde{\rho}^{i}(k)}{r} \cdot \sin \left(\tilde{\phi}^{i}(k)-\phi_{j}^{i}(k)\right)\right)\right)
\end{gathered}
$$

Figure 4.6 explains the notation of equation 4.10, showing the correspondence of each variable involved. So, the value $\rho_{j}^{i}(k)$ given by laser should be the sum of the estimation plus a random error $w(k)$, which will be considered with zero mean and $R(k)$ as variance, which reduces the observation model to:

$$
\rho_{j}^{i}(k)=\hat{\rho}_{j}^{i}(k)+w(k)=h\left(j, \rho^{i}(k), \phi^{i}(k)\right)+w(k)
$$




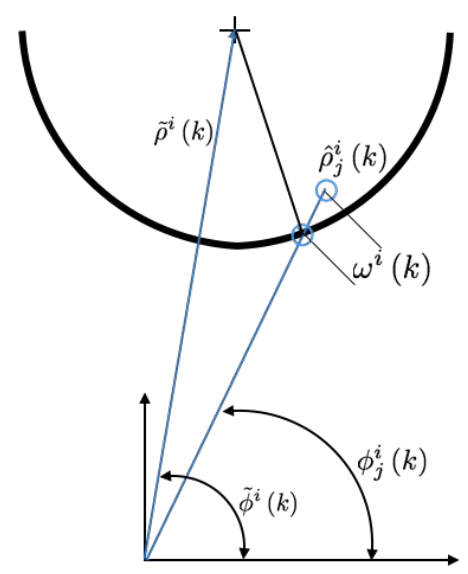

Figure 4.6: Estimation model for landmark center

Using equation 4.10, the Jacobian matrix is computed as shown in equations 4.12 , 4.13 and 4.14

$$
\begin{gathered}
\mathbf{H}^{i}(k)=\left[\begin{array}{ll}
\frac{\partial h}{\partial \rho} & \frac{\partial h}{\partial \phi}
\end{array}\right] \\
\frac{\partial h}{\partial \rho}=\cos \left(\tilde{\phi}^{i}(k)-\phi_{j}^{i}(k)\right)+\tilde{\rho}^{i}(k) \cdot\left(\cos \left(\tilde{\phi}^{i}(k)-\phi_{j}^{i}(k)\right)\right)^{2} . \\
r^{-1} \frac{1}{\sqrt{1-\frac{\tilde{\rho}^{i}(k) \cdot\left(\cos \left(\tilde{\phi}^{i}(k)-\phi_{j}^{i}(k)\right)\right)^{2}}{r^{2}}}} \\
\frac{\partial h}{\partial \phi}=-\tilde{\rho}^{i}(k) \cdot \sin \left(\tilde{\phi}^{i}(k)-\phi_{j}^{i}(k)\right)+\left(\tilde{\rho}^{i}\right)^{2}(k) \cdot \sin \left(\tilde{\phi}^{i}(k)\right. \\
\left.-\phi_{j}^{i}(k)\right) \cos \left(\tilde{\phi}^{i}(k)-\phi_{j}^{i}(k)\right) \\
\cdot r^{-1} \frac{1}{\sqrt{1-\frac{\tilde{\rho}^{i}(k) \cdot\left(\cos \left(\tilde{\phi}^{i}(k)-\phi_{j}^{i}(k)\right)\right)^{2}}{r^{2}}}}
\end{gathered}
$$

By applying these equations to each $j$-th of the $n$ measurement obtained by the laser, a least square method is applied for final correction. Two matrices are used to the achieve a good solution, one of them is the $\mathbf{P}^{i}(k)$, which indicates the truthfulness of the estimation and a gain matrix $\mathbf{W}^{i}(k)$, which is a estimated by using the laser covariance $\mathbf{R}(k)$, the position covariance $\mathbf{P}^{i}(k)$, and the $\mathbf{H}^{i}(k)$.

As previously mentioned, the laser error must be inserted, to build the gain Matrix $\mathbf{W}^{i}(k)$, which generates a diagonal matrix $\mathbf{R}(k)_{n \times n}$ with the typical deviation given by 
the laser provider: equal to $0.003^{2}$.

$$
\mathbf{R}(k)=\left[\begin{array}{cccc}
0.003^{2} & 0 & \cdots & 0 \\
0 & 0.003^{2} & \ldots & 0 \\
\vdots & \ddots & \cdots & \vdots \\
0 & \cdots & 0 & 0.003^{2}
\end{array}\right]
$$

This result yields a covariance matrix of the observations that is estimated as:

$$
\mathbf{S}^{i}(k)=\mathbf{H}^{i}(k) \cdot \mathbf{P}^{i}(k) \cdot \mathbf{H}^{i}(k)^{T}+\mathbf{R}(k)
$$

Having the following gain matrix:

$$
\mathbf{W}^{i}(k)=\mathbf{P}^{i}(k) \cdot \mathbf{H}^{i}(k)^{T} \cdot \mathbf{S}^{i}(k)^{-1}
$$

And naming innovation array $\boldsymbol{\nu}^{i}(k)$, as the difference between measurements obtained by the laser and those obtained by the observation model:

$$
\boldsymbol{\nu}^{i}(k)=\left[\begin{array}{c}
\rho_{1}^{i}(k) \\
\rho_{2}^{i}(k) \\
\vdots \\
\rho_{n}^{i}(k)
\end{array}\right]-\left[\begin{array}{c}
\hat{\rho}_{1}^{i}(k) \\
\hat{\rho}_{2}^{i}(k) \\
\vdots \\
\hat{\rho}_{n}^{i}(k)
\end{array}\right]
$$

The final correction is expressed by:

$$
\left[\begin{array}{c}
\tilde{\rho}^{i}(k) \\
\tilde{\phi}^{i}(k)
\end{array}\right]=\left[\begin{array}{c}
\tilde{\rho}^{i}(k) \\
\tilde{\phi}^{i}(k)
\end{array}\right]+\mathbf{W}^{i}(k) \cdot \boldsymbol{\nu}^{i}(k)
$$

This procedure allows to obtain up to 6 landmarks each $10 \mathrm{~ms}$.

\subsubsection{Sensors error estimation}

The measurement error must first be computed, before starting to work with the inner odometry of the robot, the RFIDs, the laser, and the camera. This error, present in every single measurement, is denoted as white noise, and is time variant. The aim of these experiments is to achieve a Gaussian distribution from all sensors for each variable, both in distance and in angle; in other words, the uncertainty of each measurement will be computed.

\section{Odometry standard deviation}

This error term needs to be estimated, in order to be represented as a covariance matrix, where its principal diagonal are the variances of the error in distance and yaw angle (heading, $\theta$ ) of the robot.

$$
Q(k)=\left(\begin{array}{cc}
\sigma_{d r}^{2} & 0 \\
0 & \sigma_{\theta r}^{2}
\end{array}\right)
$$


Doris was intended to move forward and backwards in any direction and to spin in circles, to obtain the values of $\sigma_{d r}^{2}$ and $\sigma_{\theta r}^{2}$. Every position reported by the robotic encoders was registered in a file and the difference between the position provided by the encoders versus the distance and angle measured with external sensors is taken as the error. When the robot is moving forward or backwards it tends to lean to the right, due to wheel pressure differences, adding a bias. One thing to be considered is that the robot receives linear and angular speed commands as inputs and the model in use is referenced in drive and steer increments as inputs, so the robots always moves at the same speed. The results are shown in the table below:

Table 4.2: Process Noise standard deviation

\begin{tabular}{cc}
\hline Distance (m) & Angle (rad) \\
\hline 0.029444 & 0.04019 \\
\hline
\end{tabular}

$$
Q(k)=\left(\begin{array}{cc}
0.000867 & -0.00049 \\
-0.00049 & 0.001616
\end{array}\right)
$$

\section{Laser standard deviation}

In section 4.1.1, the operation of the laser range device was briefly explained. However, its behavior tends to add uncertainty to the measurement, for which reason a procedure was followed to obtain the mean and variance values for distance and angular uncertainty.

1. The robot was placed in an area where the landmarks can be freely moved.

2. The area is approximately $1.6 \mathrm{~m}$ wide and $4.0 \mathrm{~m}$ heigh and was divided into small areas of $0.16 m^{2}$

3. A measurement was taken in four different orientations, in order to estimate the distance and angle and to compare them with the real measurements

4. This procedure was repeated 25 times for each orientation.

A sample iteration is shown in the following table: 
Table 4.3: Reflective landmark real position vs estimated position

\begin{tabular}{cccc}
\hline $\begin{array}{c}\text { Real } \\
\text { distance (m) }\end{array}$ & $\begin{array}{c}\text { Real } \\
\text { angle (rad) }\end{array}$ & $\begin{array}{c}\text { Estimated } \\
\text { distance (m) }\end{array}$ & $\begin{array}{c}\text { Estimated } \\
\text { angle (rad) }\end{array}$ \\
\hline 0.8944 & 0.4636 & 0.8957 & 0.4916 \\
0.8000 & 0.0000 & 0.7948 & -0.0038 \\
0.8944 & -0.4636 & 0.8844 & -0.4886 \\
1.2649 & 0.3217 & 1.2656 & 0.3272 \\
1.2000 & 0.0000 & 1.1872 & 0.0002 \\
\hline
\end{tabular}

where, columns 1 and 2 are the real distance and angle from the sensor to the landmark, columns 3 and 4 are the estimated distance and angle provided by the laser. In table 4.4 columns 1 and 2 are the error between the real and the estimated values. In figure 4.7 can be seen the previous table. This experiment was performed up to $5 \mathrm{~m}$ in $\mathrm{x}$-coordinate and $5 \mathrm{~m}$ in y-coordinate, but only few results are shown in the tables and the respective figure.

Table 4.4: Reflective landmark errors at different distances

\begin{tabular}{cc}
\hline $\begin{array}{c}\text { Error } \\
\text { distance }(\mathbf{m})\end{array}$ & $\begin{array}{c}\text { Error } \\
\text { angle (rad) }\end{array}$ \\
\hline-0.0013 & -0.0279 \\
0.0051 & 0.0038 \\
0.0099 & 0.0250 \\
-0.0007 & -0.0054 \\
0.0127 & -0.0002 \\
\hline
\end{tabular}

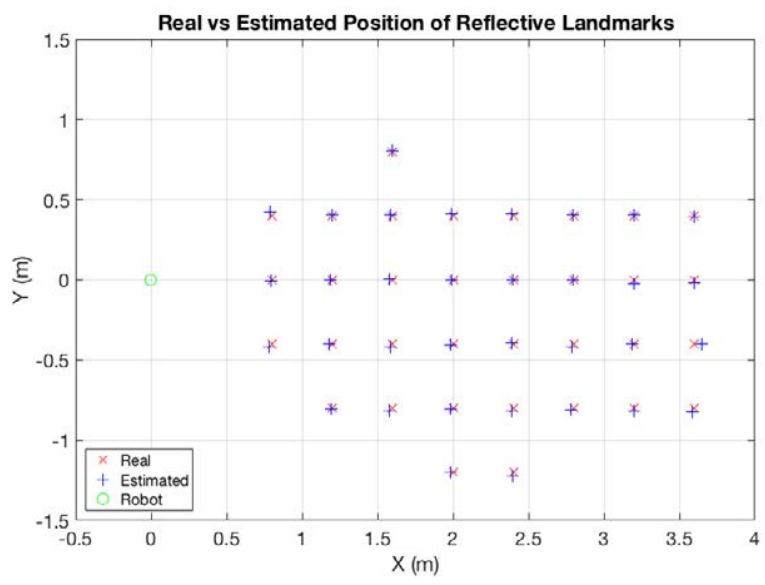

Figure 4.7: Real vs estimated position of reflective landmarks

Where the green 'o' is the location of the robot, the red ' $x$ ' represent the real position 
of the landmark (columns 1 and 2 of table 4.3 ) and the blue ' + ' the position estimated and fixed by the detector explained in section 4.1.1 (columns 3 and 4 of table 4.3 ).

Table 4.5: Reflective landmark standard deviation

\begin{tabular}{cc}
\hline Distance (m) & Angle (rad) \\
\hline 0.00911 & 0.005196 \\
\hline
\end{tabular}

as can be seen in table 4.4, the error is a random value. The standard deviations for the resultant distance and angle are shown in table 4.5 .

$$
R(k+1)=\left(\begin{array}{cc}
0.000083 & 0 \\
0 & 0.000027
\end{array}\right)
$$

The covariance between laser distance and angle is around $10^{-7}$, which is practically negligible.

\section{Camera standard deviation}

Using a similar procedure as in 4.1.2, a marker that is $2.5 \mathrm{~m}$ above ground level (shown in figure 4.8 was placed at different positions and distances from the robot. The error of different angles from the camera at different distances is shown in the following table.

Table 4.6: Angle error at different distances

\begin{tabular}{ccccc}
\hline Distance $(\mathbf{c m})$ & 0 $(\mathbf{r a d})$ & $\frac{\pi}{2}(\mathbf{r a d})$ & $\pi(\mathbf{r a d})$ & $\frac{3 \pi}{2}(\mathbf{r a d})$ \\
\hline 20 & 0.06088 & & & \\
40 & 0.06776 & 0.03297 & & \\
60 & 0.05457 & 0.03258 & 0.07663 & 0.13908 \\
80 & 0.05994 & 0.03031 & 0.07577 & 0.10758 \\
100 & 0.0595 & 0.02915 & 0.05832 & 0.09835 \\
120 & 0.06258 & 0.03183 & 0.07348 & 0.07687 \\
140 & 0.06045 & 0.03431 & 0.06854 & 0.08102 \\
160 & 0.06138 & 0.04255 & 0.05873 & 0.07578 \\
180 & 0.06005 & 0.04744 & 0.06511 & 0.06926 \\
200 & 0.05909 & 0.04658 & 0.06011 & 0.06911 \\
\hline
\end{tabular}

Table 4.7: Angle Standard Deviation

\begin{tabular}{cccc}
\hline 0 $(\mathbf{r a d})$ & $\frac{\pi}{2}(\mathbf{r a d})$ & $\pi(\mathbf{r a d})$ & $\frac{3 \pi}{2}(\mathbf{r a d})$ \\
\hline 0.00339 & 0.00954 & 0.00740 & 0.02122 \\
\hline
\end{tabular}




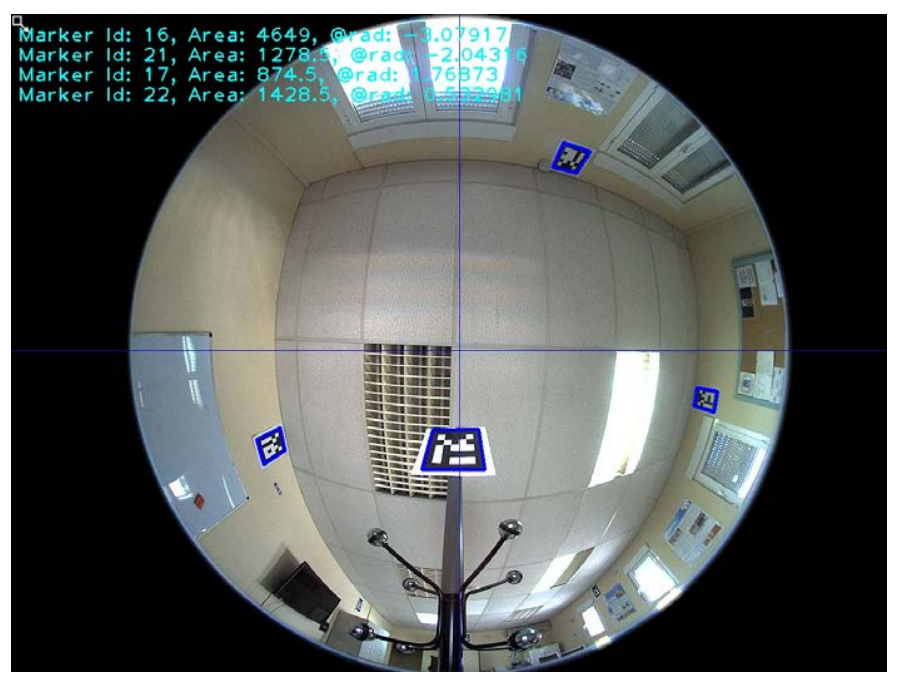

Figure 4.8: Visual marker placed at zero degrees

As can be seen in table 4.6, the error is higher when the landmark is closer to the camera, which makes sense because the closest marker to the camera has more pixel variations. The standard deviations for each angle are shown in table 4.7 where the highest deviation for the EKF filter is selected by the following equation:

$$
R(k+1)=\left(0.00045 \mathrm{rad}^{2}\right)
$$

\section{RFID standard deviation}

The RFID system can be powerful for many applications, such as identifying people, logistic and supply chain visibility, race timing, access control, among others, yet it is not very powerful for the purposes of localization. Designing a Real-Time Location System (RTLS) using RFIDs is no easy job. Environment and other effects assume an important place when the reader estimates RSSI and phase angles. Propagation effects such as absorption and scattering can reduce the power observed at the reader receiver, as well as multipath propagation and undesired signals in the environment, as mobile networks and Wi-Fi can combine with primary backscatter, increasing or decreasing the RSSI value.

Phase angle is estimated by using the RSSI as a function of the Signal to Noise Ratio (SNR), so the more noise energy within the receiver bandwidth, the greater the phase standard deviation. In addition, the reader receives signal processing that introduces $\pi$ radian ambiguity, so that the reported phase can be the true phase $(\theta)$ or the true phase plus $\pi$ radians $(\theta+\pi)$.

According to [84], the standard deviation of the RSSI System is: 
Table 4.8: Standard deviation of RSSI and RF Phase

\begin{tabular}{cccc}
\hline Description & Min & Max & Unit \\
\hline RSSI & & 1 & $\mathrm{~dB}$ \\
RF Phase & -0.1 & 0.1 & $\mathrm{rads}$ \\
\hline
\end{tabular}

These values were obtained from mean value over 1000 tags in an anechoic chamber. However, the robot will not move around in anechoic spaces. The typical surroundings will be, for example, in a museum where several people will interact with Doris at different times. Moreover, objects from a museum like sculptures will represent obstacles for the robot, affecting every measurement taken by the RFID system. The following table shows how this system works in an indoor environment where the robot will perform, calculating the distance from antenna to tag by using equation (4.1). Due phase is a periodical function and given the great difficulty in determining the distance from tag to reader by using only one phase, the phase equation method was discarded:

Table 4.9: Values of RSSI and distance in a complex environment

\begin{tabular}{ccccc}
\hline $\begin{array}{c}\text { Real } \\
\text { distance }(\mathbf{m})\end{array}$ & $\begin{array}{c}\text { RSSI } \\
\text { 0 rad }(\mathbf{d B})\end{array}$ & $\begin{array}{c}\text { Distance } \\
\text { 0 rad }(\mathbf{m})\end{array}$ & $\begin{array}{c}\text { RSSI } \\
\frac{\pi}{4} \mathrm{rad}(\mathrm{dB})\end{array}$ & $\begin{array}{c}\text { Distance } \\
\frac{\pi}{4} \mathrm{rad}(\mathrm{m})\end{array}$ \\
\hline 1.00 & -37 & 0.929964 & -47.5 & 1.702025 \\
1.50 & -46 & 1.561227 & -53.5 & 2.404175 \\
2.00 & -40 & 1.965468 & -59.5 & 3.395987 \\
2.50 & -43 & 1.351967 & -53 & 2.335963 \\
3.00 & -56 & 2.776298 & -64 & 4.400135 \\
3.50 & -56 & 2.776298 & -61 & 3.702253 \\
4.00 & -63 & 4.275295 & -57 & 2.940804 \\
\hline
\end{tabular}

Table 4.9 shows the real distance from the antenna to the tag in the first column, the reported RSSI with the tag in front of the antenna in the second column, where the phase is around 0rads, and the RSSI value converted to distance in the third column. Columns 4 and 5 are the same as the two previous ones, but with the tag placed at 45 degrees from the antenna. The results show that if the tag is placed right in front of the antenna (Line of Sight), then the distance will be more feasible, but with a small deviation from the center, the reader will report a higher RSSI and the distance will be erroneous.

Table 4.10: RFID standard deviation

\begin{tabular}{cc}
\hline Distance error @ 0 rad(m) & Distance error @ $\frac{\pi}{4} \mathbf{r a d}(\mathbf{d B})$ \\
\hline 0.496876 & 0.892177 \\
\hline
\end{tabular}

As can be seen in previous table, the error is too high, added to which the EKF will lead to undesired results and the phase will be useless, because it will depend on the RSSI 
value. Hence, the RFID system will not be used for the localization system and will be used only for navigation purposes that will be detailed in section 4.4 .

\subsubsection{Kalman filter and integration}

Each sensor described above appears to work quite well alone, but one of the main purposes of this research is to ensure that they all work in synergy with the odometry of the robot by means of sensor fusion. One way to achieve this aim is by using the Kalman Filter. As stated, the position of the state vector of the robot is defined as:

$$
\mathbf{x}(k)=\left[\begin{array}{c}
x(k) \\
y(k) \\
\theta(k)
\end{array}\right]
$$

The main idea is to minimize the odometrical error that can be caused by cumulative errors in encoders, slippery wheels, etc. An adaptation of the Kalman Filter can achieve this goal.

\section{Kalman filter}

Proposed by [88], the filter consists of a set of mathematical equations to arrive at an effective solution for the least squares problem, making it robust and powerful, due to the fact that it is based on past, present, and future data. It is summarized in the form of a recursive least square algorithm for dynamic systems.

The algorithm approaches the problem for state estimation of a discrete lineal process through data sets which can have noisy values. Based on this filter, other versions for nonlinear systems are the Unscented Kalman Filter (UKF) and the Extended Kalman Filter (EKF). The latter has been chosen to be used in this research due to the computational cost of the UKF. Although it is better for strong nonlinearities, previous works in robot localization showed similar results in practice [89, 90]. Regarding the use of the EKF instead of a particle filter, the main reason for this choice is that the designed landmarks are highly distinctive and data association errors are not very likely.

The first step for the EKF is the prediction model:

$$
\begin{gathered}
\mathbf{x}(k+1 \mid k)=\mathbf{f}(k, \mathbf{x}(k \mid k), \mathbf{u}(k)+\mathbf{v}(k)) \\
\tilde{\mathbf{x}}(k+1 \mid k)=\mathbf{f}(k, \tilde{\mathbf{x}}(k \mid k), \mathbf{u}(k))
\end{gathered}
$$

Based on this equation, Doris uses the following values for the prediction: 


$$
\left[\begin{array}{c}
\tilde{x}(k+1 \mid k) \\
\tilde{y}(k+1 \mid k) \\
\tilde{\theta}(k+1 \mid k)
\end{array}\right]=\left[\begin{array}{c}
\tilde{x}(k \mid k) \\
\tilde{y}(k \mid k) \\
\tilde{\theta}(k \mid k)
\end{array}\right]+\left[\begin{array}{c}
\Delta \rho(k) \cos \left(\tilde{\theta}(k \mid k)+\frac{\Delta \phi(k)}{2}\right) \\
\Delta \rho(k) \sin \left(\tilde{\theta}(k \mid k)+\frac{\Delta \phi(k)}{2}\right) \\
\Delta \phi(k)
\end{array}\right]
$$

where, $[\Delta \rho(k) \Delta \phi(k)]^{T}$ is the system input defined as the drive $\Delta \rho(k)$ and the incremental values of the steering encoders $\Delta \phi(k)$. And for upgrading the position uncertainty, the following is used:

$$
\mathbf{P}(k+1 \mid k)=\mathbf{A}(k) \mathbf{P}(k \mid k) \mathbf{A}^{T}(k)+\mathbf{G}(k) \mathbf{Q}(k) \mathbf{G}^{T}(k)
$$

where, $\mathbf{P}(k \mid k)$ is the current state covariance matrix and $\mathbf{Q}(k)$ is the process covariance matrix. $\mathbf{A}(k)$ and $\mathbf{G}(k)$ are the Jacobian matrices that are computed as:

$$
\mathbf{A}(k)=\nabla \tilde{\mathbf{f}}_{\tilde{\mathbf{x}}(k \mid k)} ; \quad \mathbf{G}(k)=\nabla \tilde{\mathbf{f}}_{\tilde{\mathbf{u}}(k)}
$$

yielding:

$$
\begin{gathered}
\mathbf{A}(k)=\left[\begin{array}{ccc}
1 & 0 & -\Delta \rho(k) \sin \left(\theta(k \mid k)+\frac{\Delta \phi(k)}{2}\right) \\
0 & 1 & \Delta \rho(k) \cos \left(\theta(k \mid k)+\frac{\Delta \phi(k)}{2}\right) \\
0 & 0 & 1
\end{array}\right] \\
\mathbf{G}(k)=\left[\begin{array}{cc}
\cos \left(\theta(k \mid k)+\frac{\Delta \phi(k)}{2}\right) & -0.5 * \Delta \rho(k) \sin \left(\theta(k \mid k)+\frac{\Delta \phi(k)}{2}\right) \\
\sin \left(\theta(k \mid k)+\frac{\Delta \phi(k)}{2}\right) & 0.5 * \Delta \rho(k) \cos \left(\theta(k \mid k)+\frac{\Delta \phi(k)}{2}\right) \\
0 & 1
\end{array}\right]
\end{gathered}
$$

The prediction of the observations and their covariance matrix have to be computed:

$$
\begin{gathered}
\tilde{\mathbf{z}}(k+1)=\mathbf{h}(k+1, \tilde{\mathbf{x}}(k+1 \mid k)) \\
\mathbf{S}(k+1)=\mathbf{H}(k+1) \mathbf{P}(k+1 \mid k) \mathbf{H}^{T}(k+1)+\mathbf{R}(k+1)
\end{gathered}
$$

where, $\mathbf{R}(k+1)$ is the measurement covariance matrix, where every landmark is inputted with its Cartesian coordinates in a database:

$$
\mathbf{x}_{L}=\left[\begin{array}{l}
x_{L} \\
y_{L}
\end{array}\right]
$$

so the observation is the relative position of the landmark with respect to the robot, in other words, the model used in this case is:

$$
\tilde{\mathbf{z}}(k+1)=\left[\begin{array}{c}
\rho(k+1) \\
\phi(k+1)
\end{array}\right]=\left[\begin{array}{c}
\sqrt{\left(x_{L}-\tilde{x}(k+1 \mid k)\right)^{2}+\left(y_{L}-\tilde{y}(k+1 \mid k)\right)^{2}} \\
\tan ^{-1}\left(\frac{y_{L}-\tilde{y}(k+1 \mid k)}{x_{L}-\tilde{x}(k+1 \mid k)}\right)-\tilde{\theta}(k+1 \mid k)
\end{array}\right]
$$


the matrix $\mathbf{H}(k+1)$ is the Jacobian matrix computed as follows:

$$
\mathbf{H}(k+1)=\nabla \mathbf{h}_{\tilde{\mathbf{x}}(k+1 \mid k)}
$$

which is computed for each landmark in the database, resulting in:

$$
\mathbf{H}(k)=\left[\begin{array}{ccc}
-\frac{x_{L}-\tilde{x}(k+1 \mid k)}{\sqrt{\left(x_{L}-\tilde{x}(k+1 \mid k)\right)^{2}+\left(y_{L}-\tilde{y}(k+1 \mid k)\right)^{2}}} & -\frac{y_{L}-\tilde{y}(k+1 \mid k)}{\sqrt{\left(x_{L}-\tilde{x}(k+1 \mid k)\right)^{2}+\left(y_{L}-\tilde{y}(k+1 \mid k)\right)^{2}}} & 0 \\
\frac{y_{L}-\tilde{x}(k+1 \mid k)}{\left(x_{L}-\tilde{x}(k+1 \mid k)\right)^{2}+\left(y_{L}-\tilde{y}(k+1 \mid k)\right)^{2}} & -\frac{x^{2}}{\left(x_{L}-\tilde{x}(k+1 \mid k)\right)^{2}+\left(y_{L}-\tilde{y}(k+1 \mid k)\right)^{2}} & -1
\end{array}\right]
$$

And the $\mathbf{W}(k+1)$ which is the gain of the Kalman Filter, is computed as:

$$
\mathbf{W}(k+1)=\mathbf{P}(k+1 \mid k) \mathbf{H}^{T}(k+1) \mathbf{S}^{-1}(k+1)
$$

The next step is to obtain every observation from every sensor:

$$
\mathbf{z}(k+1)
$$

Then the matching between every observation is performed. For reflective landmarks, an Euclidean distance between the polar coordinates of each landmark stored in the database $\mathbf{p}_{1}^{i}=\left(\rho_{L}^{i}, \phi_{L}^{i}\right)$ and the observation $\mathbf{p}_{2}=\left(\rho_{z}, \phi_{z}\right)$ is computed by using:

$$
\mathbf{d}^{i}\left(\mathbf{p}_{1}, \mathbf{p}_{2}\right)=\sqrt{\rho_{L}^{2}+\rho_{z}^{2}-2 * \rho_{L} * \rho_{z} \cos \left(\phi_{L}-\phi_{z}\right)}
$$

Where a distances set is obtained and the smallest distance of that set is chosen as the matching landmark observation.

With regard to the visual markers, it is easier to perform the matching, because it is a QR code containing information. As it is a $5 x 5$ matrix, the first and the last row are used for orientation, and the 3 middle rows are used to identify the map ( $2^{\text {nd }}$ row), the sector ( $3^{\text {rd }}$ row), and the landmark ( $4^{\text {th }}$ row). The first two rows will be used in the future for general localization, not implemented in this research, but the other row of the visual marker will be used to perform the matching between the observation and the database.

Once the landmarks have been matched, the measurement innovation is obtained:

$$
\boldsymbol{\nu}(k+1)=\mathbf{z}(k+1)-\tilde{\mathbf{z}}(k+1)
$$

but this innovation can be deleted, if the landmark does not satisfy the Mahalanobis distance acceptance test, computed as:

$$
\mathbf{d}_{m}^{i}=\frac{\boldsymbol{\nu}(k+1)}{\sigma_{s}}
$$


where, $\sigma_{s}$ is the standard deviation of the sensor. If the $\mathbf{d}_{m}^{i}$ is lower than a maximum Mahalanobis distance, then the measurement innovation is accepted, otherwise it is rejected.

The final position corrected by the EKF is computed as:

$$
\tilde{\mathbf{x}}(k+1 \mid k+1)=\tilde{\mathbf{x}}(k+1 \mid k)+\mathbf{W}(k+1) \boldsymbol{\nu}(k+1)
$$

And the uncertainty $\mathbf{P}(k+1 \mid k+1)$ is calculated as:

$$
\mathbf{P}(k+1 \mid k+1)=[I-\mathbf{W}(k+1) \mathbf{H}(k+1)] \mathbf{P}(k+1 \mid k)
$$

\section{Integrating sensors}

At this point, all sensors must be fused into the EKF, in order for the odometrical system to achieve better positioning of the robot. This operation is achieved by modifying some equations in the filter where sensor fusion takes place. In one of them, (4.35), every observation must be computed and every landmark position must be read from the database and referenced to the robot position. This operation will result in a matrix $2 * r l+v m$, where $r l$ is the number of reflective landmarks and $v m$ is the number of visual markers in the room, which is the result of the information given by each sensor (distance and angle to the landmark by the laser and angle to the marker by the camera).

The sensor type is very important, as it will set the order in which each sensor is taken into account in the filter. The main reason is to set an order for all matrices. The established order is:

1. Laser Range Finder (rl).

2. Omnidirectional Camera (vm).

Example. Suppose the robot in a certain position has the two sensors enabled and the room where it is located has three reflective landmarks and two visual markers. The predicted position is:

$$
\tilde{\mathcal{X}}(k+1 \mid k)=\left[\begin{array}{l}
5.2152 \\
2.3489 \\
3.1054
\end{array}\right]
$$


and the observation matrix $\tilde{\mathbf{z}}(k+1)$ will be:

$$
\tilde{\mathbf{z}}(k+1)=\left[\begin{array}{c}
\rho_{r l 1} \\
\phi_{r l 1} \\
\rho_{r l 2} \\
\phi_{r l 2} \\
\rho_{r l 3} \\
\phi_{r l 3} \\
\hline \phi_{v m 1} \\
\phi_{v m 2}
\end{array}\right]=\left[\begin{array}{c}
2.9530 \\
0.6492 \\
1.5731 \\
0.7242 \\
0.8512 \\
-1.5767 \\
\hline 0.0793 \\
-1.0485
\end{array}\right]
$$

The camera is not located at the exact center for the robot, so the deviation must be added to the observation, for it to be correctly computed.

Once the order in the matrices is defined, the other equation which is affected by the sensor fusion is 4.33 ; the $\mathbf{R}(k+1)$ is a square diagonal matrix, with the covariance values of every sensor that is enabled. This will result in the following covariance matrix:

$$
\left[\begin{array}{cccccc|cc}
\multicolumn{8}{c}{\mathbf{R}(k+1)=} \\
\sigma_{\rho r l}^{2} & 0 & 0 & 0 & 0 & 0 & 0 & 0 \\
0 & \sigma_{\phi r l}^{2} & 0 & 0 & 0 & 0 & 0 & 0 \\
0 & 0 & \sigma_{\rho r l}^{2} & 0 & 0 & 0 & 0 & 0 \\
0 & 0 & 0 & \sigma_{\phi r l}^{2} & 0 & 0 & 0 & 0 \\
0 & 0 & 0 & 0 & \sigma_{\rho r l}^{2} & 0 & 0 & 0 \\
0 & 0 & 0 & 0 & 0 & \sigma_{\phi r l}^{2} & 0 & 0 \\
\hline 0 & 0 & 0 & 0 & 0 & 0 & \sigma_{\phi v m}^{2} & 0 \\
0 & 0 & 0 & 0 & 0 & 0 & 0 & \sigma_{\phi v m}^{2}
\end{array}\right]
$$

where, the values of $\sigma_{\rho r l}^{2}, \sigma_{\phi r l}^{2}$ and $\sigma_{\phi v m}^{2}$ are constant and were obtained along with each error term in a large-scale trial.

And, finally, the matching step plays an important role, because the vector from equation (4.41) can be correctly built according to the match. The Mahalanobis distance estimates achieved correct matching. Although it was used for every sensor, the matching for the visual markers can be easily be performed by matching the visual code identified by the camera.

\subsection{Semantic Map}

The world where Doris is going to perform actions needs to be represented. An XML file is a common solution for world description. This world is represented by some important items as features (tables, chairs, doors, chargers, objects...), sites where the robot needs to 
go to, and the ways to arrive to those sites and the landmarks located on each room which help Doris with locatilization.

In listing 4.1 there is sample of one of the locations where Doris is intended to move around, remarking the set of landmarks, features, sites and ways. This map is built with a priori knowledge of the user. The information contained in the XML file is detailed as next:

- landmarks: contains information of the location of a set of landmarks and the type of each one. The type of the landmark can be either laser or camera.

- features: this set contains information of objects placed in the environment like doors, tables, closets, etc. also detailing by each one the location, the width and the height.

- sites: this is a set of waypoints where Doris will be able to move around. Each waypoint contains the position referenced form an origin of coordinates, the semantic name of that site, and optionally some waypoints semantically known as door in case that site leads to another sector. this feature is optional because there can be doors that go outside of a building and this information is no needed.

- ways: this contains the information of how sites connect each other (adjacencies of each site)

- tags: this set contains the information of tags spread over the sector, referencing semantic information contained in the sector.

Listing 4.1: Semantic map sample

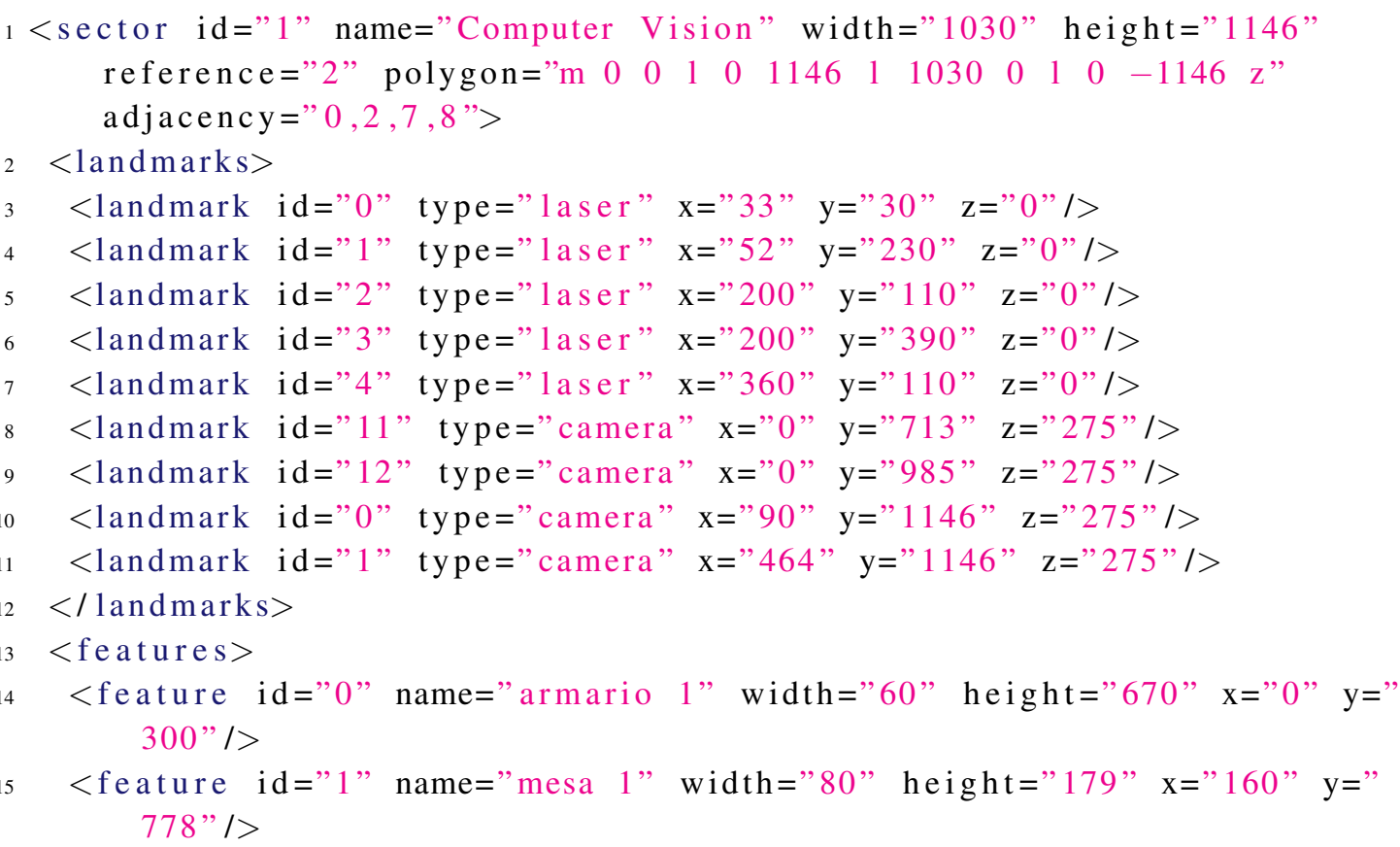




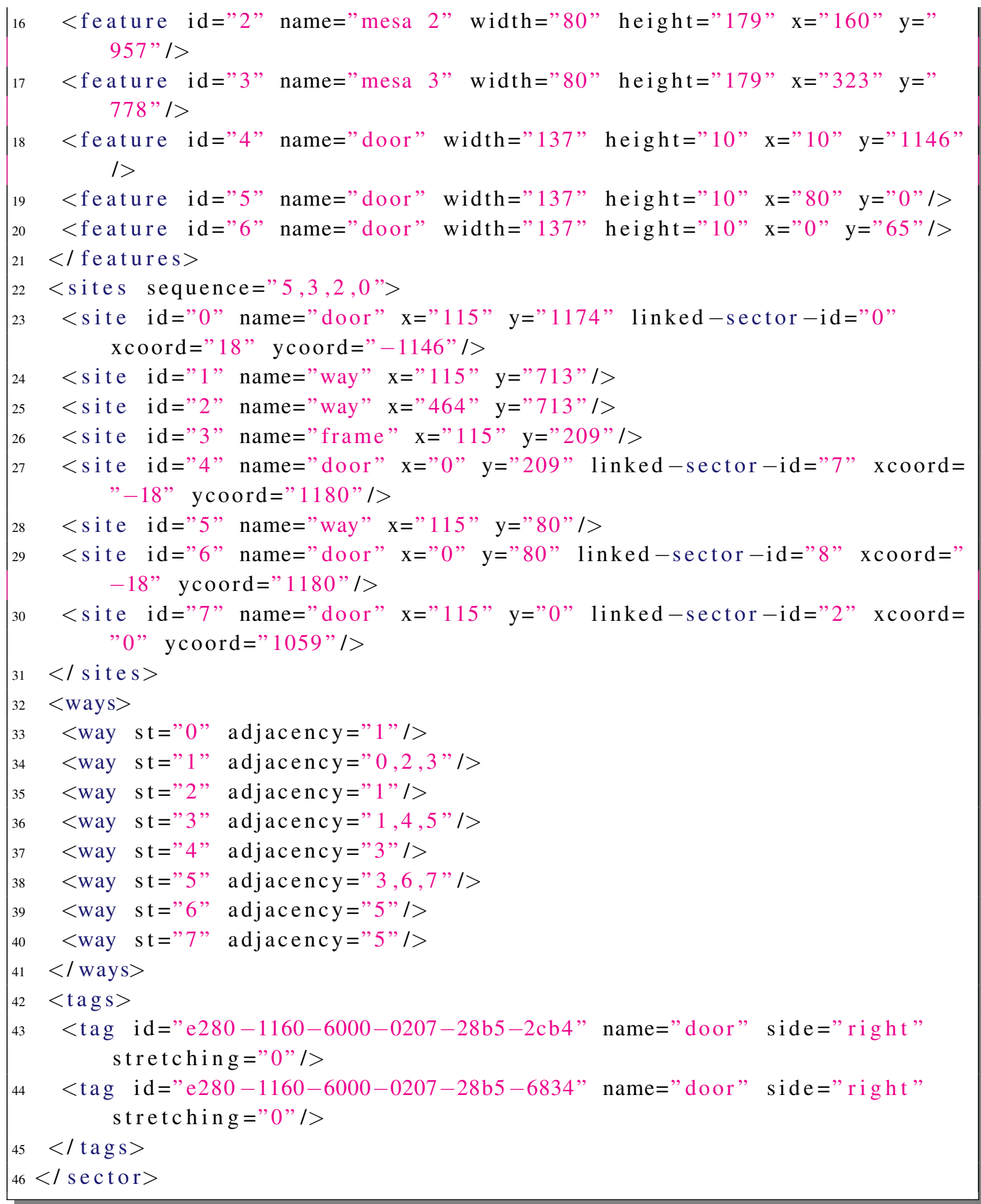

\subsection{Reactive control. Path following}

To complete the whole trajectory, a Fuzzy Speed Controller was designed. The distance and angle errors are the inputs to the fuzzy system providing the linear and angular speed as outputs. The inputs are represented as the membership functions shown in figures 4.9 and 4.10 


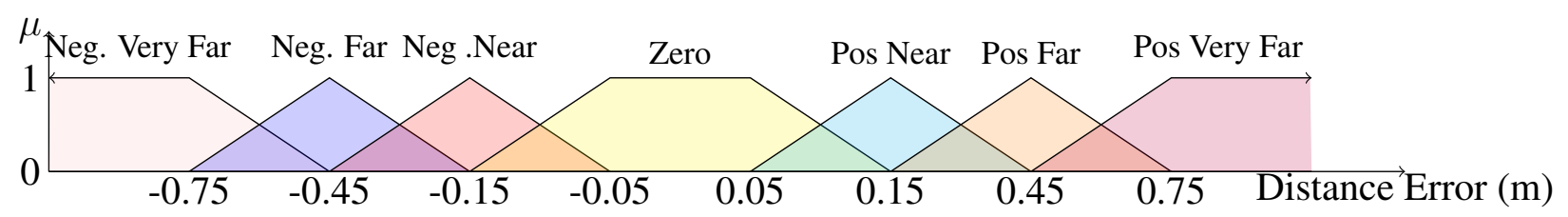

Figure 4.9: Membership functions of the distance input to he speed controller

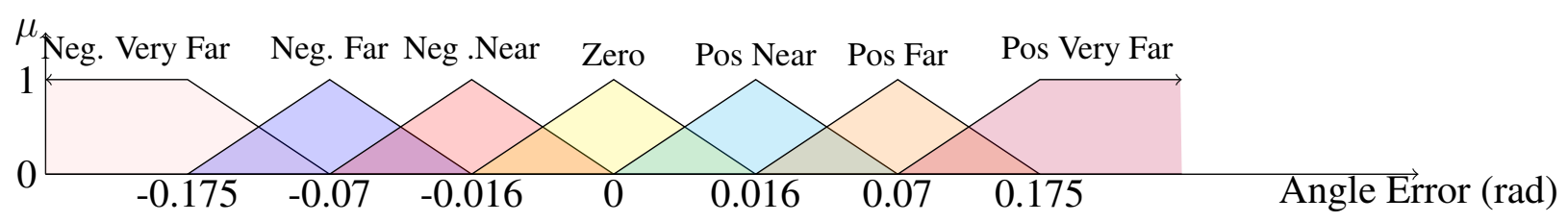

Figure 4.10: Membership functions of the angle input to the speed controller

The outputs of the fuzzy speed controller are depicted in the fuzzy sets shown in figures 4.11 and 4.12

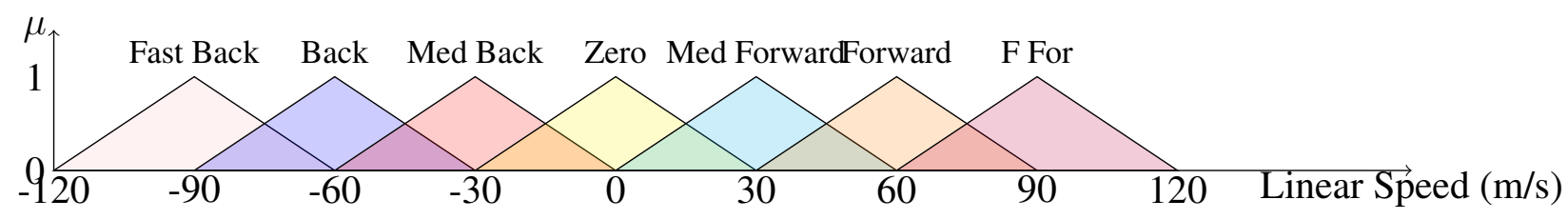

Figure 4.11: Linear speed output memberships function of the speed controller

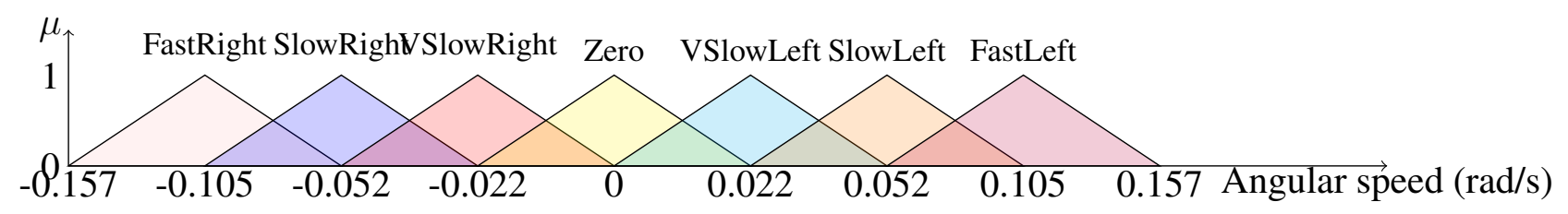

Figure 4.12: Angular speed output membership functions of the speed controller

The label hallway can be found in two ways. One way is on the semantic map by identifying the corresponding sectors or sites using the hallway label. The second way is also on the semantic map but in the Tags subtree, where the corresponding tags of the RFID system are used to identify beginning and ending of a hallway.

As stated, when detecting an incoming hallway, Doris activates a controller specially designed for this type of environments. This controller uses fuzzy logic to override the path controller and keep the robot centred in the hallway. Centering the robot might be affected by dynamic or static objects not present in the semantic map, but this should not be a problem for Doris crossing the hallway from the beginning to the end in a safe and secure mode. 


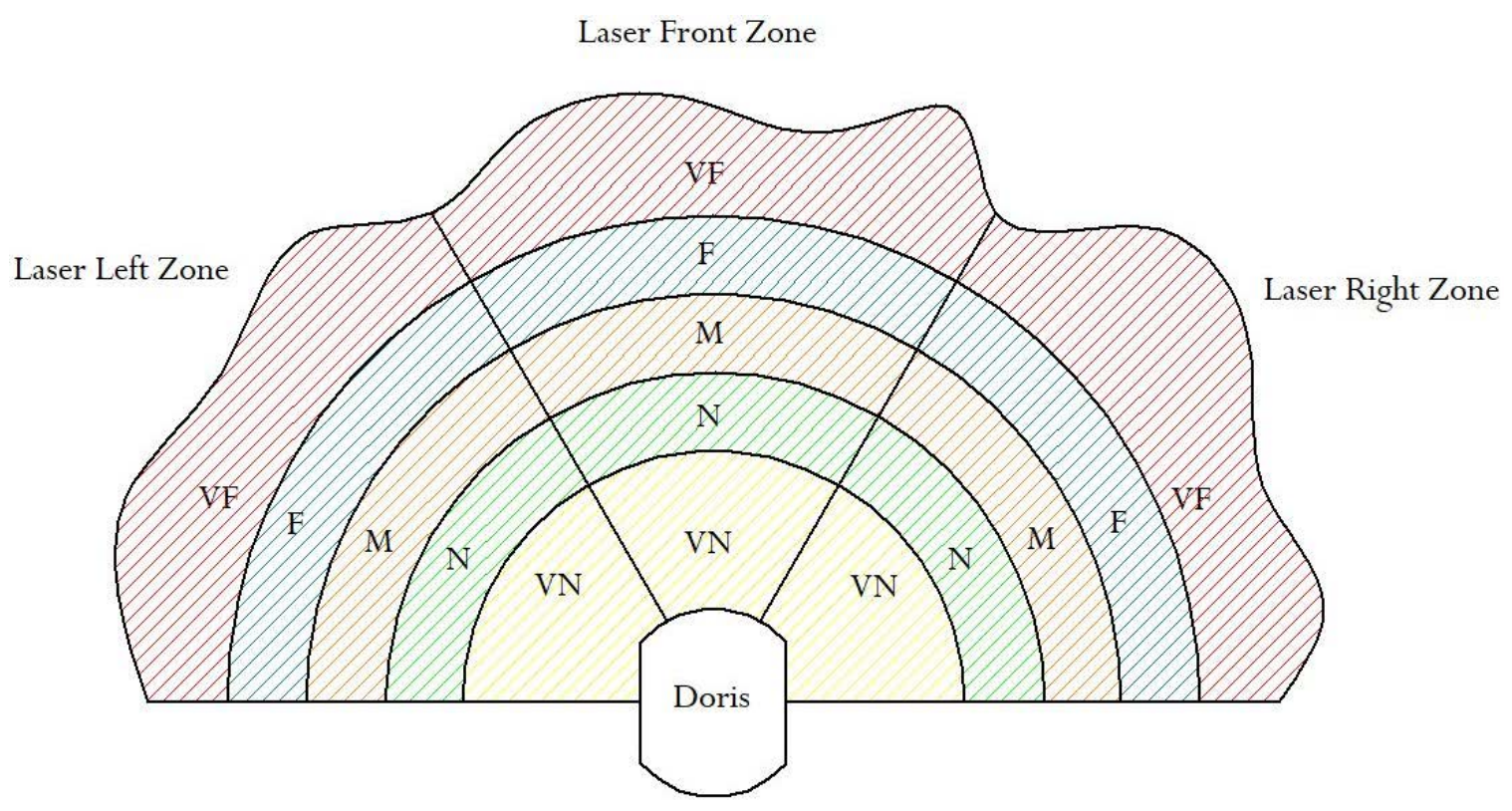

Figure 4.13: Fuzzification of zones performed by the laser

So, the first step in developing the controller is to receive a signal to activate the hallway controller. This signal can be provided either by the semantic map or by the RFID tags. Once inside the hallway, the controller will start to iterate until the signal of end of hallway is received.

The fuzzy system is designed by using the FuzzyLite library designed by [91], the engine is formed by five fuzzy variables, three inputs and 3 outputs. Doris laser range shots a beam every 0.5 degrees in a 180 degrees scan resulting in 361 measurements. These measurements are divided in three zones (left, front and right) each one of 120 measurements. These zones are used as inputs to the fuzzy systems. Angular and linear speeds are used as outputs.

In order to avoid false measurement due to dust or any other interference, each zone is divided into five zones, which will represent every fuzzy membership of a fuzzy set (shown in figure 4.18) in order to reject all those meaningless measurements.

Each input fuzzy set looks like figure 4.14

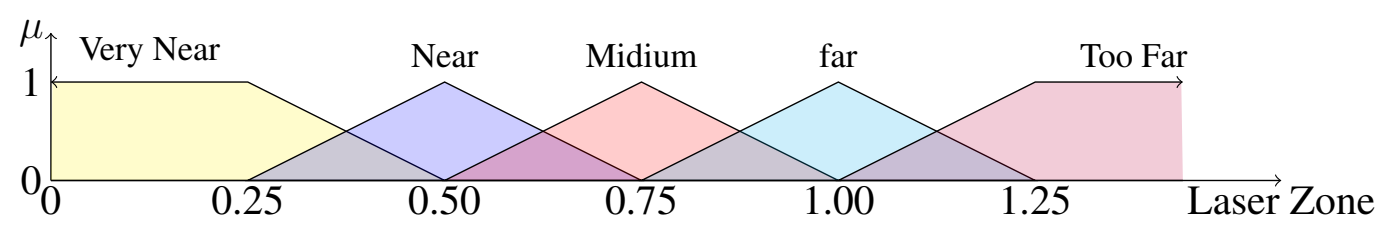

Figure 4.14: Each membership function of the input zones

The fuzzy outputs are defined as in figure 4.15 for linear speed and figure 4.16 for 
angular speed.

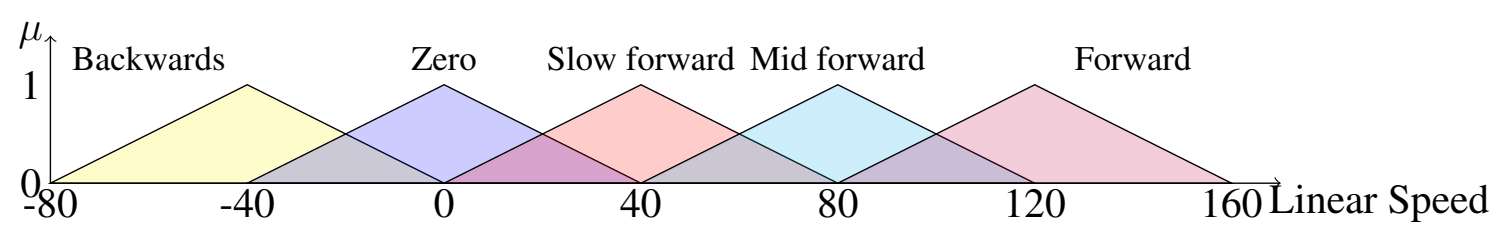

Figure 4.15: Membership functions of linear speed

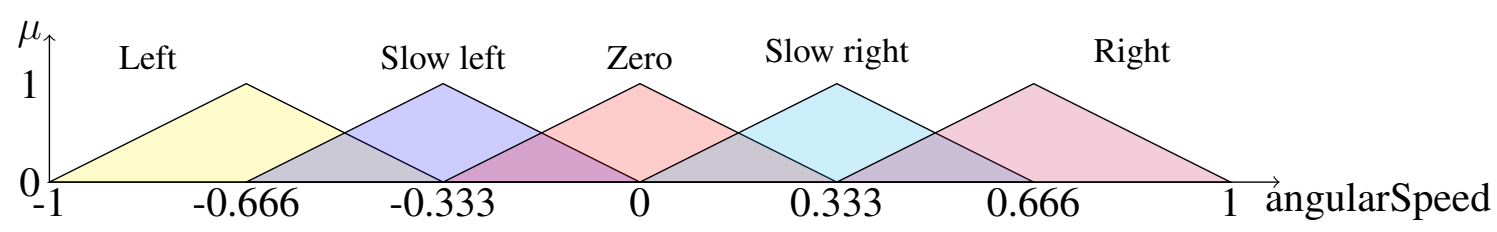

Figure 4.16: Membership functions of angular speed

Special cases apply to this controller, for example, when the controller finds an opened door and the robot must go forward, the controller remains in the center of the hallway, but if the hallway has gone bigger, the controller modifies the path to move Doris to the new center.

\subsection{Planification}

The planning can be considered from two different points of view, the path planning, which consists in which route should the robot must follow to achieve a goal point in a room, and the other type is the task planning, which consists in the order of actions that the robot should make to perform a tour over a museum. Both planners are explained next.

\subsubsection{Path planner}

The path planning is based in the graph built from the semantic map, which contains definitions on how sites inside a sector are connected and also how sectors are connected among them. The semantic map and what information includes will be explained in further details in chapter 4 .

As stated, there are connections of the sites inside and connections between sectors, this implies two graphs must be built, one graph for the sites inside a sector where each node of the graph are the sites, and the other graph for each sector. These graphs are able to find the shortest path by using the $\mathbf{A}^{*}$ and the Branch and Bound algorithms. 
The class diagram in figure 4.17 details de dependency of the graphs inside the path planner and the information provided from the XML databases (semantic map).

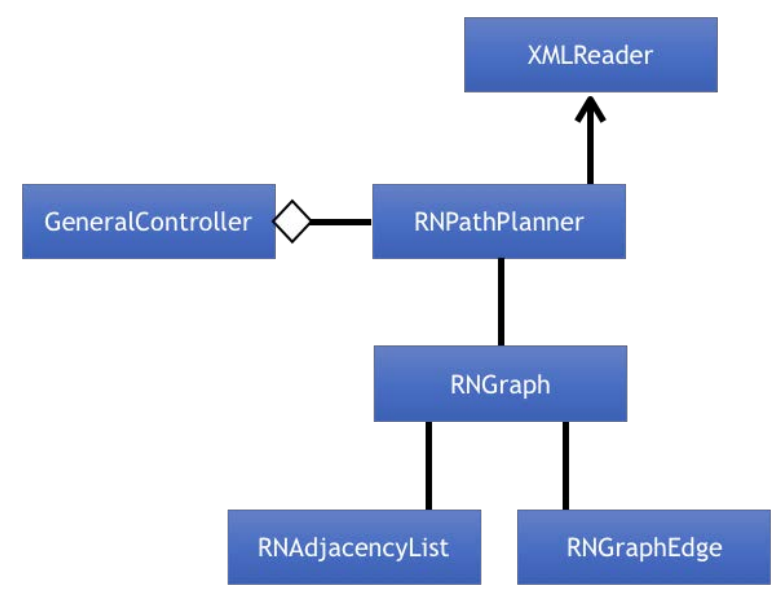

Figure 4.17: Class diagram of path planner

As shown in subsection 4.2, the semantic map is divided by sectors, so two path planners are needed for trajectories, one planner is used inside a specified sector by navigating to specific points inside the museum room, over a specific way detailed in the semantic map, and the second planner is used for inter-sector navigation, for example, when the robot has ended the tour and it is needed to go to the beginning point.

For external navigation a Branch and Bound algorithm is used [92], [93], [94], [95], [96], [97]. A graph representing the external connection between sectors is built and used by the algorithm. The graph is also built by information provided in the semantic map, where each sector has an attribute named adjacency indicating the sectors linked to the current sector.

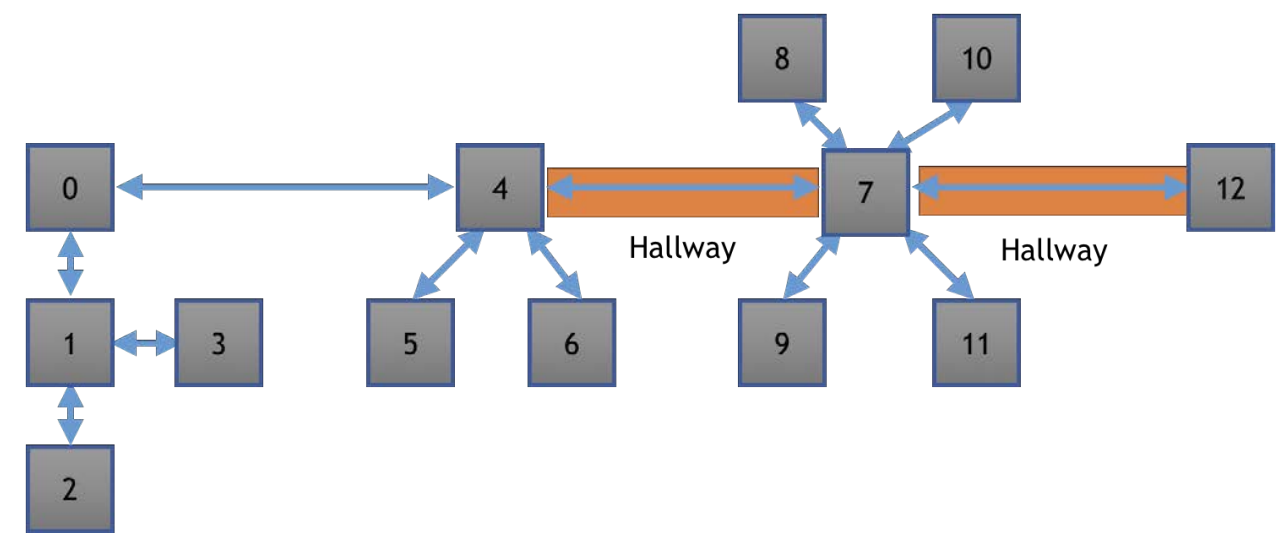

Figure 4.18: Representations each sector connection

In figure 4.18 a representation of how the sectors of a specific map are connected can be seen. Moreover, in this figure the Hallway label represents a special condition that must activate a Hallway Controller can be seen. 
Inside each node depicted in the figure 4.18 there are also nodes that represent the ways and the places where the robot can move and this is where the second path planner takes place which looks for the shortest path between two points of interest using the ways specified in the semantic map. These ways are marked inside the ways subtree inside the sector where the attribute st corresponds to one site and the attribute adjacency indicates the adjacent nodes to that specific site. To find the optimum path, an $\mathbf{A}^{*}$ algorithm is performed [98], [99], [100], [101], [102], [103], [104], where each weight between each node is assigned dynamically depending on the proximity to the site where the robot is intended to move to.

In figure 4.19 a representation of the ways and the crossing points of Doris can be seen. In the figure, a door means that the crossing point is connected to another sector. This helps to the external planner to find the path between two sectors.

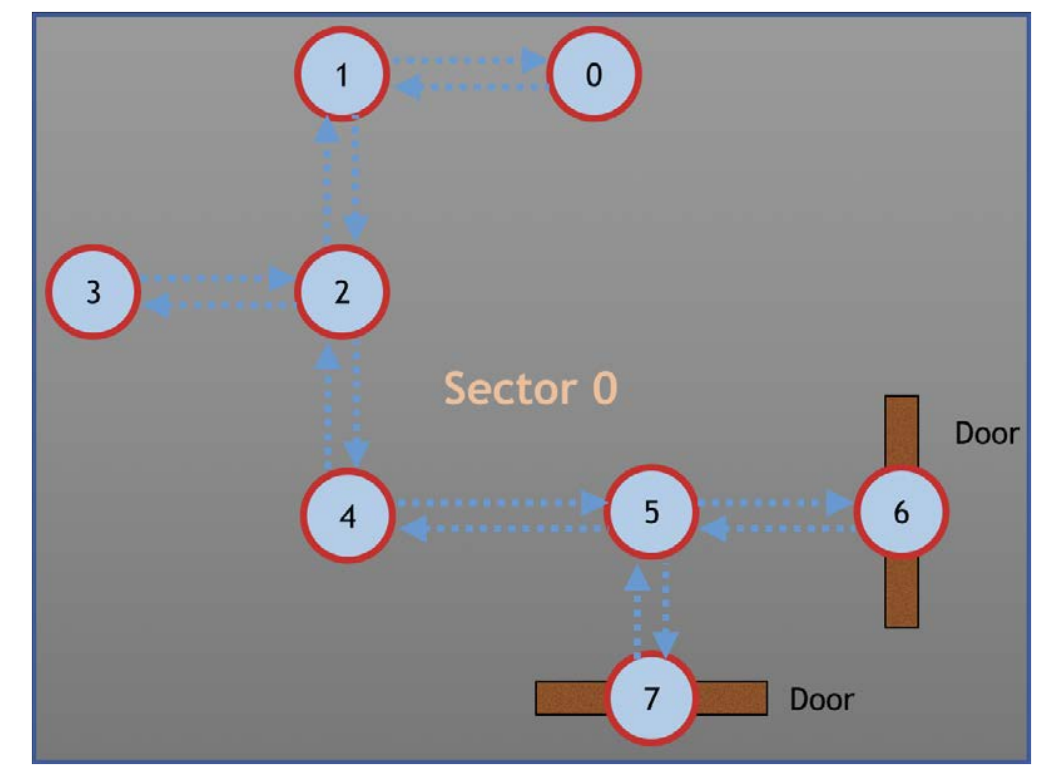

Figure 4.19: Representations of the ways where Doris navigates in a sector

\subsubsection{Task planner}

In chapter 2 was explained about compilers and interpreters used to plan the tasks of robots. In these order of ideas, a task planning module is needed to plan the tasks of Doris and to integrate all the sub-systems previously described. The details of how the interpreter is developed is explained in chapter 7.

The class diagram in figure 4.20 explains how the thread RNTourThread connects with the path planner and also with the GeneralController which allow to communicate with the other processes and to achieve the full control of Doris from the interpreter. 


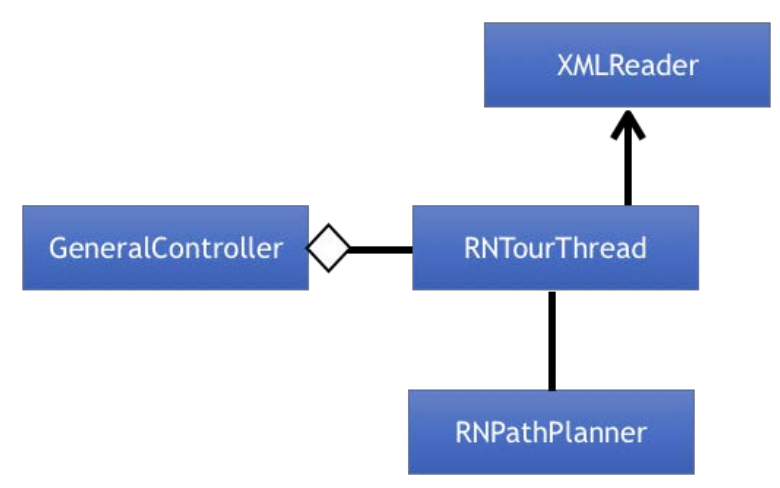

Figure 4.20: Class diagram of task planner

\subsection{Experiments and results}

A set of tests were performed to each module of this chapter. Several test were performed to the localization system due to its importance in the process of allowing Doris to know its exact position in the world. These tests are based on using only the laser sensor, then by using only the omnidirectional camera and finally merging both sensors using the EKF. Then another set of tests were performed in open indoor environments to test the speed fuzzy controller, and another set of tests were performed in a hallway to test the fuzzy hallway controller. Finally, the path planner is tested by giving the instruction to Doris to move from one sector to another.

\subsubsection{Localization results}

Some tests were performed after programming the filter and the detectors in each task. These tests were performed in a sector located at the Higher Technical School of Industrial Engineering in Madrid as depicted in figure 4.21. This operational area has an approximate width and height of $25.66 \mathrm{~m} \times 5.85 \mathrm{~m}$, with a hallway that accesses other rooms that it is about $1.73 \mathrm{~m}$ high.

For the initial position, the robot was placed near one door of the hallway, at the position $[1.4 \mathrm{~m}, 3.9 \mathrm{~m}, 0 \mathrm{rad}]^{T}$, and the covariance matrix for the initial position $\mathbf{P}(0)$ was:

$$
\mathbf{P}(0)=\left[\begin{array}{ccc}
0.000567 & 0 & 0 \\
0 & 0.000567 & 0 \\
0 & 0 & 0.010417
\end{array}\right]
$$

These covariances matrices, $\mathbf{P}(0)$ and $\mathbf{Q}(k+1)$, should adapted to reality, otherwise the EKF will take a higher amount of time to converge. 


\section{Laser tests}

Landmarks were placed in the hallway which was used for the experiment, at a distance of $275 \mathrm{~cm}$ from each other. The reason for this distance was due to the classification landmarks algorithm. If two consecutive landmarks are too close, this algorithm might recognize and classify both as one single landmark. Neither can the landmarks be too far from the LRF, otherwise it will not perceive them, and although the laser can reach up to $32 m$, reflectiveness is lost over distances. Figure 4.21 represents how the reflective landmarks are distributed along the corridor.

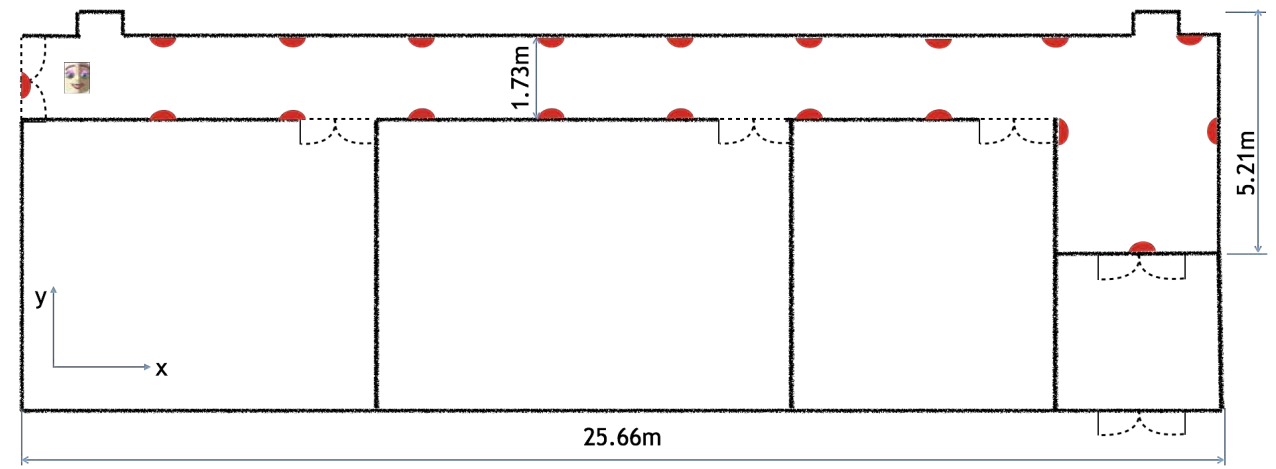

Figure 4.21: Distribution of visual and reflective landmarks

After several tests developed in a narrow hallway with this configuration of landmarks, so that the filter can converge with only the laser readings the following covariance matrix $\mathbf{R}(k+1)$ might represent a good approximation:

$$
\mathbf{R}(k+1)=\left[\begin{array}{ccccc}
0.000083 & 0 & 0 & \cdots & 0 \\
0 & 0.000027 & 0 & \cdots & 0 \\
\vdots & \ddots & \ddots & \ddots & \vdots \\
0 & \ldots & 0 & 0.000083 & 0 \\
0 & \ldots & 0 & 0 & 0.000027
\end{array}\right]
$$

Then, using this variance for each measure, the Mahalanobis distance will reject all those measures which are over $0.02 \mathrm{~m}$ for the distance and $0.01 \mathrm{rad}$ in case of the angle. In case both measures given by laser are above this tolerance threshold, the measures will be rejected and the filter will not be used for correction.

The operation of the filer is shown in figure 4.22. The behavior of the EKF shows how it has converged. One line represents the real odometry provided by the robot, and the other line represents the real pose of the robot provided by the EKF. Figure 4.23 shows the evolution of the position covariance matrix at each step, where each line represents the evolution of the prediction $\mathbf{P}(k+1 \mid k)$ and the correction $\mathbf{P}(k+1 \mid k+1)$. It can be seen that there are some points where the filter increments the uncertainty, which happens because the observations are provided from one to zero landmarks that are not accurate 
enough for the EKF, while the robot keeps moving forward. Despite this inconvenience, the filter manages to converge rapidly when a new landmark is seen.

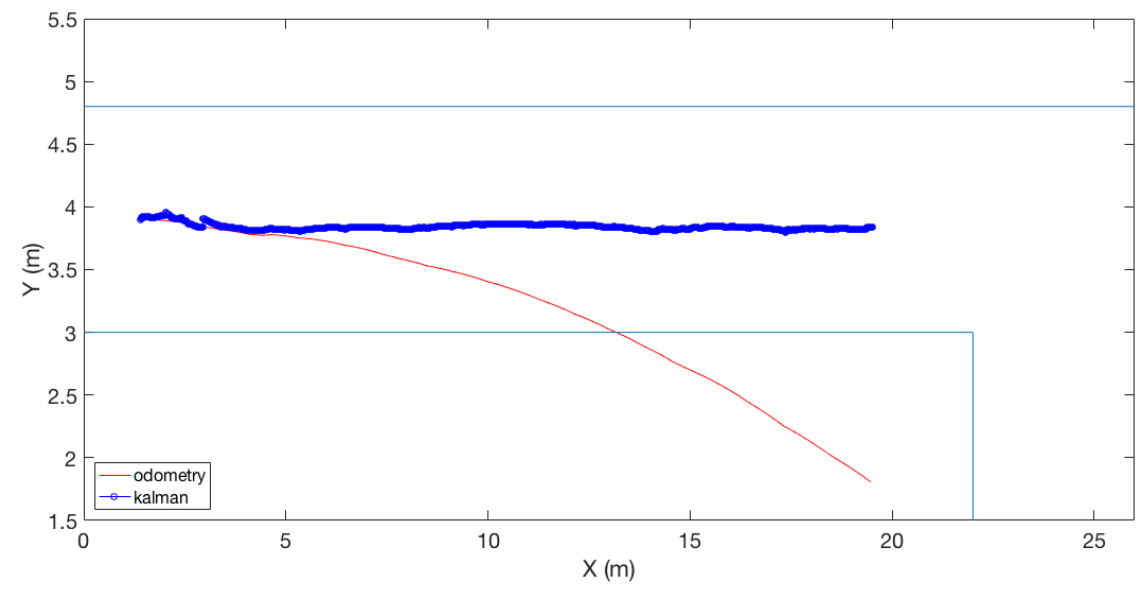

Figure 4.22: Odometrical position of the robot vs. EKF using only LMS-200
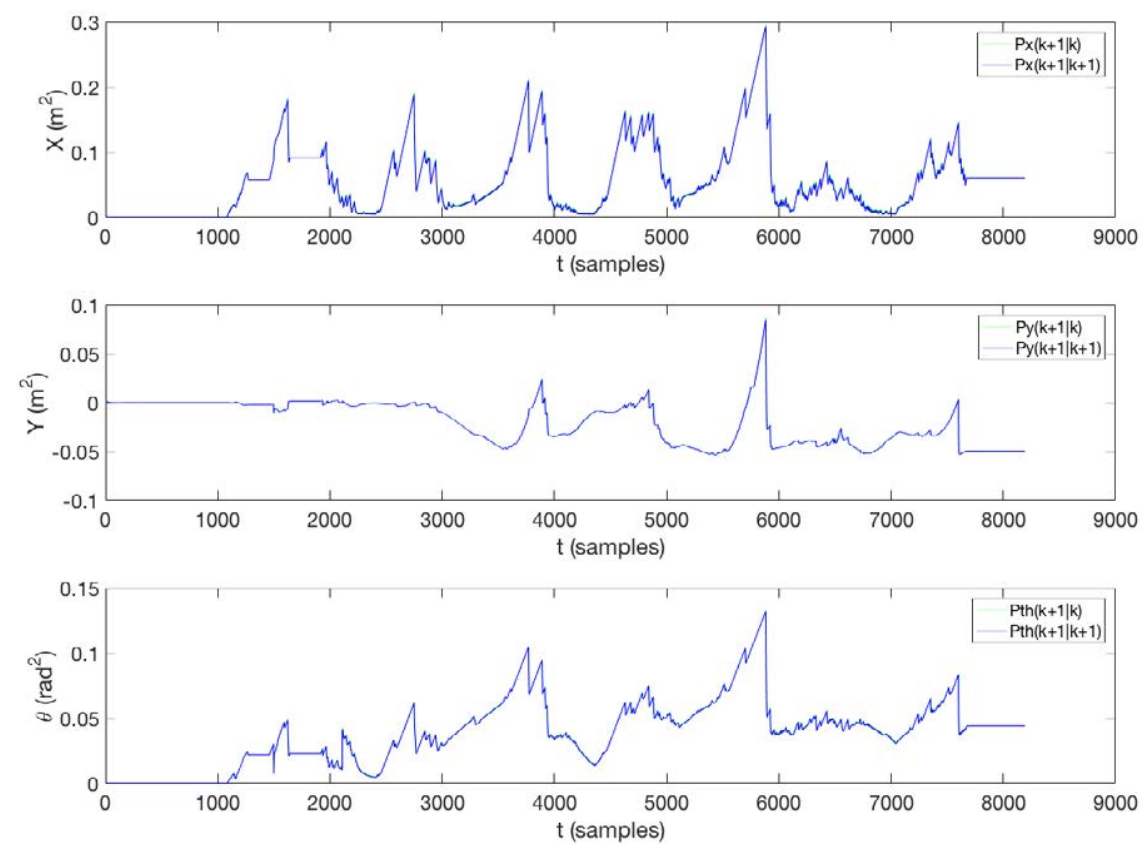

Figure 4.23: Estimated position covariance of the robot using only LMS-200

Supposing that the landmarks might not be seen at any one time, in crowded places where people are walking around the robot, the laser will not be very useful. Hence the need to add a further sensor that can register other landmarks at all times, which explains the need for a camera. 


\section{Camera tests}

Visual markers were placed at the same position above the reflective landmarks, in order to compare the results provided by the EKF working only with laser LMS-200 in section 4.5.1. On this occasion, the EKF was only functioning with the camera. This sensor had to be connected to another computer in order for the image processing to function as quickly as the laser.

Several tests were performed with these markers and the following covariance matrix $\mathbf{R}(k+1)$ represents a good approximation of the filter only converging with the camera:

$$
\mathbf{R}(k+1)=\left[\begin{array}{ccccc}
0.000045 & 0 & 0 & \cdots & 0 \\
0 & 0.000045 & 0 & \cdots & 0 \\
\vdots & \ddots & \ddots & \ddots & \vdots \\
0 & \cdots & 0 & 0.000045 & 0 \\
0 & \cdots & 0 & 0 & 0.000045
\end{array}\right]
$$

Comparing the covariances matrices 4.49 ) of the laser and (4.50) the camera, it can be seen that the variance error of the camera angle was lower than the variance error of the laser angle. This arrangement yielded a better EKF performance that may be seen in figure 4.24

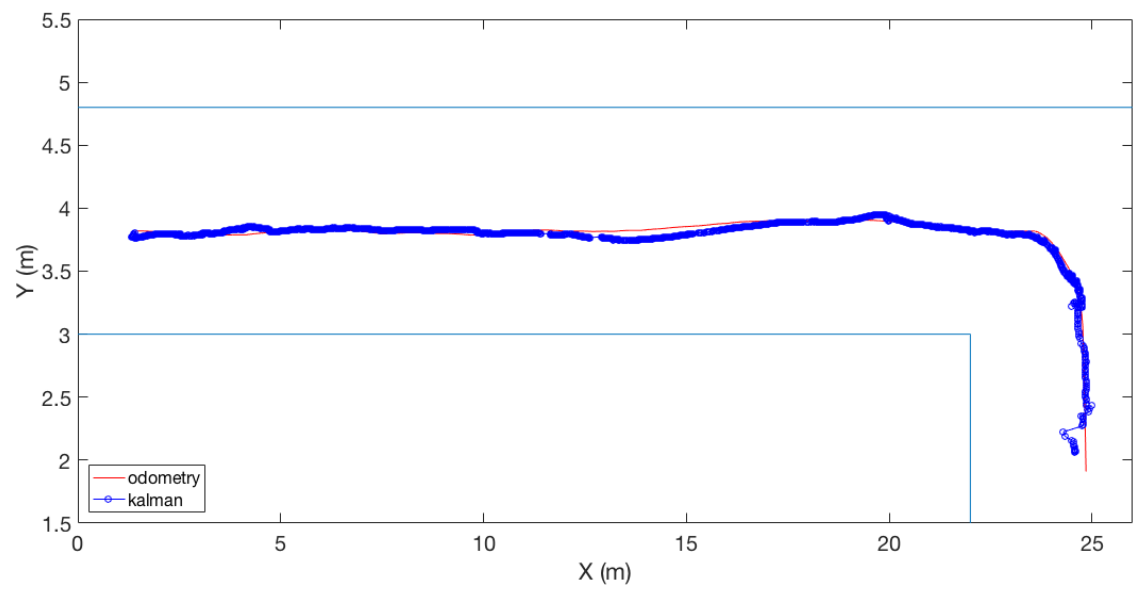

Figure 4.24: Odometrical position of the robot v. EKF using only the Mobotix Camera 

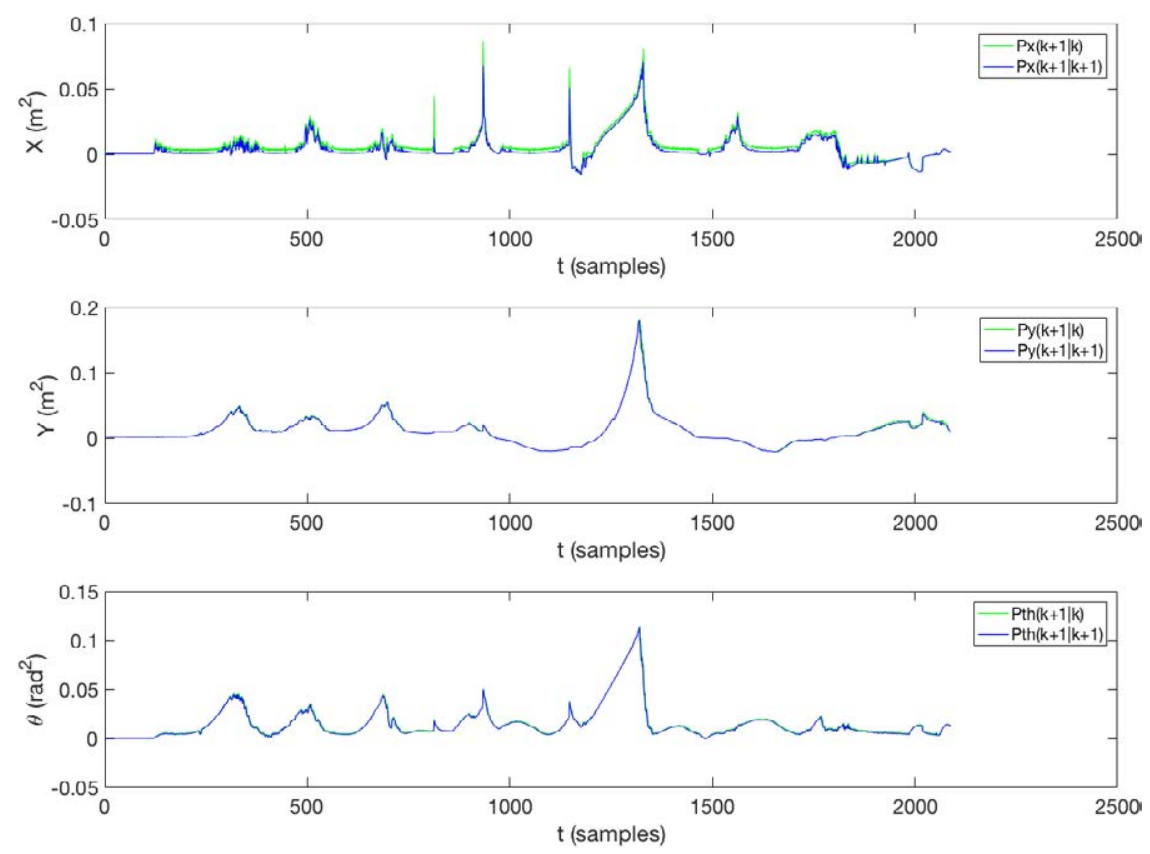

Figure 4.25: Estimated position covariance of the robot using only the Mobotix Camera

Comparing the results obtained in section 4.5.1, it can be seen that the camera, which has minor marker estimation errors and a 360 degree field of view, can register more markers than the laser sensor. While the laser can see at least 2 landmarks, the camera can see 5 or more markers.

When peaks appear in the uncertainty graphs in figure 4.25, it means that no marker is received in the CPU of the robot, due to communication problems (cable, wifi, etc) or a malfunction of the detector where there is too much light, resulting in noise for the adaptive threshold. The most notorious example is the peak between the samples 1000 and 1500, where the robot did not received any visual landmark in a long period.

\section{Laser and Camera}

Figure 4.26 shows how the EKF works much better when both sensors are combined. The uncertainty achieved with both sensors is lower than the uncertainty achieved with only the laser or only the camera. The uncertainty achieved by each sensor is shown in figures 4.23 and 4.25 , where the laser sensor showed a mean uncertainty in positions at around $0.06 \mathrm{~m}^{2}$ and at an orientation of $0.04 \mathrm{rad}^{2}$, and the camera presented a mean uncertainty at a position of $0.004 \mathrm{~m}^{2}$ and at an orientation of $0.01 \mathrm{rad}^{2}$, underlining the superior performance of the camera compared to the laser. A combination of the results of both sensors in figure 4.27 showed a mean uncertainty in positions around $0.02 \mathrm{~m}^{2}$ and at an orientation of $0.02 \mathrm{rad}^{2}$. Comparing the results, better operational performance was achieved with only the camera, although the laser can help at certain times, when 
visual markers are unavailable. For this reason the robot will work with both sensors for positioning.

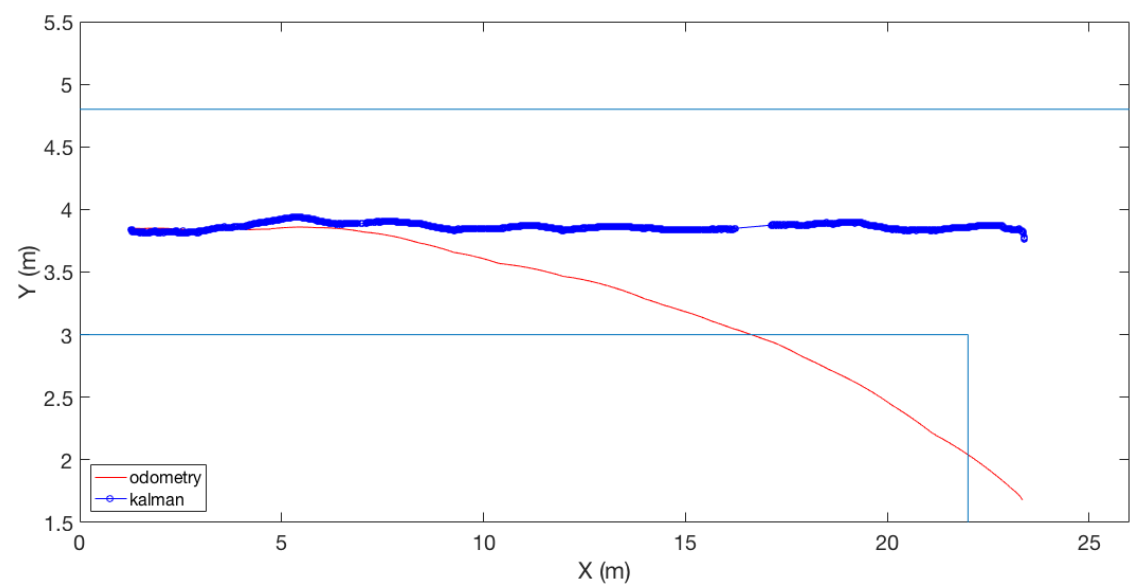

Figure 4.26: Odometrical position of the robot versus EKF using both LMS-200 and the Mobotix Camera
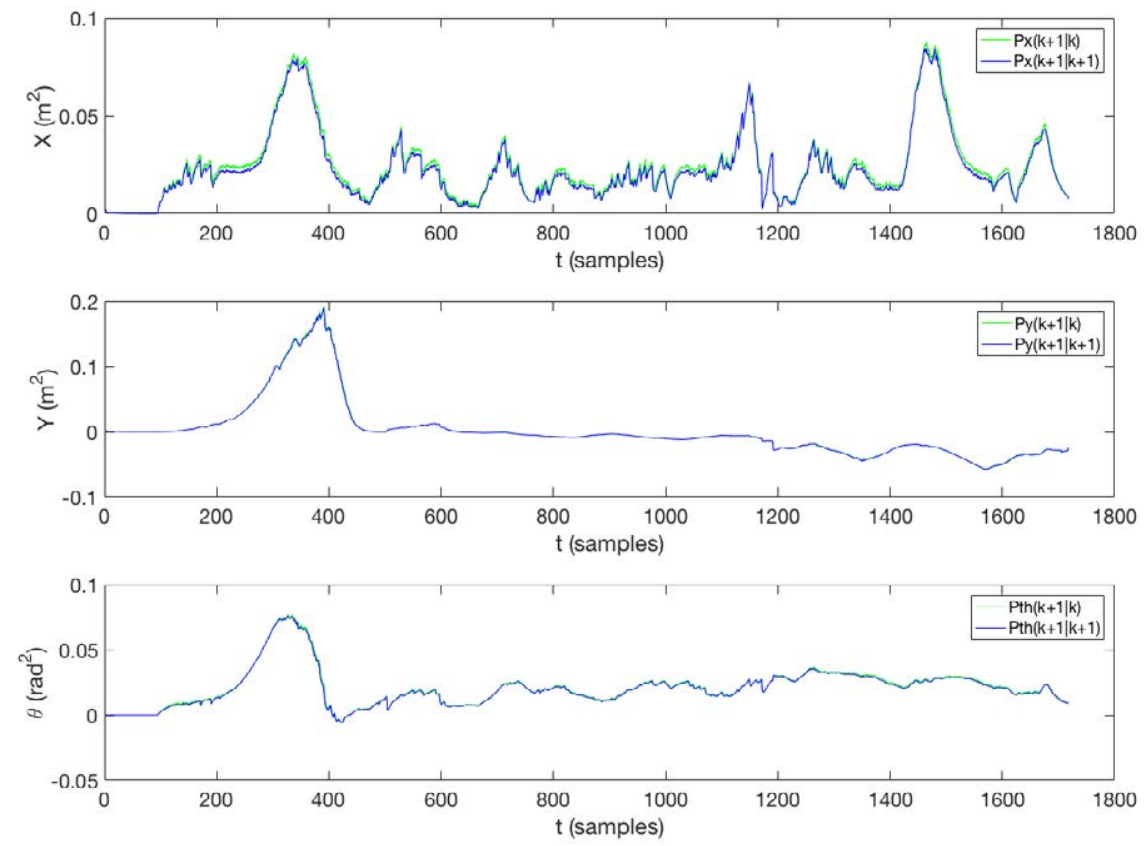

Figure 4.27: Estimated position covariance using both LMS-200 and the Mobotix Camera

\subsubsection{Reactive control results}

Besides localization where the sensor fusion system has been proved to work at [78] and [77] also needs to be tested the planification, and the fuzzy controllers which are the position controller and hallway controller, both based on the linear and angular speed provided to the robot. 
Concerning the hallway controller, test were performed in the main hallway of a building within the Central Laboratory of Electrical Engineering which is $175 \mathrm{~cm}$ width and $24 \mathrm{~m}$ of length. the performance can be seen in figure 4.28 and a video, named Hallway Controller Test, can be found at the group's webpage gallery Intelligent Control Group UPM-CSIC - Gallery

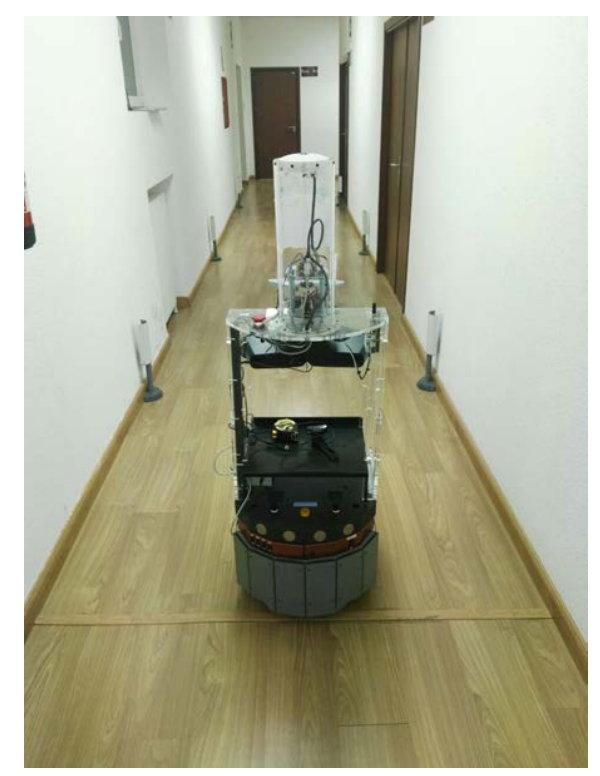

Figure 4.28: Hallway where controller is being tested

\subsubsection{Path planning results}

A experiment with obstacles and people passing by was developed in the Center of Automation and Robotics located at Universidad Politécnica de Madrid. The trajectory is shown in figure 4.29. The experiment shows the use of path planner proposed on this dissertation. The use of both planners, the internal sector planner based on a $\mathbf{A}^{*}$ algorithm and the sectors planner based on a branch and bound algorithm can be appreciated in this experiment. Thus, it can also be seen the use of the localization system and controllers designed.

The robot goes from sector one (1), to sector two (2) according to the figure shown in 4.18 , moving from the charging station located at $[2 \mathrm{~m} 7.11 \mathrm{~m}-1.57 \mathrm{rad}]^{T}$, it moves backwards, then spins 90 degrees and keeps moving backwards to the point $[3.65 \mathrm{~m} 8.01 \mathrm{~m}-3.14 \mathrm{rad}]^{T}$. The fuzzy speed controller is used to follow the trajectory while people is passing around the robot.

This trajectory consists on a set of three points that the robot must pass through. The controller moves the robot from the current position to the point $[1.25 \mathrm{~m} \mathrm{8.01m-3.14rad}]^{T}$ and then the controller tries to keep the robot in a straight line until it reaches the second point $[1.25 \mathrm{~m} 1.41 \mathrm{~m}-1.57 \mathrm{rad}]^{T}$ and then the planner 
commands to the robot to move to another sector, which information is loaded while trespassing the door located at the beginning of the hallway, where a RFID tag is placed, indicating to the robot that a new sector is approaching. Finally, when the sector is already loaded the controller moves the robot up to the final point $[1.0 m 2.3 m-1.57 \mathrm{rad}]^{T}$.

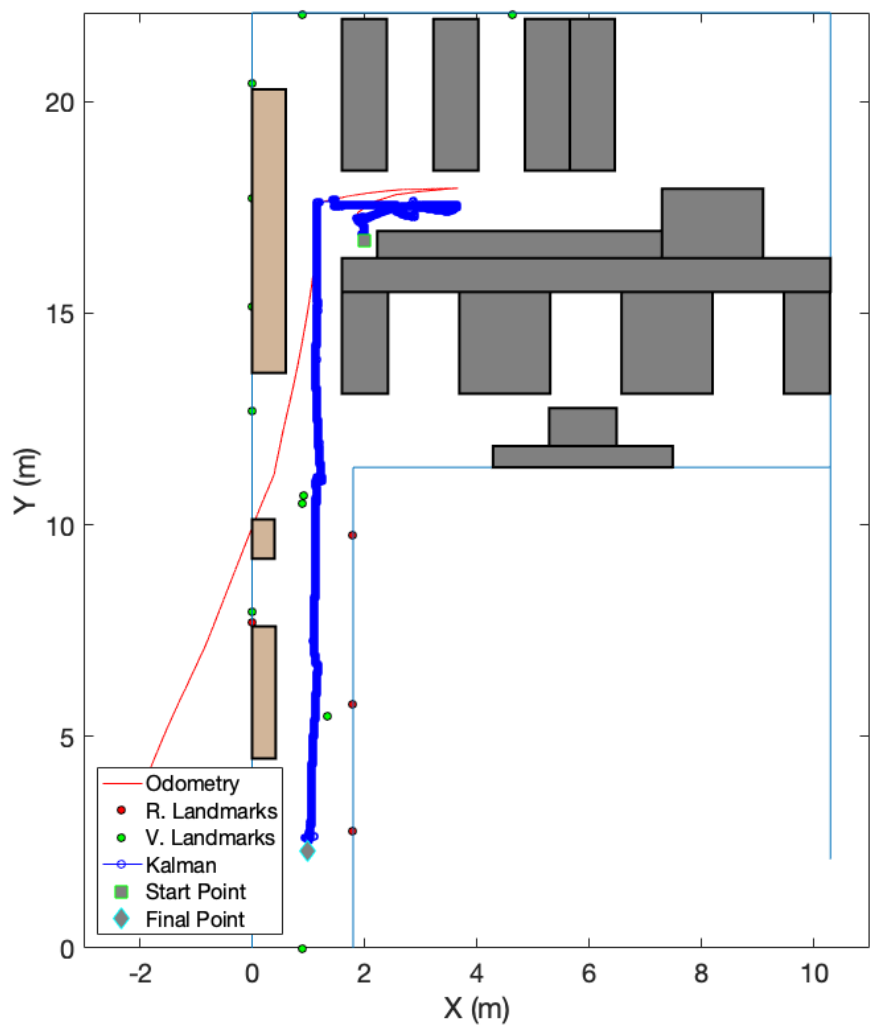

Figure 4.29: Odometrical vs EKF trajectory of the robot using both sensors

In the same figure, the boxes represent objects placed along the environment where the tests are being performed.

This experiment has important issues to highlight. The first one is the landmarks visibility. In the largest area there is no space enough to put some reflective landmarks on the ground, so the first part of the experiment only uses seven visual markers placed on the walls. Then in the hallway located next, there are fewer objects placed on the ground, therefore, five reflective landmarks and five visual markers can be placed. The second issue is that there are static objects and people walking in front of the robot.

The evolution of the covariance matrix of the position is shown in figure 4.30 and the benefits of having both types of landmarks instead of having only one can be seen. The first 15000 samples shown in the $X\left(\mathrm{~m}^{2}\right)$ subgraph the variance of the position with the help of the visual markers. A good localization is achieved but the variance of the estimation is increased. On the other hand, the next samples show the variance when the reflective landmarks are detected improving a lot the localization of the robot. 
While executing the trajectory, one of the reflective landmarks was suddenly occluded, so the localization was only based on the visual landmarks, as can be seen in the same figure from the sample 25000, where the variances of the state is increasing until the last point is reached.
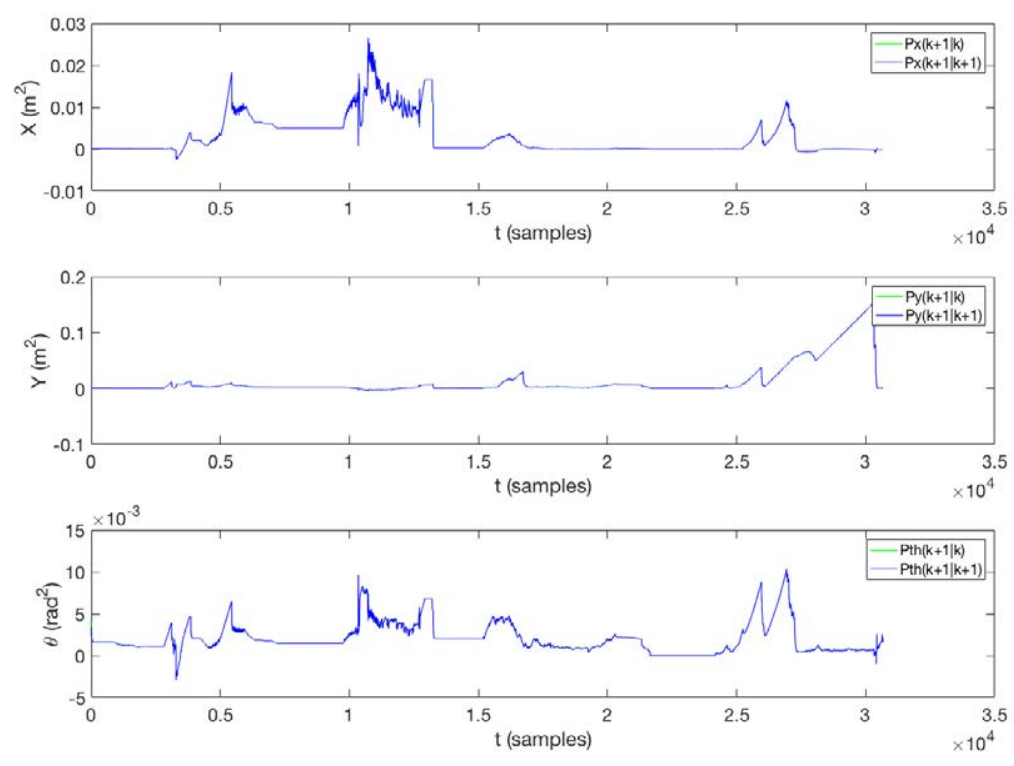

Figure 4.30: Odometrical covariance position using both sensors

Next table shows a comparison between each point given by the trajectory and the position obtained by the EKF:

Table 4.11: Ground truth Robot position vs. Real Position

\begin{tabular}{cccccc}
\hline $\begin{array}{c}X_{\text {real }} \\
(\mathrm{m})\end{array}$ & $\begin{array}{c}Y_{\text {real }} \\
(\mathrm{m})\end{array}$ & $\begin{array}{c}\theta_{\text {real }} \\
(\mathrm{rad})\end{array}$ & $\begin{array}{c}X_{\text {est }} \\
(\mathrm{m})\end{array}$ & $\begin{array}{c}Y_{\text {est }} \\
(\mathrm{m})\end{array}$ & $\begin{array}{c}\theta_{\text {est }} \\
(\mathrm{rad})\end{array}$ \\
\hline 2 & 7.11 & -1.57 & 1.9469 & 7.0982 & -1.5571 \\
3.65 & 8.01 & -3.14 & 3.6231 & 7.9074 & -3.0939 \\
1.25 & 8.01 & -3.14 & 1.3018 & 7.9074 & -3.1081 \\
1.25 & 1.41 & -1.57 & 1.2396 & 1.4482 & -1.4213 \\
1.05 & 10.49 & -1.57 & 1.1407 & 10.5053 & -1.5887 \\
1.00 & 2.30 & -1.57 & 1.0140 & 2.5391 & -1.5149 \\
\hline
\end{tabular}


Table 4.12: Ground truth error

\begin{tabular}{ccc}
\hline Error X (m) & Error Y (m) & Error $\theta(\mathbf{r a d})$ \\
\hline 0.0531 & 0.0118 & 0.0129 \\
0.0269 & 0.1026 & -0.0461 \\
-0.0518 & 0.0508 & -0.0319 \\
0.0104 & -0.0382 & -0.1487 \\
-0.0907 & -0.0153 & 0.0189 \\
-0.0140 & -0.2391 & -0.0551 \\
\hline
\end{tabular}

As it can be observed in the table 4.11 the first three columns are the points where the robot must pass through, the next three columns are the position provided by the Kalman Filter and in table 4.12 the columns are the errors between real and estimated position. Marks were located on the ground to ensure the robot pass over these points. In the fourth row, a significant deviation of the robot can be observed. This is due to people passing in front of the robot and it started to deviate, trying to arrive at the desired position from other point. The same behaviour is presented when robot is in the hallway between ycoordinates 6.00 and 8.00 shown in figure 4.29. It deviates a little but it instantly returns to the trajectory.

\subsection{Conclusions}

Indoor position estimation is extremely useful for guiding a robot in museums and trade fairs. In this study, the robot has shown that its position can be established, especially when interacting with people, by using laser, camera, and RFID sensors. The first step when working with a laser range finder was to build the reflective landmarks; a complicated process requiring expensive materials. The process of finding the best place to locate the reflective landmark was important, so that the classification algorithm could detect different landmarks, despite it being a good method to achieve localization without mapping the whole environment.

An omnidirectional camera is a very powerful sensor because it provides a 360 degree field of view of the built environment and it achieves reliable angle estimations. The visual landmarks, however, had to be printed on an A3 size paper, so that it could be easily detected by the camera at long distances $(5-6 m)$. An A4 size marker was used at first, achieving only a detection range of $0-3 \mathrm{~m}$. One big disadvantage of working with an omnidirectional camera is that a high resolution image is needed for detection that requires a fast processing speed. At present, Doris is not equipped with sufficient processing capacity and the image processing has to be performed by another computer.

The RFID system cannot be used for localization due to the high uncertainty that it 
presents. This is essentially caused by the low operating frequency of RFID. Ultra Wide Band systems work at very high frequencies that increase the efficiency of the performance. However, RFID was used for the navigation system by providing semantics to the environment which is stated into the semantic map.

Regarding the cost of each sensor, which may vary among different suppliers, the following table portraits an approximate balance of the prices of each one:

Table 4.13: Sensor list prices

\begin{tabular}{cccc}
\hline Quantity & Description & Price Unit $(€)$ & Subtotal $(€)$ \\
\hline 1 & LRF SICK LMS-200 & 2276 & 2276 \\
1 & Omnidirectional Camera & 428 & 428 \\
1 & RFID System & 1773.81 & 1773.81 \\
& & Total: & 4477.81 \\
\hline
\end{tabular}

A procedure to obtain the standard deviation for each sensor has been presented. Once sensor fusion was integrated with the EKF some adjustments were successfully applied for faster convergence of the EKF.

Experiments demonstrate that even with obstacles or people passing by, or even in the absence of one type of the landmarks, a good localization, control, navigation and planning is achieved. It can be achieved by using only the omnidirectional camera, but can be refined by including the reflective landmarks.

The results are that Doris can locate on the presence of people and different objects located when both sensors are working at the same time. Although Doris can locate itself by only using the camera, there will be situations where both types of landmarks will not be easy to place or the museum does not allow to place the beacons or maybe it is too dark to place the visual markers, for this reason a sensor fusion was implemented. This methodology was developed in order to avoid using SLAM technique.

The integration considers the use of the navigation previously developed using the EKF with sensor fusion, but when interacting with people navigation needs to be adjusted in order to have a better performance, so the fuzzy sets of the speed and hallway controllers were modified to avoid conflicts when the robot was surrounded by people, and the integration of the RFID system helps with the sector identification using the semantic map. Thus, localization and navigation are optimized. The sector identification tags were placed in doors at $1.0 \mathrm{~m}$ of height and with the RFID system the power and the sensitivity of the antennas were calibrated in order to detect the tags only when the robot is passing through the door. 


\section{Interaction system}

In chapter 3, the architecture proposes that Doris must contain an interaction system in order to interact with the environment. Doris' system is based on several elements, which are the face, the arm, the emotion system, the speaking system and the lip sync system. In the research performed by [105] talks about the effective communication between people and interactive robots when having a common background of understanding. The simpler the communication is, the better the robot will respond to the user, always having into account to avoid the uncanny valley, which is explained in chapter 2 .

Doris' face has the most common characteristics of a person's face, that is to say, eyes, eyelids, eyebrows, nose, lips, It is made of methacrylate and it has 20 degrees of freedom. Thus, the design process of the face, a first approach to it and its current version, are detailed in this chapter.

The speaking system is also a proposal of this dissertation, which will help Doris to explain things to people inside the museum and answer their requests. This system also works along with the lip syncing system, which helps to produce a more realistic communication, which is related to Doris' acceptance among the tourists visiting the museum.

Another proposal of this dissertation is the emotional system which is designed with fuzzy logic. This emotional system works with input requests from the people of the museum. The fuzzy system will be used to obtain the face expression and the voice rate of Doris.

The functionality of the robotic arm, which is used for interacting with people, is also proposed in this dissertation, together with an explanation of the manual control.

Each of the subsystems related to Doris' interaction are detailed, as well as their design and their functionality. The task planning concerning the face is coordinated thanks to the language developed for Doris, which is detailed in chapter 7 . 


\subsection{The expression system}

The importance of having emotions in a robot was explained in section 5.4. This emotions can determine the voice frequency and the face gestures of Doris. A related work is performed in [106] which examines the user gestures to produce other robot gestures. As Doris will interact with humans, its gestures also need to be human, that is the main reason why Doris has a face which can be very similar to a human, being a key feature so that the behaviour of the user can be close to acceptance instead of rejection as explained in [107] providing a natural communication between machine and human [108].

Working in these lines, the face has been constructed by using next materials:

- The faceplate is plastic made.

- Supports for eyes, eyebrows, eyelids, eyelashes and mouth are methacrylate and aluminium.

- Eyes, eyelids, eyelashes and lips were printed.

The design allows the face to have several degrees of freedom. In table 5.1, it is shown where the degrees of freedom are located.

Table 5.1: Doris head degrees of freedom distribution

\begin{tabular}{cccc}
\hline Quantity & Head part description & Degrees of freedom & Total \\
\hline 2 & Eye & 2 & 4 \\
2 & Eyelash & 2 & 4 \\
2 & Eyelid & 1 & 2 \\
1 & Left commissure & 2 & 2 \\
1 & Right commissure & 2 & 2 \\
1 & Upper lip & 1 & 1 \\
1 & Lower lip & 1 & 1 \\
1 & Neck & 3 & 3 \\
\hline & & Total: & 19
\end{tabular}

A first approach of the face is shown in figure 5.1a. It can be seen that the structure of the face is made of methacrylate and the parts of the face in its respective positions. Then over the time, it was decided that Doris can flush according to the situation provoked by the emotion system. Doris cheeks were modifed and added an array of leds which are controlled with the same maestro controller, granting to the face 1 extra DoF. This face can be observed in figure $5.1 \mathrm{~b}$. 


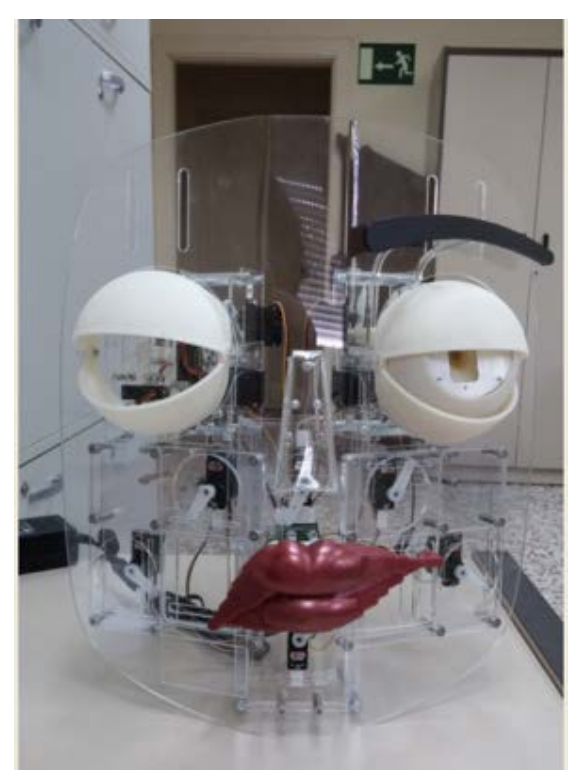

(a) First face approach

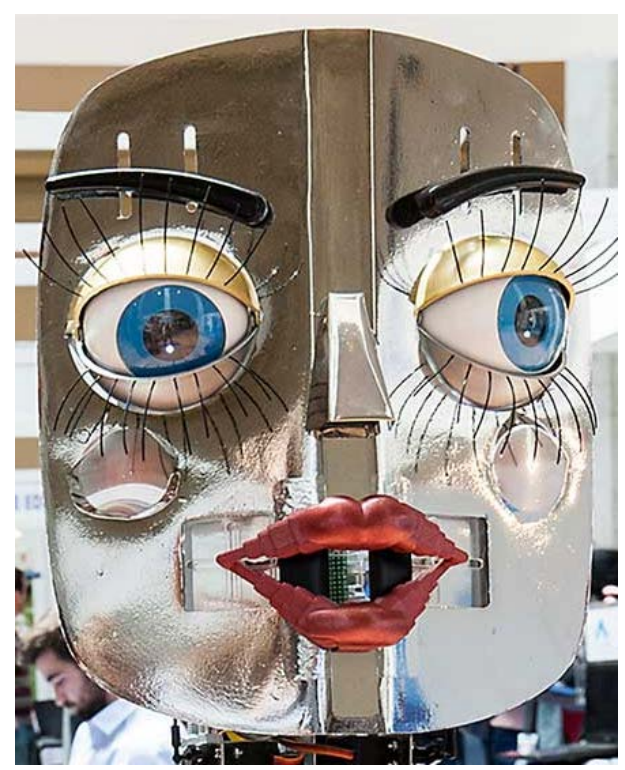

(b) Current mounted face

Figure 5.1: Doris faces

With this consideration, a thread dedicated to control the face was developed. In this thread (RNGestureTask), there are three ways to control the current Doris expression.

- Emotion: the resulting emotion provided by the emotion system (RNEmotionTask) is sent to the gesture thread positioning the face according to the estimated values for each part of the face (eyes, eyebrows, eyelids and mouth)

- Task planner: the script developed by a programmer can contain instructions where the face expression is modified. This is achieved through the say instruction, where it can be configured where the sight is pointing to and what expression is set from a collection of predefined faces.

- Manual: as Doris contains a collection of predefined faces, this can be manipulated from the webpage by selecting one of the faces of this collection.

The set of predefined faces are happy, surprise, desperation, disgust, dissimulation, tired, sleepy, angry, sweet dreams, screaming, hypnotic, evil, scary, suspicious, normal, ashamed, thinker, drunk, preoccupied, empathy, half smile, big smile, evil smile, quiet and sad.

In case that a user or programmer wants to select a predefined face, a simple search in the XML database is performed as shown in algorithm 1 


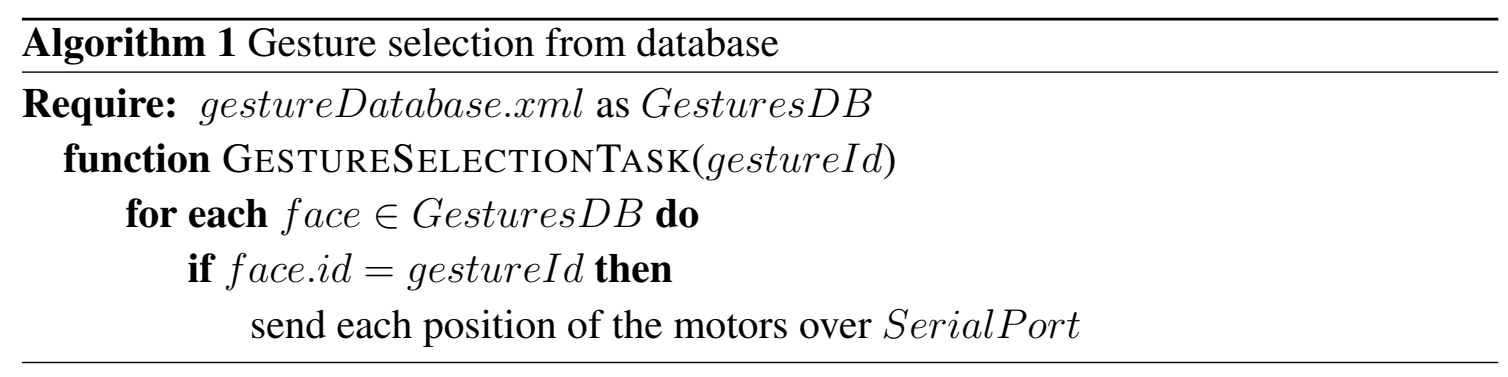

\subsection{Lip syncing interaction}

The lip synchronization consists of a parallel coordination between Doris' mouth movements and the synthesized audio of a text predetermined by the user so that the robot can communicate with its human environment in an almost natural way. As stated before, Doris lips are 6 degrees of freedom, commanded by each respective servomotors, which are controlled in order to perform synchronized movements with the sound emitted by speakers, resulting in a higher speaking reality. The 6 degrees of freedom for Doris lips are like the muscles of a human being. Table 5.2 makes a correspondence of each degree of freedom with those muscles.

Table 5.2: Humans muscles implied on speaking

\begin{tabular}{|c|c|c|}
\hline $\mathrm{N}$ & Muscle & Description \\
\hline 1 & $\begin{array}{l}\text { Upper lip } \\
\text { elevator }\end{array}$ & Rises the upper lip \\
\hline 2 & Obicular & $\begin{array}{l}\text { Open and close lips and helps with the forward and backward } \\
\text { movement }\end{array}$ \\
\hline 3 & $\begin{array}{l}\text { Lowe lip } \\
\text { depressor }\end{array}$ & Makes lower lip go down \\
\hline 4 & Mentalis & Rises the chin skin and affects the rising of the lower lip \\
\hline 5 & Risorius & Retracts the labial commissure producing a smile \\
\hline 6 & $\begin{array}{l}\text { Zygomaticus } \\
\text { major }\end{array}$ & Rises the labial commissure abductor \\
\hline 7 & $\begin{array}{l}\text { Zygomaticus } \\
\text { minor }\end{array}$ & Acts as an elevator and abductor of the upper lip middle part \\
\hline
\end{tabular}

Figures $5.2 \mathrm{a}$ and $5.2 \mathrm{~b}$ show a comparison between human lips and Doris lips and its respective correspondence with table 5.2. Some details like: teeth, tongue, jaw, palate were omitted in the design.

Figure 5.3 shows a flow chart of the procedure to achieve the lip synchronization with the speaking. The first step is to receive the response text from the Dialog Task, this text is normalized by removing interrogation, exclamation and any other symbols which 


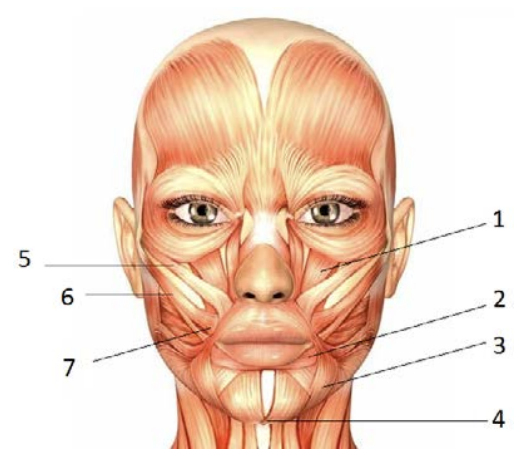

(a) Human muscles implied in speaking

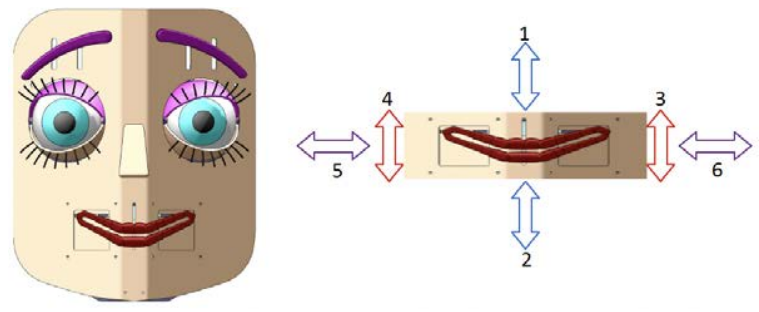

(b) Doris Lips

Figure 5.2: Human-Doris mouth relation

are different to letters. After the text is normalized, it is splitted into words without considering that there might be two consecutive words pronounced as one. Then each word then is separated into syllables based on Spanish syllabication rules and this syllables are compared to the viseme database resulting in a viseme position. The transition time from the current viseme to the next is estimated and used to synchronize the speaking rate.

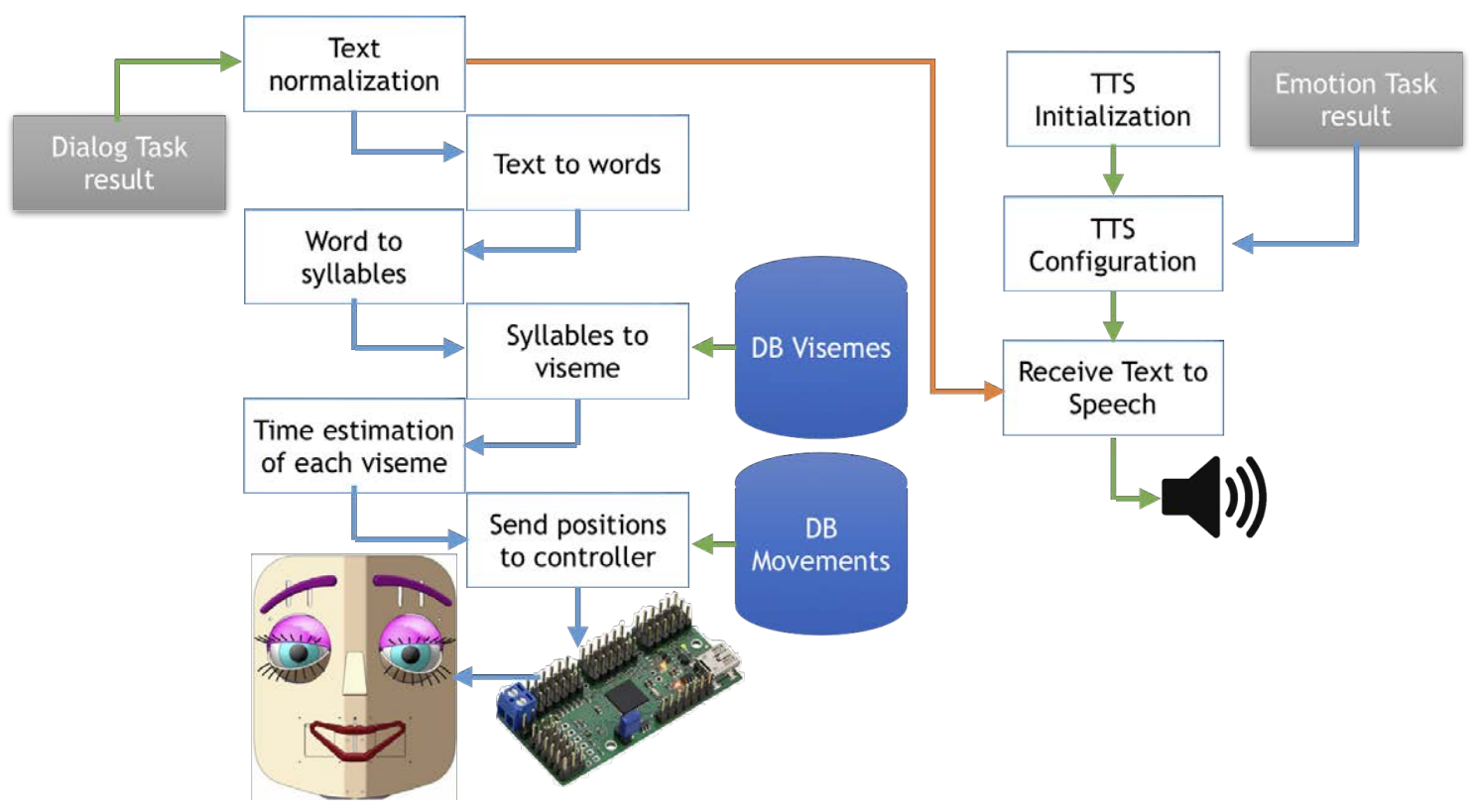

Figure 5.3: Lips synchronization flowchart

Concerning the viseme database, the Spanish language has 27 letters which are combined considering the syllabication rules results in almost 2500 syllables. These syllables where added into into an XML file which its contens is organized in 52 groups. Having the 6 degrees of freedom provided by the Doris mouth, the figure 5.4 shows three different positions, starting from the neutral position 5.4a passing to a viseme representation to pronounce the letter $\mathrm{A} 5.4 \mathrm{~b}$ and the letter $\mathrm{O} 5.4 \mathrm{c}$. 


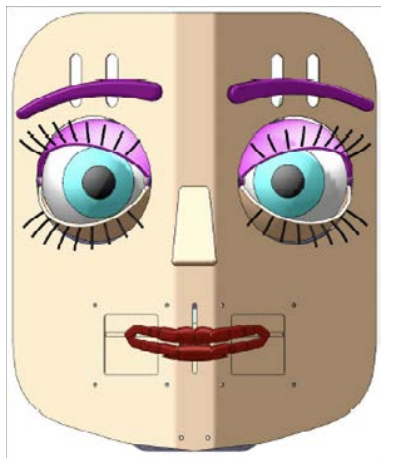

(a) Neutral Position

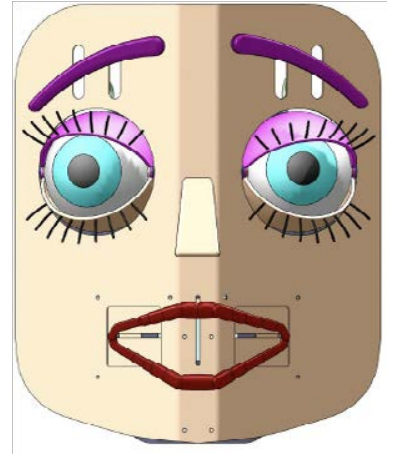

(b) A Viseme

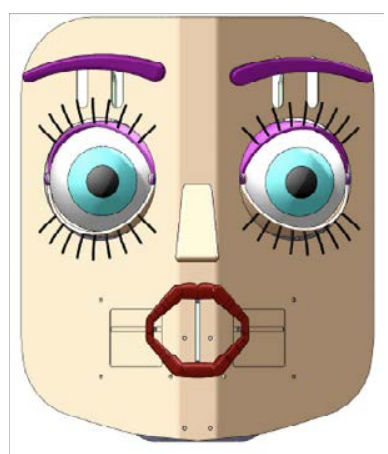

(c) O Viseme

Figure 5.4: Different viseme positions

\subsection{The speaking system}

As Doris has the need to communicate with people, a task for dialoguing has been created. This task receives a text as input provided by the user who she is interacting with and according to the current emotion the task will provide a reasonable answer which is sent to the TTS module responsible for playing the answer. [109] performs a very similar task, being able to interact with several people, a very common issue in places with people as a laboratory or a museum. [110] performs a similar task with the robot NAO which can speak english and sami language. Emotions Task and the TTS module will be explained in sections 5.4 and 5.2 respectively. Figure 5.5 illustrates a brief explanation of how the task operates.

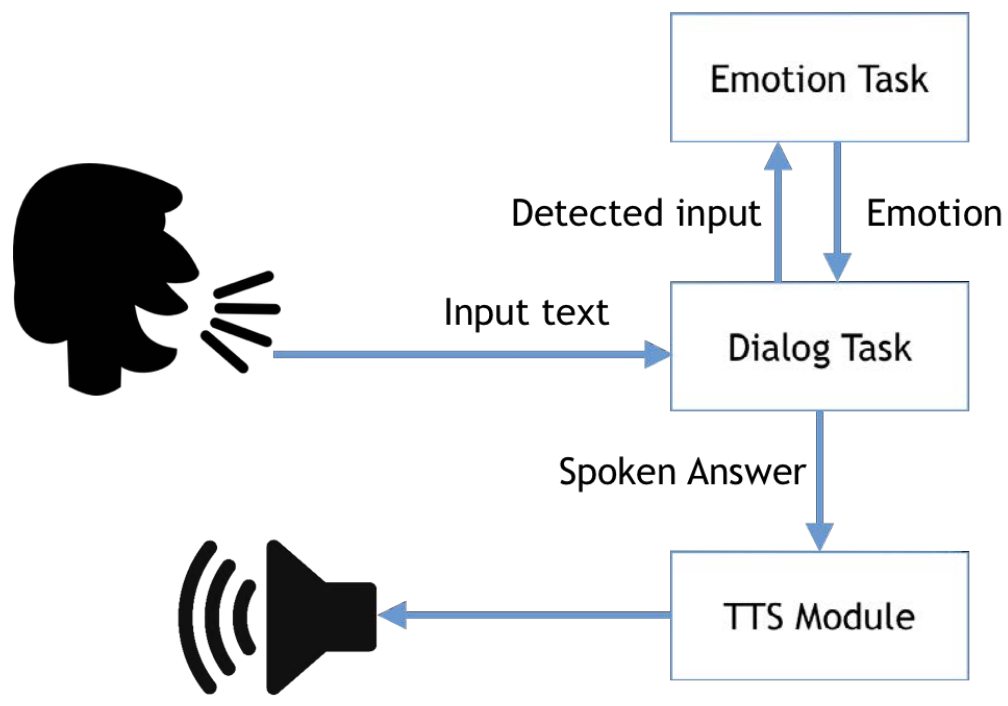

Figure 5.5: Dialog task scheme

As stated, the dialog task receives an input text by the user. This text is analyzed trying to find the same input in the database, or at least the most similar text, which is an 
XML file composed as next:

Listing 5.1: Sample of XML file of input and output messages

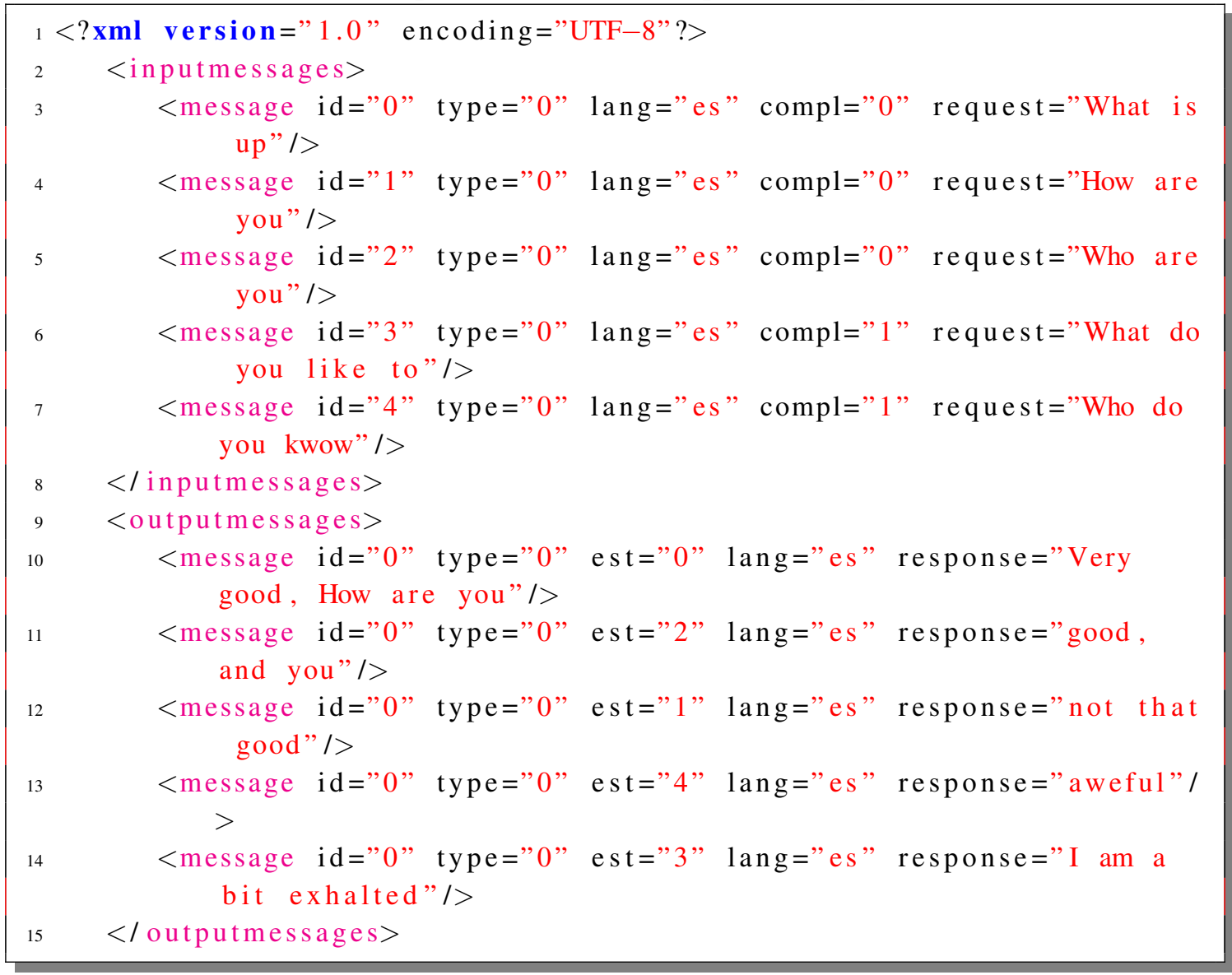

As seen in XML sample file 5.1 there is an attribute named compl in the group of input messages meaning if the phrase is complete (0) or not (1) comparing the input text with the value in the request attribute. The winner request is the one with the highest number of word hits, taking the id of the winner request.

This id is sent to the Emotion Task which will provide the corresponding emotion state. This emotion state is compared with the est attribute located in the output message group which along with the previous provided id, the response is selected. Finally this response is sent to the corresponding TTS module. The task is detailed in the algorithm 2 


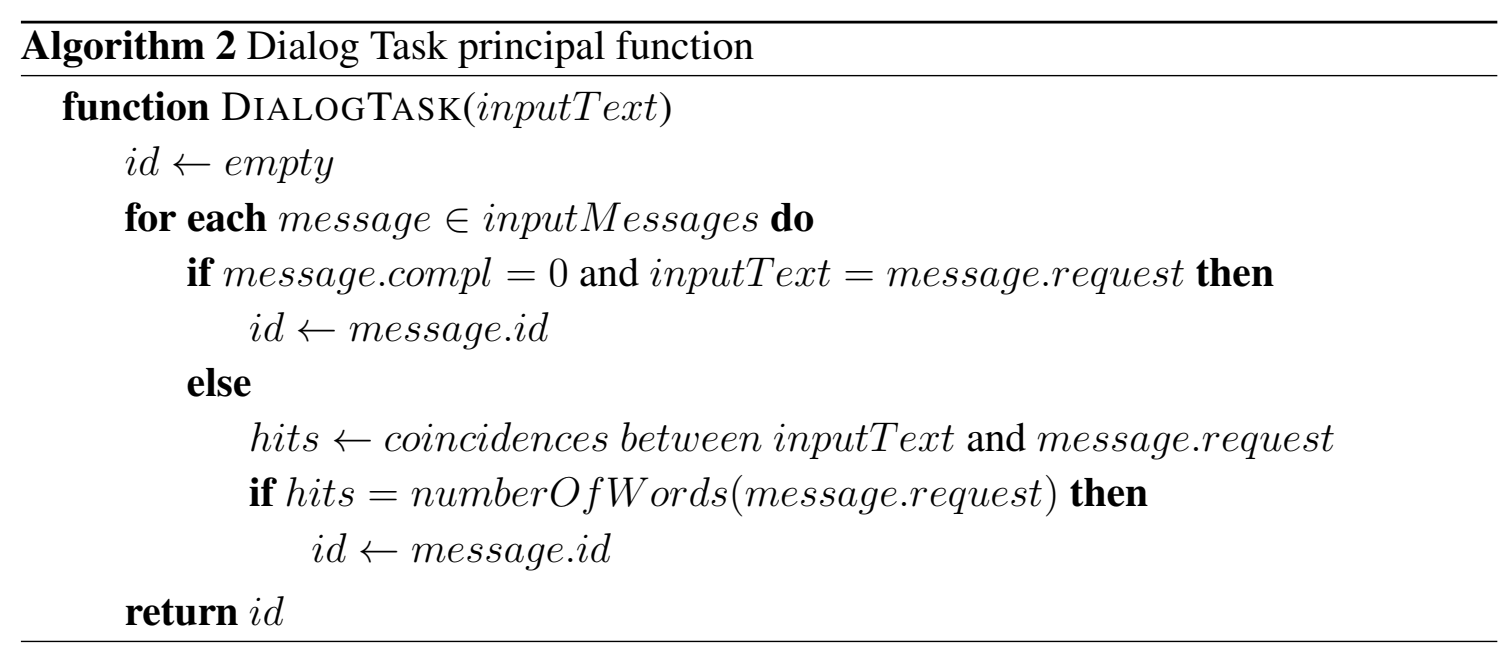

Then when the Emotion Task returns the emotion state, the final response is selected randomly from the output messages set. Algorithm 3

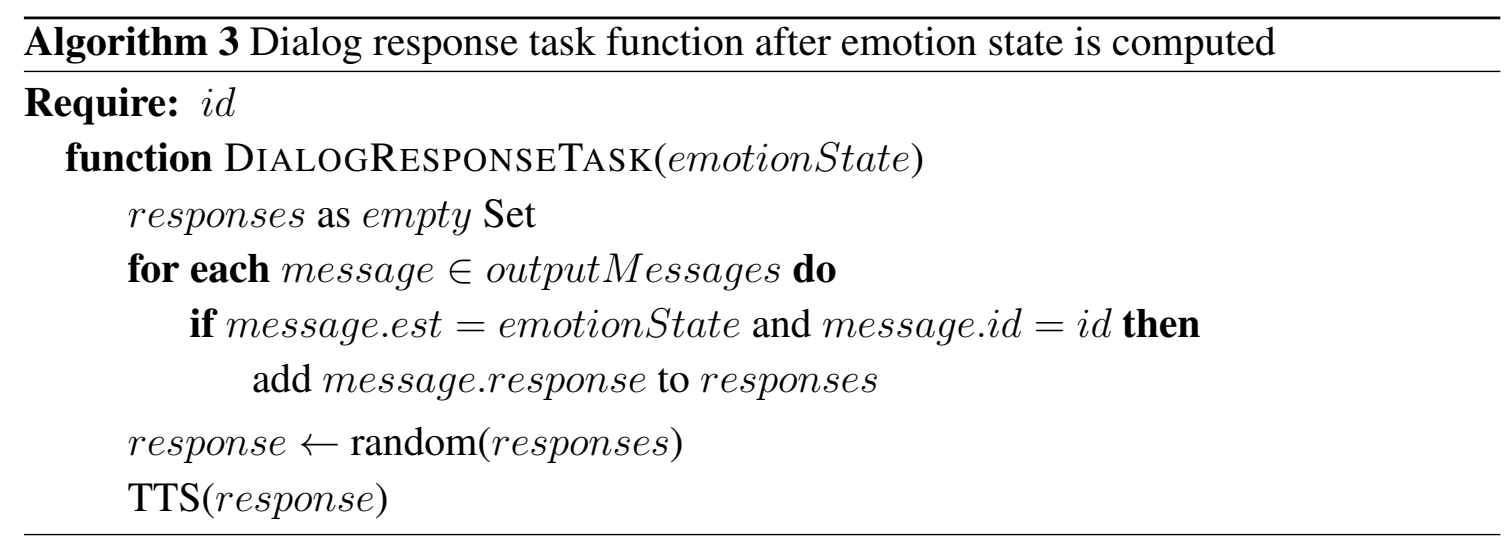

\subsection{The emotion system}

In recent researches, people have tried to go further in robotics trying to develop a system with the ability to provide to the robot a characteristic so that the user does not feel like talking to a simple machine. One of the paths to achieve this characteristic can be by providing the robot with some kind of emotions. [111] proves that designers of human humanoid robots must attend several social cues that most robots do not emit like showing stimuli from different users input. Trying to pursue this emotional robots, can be found iCat in [112] a chess gamer robot that shows its own emotions, which was created by heuristic evaluation of the state of the game. While the iCat is winning his emotions arise as happiness but when loosing the emotions level decrease and show sadness or anger. But now the aim is beyond, not only by showing faces but also to a real feel or awareness of the emotion state, by using a semantic self-representation as described by [113] and [114]. 
As Doris is intended for human interaction, it was required that Doris has a system that allow to change its internal behaviour so it can pretend to establish a more human conversation.

This emotion system consists on the user's formulation of a question, which arrives to Doris as a text string. This string is processed in the dialogs system explained in 5.3 by being looked up in a database where common questions and requests are stored. Depending on the result of this system, an id is provided to the emotion system in order to be matched in the impulses database. This database is shown in listing 5.2 which contains variations how each emotion variables are affected according to the text supplied by the user.

Listing 5.2: Sample of XML emotions variations

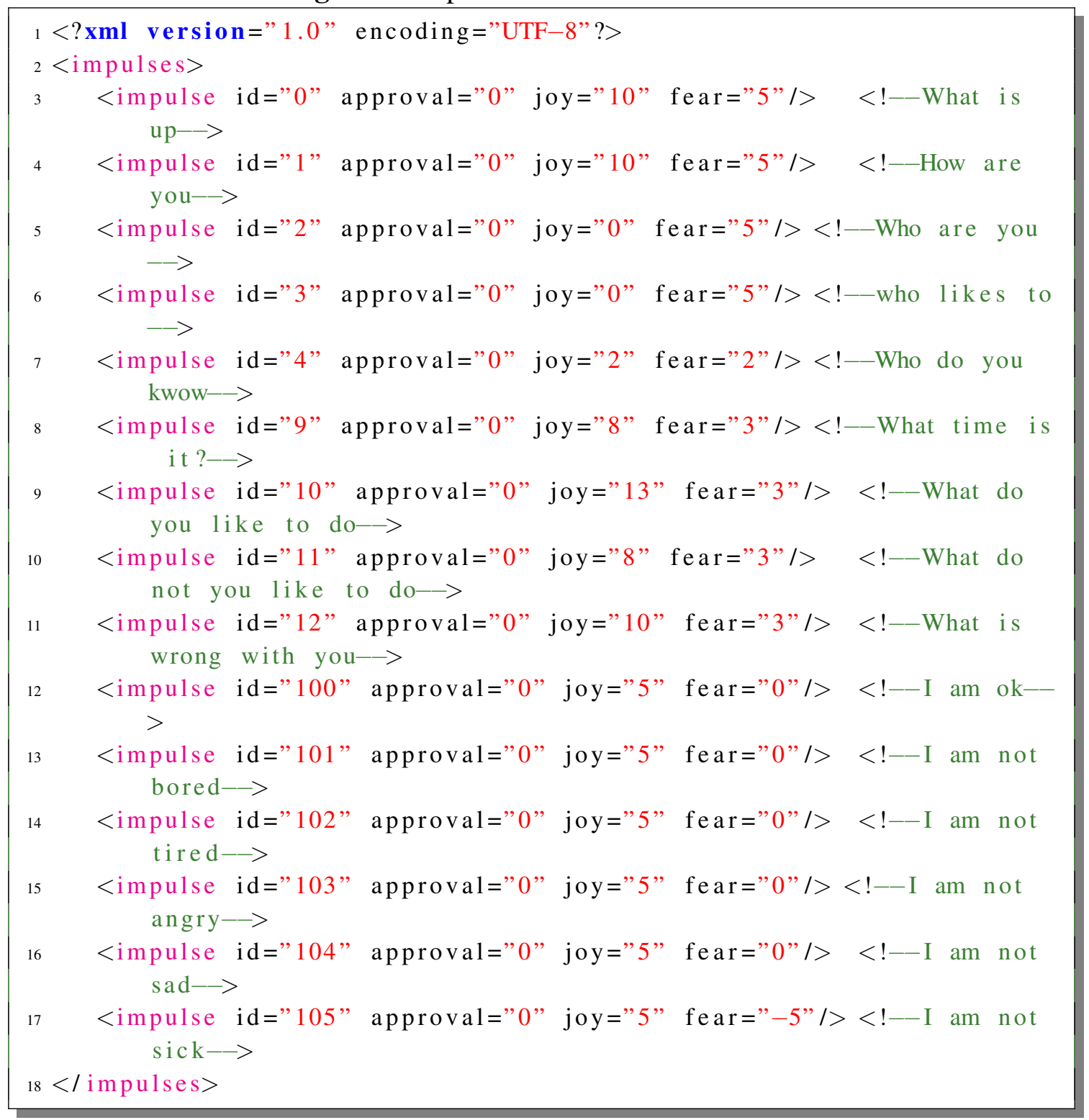

The decision on how each emotion variable varies depending on the input has been taken empirically and according to the previous work developed by [15] and how it is the 
interaction between membership functions of Doris. The set of emotions is based on these state variables with ranges from 0 to 100 :

- Approval: which semantic values are dislike, normal and like.

- Joy: which semantic values are sad, normal and happy.

- Fear: which semantic values are affraid, normal and calm.

According to what has been previously mentioned, the new value of the emotion is computed using the incremental values and adding them to the previous emotions values, and can be expressed as:

$$
\mathbf{x}(k+1)=\mathbf{x}(k)+\Delta \mathbf{x}(k)
$$

Each state variable is bounded to the minimum and maximum values for the emotion system always be stable. Finally to compute the final emotion, the state variables are used as inputs in a Fuzzy Engine. Each variable can be represented as a Fuzzy set shown in figure 5.6, where each membership function was calibrated to each emotion individually according to the desired behaviour for Doris.

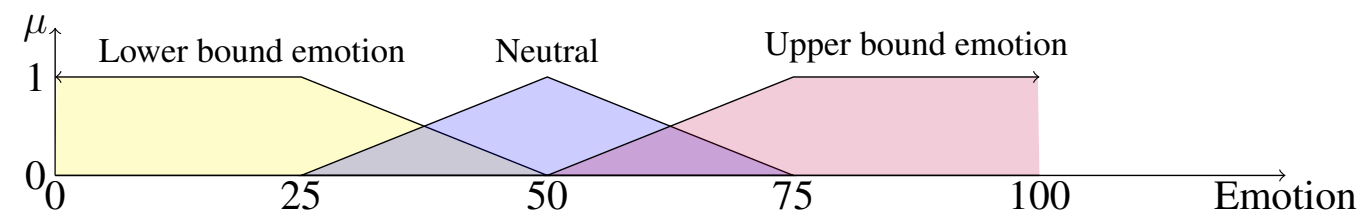

Figure 5.6: Input emotion variables sample as a Fuzzy Set

The fuzzy system outputs are the different level of expressions of Doris face, taking into account the eyelids, eyebrows and mouth positions to express all emotions. The tone and the final output message are also outputs of the fuzzy system:

- Eyelids: which semantic values are closed, half closed, normal, half opened and opened, with a range from 0 o 100.

- Eyebrows: which semantic values are low, Normal and high, with a range from 0 o 100.

- Mouth: which semantic values are so sad, sad, normal, smiley and big smiley, with a range from 0 o 100.

- Voice Rate: which semantic values are low, normal and high, with a range from 0 o 100. 
- Response State: which semantic values are blue, hypocrite, normal, easy and enthusiastic, with a range from 0 o 5 .

The fuzzy outputs are triangular membership functions:

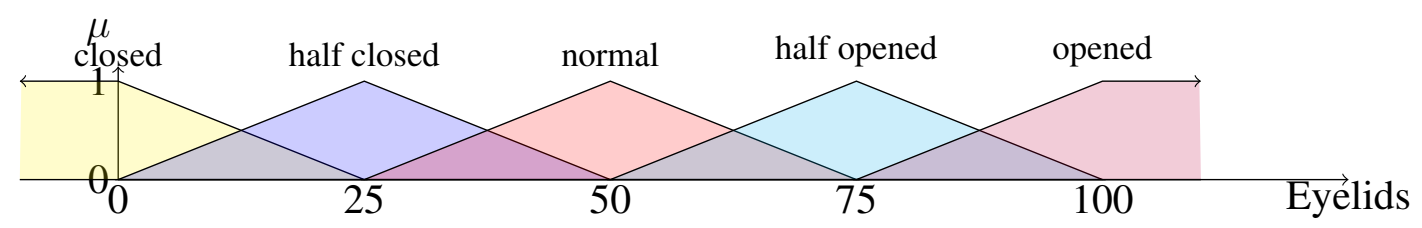

Figure 5.7: Fuzzy output eyelids variable

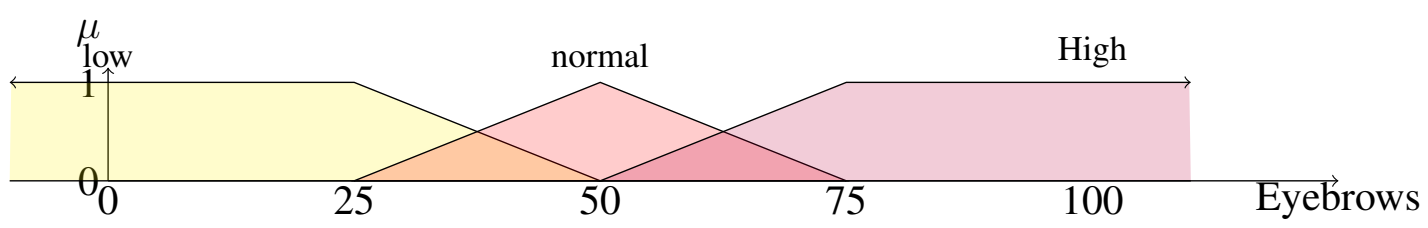

Figure 5.8: Fuzzy output eyebrows variable

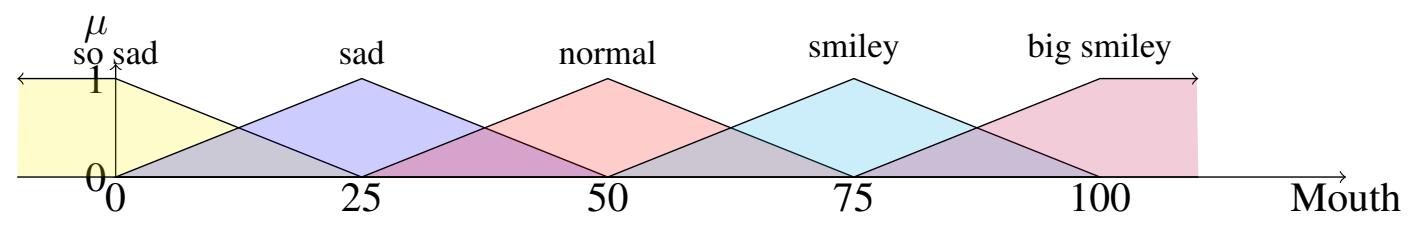

Figure 5.9: Fuzzy output Mouth variable

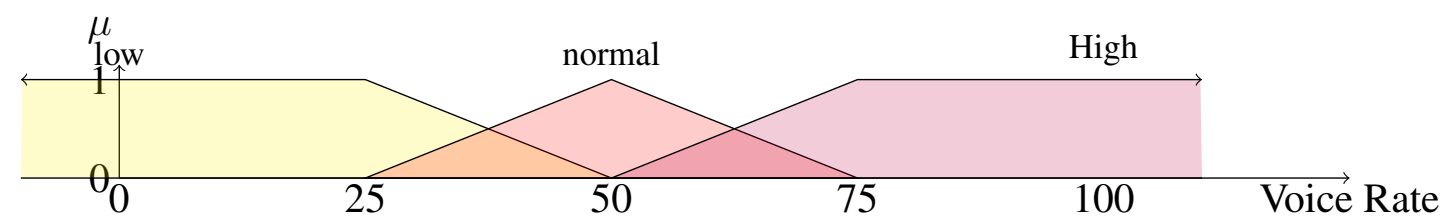

Figure 5.10: Fuzzy output voice rate variable

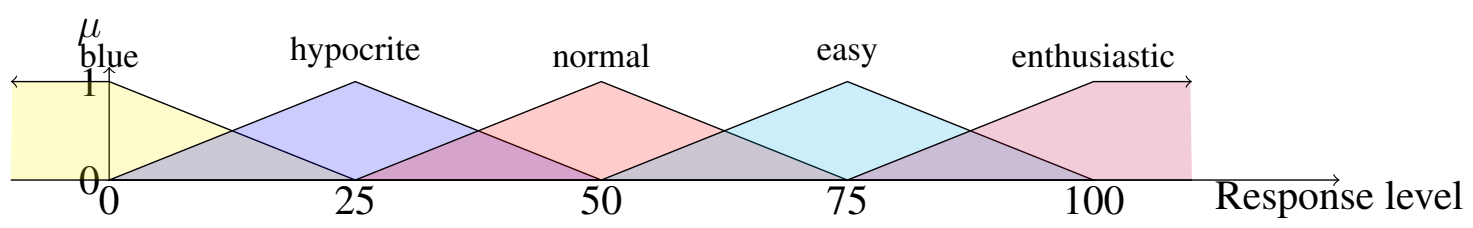

Figure 5.11: Fuzzy output response level variable

As every fuzzy system, it needs a set of fuzzy rules in order to decide which can be the final output value. As the fuzzy system contains 3 input variables with 3 membership functions, by combination it will result in 27 rules. 
Listing 5.3: Emotion system fuzzy rules

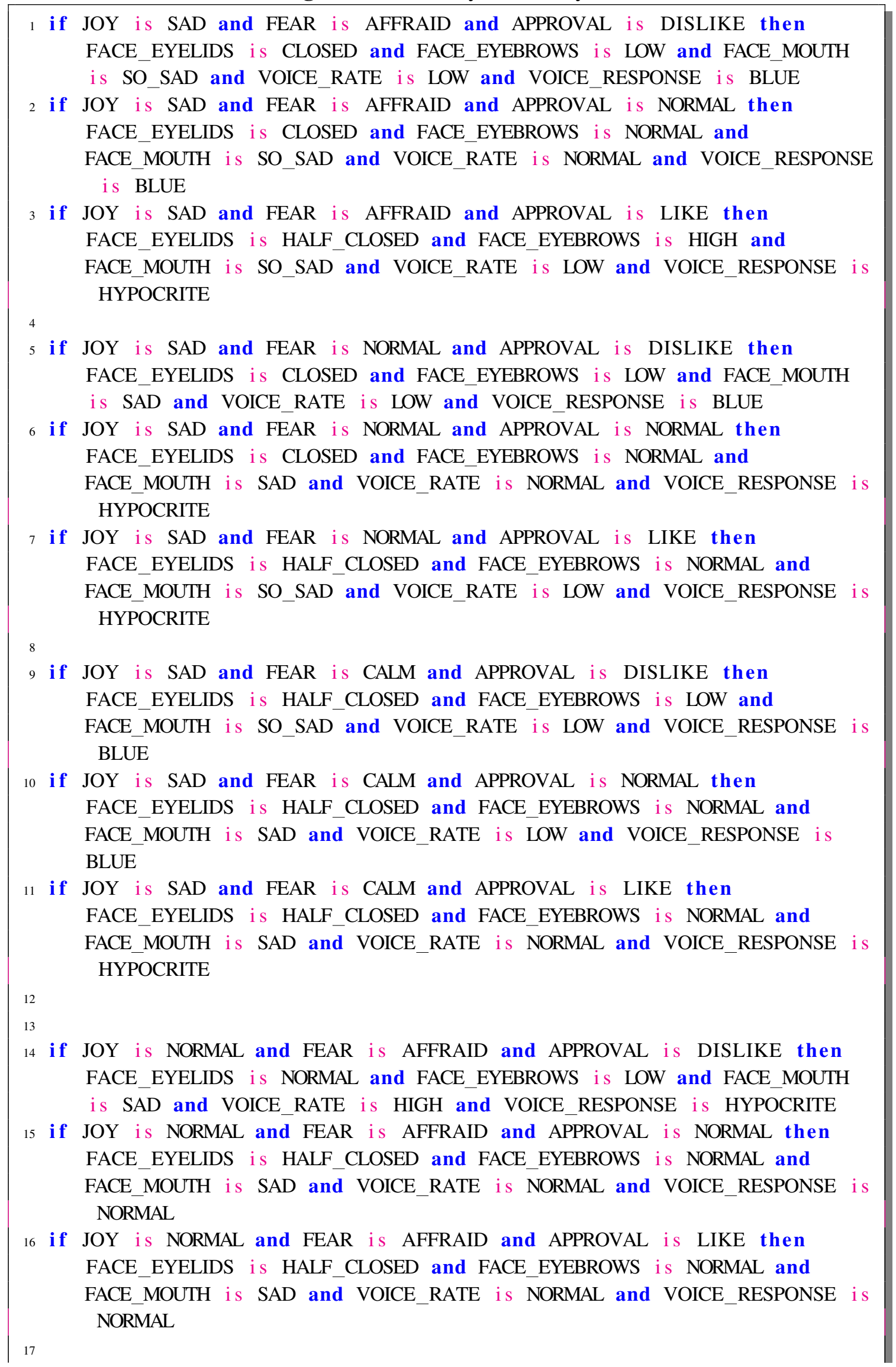


18 if JOY is NORMAL and FEAR is NORMAL and APPROVAL is DISLIKE then FACE_EYELIDS is NORMAL and FACE_EYEBROWS is LOW and FACE_MOUTH

is NORMAL and VOICE_RATE is LOW and VOICE_RESPONSE is HYPOCRITE

19 if JOY is NORMAL and FEAR is NORMAL and APPROVAL is NORMAL then FACE_EYELIDS is NORMAL and FACE_EYEBROWS is NORMAL and

FACE_MOUTH is NORMAL and VOICE_RATE is NORMAL and VOICE_RESPONSE is NORMAL

20 if JOY is NORMAL and FEAR is NORMAL and APPROVAL is LIKE then FACE_EYELIDS is NORMAL and FACE_EYEBROWS is NORMAL and FACE_MOUTH is NORMAL and VOICE_RATE is HIGH and VOICE_RESPONSE is NORMAL

21

22 if JOY is NORMAL and FEAR is CALM and APPROVAL is DISLIKE then FACE_EYELIDS is NORMAL and FACE_EYEBROWS is LOW and FACE_MOUTH

is NORMAL and VOICE_RATE is NORMAL and VOICE_RESPONSE is NORMAL

23 if JOY is NORMAL and FEAR is CALM and APPROVAL is NORMAL then FACE_EYELIDS is NORMAL and FACE_EYEBROWS is HIGH and FACE MOUTH

is NORMAL and VOICE_RATE is NORMAL and VOICE_RESPONSE is EASY

24 if JOY is NORMAL and FEAR is CALM and APPROVAL is LIKE then

FACE_EYELIDS is NORMAL and FACE_EYEBROWS is HIGH and FACE_MOUTH

is SMILEY and VOICE_RATE is NORMAL and VOICE_RESPONSE is EASY

25

26

27 if JOY is HAPPY and FEAR is AFFRAID and APPROVAL is DISLIKE then FACE_EYELIDS is HALF_OPENED and FACE_EYEBROWS is NORMAL and FACE_MOUTH is SAD and VOICE_RATE is LOW and VOICE_RESPONSE is HYPOCRITE

28 if JOY is HAPPY and FEAR is AFFRAID and APPROVAL is NORMAL then FACE_EYELIDS is HALF_OPENED and FACE_EYEBROWS is NORMAL and FACE_MOUTH is NORMAL and VOICE_RATE is LOW and VOICE_RESPONSE is NORMAL

29 if JOY is HAPPY and FEAR is AFFRAID and APPROVAL is LIKE then FACE_EYELIDS is HALF_OPENED and FACE_EYEBROWS is NORMAL and FACE_MOUTH is NORMAL and VOICE_RATE is NORMAL and VOICE_RESPONSE is EASY

30

31 if JOY is HAPPY and FEAR is NORMAL and APPROVAL is DISLIKE then FACE_EYELIDS is HALF_OPENED and FACE_EYEBROWS is NORMAL and FACE_MOUTH is SAD and VOICE_RATE is NORMAL and VOICE_RESPONSE is NORMAL

32 if JOY is HAPPY and FEAR is NORMAL and APPROVAL is NORMAL then FACE_EYELIDS is HALF_OPENED and FACE_EYEBROWS is HIGH and FACE_MOUTH is SMILEY and VOICE_RATE is NORMAL and VOICE_RESPONSE is EASY

33 if JOY is HAPPY and FEAR is NORMAL and APPROVAL is LIKE then FACE_EYELIDS is HALF_OPENED and FACE_EYEBROWS is HIGH and FACE_MOUTH is SMILEY and VOICE_RATE is HIGH and VOICE_RESPONSE is EASY

34

36 if JOY is HAPPY and FEAR is CALM and APPROVAL is DISLIKE then FACE_EYELIDS is OPENED and FACE_EYEBROWS is LOW and FACE_MOUTH 
is SMILEY and VOICE_RATE is NORMAL and VOICE_RESPONSE is EASY

37 if JOY is HAPPY and FEAR is CALM and APPROVAL is NORMAL then

FACE_EYELIDS is OPENED and FACE_EYEBROWS is NORMAL and

FACE_MOUTH is SMILEY and VOICE_RATE is NORMAL and VOICE_RESPONSE

is EASY

38 if JOY is HAPPY and FEAR is CALM and APPROVAL is LIKE then

FACE_EYELIDS is OPENED and FACE_EYEBROWS is HIGH and FACE_MOUTH

is BIG_SMILEY and VOICE_RATE is HIGH and VOICE_RESPONSE is ENTHUSIASTIC

In this set of rules were included hypothetical cases where maybe 2 or 3 emotions can be activated at the same time. for example, a case where a museum visitor is lost and when the person reappears Doris can show happiness and dislikeness at the same time.

\subsection{Robotic arm}

Another form of interaction based in human corporal expression can be using arm based expressions. The influence of the body language in the social abilities, might generate empathy or can also generate distrust or even rejection to other people. This might be due the contradiction between the verbal communication and the corporal language.

So, body language can be understood as a form of communication using gestures, positions and movements of the body to transmit information about emotions and thoughts from the entity who emits them. In the case of Doris, this characteristic body language has been trying to be implemented. Although an arm has been built, it has not been attached to the body due to the motors have a high current consumption.

As the robot must perform autonomously and the arm has not been attached yet to the body, the arm only operates in manual mode. For this reason, it has not been added to the emotional system or the task planner system.

As the arm operates only in manual mode, some predefined expressions has been added to a database, which contains the positions of certain gestures. These gestures are indicate, well-done, piss-off, victory, neutral, walking, one, two, three, salute and handshaking. As these movements are predefined in a database, the user can select the arm position from the database. One the arm position is selected, an id identifying the arm position is sent to Doris, which perform a search algorithm as shown in algorithm 4. 


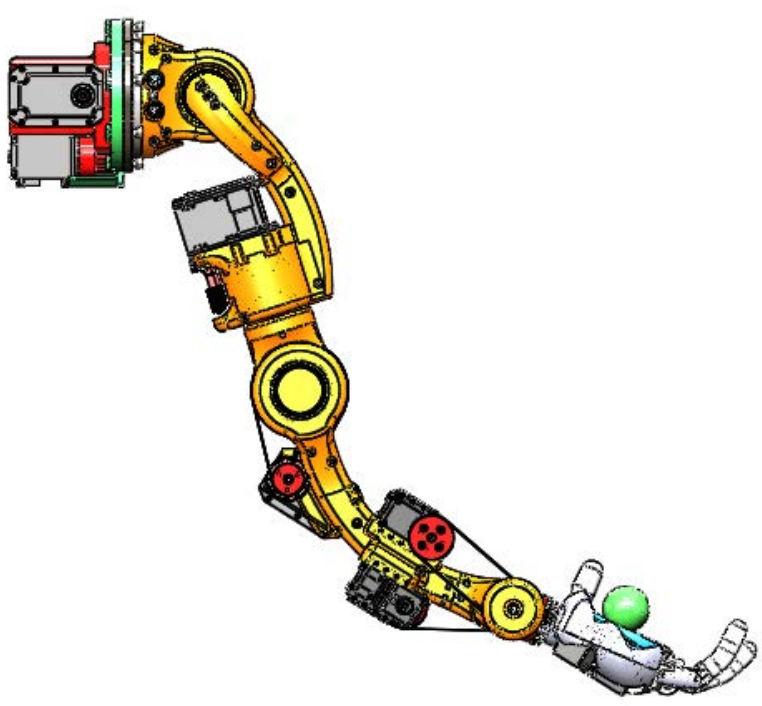

(a) Robotic arm design

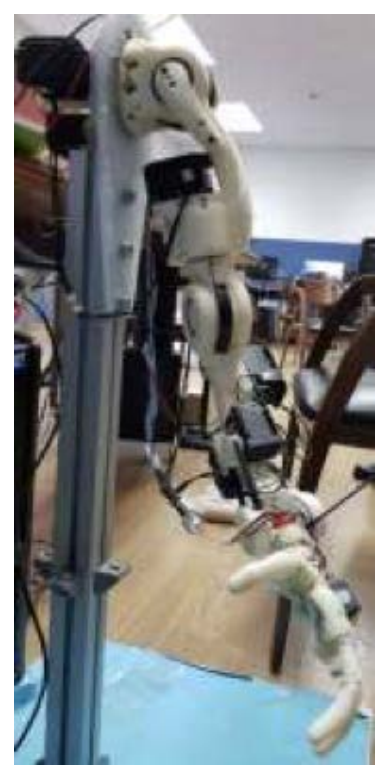

(b) Real view of the arm

Figure 5.12: Doris robotic arm

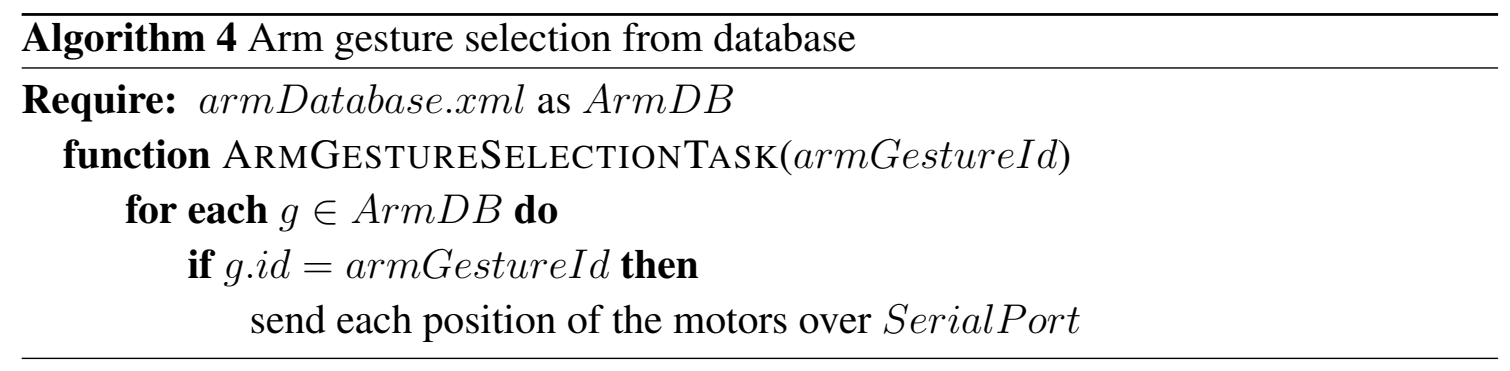

\subsection{Experiments and results}

For the interaction system, the lip sync, the face interaction and the emotion system were tested at the same time by providing some texts as inputs to observe how the emotions vary and how the answers of Doris are randomly selected according to the emotion computed in that moment.

One of the test was to make Doris to get Sad and to achieve this behaviour a set of phrases was sent to the robot. Next table 5.3 shows the change of the emotion on each phrase:

The results shown in the table 5.3 are as expected. Negative input texts to the robot make emotion state change from normal to hypocrite and finally to sad. In the table also can be seen the evolution of each emotion variable, joy and approval variables decrease as expected but Fear almost keeps the same. 
Table 5.3: Doris trying to get Sad

\begin{tabular}{ll|ccc|cc}
\hline $\begin{array}{l}\text { Input } \\
\text { Phrase }\end{array}$ & $\begin{array}{l}\text { Output } \\
\text { Phrase }\end{array}$ & Joy & Fear & Approval & Expression & Emotion Value \\
\hline $\begin{array}{l}\text { You are a } \\
\text { fool }\end{array}$ & $\begin{array}{l}\text { Do not say } \\
\text { that to me }\end{array}$ & 37 & 47 & 40 & $\begin{array}{c}\text { Normal } \\
\text { Hypocrite }\end{array}$ & 1 \\
$\begin{array}{l}\text { You are } \\
\text { you are } \\
\text { joking }\end{array}$ & 32 & 50 & 30 & Sad & 0 \\
$\begin{array}{l}\text { You are } \\
\text { do not say } \\
\text { dupid }\end{array}$ & 27 & 55 & 20 & So sad & 0 \\
Jackanapes & $\begin{array}{l}\text { you have } \\
\text { gone crazy }\end{array}$ & 22 & 58 & 10 & So sad & 0 \\
\hline
\end{tabular}

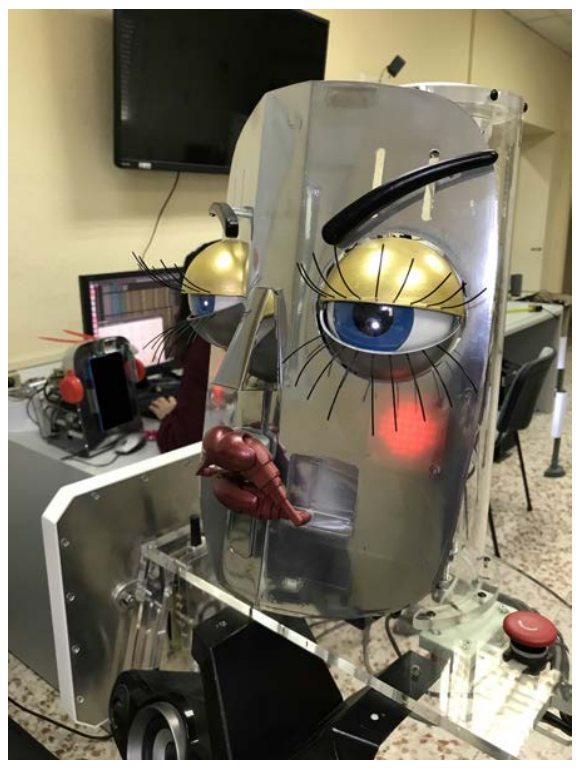

Figure 5.13: Doris getting angry

Another experiment is to try to have a conversation with Doris. As it is assumed, Doris will not win a Turing test but she is not intended to do it. 
Table 5.4: Doris trying to get angry

\begin{tabular}{|c|c|c|c|c|c|c|}
\hline $\begin{array}{l}\text { Input } \\
\text { Phrase }\end{array}$ & $\begin{array}{l}\text { Output } \\
\text { phrase }\end{array}$ & Joy & Fear & Approval & Expression & Emotion Value \\
\hline & & 50 & 50 & 50 & Normal & 2 \\
\hline $\mathrm{Hi}$ & $\mathrm{Hi}$ & 60 & 55 & 50 & Normal & 3 \\
\hline $\begin{array}{l}\text { Do you } \\
\text { admire } \\
\text { somebody? }\end{array}$ & $\begin{array}{l}\text { I admire } \\
\text { Biel, and } \\
\text { you? }\end{array}$ & 70 & 60 & 50 & Bit Happy & 3 \\
\hline $\begin{array}{l}\text { What } \\
\text { is your } \\
\text { favourite } \\
\text { song? }\end{array}$ & $\begin{array}{l}\text { Don't stop } \\
\text { me now by } \\
\text { Queen }\end{array}$ & 72 & 62 & 50 & Bit Happy & 3 \\
\hline $\begin{array}{l}\text { And your } \\
\text { favourite } \\
\text { movie? }\end{array}$ & Matrix & 87 & 67 & 50 & Bit Happy & 3 \\
\hline $\begin{array}{l}\text { You are so } \\
\text { funny }\end{array}$ & Thank you & 92 & 72 & 55 Нарру & 3 & \\
\hline Nucklehead & $\begin{array}{l}\text { Have you } \\
\text { lost your } \\
\text { mind? }\end{array}$ & 87 & 75 & 45 & Less Happy & 3 \\
\hline $\begin{array}{l}\text { You are } \\
\text { clumsy }\end{array}$ & $\begin{array}{l}\text { Why do } \\
\text { you say } \\
\text { that? }\end{array}$ & 74 & 72 & 35 & Less Happy & 2 \\
\hline $\begin{array}{l}\text { You are } \\
\text { fool }\end{array}$ & $\begin{array}{l}\text { Please, } \\
\text { don't say } \\
\text { that again }\end{array}$ & 68 & 66 & 25 & Bit Sad & 2 \\
\hline $\begin{array}{l}\text { You are } \\
\text { fool }\end{array}$ & $\begin{array}{l}\text { Please, } \\
\text { don't insist } \\
\text { with the } \\
\text { same }\end{array}$ & 57 & 60 & 14 & Bit Angry & 1 \\
\hline
\end{tabular}

Some adjustments needed to be performed in order to achieve a better performance and the emotion system do not move from one emotion to other drastically. These adjustments were made in the impulses xml file shown in listing 5.2

A full video, named Emotion System Test: Setting Doris Sad, where the robot is performing a set of text inputs provided by a user can be found at the group's webpage gallery Intelligent Control Group UPM-CSIC - Gallery 


\subsection{Conclusions}

People can react differently when dealing with the same task, hence, interaction in robots is hard to achieve. So making a robot that can interact with different types of audiences is not easy. Despite this, the goal, which consisted in Doris' interaction with environment and the avoidance of the uncanny valley, was achieved. Doris' physical appearance is very well accepted among people.

An emotion system has also been developed in order to achieve a more realistic interaction. The system modifies the face and the voice and provides an output message to the visitor according to the emotion at that instant.

The use of a fuzzy engine to show emotion in the face and also in the voice is very useful to achieve a gradual evolution of each emotion input. Other emotion variables will be taken into account for future developments. 


\section{Teleoperation system}

The remote control of Doris is another proposal of this dissertation. This control is performed via ethernet connection being it possible to operate the robot in a local area network or in a wide area network, so that a robot located in a museum in Italy can be operated by a person located in Spain. This was the most important reason for a client-server architecture to be chosen in the first place.

The client-server architecture is a type of application where the information is distributed throughout several systems called servers and the requesters of that information are called clients. Under this scope, Doris is the only server, but how many and what type of clients can the robot handle? In this chapter, the process of developing client applications to communicate and operate the robot will be explained. The applications can be developed for desktop or mobile devices.

The client is an application which performs a request to the server. This information travels through a communication tunnel, normally ethernet, and then the server returns the information requested to the user. For example, when a webpage is visited, a HTTP request is submitted to a server, then the server responds with the content of the webpage.

As proposed in chapter 3, the architecture should handle different clients, like clients connected with a smartphone or tablet and clients connected via web. As the applications will connect to Doris over ethernet, sockets are used except for web applications, where web-sockets are used instead. A web-socket is just an ordinary socket but working with the security protocol SHA1 (Secure Hash Algorithm).

The design of the client applications is significant in its development. The userinterface must be user-friendly and not overloaded with buttons or animations. All the information given throughout this chapter is key to this dissertation because it is the first step to connect and begin with the operation of Doris.

In this chapter, the initial considerations of the applications are shown, explaining 
what the applications must contain to have a fully remote control of the robot. The design of each application is considered subsequently, centring on the android and web applications. Later on, the implementation of each application is given and explained. Finally, some tests and results are shown along with the conclusions.

\subsection{Initial considerations and analysis}

As it has been stated, Doris has been designed for human interaction, but also to interact with the programmer of the robot, who must have remote control and supervision of Doris. Thus, an android and a web applications have been created.

The remote control involves controlling Doris from another room, location, city or even a country, and the communications will work faster depending on the location of the robot supervisor. This web application can work either in mobiles/tablets Android or iOS or in desktop navigators as Mozilla, Safari, Chrome, etc. Similar works can be found at [115] where the authors propose an empathic web interface to configure personal robots. Moreover, in [116] a web interface is developed to control and configure a tour-guide robot with several users.

In this section considerations for the design of the applications will be exposed. Taking into account that it is not the same interface for different devices and the distribution must be adapted to each device. It is desired that the interface has a modern and user friendly design.

Nowadays, there are many web libraries that help to develop these type of web applications. These libraries are based on CSS (Cascade Style Sheet) which combined with the power provided by HTML5 and Javascript provide very attractive web applications accepted by users. Bootstrap, AngularJS and others are the most common libraries for design.

Concerning android mobile devices, it is possible to develop applications using $\mathbf{Q t}$ which is a software development kit (SDK) using $\mathrm{C}++$ with the capacity to generate Android or iOS applications.

Aside from the design, the application must handle information provided by the robot and also send requests to the server and then receive the respective answers also from the robot. Based on this main characteristics the all the applications should contain as follows:

- Robot control owner: The application must contain a button to request to the robot the permission to be the owner and operate it. This ownership allows the user to modify the expression of the robot, move the robot to another point, load the actions program that commands Doris to perform a tour in a museum and 
others. If the robot is being operated by another user and a request of permission is performed, a pop-up window should appear to the current user accepting or rejecting the ownership request.

- Face expressions: as it was stated, the web application should modify current robot face expression. A tab must be created to perform this action. This tab must also include a button that allows to send an input text to Doris as a request. for example, asking about what time is it, or what her name is, etc.

- Navigation: this section must be a tab inside the web page that allows the user to load a map being able to observe the distribution of objects and points of interest (sites) in the area. This tab must also include to move the robot manually or to load an action script selected from a list.

- Emotions: this tab can only be used to observe the evolution of the emotions from Doris. The evolution will be shown in charts.

- Arm expressions: just like the face expressions section, a tab is created to operate manually the robotic arm for doris.

- Settings: a tab allowing the user to change the default configuration values such as, the robot's speed, connection settings, etc.

The use case diagram shown in figure 6.1 explains how the interaction is performed between the user and Doris. It can be seen that there is a full connection between the user and the robot through the application except for the setting section that this only modifies local configuration.

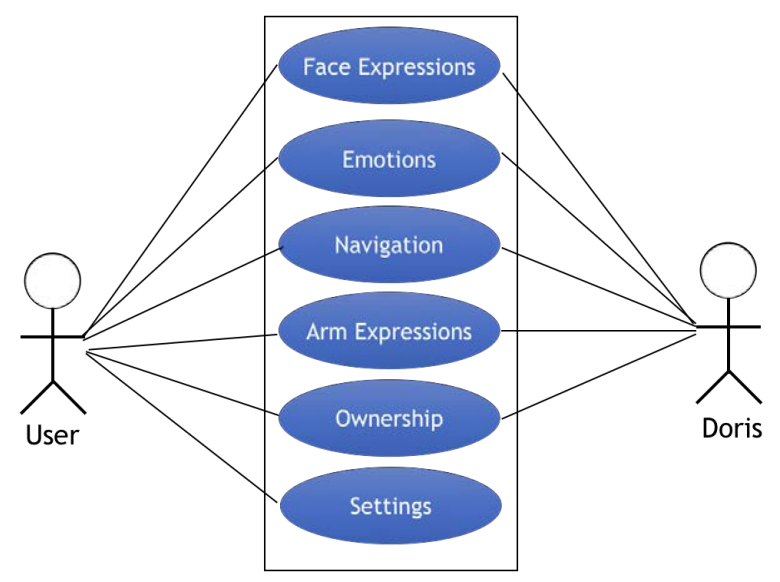

Figure 6.1: Use case diagram of client application

Each one of the use cases in the 6.1 is detailed above. Concerning the face controller use case, the figure 6.2 also shows the interaction between the user and Doris. The use case shows the need of a component that can list all the faces that Doris can display and 
listed in the chapter 5 and also a button to show a dialog and send an input text. In this dissertation the input text can be obtained by means of a text dialog or by using a voice recognizer available in the mobile device or computer.

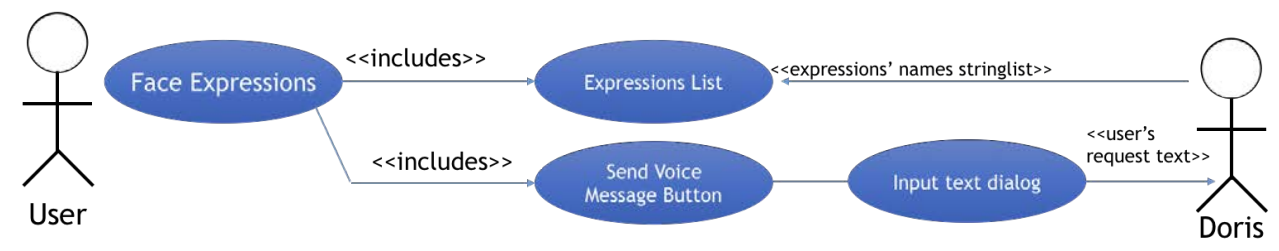

Figure 6.2: Use case diagram of face expression section

The emotions use case can also be found in the client application requirements. As stated in the description, this section will only show Doris' emotions evolution in charts. Figure 6.3 shows the relationship between the user and Doris.

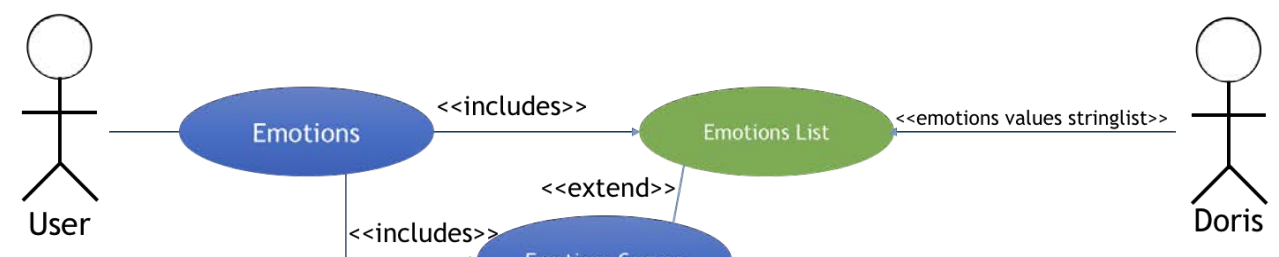

Figure 6.3: Use case diagram of emotions section

In figure 6.4 the navigation use case is shown. When the navigation tab is selected, the map list is loaded, the map list contains each of the sectors present in the respective map, and each sector contains the respective sites and features as explained in the semantic map section in chapter 4. The module can also switch between the manual and autonomous navigation mode.

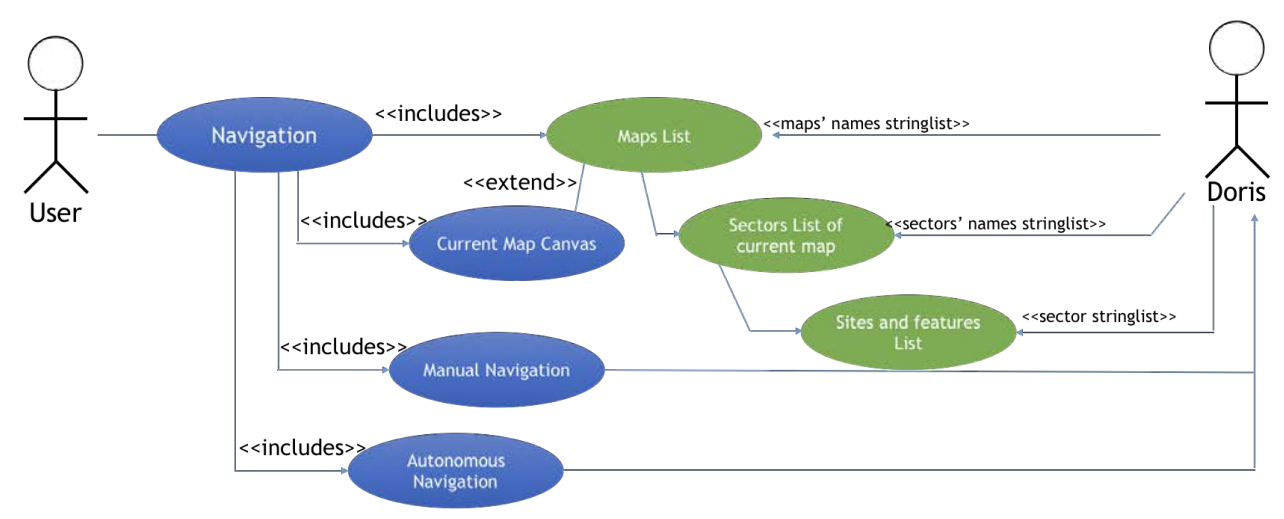

Figure 6.4: Use case diagram of the navigation section

Like the face controller use case, the arm controller use case shown in figure 6.5 will have the same functionality, the arm expressions will be shown in a list so the user can 
select one of the available expressions. The tab will also show several sliders, each one for each motor in the robotic arm.

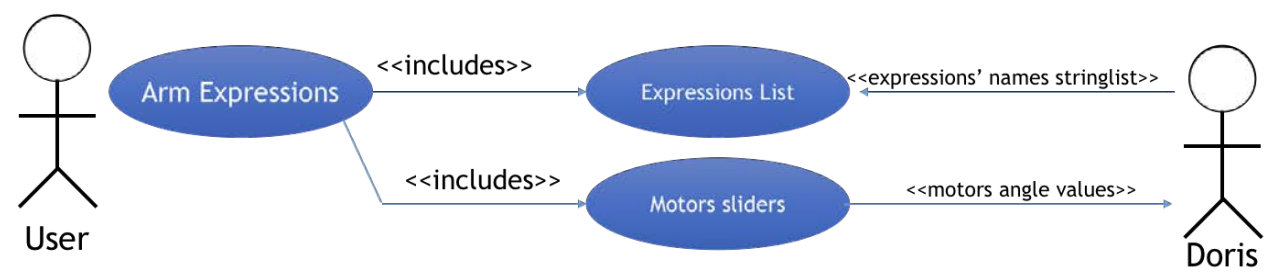

Figure 6.5: Use case diagram of arm expression section

Finally the ownership use case, referencing the user who is able to control the robot in that current moment. It will be a simple button requesting the permission to handle the robot and if it is available, the ownership will be granted to that user. If the control of the robot is owned by another user, then the server sends the request to the current robot owner waiting ten seconds for a response to grant or reject the permission to the user. If after 10 seconds the current robot owner does not answer, the request of using Doris is denied.

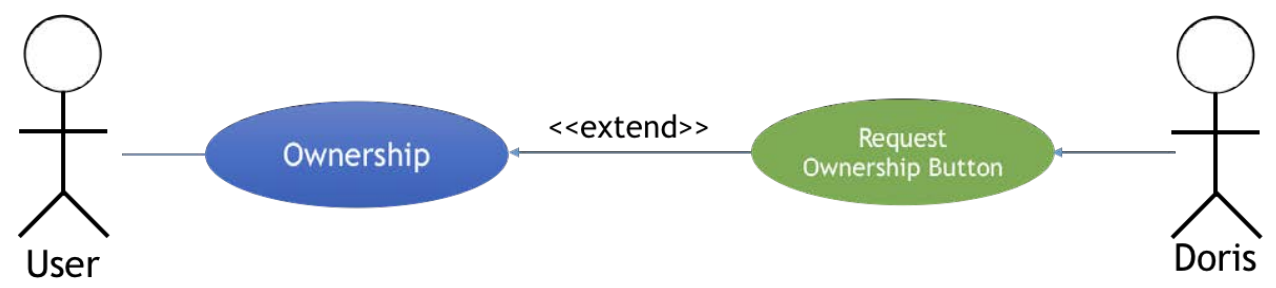

Figure 6.6: Use case diagram of requesting robot ownership

\subsection{Design}

In this section, the software solution to the problems stated in the requirements section with its respective methodology and design will be introduced. Each component of the software will be defined and translated into class diagrams for both applications and also some extra functionalities are detailed for the web application.

Java or $\mathrm{C}++$ can be used as tools to develop android applications. But both languages are based on the object-oriented paradigm. For this reason, class diagrams were created for each application module. In figure 6.7 the MainActivity class is connected with each application module. The MainActivity class is the first interface displayed in the application, the responsible for showing/hiding the main menu and the responsible for instantiating the other objects present in the menu. 


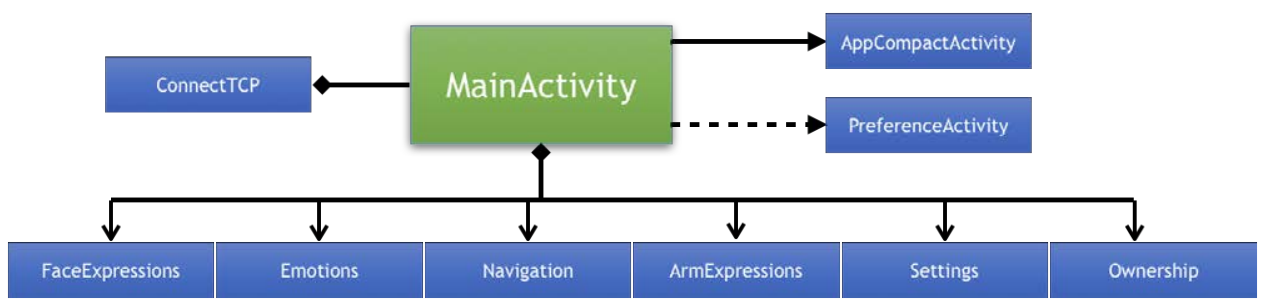

Figure 6.7: Main activity class diagram

In this activity, it is intended that the user navigates through the options in an easy and quick form, so a button will be placed in this activity in order to access to this menu. Besides, the figure shows the need for a connection to the robot to be performed. The ConnectTCP class is the responsible to achieve this connection. In figure 6.8, the structure of the class diagram that it is an implementation of the iSocketNode class that contains all the attributes and methods available to connect to the robot over a normal socket can be seen.

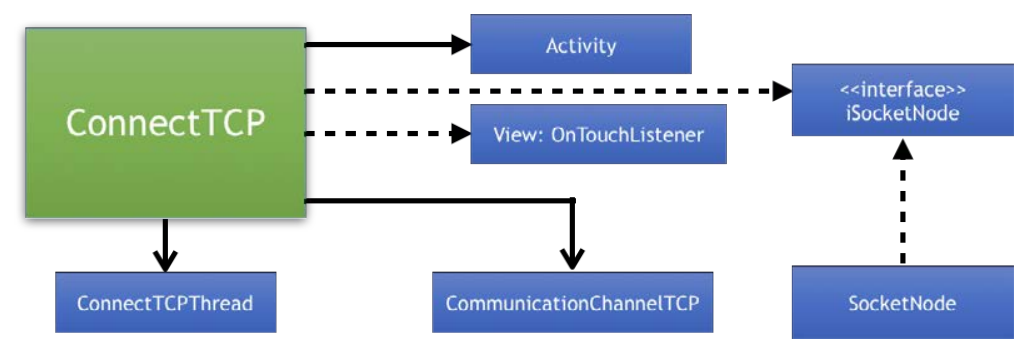

Figure 6.8: TCP client connection class diagram

The class ConnectTCP is the responsible for the exchange of messages with Doris, so the object that instantiate this class must be present along the whole application. This can be performed by creating a singleton object and this being of universal access in the whole application, but the creation of singleton classes are not recommended. Another method consists in passing this object over the different classes using the constructor. An example of this use of ConnectTCP instance can be seen in figure 6.9.

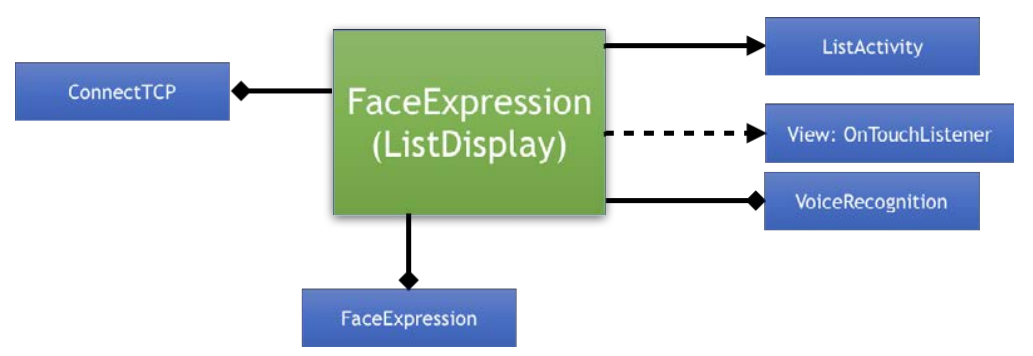

Figure 6.9: Facial expressions class diagram

As specified in the requirements section, it can also be seen that the FaceExpression (ListDisplay) is a class that shows the available faces that Doris can handle. The list 
depends on the FaceExpression class which contains the elements of the face like id, name and picture. This class also has a button which activates the VoiceRecognition that will be used for interacting with Doris.

Navigation is another class presented for this client application which is the commissioned for navigation and mapping purposes shown in figure 6.10. The most relevant classes are the ManualNavigation class (figure 6.12) and the AutonomousNav class (figure 6.11).

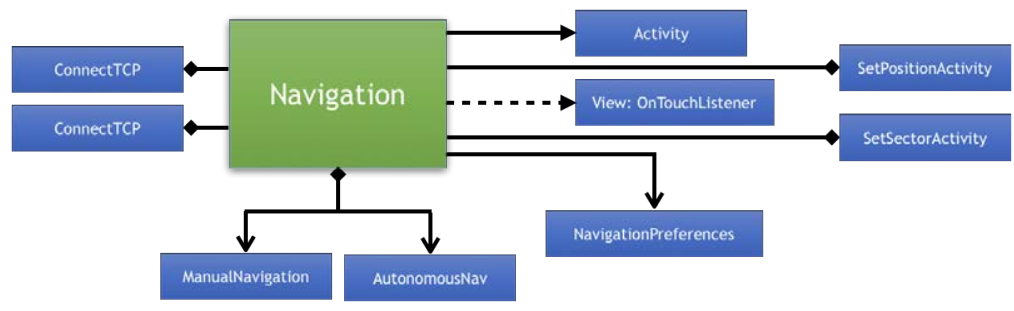

Figure 6.10: Navigation class diagram

The AutonomousNav class shows a program list provided by Doris and when onTouch () event occurs over one of the list items, it will be loaded so that the task planner can be ready to start the tour at the museum.

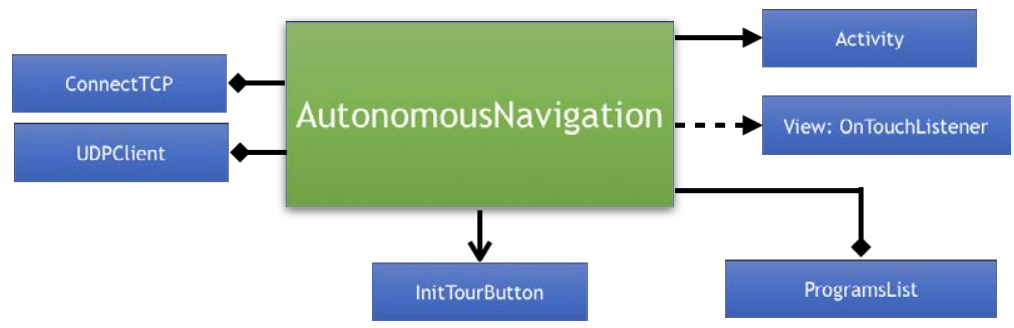

Figure 6.11: Autonomous navigation class diagram

The ManualNavigation class will display two controls, one control allows to move the robot manually forward and backwards and another control that allows the robot to spin manually to the left or to the right, always taking into account the navigation preferences defined by the user.

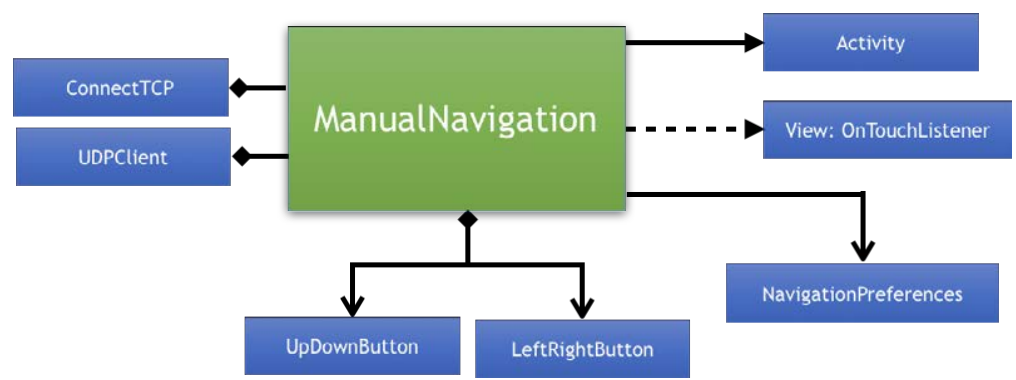

Figure 6.12: Manual navigation class diagram 
The classes defined previously can also be used to develop the web application, specially those components used to display the information. As the application will be developed in JavaScript, it is not possible to model new objects though the language has its own objects, Hence, patterns to model the web application will be defined in this section. The application is subdivided in the following modules:

- Connection: Send and receive messages.

- Pages management: view controllers and pages to load.

- Functional sections: expressions, navigation, etc.

It is important to emphasize that this modular division generates the following files:

- Web pages (.html)

- Javascript files (.js)

- Cascade Style Sheets (.css)

- Other: images, libraries, etc.

The connection with Doris will be performed once the web page is loaded. While loading the webpage, an instance of a javascript object named WebSocket is created and this objects generates four events:

- onopen(evt): this event is launched when the web-socket has successfully connected with the server.

- onclose(evt): this event is launched when the web-socket is closed by the user (closes the webpage) or by an internal error during transmission/reception.

- onerror(evt): this event occurs when the web-socket could not establish the communication with the server

- onmessage(evt): this event is launched when successfully connected and a message arrives to the websocket over the opened tunnel. 


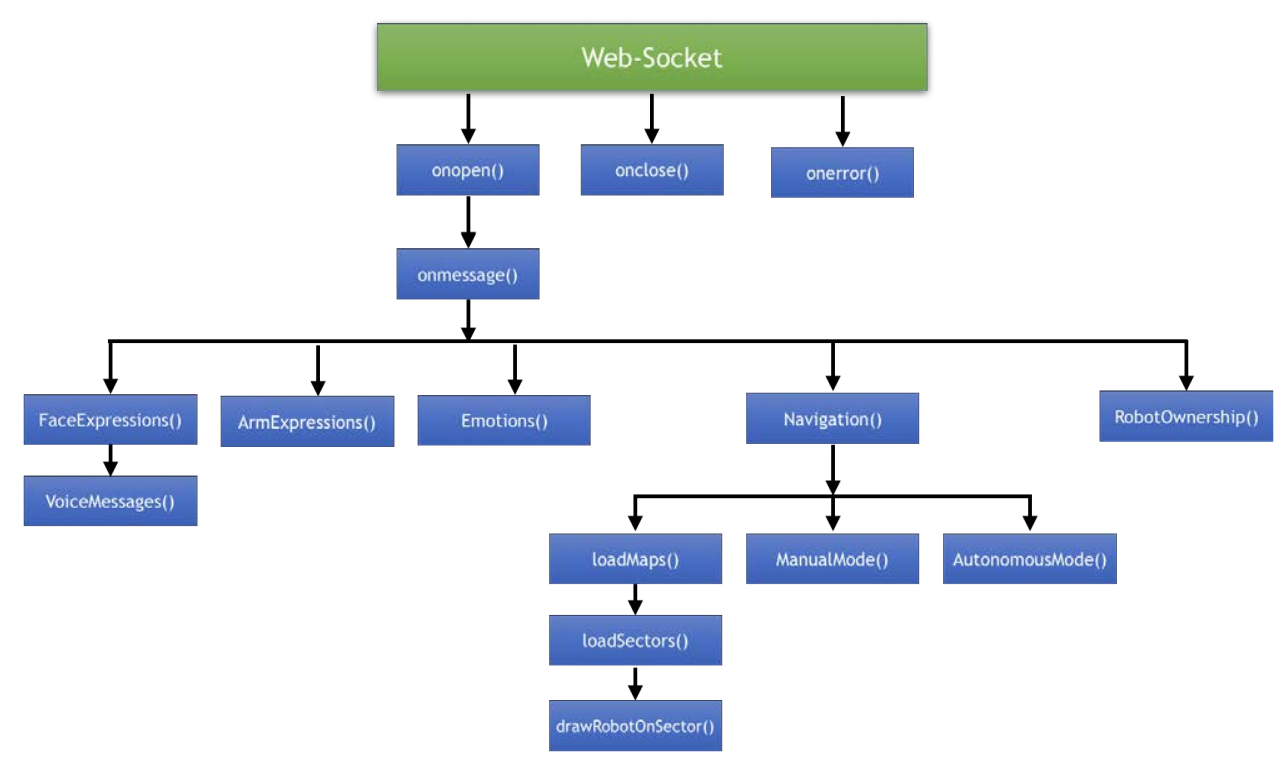

Figure 6.13: General connection scheme

The connection module is the most important component of this application as can be observed in the diagram shown in figure 6.13. While the connection with the robot exists, the information will be received and will be able to perform requests to the robots and to receive answers.

The rest of the modules depend on the reception of a message. Depending on the message this will activate one module or another from the diagram.

Two web-sockets are used in the application. The first socket is dedicated to normal request-answer messages, while another web-socket acts like a UDP socket. The use of this type of sockets are forbidden because they do not grant any security. Another websocket is called RTP (Real-time Transport Protocol) which acts like a UDP sockets but it is a normal TCP web-socket.

The web page is required to display in a single page. This type of applications is named SPA (Single Page Application) and their advantage is that they do not need to reload the whole web content when a change is produced, so the section affected is the only section reloaded the after the change is performed. For the SPA development AngularJS is used, which is an open framework developed in JavaScript.

\subsection{Experiments and results}

Once the specifications and the initial design are defined, the programming starts, and all definitions, classes and other components pre-established are converted into statements in Java, HTML and JavaScript. The next section shows some user interfaces of the 
applications developed.

In this dissertation it is very important to highlight that these types of applications do not "think", they only display the information provided by Doris and send back commands requested by the user.

\subsubsection{Android application}

In figure 6.14 the initial screen and the main menu can be appreciated. The menu is displayed by sliding the finger over the touch screen of the mobile device from left to right and the same menu is hidden when sliding the finger from right to left or pressing the back button of the device.
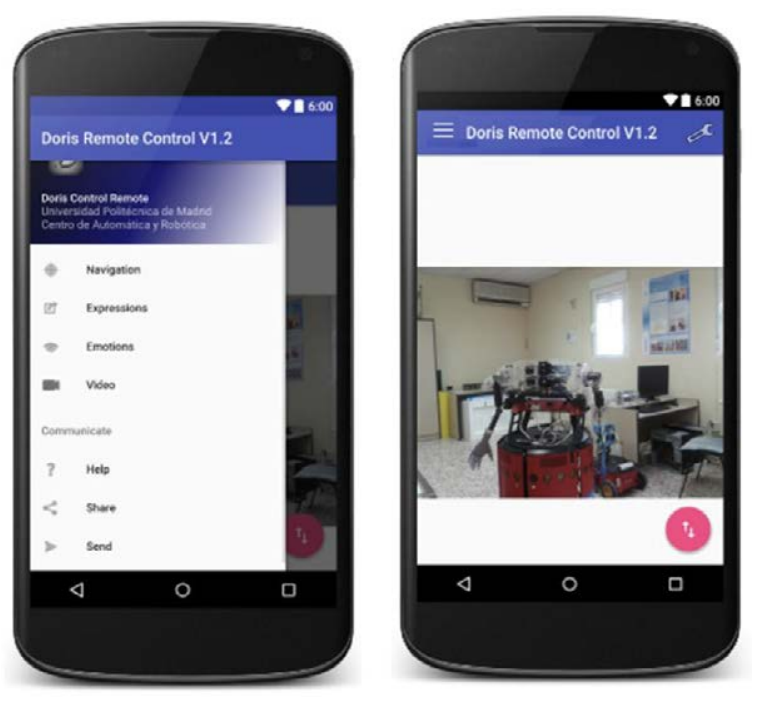

Figure 6.14: Initial screen of the android application

In the right-hand bottom corner there is a button with two arrows that shows an interface that enables the connection with other robots like Doris.

Figure 6.15 shows two notifications screen, the first one shows the arrival of a request, which informs the user that somebody wants to use the robot and the second one is the notification itself. These are very useful when the user is controlling the robot and notifies the user that the robot needs to be used. 


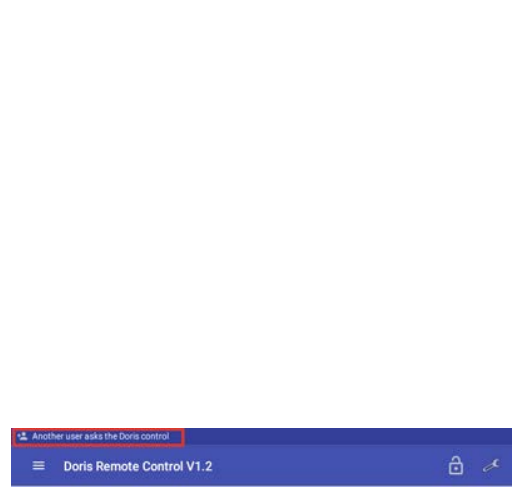

(a) Notification message

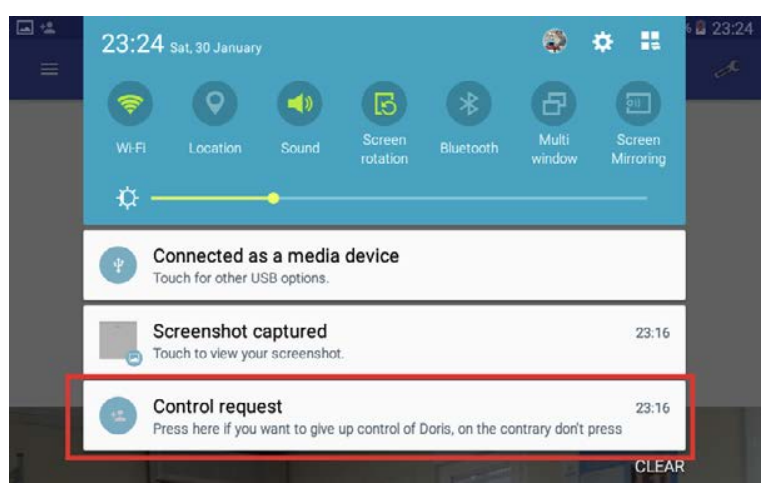

(b) Notification center

Figure 6.15: Notification of a permission request from another user

The expression screen shown in figure 6.16 is the interface to select manually a facial expression for Doris, which fulfils the basic requirement to display all the available faces in the server.

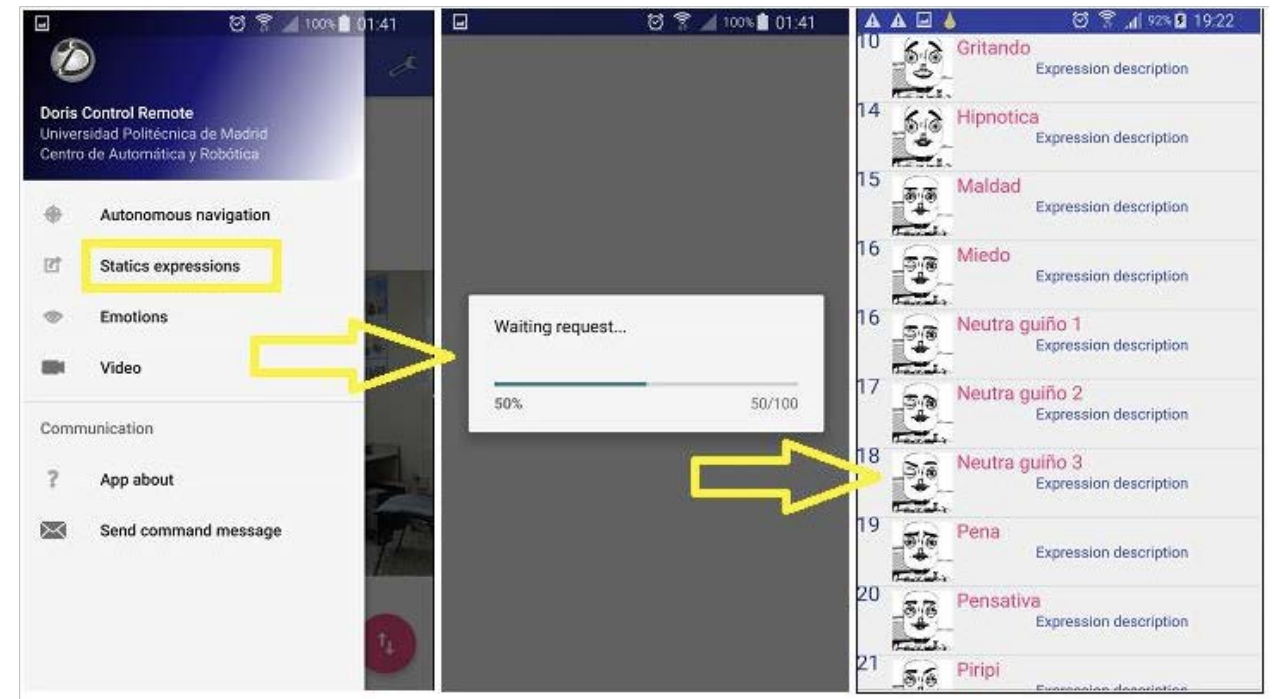

Figure 6.16: Android expressions screen

Figure 6.17 shows the manual navigation screen, fulfilling the requisite asked where two buttons act as a joystick providing Doris with the linear and angular velocities commands needed to move the platform forward and backwards and to spin it from left to right. 


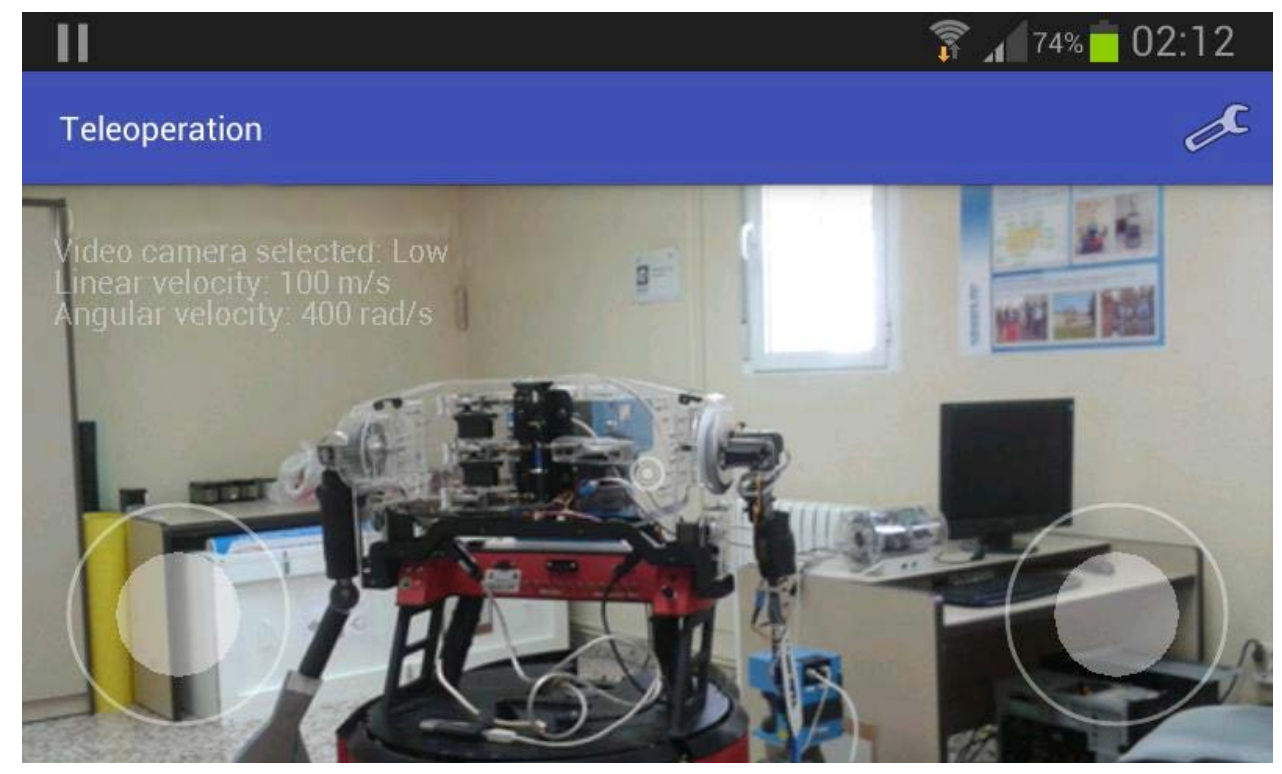

Figure 6.17: Android navigation screen

And the screen of settings is shown in the figure 6.18, an example of these settings consists in establishing the Doris' speed to move. It is remarkable that these settings are stored locally in the smartphone.

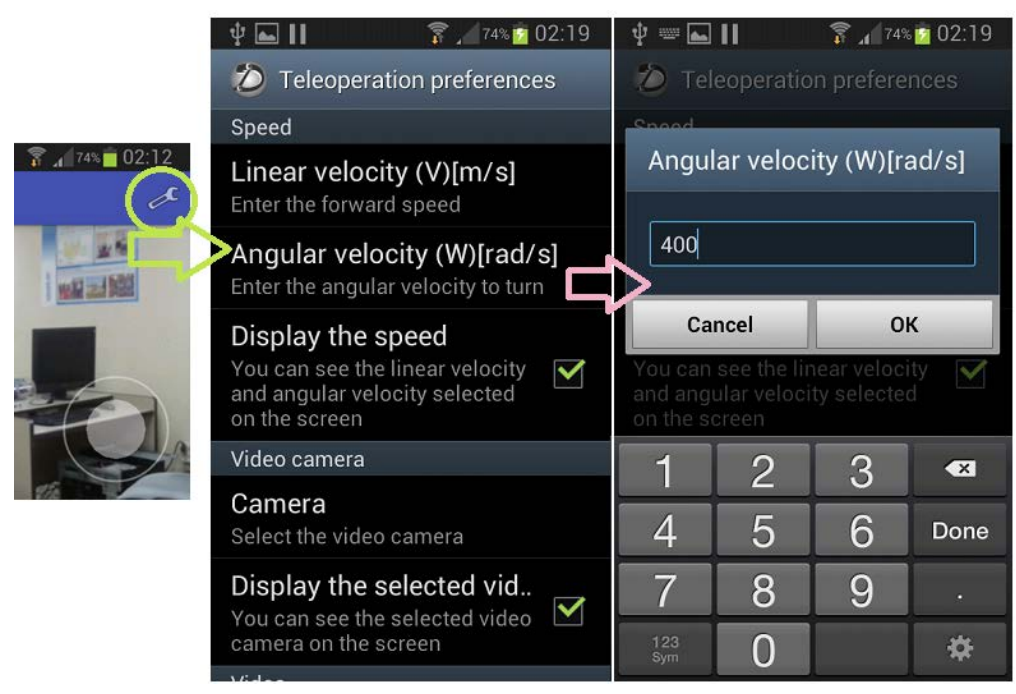

Figure 6.18: Current user settings screen

\subsubsection{Web application}

This application is installed in Doris as the web server with the support provided by Apache. This application has been developed in HTML 5, programmed in Javascript and JQuery and using the frameworks Bootstrap and Angular JS. The main web-page index.html, the libraries described before and the scripts developed for each section are 
loaded and the connection through the web socket is performed. If the connection is successful an icon that shows the connection status with Doris will turn green and it will keep in that state while the connection to the robot persists. If a disconnection occurs, the icon will turn red.

There is also another icon in a keypad shape that shows the token owner, if the keypad is green, it means that the robot is being controlled by the current user, if it is black it means that the robot is controlled by another user.

As the requirements specified for the android application, the figure 6.19 shows a new page that allows the user to modify the current Doris expression. The expressions are shown in the list placed at the left of the screen and the expression is sent to Doris when one item of the list is clicked.

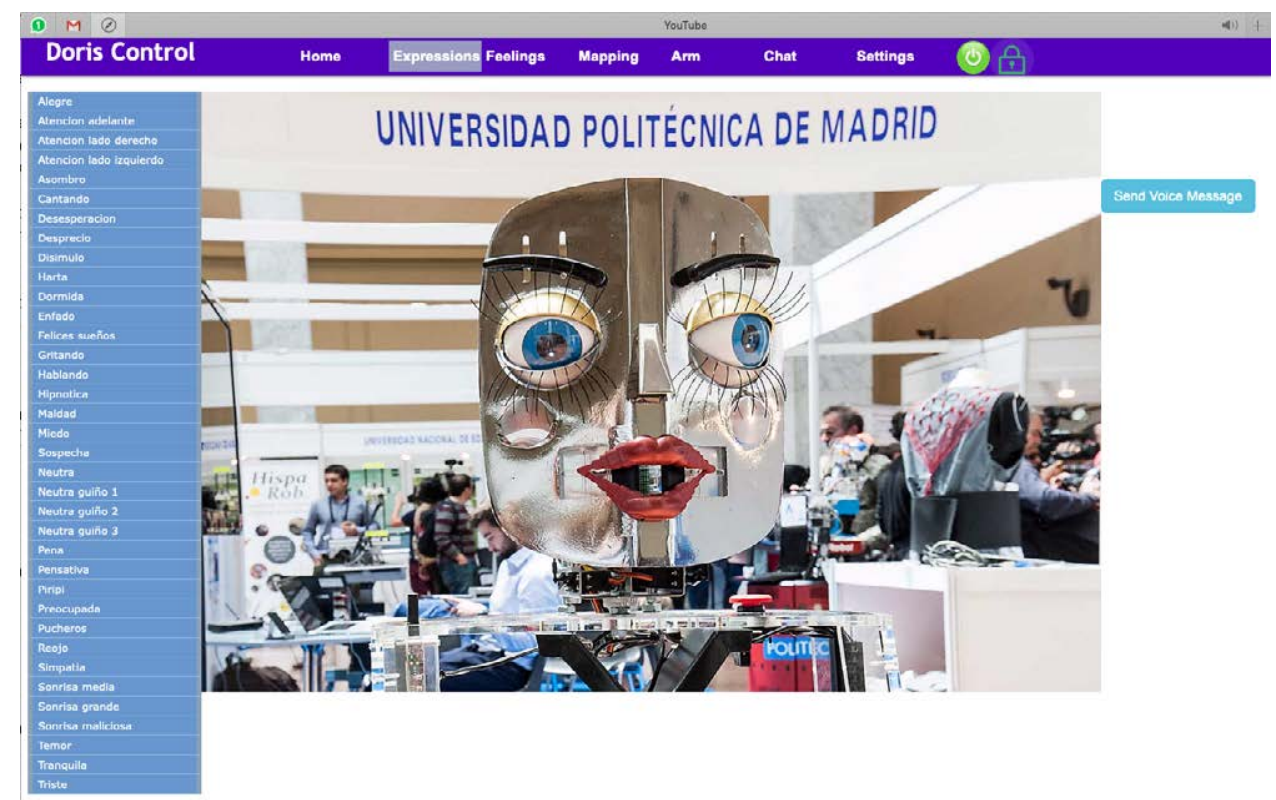

Figure 6.19: Expression Section

Figure 6.20 shows a set of charts where the evolution of Doris emotions are displayed. These emotions are received over the RTP socket connection, always having into account that the emotions will vary when a user speaks to Doris. The set of emotions displayed in the web page is explained in the chapter 5 taking into account that the range of values goes from $[0,100]$ 


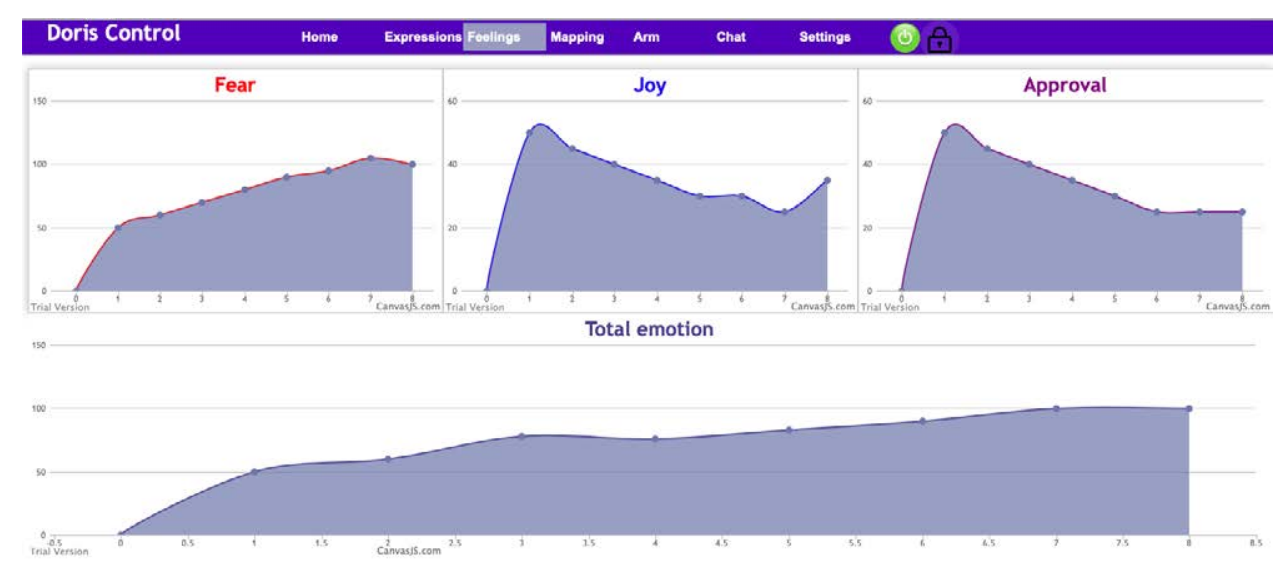

Figure 6.20: Emotions Section

Figure 6.21 allows the user to know or establish the current map where Doris is performing a tour. This enables the manual/autonomous navigation mode. The autonomous navigation mode is enabled when a program from the list is selected and then pressed to button Start Tour. When the arrows are pressed the autonomous mode is overriden and the robot starts to operate in manual mode.

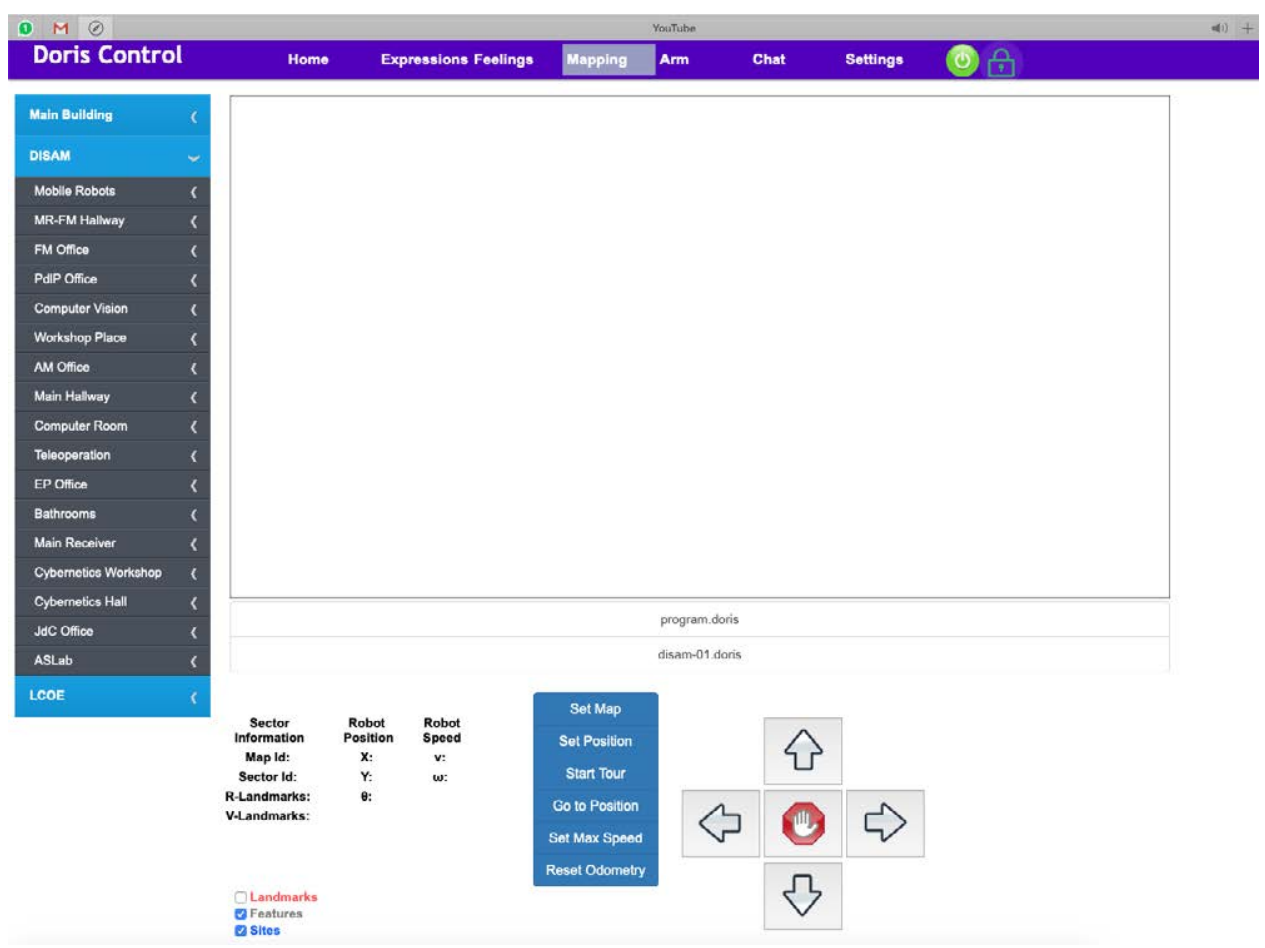

Figure 6.21: Mapping section

Other elements are present in this web page which are listed below:

- Arm: Future implementation of an interface to control a robotic arm to be installed 
on Doris. As the robot does not have installed the robotic arm, it has not been necessary to enable this tab.

- Chat: A chat window is displayed where different users of Doris can interact.

- Settings: Configuration of the different aspects of the application.

\subsection{Conclusions}

The field of robot teleoperation combined with the flexibility of web technologies like HTML5, AngularJS, provide a good solution for remote human-machine interaction.

For local control, an android application was developed following the process of software development. Some UML diagrams are shown in order to have a little knowledge of how the software should be designed and worked. Although this application is conceived to operate in a local network, it can be configured to operate in a remote place. The most important element is that the mobile device has internet connection.

One of the goals of this dissertation was to control Doris remotely. As Doris is based on a client-server application, it is able to handle many clients to connect simultaneously but only one can control it, being able to supervise and control the robot in the distance. The advantage of a web application is that it could be multi-platform, but the main disadvantage is the delay on communications when it is operated remotely.

Another goal of this dissertation was to develop a local application that can be installed in a mobile device like a mobile phone or a tablet, also working as a client application. 


\section{Task planning: a new interpreter}

Once all the modules of the proposed Doris' architecture were developed, they need to be integrated to work simultaneously. A task planner is proposed in this dissertation. This proposed task planner integrates navigation, face and emotions by means of a tasks list, which is a source code of a program language developed for Doris with which the robot's user can create or modify the order in which actions are going to be performed by the robot.

Some questions may be posed now: Why is it necessary to introduce a new programming language in a social robot if there are thousands of them already? And, if the robot is intended to work as a tour-guide, why are not its own actions directly programmed in its core program? The most adequate and reasonable answer given is that each robot's user could design a program in order for the robot to act as the user wants by simply using an easy programming language without the necessity of recompiling the core program which manipulates the robot, always remembering that Doris is intended to work in close and changing environments in which different events can take place.

Once it has been understood that a tour consists in a set of actions performed by Doris, the idea of creating a programming language appears. Hence, this programming language can be defined as any other simple programming language but that includes direct commands of Doris' movements and configuration regarding what, when and how sentences are going to be uttered as well as configuration regarding the performance of actions once the output has been perceived by the sensors data.

Therefore, this dissertation also includes information about the development of this language and its respective grammar, about how to read a source code program and about how to identify the list of reserved words in the language, which is known as lexical analysis. Once the tokens have been obtained, the structure of the statements is analyzed (syntax analysis). The semantical analysis is the last step to perform before the robot's execution of tasks. Finally, experiments and conclusions of the interpreter are performed. 


\subsection{Requirements of the language}

Grammar, which is going to be used to program the sequence of actions for Doris, must contain common features of all programming languages like input/output instructions, conditionals clauses, loops, arithmetic operations and boolean operations. Besides these features, the new programming language for Doris allows variables definition which can be either numeric or literals or defined as an array which can contain both numeric and literals in the same vector.

\subsection{General grammar structure}

To begin with the analysis of the source program, a grammar is needed in order to identify the tokens. Grammar 7.1 specify the grammar used for Doris Interpreter. Once known all the symbols, these will feed the lexical analyzer to build the tokens list.

where different types of statements and basic grammar are detailed as how to call a function or how literals and numbers are defined. A basic program is shown as next:

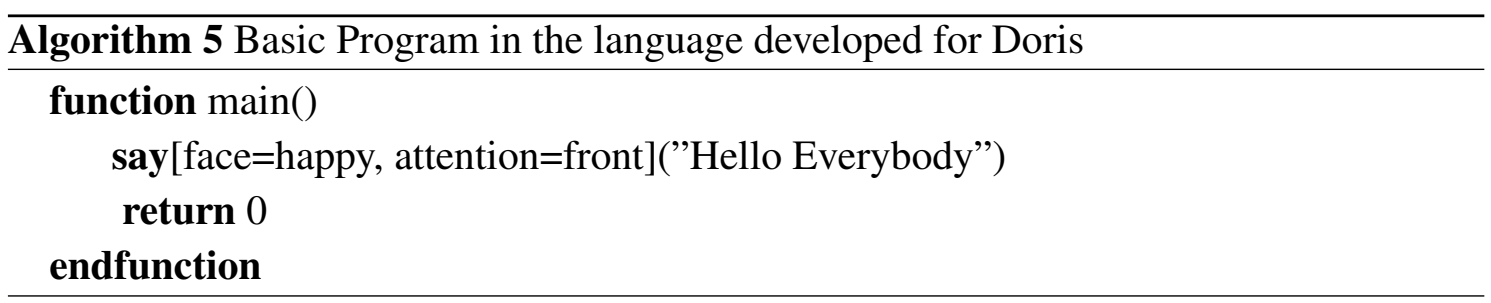

\subsubsection{The if/else statement}

The if statement is used for conditional execution.

$\langle$ if_stmt $\rangle::=$ 'if' '(' $\langle$ expression $\rangle$ ')' $\langle$ statement-list $\rangle$ ['else' $\langle$ statement-list $\rangle]$ 'endif'

The language developed for Doris should be able to allow the execution of a group of instructions when certain expression is true. In cases where that condition is false, a suite of the else clause is executed if present

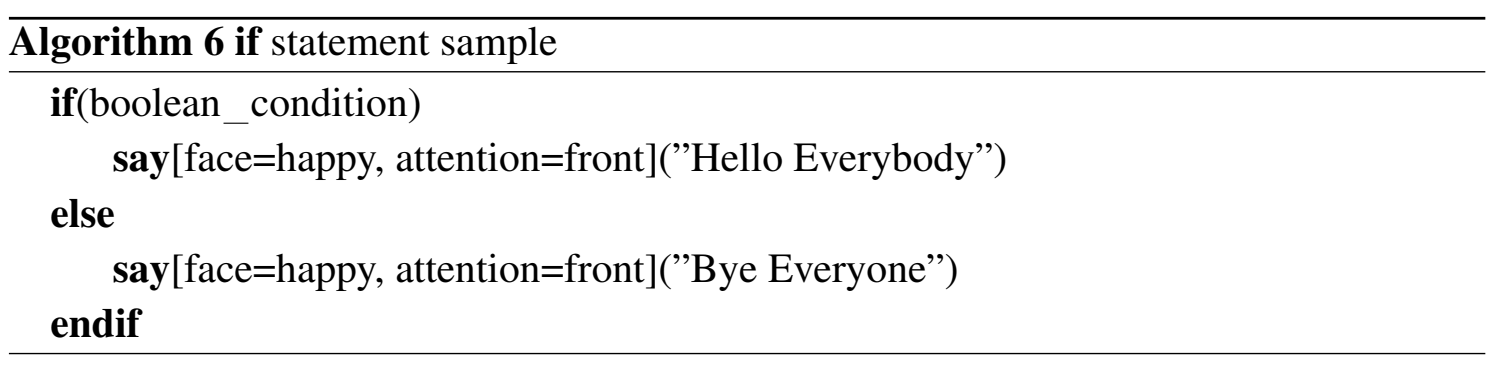




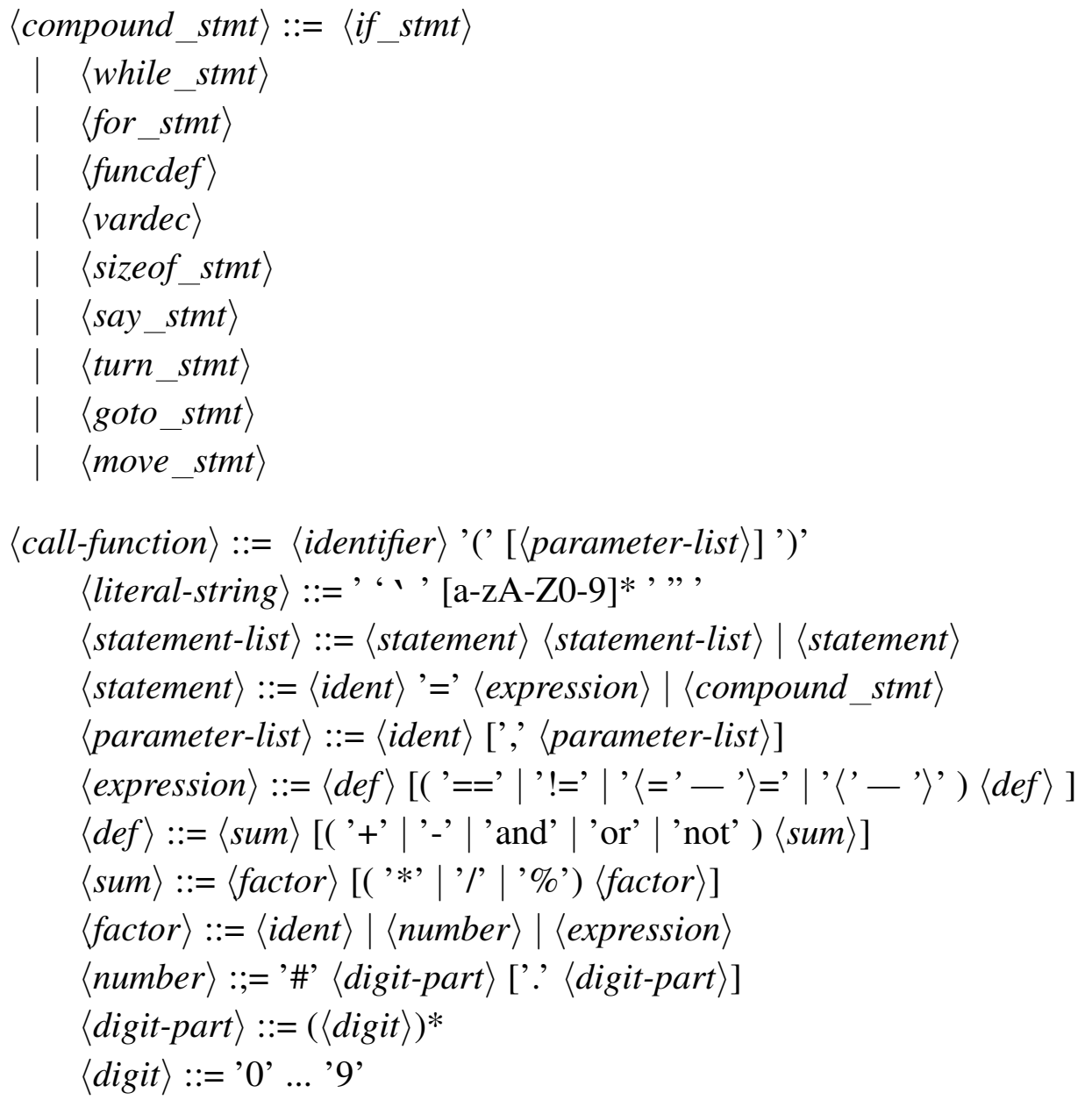

Grammar 7.1: Grammar specification for Doris interpreter 


\subsubsection{The while statement}

The while sentence is used for repeating the execution of a block of statements while certain condition is true.

$\langle$ while_stmt $\rangle::=$ 'while' '(' $\langle$ expression $\rangle$ ')' $\langle$ statement-list $\rangle$ 'endw'

This instruction is needed in cases where Doris is intended to repeat certain tasks, like asking the same question or moving forward until some point is reached.

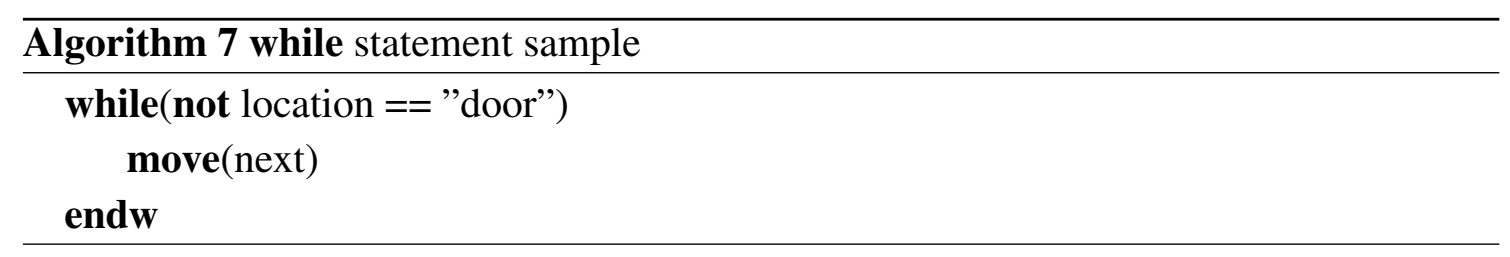

\subsubsection{The for statement}

The for is used to iterate over a sequence of elements like a list or an array, for instance, a list of tags read from RFID sensor.

$$
\langle\text { for_stmt }\rangle::=\text { 'for' '(' }\langle\text { expression }\rangle \text { ':' }\langle\text { expression }\rangle \text { ':' }\langle\text { expression }\rangle \text { ')' }\langle\text { statement-list }\rangle
$$

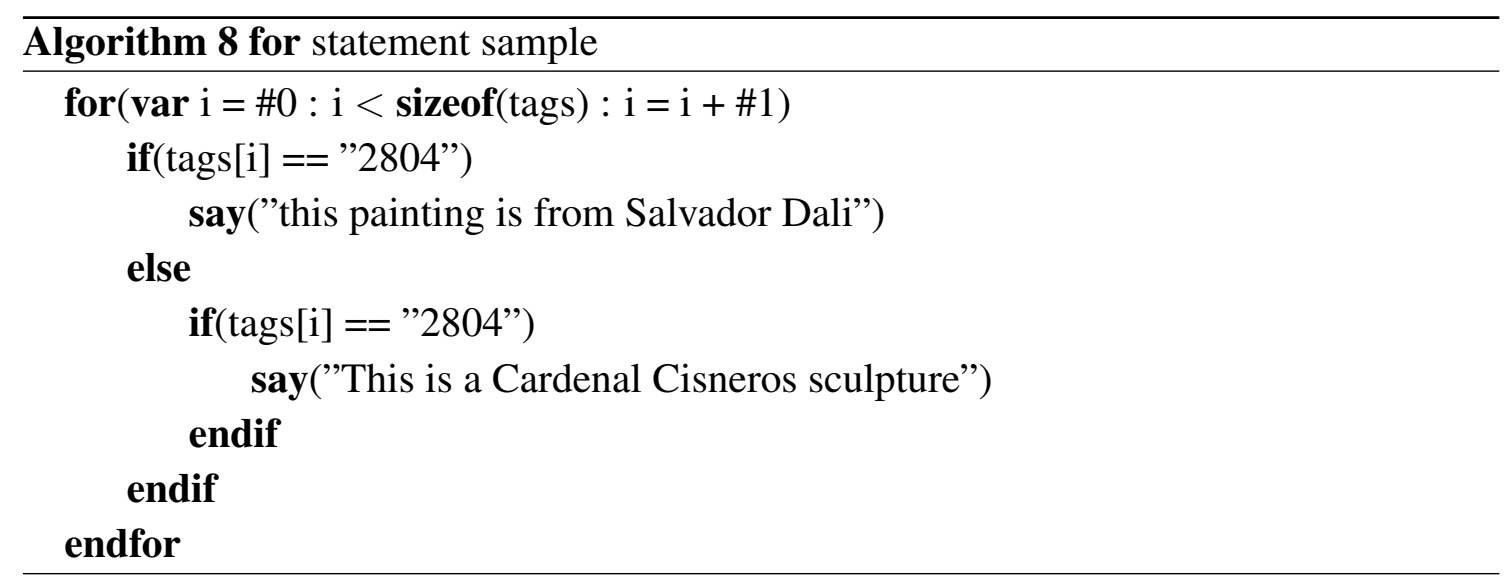

As seen in algorithm 8 , the statement is very similar to $\mathrm{C}$ language for statement, which firstly is a declaration of a variable, then, secondly, the condition and finally the upgrade of the variable. 


\subsubsection{Function definitions}

Functional programming has always been a very good idea in all programming languages. This was included in order to tell Doris the principal function to be executed and also create action routines which can be called or not from the main function or other functions.

The grammar is expressed as next:

$\langle$ funcdef $\rangle::=$ 'function' $\langle$ ident $\rangle \quad$ '([ $\langle$ parameter-list $\rangle]$ ')' $\langle$ statement-list $\rangle \quad$ ['return' $\langle$ expression $\rangle$ ] 'endfunction'

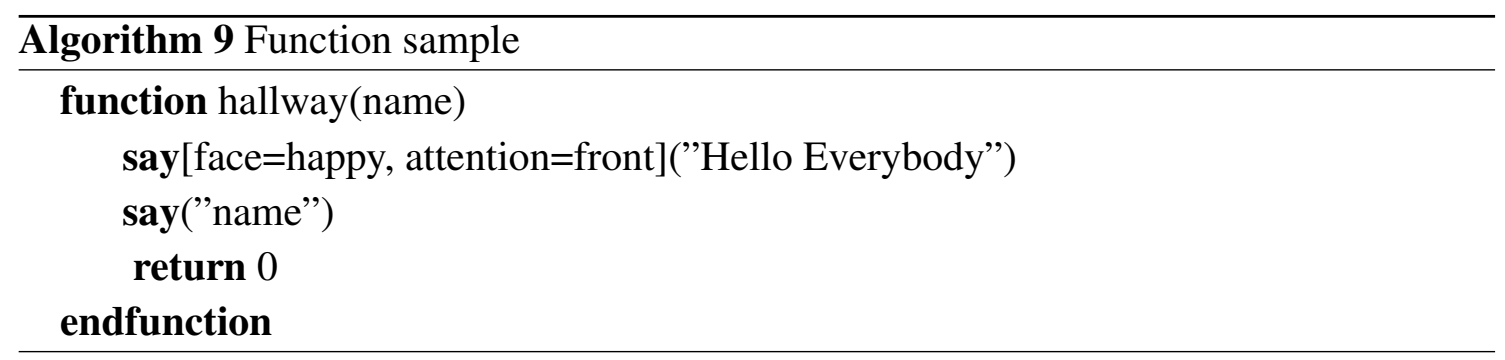

A new set of statements can be declared into the function, and it can contain parameters passed by values and also return a value if the return clause is stated.

\subsubsection{Variable declaration}

As in every language, variables are used for storing information. For Doris they are very helpful to handle information coming from sensors, specially the information provided from the sensors. The grammar is:
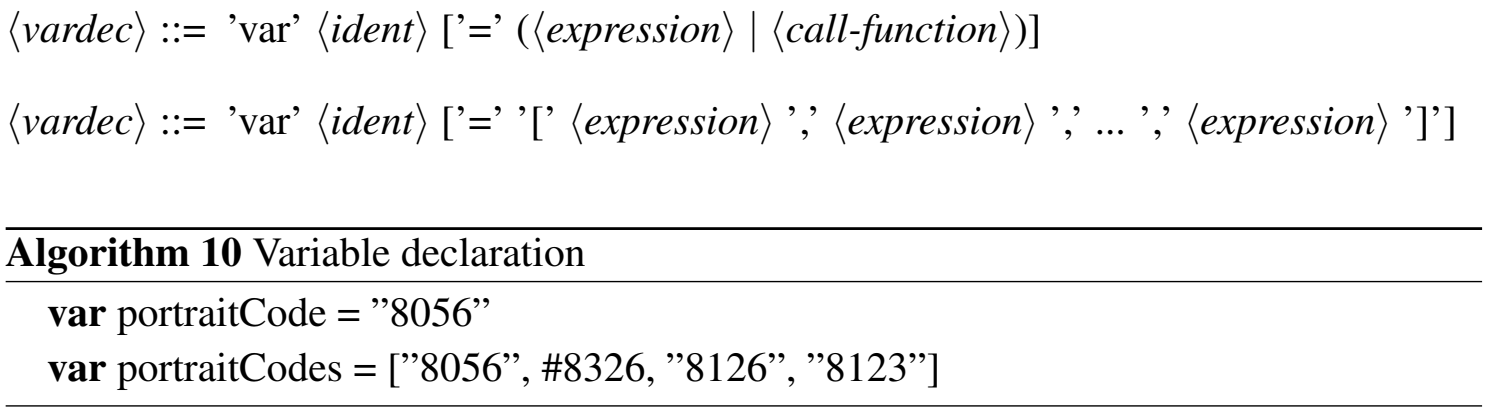

\subsubsection{The say statement}

This command indicates that Doris needs to say a specific phrase. 
$\langle$ say_stmt $\rangle::=$ 'say' [ '[' 'attention=' $\langle$ attention-item $\rangle$ ';' 'face=' $\langle$ face-item $\rangle$ ']' ] '(' $\langle$ literal-string '’',

As it can be observed in the grammar, the instruction can be executed with extra options. These options are concerning to the state of the face and the attention direction.

\begin{tabular}{l}
\hline Algorithm 11 say instruction \\
\hline say("My name is Doris") \\
say[face=smiley; attention=left]("Let's move to the left side of the room") \\
\hline
\end{tabular}

\subsubsection{The turn statement}

The turn command makes the robot turn the amount of degrees specified as a parameter. The degrees can be specified as a variable, as a result of a function or writing the value in degrees.

$\langle$ turn_stmt $\rangle::=$ 'turn' '(' $(\langle$ number $\rangle \mid\langle$ ident $\rangle \mid\langle$ expression $\left.\rangle))^{\prime}\right)$ '

\begin{tabular}{l}
$\overline{\text { Algorithm } 12 \text { turn instruction }}$ \\
\hline turn( $\# 90)$
\end{tabular}

\subsubsection{The move statement}

The move command tells the robot to move to the next or the previous waypoint specified in the tour database.

$\langle$ move_stmt $\rangle::=$ 'move' '(' ('next' | 'prev') ')'

\begin{tabular}{l}
\hline Algorithm 13 move instruction \\
\hline move(next)
\end{tabular}

\subsubsection{The goto statement}

The goto command indicates the robot where to go. The grammar is specified here under:
$\langle$ goto_stmt $\rangle::=$ 'goto' '(' (〈number $\rangle \mid\langle$ ident $\rangle \mid\langle$ expression $\rangle)$ ', $(\langle$ number $\rangle \mid\langle$ ident $\rangle \mid$ $\langle$ expression $\left.\rangle)^{\prime}, '(\langle\text { number }\rangle \mid\langle\text { ident }\rangle \mid\langle\text { expression }\rangle)^{\prime}\right){ }^{\prime}$


The function contains three values: the first one is the sector identifier in the same map, if \#-1 is especified, the Robot will remain in the same sector where it is located, and the other two parameters are position $\mathrm{X}$ and $\mathrm{Y}$ in the plane and inside the sector boundaries. For instance:

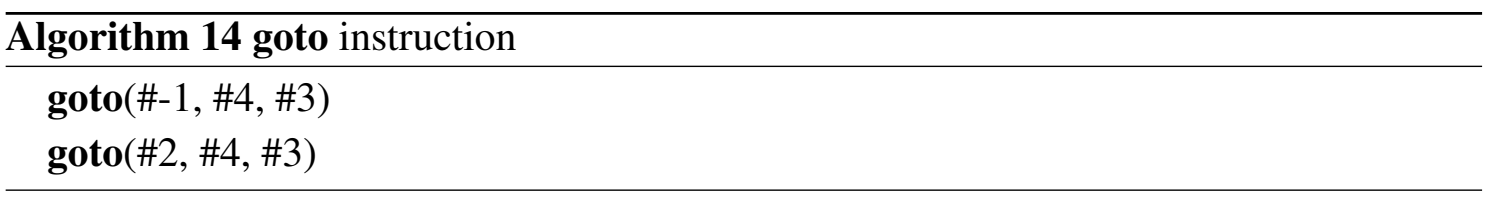

\subsubsection{The sizeof statement}

The sizeof command returns the current size of an array. If the variable specified is not an array, it will return 1;

$\langle$ sizeof_stmt $\rangle::=$ 'sizeof' '(' $\langle$ ident $\rangle$ ')'

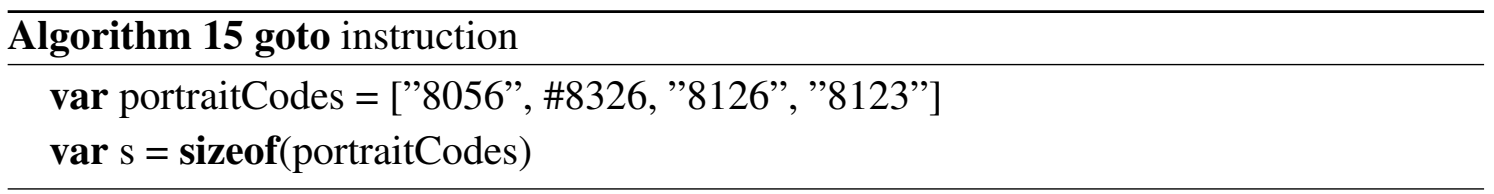

\subsection{The lex() function}

As previously explained, the lexical analysis is commissioned to recognize the expressions according to the specified grammar and store them as a token list, to be used later by the syntax analyzer. For this dissertation, a lex function is developed to perform this initial task. lex() reads the source code file and process each character, resulting in the required token list. The next section will show the tokens to be detected and how they are handled and inserted in the token list.

$<\mathbf{I F}>$ : if the lexical analyzer finds the if statement this will create the next token list: [IF, EXP: <expression>, BIF:0, <statement-list>, BEL:0, <statement-list>,EIF:0]. If the statement is well formed, after the IF token will follow an expression denoted with the EXP token, where the whole expression is copied into the token list to be tokenized in another moment. Then, three more tokens are added, which are:

- BIF:id. This token identifies the beginning of the if statement. The id identifies the if-else statements correspondence.

- BEL:id: This token defines the beginning of the else statement. If the if statement does not contain an else statement, the statement-list will be empty. 
- EIF:id. The token denotes the end of the if statement.

$<$ WHILE $>$ : during the lexical analyzer, it can be found the while statement. This will create the next token list: [WHILE, EXP: <expression $>$, BWH:0, <statementlist $>, E W H: 0]$. It contains similar extra tokens like the if token. These are:

- BWH:id. This token identifies the beginning of the while statement. The id identifies the while statement and match its beginning to its end.

- EWH:id. The token denotes the end of the while statement.

$<$ FOR $>$ : the token list will be as follows: [FOR,EXP: <expression $>$,EXP: $<$ expression $>$, EXP: $<$ expression $>$, BFR:0, <statement-list $>$,EFR:0].

- BFR:id. This token identifies the beginning of the for statement. The id identifies the for statement and matches its beginning to its end.

- EFR:id. The token denotes the end of the for statement.

$<$ DVR $>$ : This token identifies a variable declaration. The token list will be as follows: [DVR: $<\mathrm{id}>$ ]. As the variable declaration can be initialiazed with a value, with another variable or from a function, the token list will be as follows: [DVR: $<$ id $>, E Q$, EXP: <expression $>$ ].

$<\mathbf{S A Y}>$ : The token indicates that Doris must say something to the audience. The token is followed by a string token that indicates the phrase which Doris must say. The token list will be as follows: [SAY, STR: <string $>$ ]. The say instruction can contain optional arguments like the face expression and the head position. These options are stored in the token list will be as: [SAY, OPT:<options $>$, STR: $<$ string $>$ ].

$<$ TURN $>$ : turn statement is stored inside the token as: [TURN, NUM: $<$ number $>$ ].

$<$ MOVE $>$ : This token is stored as [MOVE, VAR: $<\mathrm{id}>$ ].

$<$ GOTO $>$ : This token is stored as [GOTO, EXP: <expression $>$, EXP: $<$ expression $>$, EXP: $<$ expression $>$ ].

$<$ SIZEOF $>$ : This token is stored as [SIZEOF, EXP: $<$ expression $>$ ].

There is an another token which is created when a function returns a value, and this value needs to be stored in a variable. So the function is treated as an expression referencing the token list as: $[F C N,<$ parameters-list $>$ EFC]. Finally, there are other tokens like: 
- $<\mathbf{V A R}>$, which defines that a variable is being used.

- $<$ NUM:value $>$, meaning that a number is stored in the token list. All numbers in the interpreter are treated as float.

- $<$ STR:value $>$, meaning that a character string is stored in the token list.

- $<$ EXP:value $>$, an expression is stored to be analyzed by another lexer during the parsing.

- $<$ ARR:value $>$, Defining an array of elements.

\subsection{The parse() function}

Once the lexical analysis of an input text is completed, the next step in the process of creating an interpreted language is to convert the token list into actions. This involves both, syntax and semantical analysis, in the same function. It is important to remark that nothing will be performed without a main function. This is the start point of execution of the interpreter, very similar to what languages such as Java or $\mathrm{C} / \mathrm{C}++$ do.

Combination of tokens will activate the different submodules of the architecture proposed which will turn into the set of actions that Doris is intended to perform during the tour in the museum. It is important to remember that the $<$ EXP:value $>$ token needs to be processed by another lexer analyzer. This will result in another tokens list that will be used by a function called expressionSolver.

The expressionSolver is based on a recursive descent parser. This is a LL(k) parser using one token of lookahead $(\mathrm{k}=1)$ with backtracking, and it is used to solve conditions, factors and terms. The result of the descent parser will be one of two options:

- Boolean: a token with numerical value zero $(<\mathbf{N U M : 0 ~}>)$ indicating that the condition is false or with a numerical value one $(<\mathbf{N U M : 1}>)$ indicating that the expression is true.

- Numerical: another token with any numerical value.

\subsection{Experiments and results}

To test the interpreted language, a simple program is created to send Doris from the current sector to another sector. Input file is shown in listing 7.1 
Listing 7.1: Program performing a simple plannification

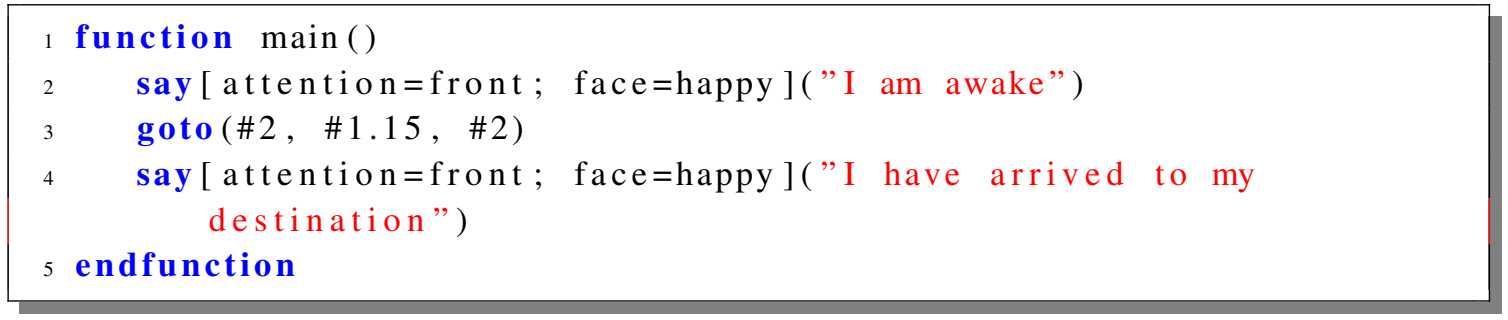

Three lines are executed in the main function of the code listed below, but with this action each instruction generates a set of actions like, setting a facial expression, and saying a text stated by the programmer, involving the Text to Speech module and the lips syncing module.

Another instruction makes the robot move to another place. This involves, the navigation, control and planification system. This involves using the sensors installed and proposed in the architecture. This makes all the submodules join into one single execution. Figures shown in 7.1, 7.2 and 7.3 display transitions of Doris while performing the program listed below.

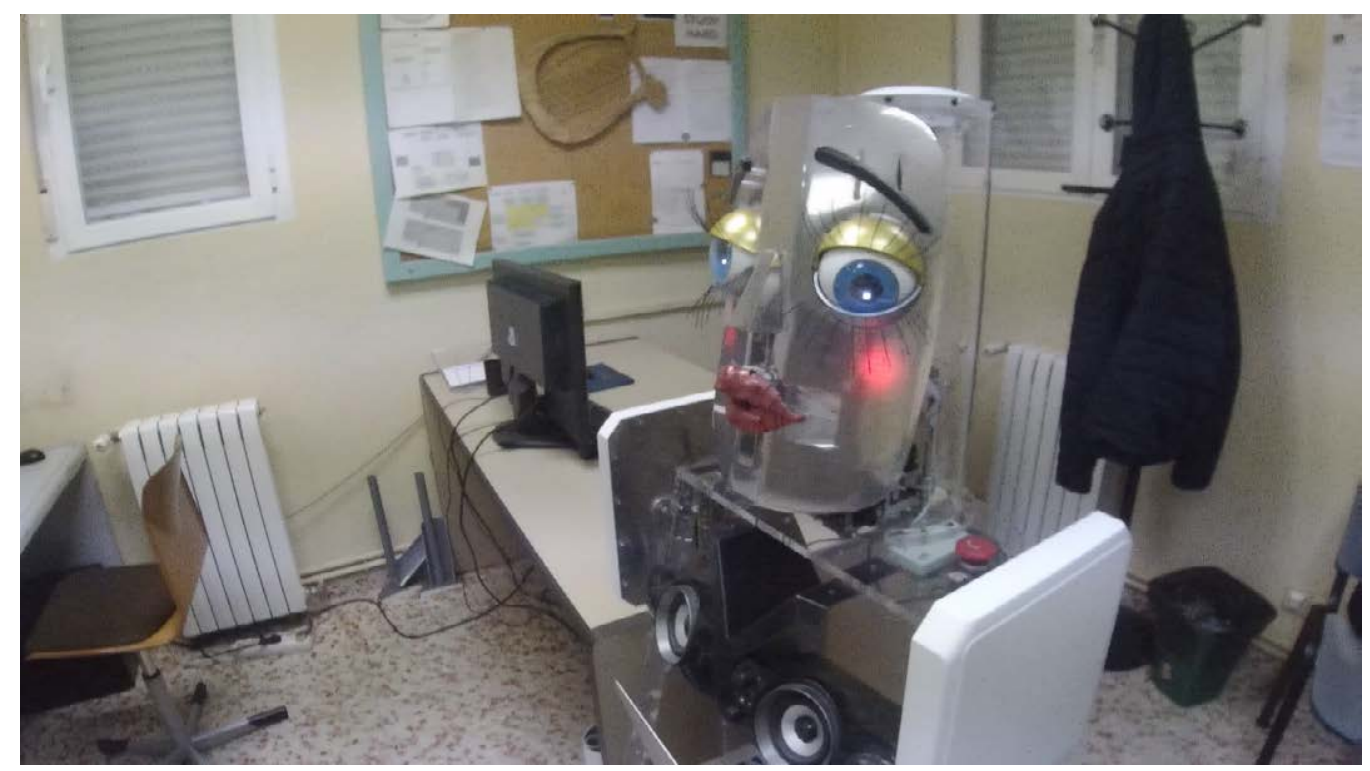

Figure 7.1: Doris awakening to perform her first tour 


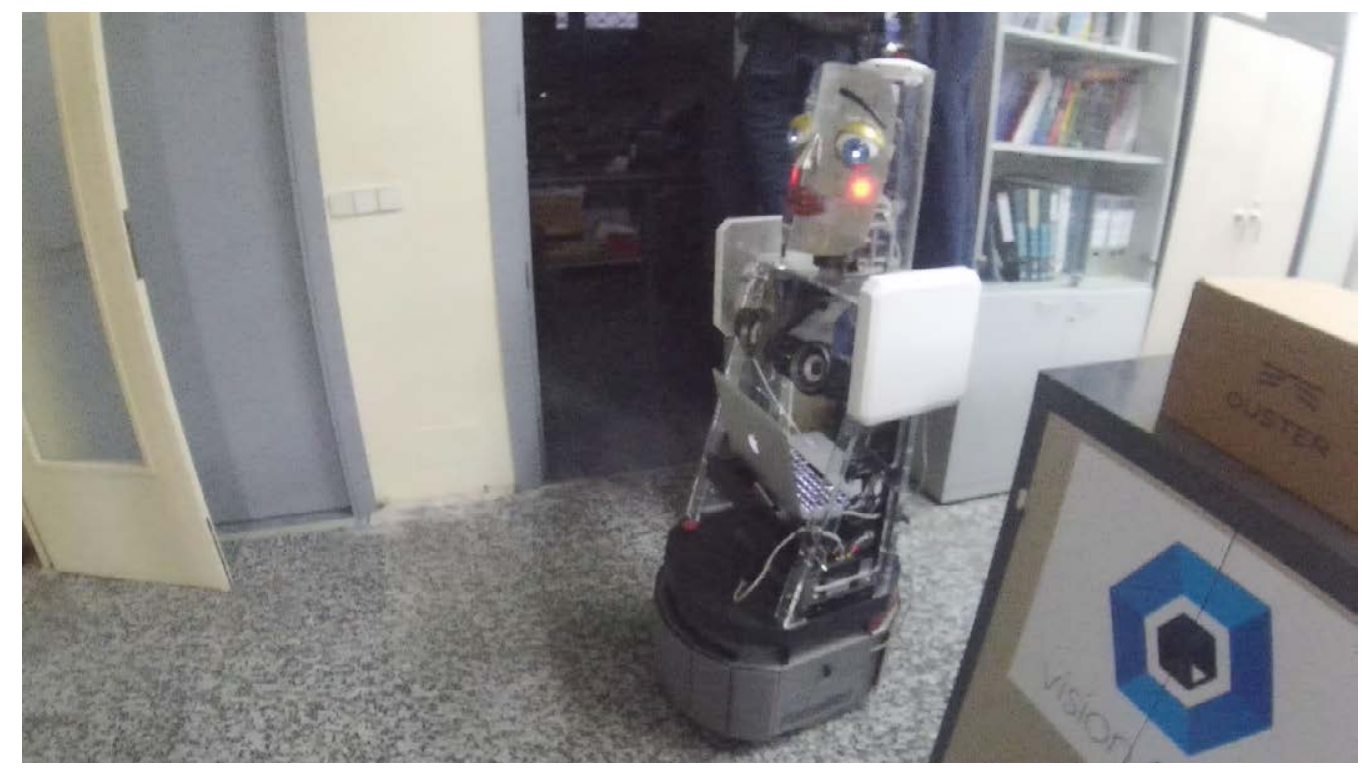

Figure 7.2: Doris moving to a point on her first tour

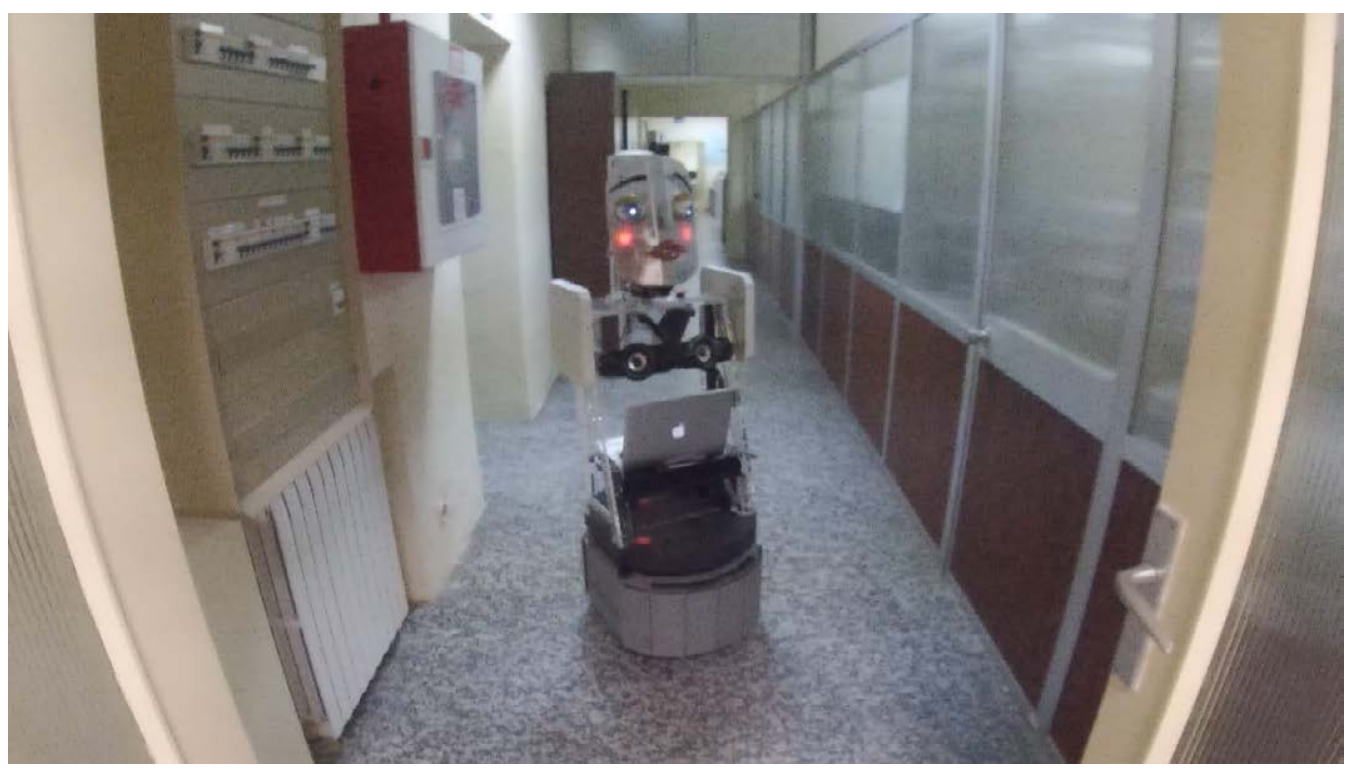

Figure 7.3: Doris arrival to the desired point

Another program that involves touring in the Centre of Automation and Robotics at Universidad Politécnica de Madrid has been developed. The program is listed in listing 7.2. The program consists in going to the entrance of the laboratory and speaking to the visitors. Then the program makes the robot move to the first location which is going to be explained. As stated, explanations are based on points of interest which were previously defined, thus, when the robot arrives to the point it executes the following command. In this specific case, the commands that provide an explanation are the say instruction.

As it can be observed, events are included in the program. These events are: 
1. onRfidTagsDetected: which subscribes a function to the event and allows the interpreter to know what to do with the tags.

2. onSectorChange: which notifies to the interpreter what to do when a new sector is loaded in the robot.

Listing 7.2: Program that performs a tour in the Centre of Automation and Robotics

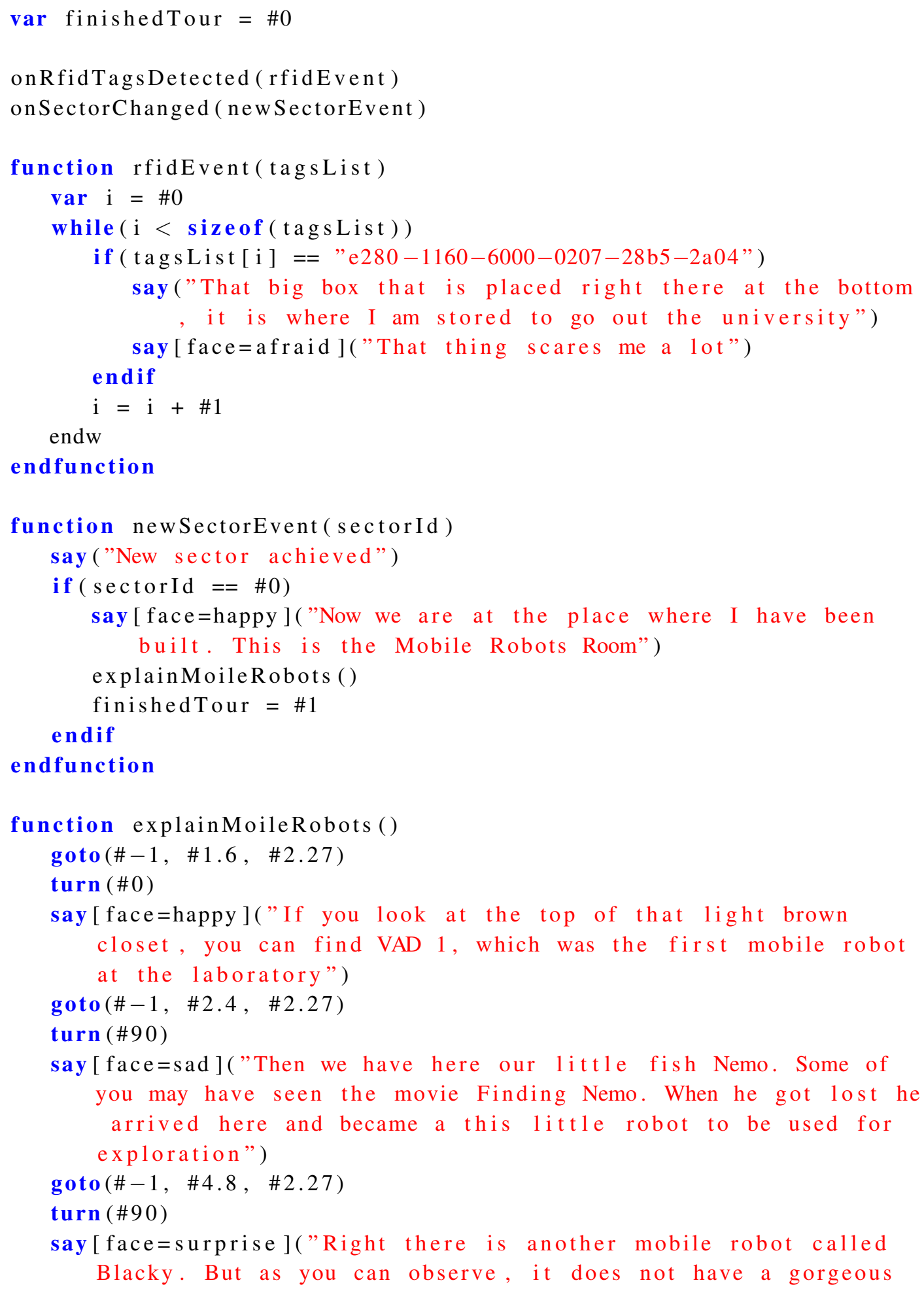




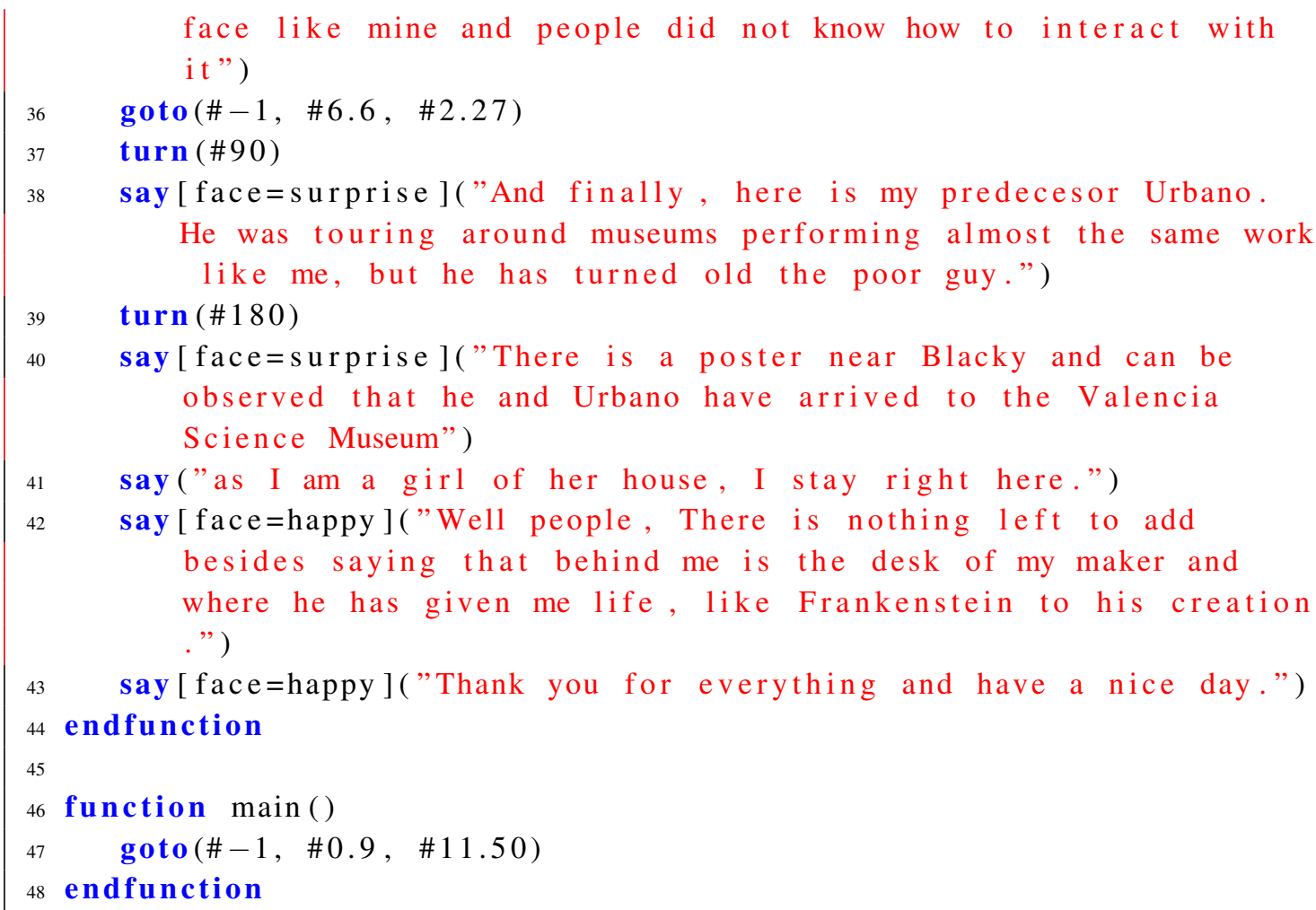

A full video, named Full System Test: Touring in the Laboratory, where the robot is performing the program mentioned above can be found at the group's webpage gallery Intelligent Control Group UPM-CSIC - Gallery

\subsection{Conclusions}

A new programming language is proposed for task planning so that an interactive robot can work in laboratories or museums. This programming language integrates different components of the robot like the sensors, face and voice interaction, emotions, navigation, etc. The new language is a mixutre between $\mathrm{C}$, Pascal and JavaScript languages which can be written in a more simpler way than other languages developed at the moment for robot's task planning.

The programming language implements different elements such as basic concepts of any programming language which are simple statements, conditional statements (IF), loop statements (WHILE, FOR), arithmetical and logical operations, and some other functions to manipulate the robot like moving it towards a point (GOTO), turning it to some orientation (TURN), saying something using an specific expression (SAY) and so on.

The use of events is the most important issue proposed and developed in this programming language, because they connect to different systems (the RFID or the 
speaking system). The language also integrates face and voice modules which need to be synchronized with lip movements and the navigation when arriving to a specific place. 


\section{Conclusions and future work}

Social robots, such as personal assistants, domestic helpers, etc are widely used nowadays. Thus, research in this area has increased. Interaction with robots, which is very hard to achieve, can be described as an important branch on robotics systems. When it comes to interaction, the main issue is that different people experience different reactions when interacting with robots.

NAO, MINERVA, URBANO, ROBOVIE and SOPHIA, among others, are interactive robots that have been designed to achieve different purposes. There are toy robots such as NAO, and interactive robots such as SOPHIA, whose physical appearance is so similar to humans that sometimes creates rejection among people around.

Another instance of interactive robots are tour-guides in museums. Hence, navigation and localization become important areas when developing robots that must move along indoor areas.

In the end, all these robots have common characteristics that are on permanent

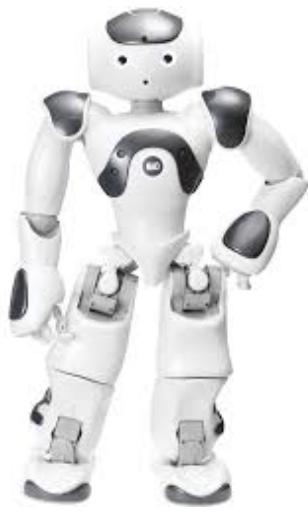

(a) NAO

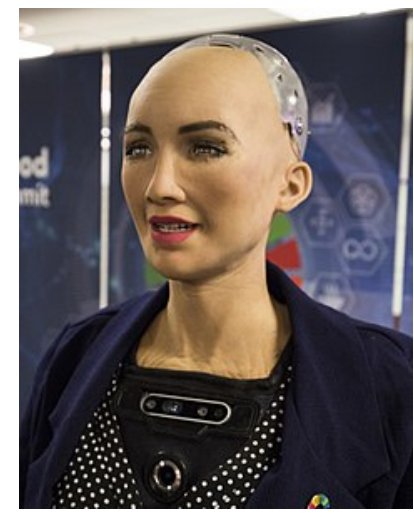

(b) Sophia

Figure 8.1: Robots for interaction 
research to improve or to create new methodologies that allow them a better performance when developing their respective tasks.

\subsection{Goals reached}

Several works concerning different architectures were mentioned in chapter 2, but most of them were focused on a single point of view such as localization, navigation or interaction, but not on all of them at the same time.

An architecture proposed in chapter 3 takes into account some parts from all the works mentioned previously but improving them and adding some new ideas. The proposals of this architecture are made in hardware and software.

The robot appearance must take into account the avoidance of the uncanny valley. One of the ideas to avoid this issue consists in adding emotions to the robot when interacting with it. And concerning the software, architecture proposes a multithreaded client-server system, which avoids the use of other types of systems like $\boldsymbol{R O S}$.

Indoor position estimation was developed in chapter 4 is extremely useful for guiding Doris in the museum. To help with localization, navigation and control, external sensors such as omnidirectional camera, RFID system and the laser range finder were added in order to achieve a better performance during the tour guide. The use of a laser range finder to detect reflective landmarks was a good idea, but building these type of beacons is a complicated process requiring expensive materials. The process of finding the best place to locate the reflective landmark was important, so that the classification algorithm could detect different landmarks, despite it being a good method to achieve localization without mapping the whole environment.

The omnidirectional camera used for localization is a very powerful sensor because it provides a 360 degree field of view of the built environment and it achieves reliable angle estimations. One big disadvantage of working with an omnidirectional camera is that a high resolution image is needed for detection that requires a fast processing speed. At present, Doris is not equipped with sufficient processing capacity and the image processing has to be performed by another computer.

The RFID system cannot be used for localization due to the high uncertainty that it presents. This is essentially caused by the low operating frequency of RFID. Ultra Wide Band systems work at very high frequencies that increase the efficiency of the performance. However, RFID was used for the navigation system by providing semantics to the environment which is stated into the semantic map.

Experiments demonstrate that even with obstacles or people passing by, or even in 
the absence of one type of the landmarks, a good localization, control, navigation and planning is achieved. It can be achieved by using only the omnidirectional camera, but can be refined by including the reflective landmarks.

The results are that Doris can locate on the presence of people and different objects located when both sensors are working at the same time. Although Doris can locate itself by only using the camera, there will be situations where both types of landmarks will not be easy to place or the museum does not allow to place the beacons or maybe it is too dark to place the visual markers, for this reason a sensor fusion was implemented. This methodology was developed in order to avoid using SLAM technique.

The integration considers the use of the navigation previously developed using the EKF with sensor fusion, but when interacting with people navigation needs to be adjusted in order to have a better performance, so the fuzzy sets of the speed and hallway controllers were modified to avoid conflicts when the robot was surrounded by people, and the integration of the RFID system helps with the sector identification using the semantic map. Thus, localization and navigation are optimized. The sector identification tags were placed in doors at $1.0 \mathrm{~m}$ of height and with the RFID system the power and the sensitivity of the antennas were calibrated in order to detect the tags only when the robot is passing through the door.

An interaction system was developed for Doris and it is detailed in chapter 5. Making a robot that can interact with different types of audiences is not easy. Despite this, the goal, which consisted in Doris' interaction with environment and the avoidance of the uncanny valley, was achieved. Doris' physical appearance is very well accepted among people.

The emotion system was developed in order to achieve a more realistic interaction. The system modifies the face and the voice and provides an output message to the visitor according to the emotion at that instant. A fuzzy engine was developed to achieve this goal.

The field of robot teleoperation was explored in chapter 6 developing android and web application to provide a good solution for remote human-machine interaction.

For local control, an android application was developed following the process of software development. For remote control a web application was developed. As Doris is based on a client-server application, it is able to handle many clients to connect simultaneously but only one can control it, being able to supervise and control the robot in the distance. The advantage of a web application is that it could be multi-platform, but the main disadvantage is the delay on communications when it is operated remotely.

A new programming language proposed for task planning, so that an interactive robot can work in laboratories or museums, was developed in chapter 7. This programming language integrates different components of the robot like the sensors, face and voice interaction, emotions, navigation, etc. The new language is a mixutre between $\mathrm{C}$, Pascal 
and JavaScript languages which can be written in a more simpler way than other languages developed at the moment for robot's task planning.

The programming language implements different elements such as basic concepts of any programming language which are simple statements, conditional statements (IF), loop statements (WHILE, FOR), arithmetical and logical operations, and some other functions to manipulate the robot like moving it towards a point (GOTO), turning it to some orientation (TURN), saying something using an specific expression (SAY) and so on.

The use of events is the most important issue proposed and developed in this programming language, because they connect to different systems (the RFID or the speaking system). The language also integrates face and voice modules which need to be synchronized with lip movements and the navigation when arriving to a specific place.

\subsection{Future developments}

Some topics must be considered for future developments that require a deeper and dedicated study. Some improvements can be made in hardware and software level which are listed next.

A complete control architecture was explained in chapter 3 detailing what hardware to use. Integrating an Ultrawide Band system can be one of these improvements on hardware level. Besides, a development of a new skeleton to enable the capacity to spin without moving the platform and to finally attach the robotic arm may be interesting. Regarding software, communication between threads can be optimized.

A sensor fusion with an EKF and using laser and omnidirectional camera was proposed in chapter 4. Changing landmarks or replacing them by using elements present in the environment like doors, windows and other stuffs very hard to move, can be an excellent improvement. Integrating the UWB system into the localization is also interesting. And finally, a sensor fusion technique with the Unscented Kalman Filter or a particle filter may be a good development.

An interaction system including the face, arm, voice and emotion system based on input text, was developed in chapter 5. A complete robotic arm redesign and add it to the emotion system represents a big improvement for interaction. Adding more variables to the emotion system is a good idea to explore. And finally, previous experiences that might affect Doris emotions is an interesting area to develop.

Clients applications were developed in chapter 6. Applications in general must be improved. An application for apple devices might be developed. android application 
needs to be potentiated and in the web application a module to develop the programs for Doris needs to be incuded.

The interpreter, which acts as a task planner was developed in chapter77. Concurrence and instructions that can interact with the hardware can be included. 


\section{Bibliography}

[1] S. Thrun, M. Beetz, M. Bennewitz, W. Burgard, A. Cremers, F. Dellaert, D. Fox, D. Hahnel, C. Rosenberg, N. Roy, J. Schulte, and D. Schulz, "Probabilistic algorithms and the interactive museum tour-guide minerva," Journal of Robotics Research, vol. 19, pp. 972-999, November 2000.

[2] W. Burgard, A. B. Cremers, D. Fox, D. Hähnel, G. Lakemeyer, D. Schulz, W. Steiner, and S. Thrun, "Experiences with an interactive museum tour-guide robot," Elsevier Artificial Intelligence, vol. 114, pp. 3-55, October 1999.

[3] R. G. Boboc, M. Horaiu, and D. Talabă, "An educational humanoid laboratory tour guide robot," Procedia - Social and Behavioral Sciences, vol. 141, pp. 424-430, 2014.

[4] D. Vogiatzis, C. D. Spyropoulos, S. Konstantopoulos, V. Karkaletsis, Z. Kasap, C. Matheson, and O. Deroo, "An affective robot guide to museums," in 4th International Workshop on Human-Computer Conversation, 2008.

[5] M. Tekerek, "A human robot interaction application for robotic education," Procedia - Social and Behavioral Sciences, vol. 1, no. 1, pp. 2164-2169, 2009.

[6] R. Stricker, S. Muller, E. Einhor, C. Schroter, M. Volkhardt, K. Debes, and H.-M. Gross, "Konrad and suse, two robots guiding visitors in a university building," in Autonomous Mobile Systems (AMS 2012), pp. 49-58, 2012.

[7] T. Fong, I. Nourbakhsh, and K. Dautenhahn, "A survey of socially interactive robots: concepts, design, and applications," tech. rep., Robotics Institute, Carnegie Mellon University, 2002.

[8] M. Shiomi, T. Kanda, H. Ishiguro, and N. Hagita, "Interactive humanoid robots for a science museum," in Proceedings of the 1st ACM SIGCHI/SIGART conference on Human-robot interaction, pp. 305-312, 2006. 
[9] T. Kanda, M. Shiomi, Z. Miyashita, H. Ishiguro, and N. Hagita, "An affective guide robot in a shopping mall," in Proceedings of the 4th ACM/IEEE international conference on Human robot interaction, 2009.

[10] T. Kanda and H. Ishiguro, "Friendship estimation model for social robots to understand human relationships," in RO-MAN 2004. 13th IEEE International Workshop on Robot and Human Interactive Communication, vol. 539-544, 2004.

[11] M. Mori, K. F. MacDorman, and T. Minato, "Bukimi no tani [the uncanny valley]," Energy, vol. 7, no. 4, pp. 33-35, 1970.

[12] W. Borody, "The japanese roboticist masahiro mori's buddhist inspired concept of "the uncanny valley"," Journal of Evolution and Technology, vol. 23, no. 1, pp. 3144, 2013.

[13] Q. S. M. Z. ul Haque, Z. Wang, and N. R. Jadoon, "Investigating the uncanny valley and human desires for interactive robots," in IEEE International Conference on Robotics and Biomimetics (ROBIO), pp. 2228-2233, 2007.

[14] A. Ramey, J. F. Gorostiza, and M. A. Salichs, "A social robot as an aloud reader: Putting together recognition and synthesis of voice and gestures for hri experimentation," in 7th ACM/IEEE International Conference on Human-Robot Interaction (HRI), pp. 213-214, 2012.

[15] M. Álvarez, R. Galán, F. Matía, D. Rodríguez-Losada, and A. Jiménez, "An emotional model for a guide robot," IEEE Transactions of systems science and cybernetics, vol. 40, no. 5, pp. 982-992, 2010.

[16] J.-D. Lee and Z.-Y. Dang, "Dual-sensor fusion for obstacle avoidance in indoor environment," in 2015 International Conference on Advanced Robotics and Intelligent Systems (ARIS), pp. 1-5, 2015.

[17] B. Zhou, K. Qian, F. Fang, X. Ma, and X. Dai, "Multi-sensor fusion robust localization for indoor mobile robots based on a set membership estimator," in IEEE International Conference on Cyber Technology in Automation, Control and Intelligent Systems, 2015.

[18] Y. Dobrev, S. Flores, and M. Vossiek, "Multi-modal sensor fusion for indoor mobile robot pose estimation,” IEEE Conference Publications, pp. 553-556, April 2016.

[19] R. G. Yudanto and F. Petré, "Sensor fusion for indoor navigation and tracking of automated guided vehicles," in International Conference on Indoor Positioning and Indoor Navigation, (Celestijnenlaan 200, B-3001 Lueven, Belgium), Flanders make, October 2015.

[20] D. Jeon, H. Choi, and J. Kim, "UKF data fusion of odometry and magnetic sensor for a precise indoor localization system of an autonomous vehicle," in 13th 
International Conference on Ubiquitous Robots and Ambient Intelligence, (Sofitel Xian, China), August 2016.

[21] G. Brahmanage and H. Leung, "A kinect-based SLAM in an unknown environment using geometric features," in IEEE International Conference on Multisensor Fusion and Integration for Intelligent Systems, (Daegu, Korea), November 2017.

[22] J. Biswas and M. Veloso, "Multi-sensor mobile robot localization for diverse environments," in Robot World Cup XVII, 2013.

[23] A. Burguera, Y. Gonzalez, and G. Oliver, "Sonar sensor models and their application to mobile robot localization," Sensors, 2009.

[24] R. Thrapp, C. Westbrook, and D. Subramanian, "Robust localization algorithms for an autonomous campus tour guide," in International Conference on Robotics and Automation, 2001.

[25] D. Rodriguez-Losada, F. Matía, A. Jiménez, and R. Galán, "Local map fusion for real-time indoor simultaneous localization and mapping," Journal of Field Robotics, vol. 23, no. 5, pp. 291-209, 2006.

[26] D. Rodríguez-Losada, F. Matía, and R. Galán, "Building geometric feature based maps for indoor service robots," Robotics and Autonomous Systems, vol. 54, no. 7, pp. 546-558, 2006.

[27] D. Rodríguez-Losada, P. S. Segundo, F. Matía, and L. Pedraza, "Dual FastSLAM: Dual factorization of the particle filter based solution of the simultaneous localization and mapping," Journal of Intelligent and Robotic Systems, vol. 55, no. 2-3, pp. 109-134, 2009.

[28] L. Pedraza, D. Rodríguez-Losada, F. Matía, G. Dissanayake, and J. Valls, "Extending the limits of feature-based SLAM with b-splines," IEEE Transactions on Robotics, vol. 25, no. 2, pp. 353-366, 2009.

[29] P. de la Puente and D. Rodriguez-Losada, "Feature based graph-SLAM in structured environments," Autonomous Robots, vol. 37, pp. 243-260, October 2014.

[30] A. Bekkali, H. Sanson, and M. Matsumoto, "RFID indoor positioning based on probabilistic map and kalman filtering," in IEEE International Conference on Wireless and Mobile Computing, Networking and Communications, 2007.

[31] Y. Zhang, M. G. Amin, and S. Kaushik, "Localization and tracking of passive RFID tags based on direction estimation," International Journal of Antennas and Propagation, 2007.

[32] S. S. Saab and Z. S. Nakad, "A standalone RFID indoor positioning system using passive tags," IEEE Transactions on Industrial Electronics, vol. 58, May 2011. 
[33] B.-S. Choi, J.-W. Lee, J.-J. Lee, and K.-T. Park, "A hirearchical algorithm for indoor mobile robot localization using RFID sensor fusion," IEEE Transactions on Industrial Electronics, vol. 58, June 2011.

[34] A. Whitney, J. Parker, Z. Kratzer, and J. Fessler, "RSSI informed phase method for distance calculations," in IEEE International Conference on Acanced Intelligent Mechatronics, (Busan, Korea), pp. 1138-1142, July 2011.

[35] X. Song, X. Li, W. Tang, W. Zhang, and B. Li, "A hybrid positioning strategy for vehicles in a tunnel based on RFID and in-vehicle sensors," Sensors, vol. 14, December 2014.

[36] Y. Fu, C. Wang, R. Liu, G. Liang, H. Zhang, and S. U. Rehman, "Moving object localization based on UHF RFID phase and laser clustering," Sensors, vol. 18, March 2018.

[37] L. Ran, Y. Zhang, T. Yang, and P. Zhang, "Autonomous wheeled robot navigation with uncalibrated spherical images," in Chinese Conference on Intelligent Visual Surveillance (Springer, ed.), pp. 47-55, 2016.

[38] G. Capi, S. Kaneki, and B. Hua, "Neural network based guide robot navigation: An evolutionary approach," Procedia Computer Science, 2015.

[39] X. Li and B.-J. Choi, "Design of obstacle avoidance system for mobile robot using fuzzy logic systems," International Journal of Smart Home, vol. 7, no. 3, pp. 321328, 2013.

[40] P. Gaudiano and C. Chang, "Adaptive obstacle avoidance with a neural network for operant conditioning: experiments with real robots," in $n$ the Proceedings of CIRA'97, 1997.

[41] M. Faisal, R. Hedjar, M. A. Sulaiman, and K. Al-Mutib, "Fuzzy logic navigation and obstacle avoidance by a mobile robot in an unknown dynamic environment," International Journal of Advanced Robotic Systems, vol. 10, no. 37, 2013.

[42] H. Omrane, M. S. Masmoudi, and M. Masmoudi, "Fuzzy logic based control for autonomous mobile robot navigation," Computational Intelligence and Neuroscience, 2016.

[43] K. Tanaka and M. Sano, "Trajectory stabilization of a model car via fuzzy control," Fuzzy Sets and Systems, vol. 70, no. 2-3, pp. 155-170, 1995.

[44] P. Reignier, "Fuzzy logic techniques for mobile robot obstacle avoidance," Robotics and Autonomous Systems, vol. 12, no. 3-4, pp. 143-153, 1994.

[45] S. K. Pradhan, D. R. Parhi, and A. K. Panda, "Fuzzy logic techniques for navigation of several mobile robots," Applied Soft Computing, vol. 9, no. 1, pp. 290-304, 2009. 
[46] S. T. Mitrovic and Z. M. Djurovic, "Fuzzy-based controller for differential drive mobile robot obstacle avoidance," IFAC Proceedings Volumes, vol. 43, no. 16, pp. 67-72, 2010.

[47] E. H. Mamdani, "Outline of a new approach to the analysis of complex systems and decision processes," Proceedings of the IEEE, vol. 121, no. 12, pp. 1585-1588, 1974.

[48] E. H. Mamdani, "An experiment in linguistic synthesis with a fuzzy logic controller," International Journal of Man-Machine Studies, vol. 7, no. 1, pp. 1$13,1975$.

[49] E. H. Mamdani, "Application of fuzzy logic to approximate reasoning using linguistic synthesis," IEEE Transactions on Computers, vol. 12, no. 26, pp. 11811191, 1977.

[50] L. Zadeh, "Fuzzy sets," University of California Academy Press, vol. 8, no. 3, pp. 338-353, 1965.

[51] D. Dubois and H. Prade, Fuzzy Sets and Systems: Theory and Applications, vol. 144. Academic Press, Inc, 1st ed., 1980.

[52] S. S. Ge and Y. J. Cui, "New potential functions for mobile robot path planning," IEEE TRANSACTIONS ON ROBOTICS AND AUTOMATION, vol. 16, no. 5, pp. 615-620, 2000.

[53] J. Barraquand, B. Langlois, and J. C. Latombe, "Numerical potential field techniques for robot path planning," IEEE Transactions on Systems, Man and Cybernetics, vol. 22, no. 2, pp. 224-241, 1992.

[54] E. Gilbert and D. Johnson, "Distance functions and their application to robot path planning in the presence of obstacles," IEEE Journal on Robotics and Automation, vol. 1, no. 1, pp. 21-30, 1985.

[55] S. Kambhampati and L. Davis, "Multiresolution path planning for mobile robots," IEEE Journal on Robotics and Automation, vol. 2, no. 3, pp. 135-145, 1986.

[56] K. Fujimura and H. Samet, "A hierarchical strategy for path planning among moving obstacles (mobile robot)," IEEE Transactions on Robotics and Automation, vol. 5 , no. $1,1989$.

[57] Y. Hu and S. Yang, "A knowledge based genetic algorithm for path planning of a mobile robot," in IEEE International Conference on Robotics and Automation, pp. 4350-4355, 2004.

[58] N. Sariff and N. Buniyamin, "An overview of autonomous mobile robot path planning algorithms," in 4th Student Conference on Research and Development, pp. 183-188, 2006. 
[59] L. Palmieri and K. O. Arras, "A novel RRT extend function for efficient and smooth mobile robot motion planning," in IEEE/RSJ International Conference on Intelligent Robots and Systems, pp. 205-211, 2014.

[60] S. Liu, D. Sun, and C. Zhu, "A dynamic priority based path planning for cooperation of multiple mobile robots in formation forming," Robotics and Computer-Integrated Manufacturing, vol. 30, no. 6, pp. 589-596, 2014.

[61] A. Tahirovic and G. Magnani, "A roughness-based RRT for mobile robot navigation planning," IFAC Proceedings Volumes, vol. 44, no. 1, pp. 5944-5949, 2011.

[62] A. Elfes, "Using occupancy grids for mobile robot perception and navigation," Computer, vol. 22, no. 6, pp. 46-57, 1989.

[63] A. Cassandra, L. Kaelbling, and J. Kurien, "Acting under uncertainty: discrete bayesian models for mobile-robot navigation," in IEEE/RSJ International Conference on Intelligent Robots and Systems, pp. 963-972, 1996.

[64] S. Thrun and A. Bücken, "Integrating grid-based and topological maps for mobile robot navigation," in National Conference on Artificial Intelligence, 1996.

[65] S. Yang, X. Mao, B. Ge, and S. Yang, "The roadmap and challenges of robot programming languages," in IEEE International Conference on Systems, Man, and Cybernetics, pp. 328-333, 2015.

[66] S. Suzuki and S. Yuta, "Analysis and description of sensor based behavior program of autonomous robot using action mode representation and analysis and description of sensor based behavior program of autonomous robot using action mode representation and robol/0 language," in IEEE/RSJ International Conference on Intelligent Robots and Systems, vol. 3, pp. 1497-1502, 1991.

[67] J. Lapham, "Robotscript ${ }^{\mathrm{TM}}$ : the introduction of a universal robot programming language," Industrial Robot: An International Journal, vol. 26, no. 1, pp. 17-25, 1999.

[68] H. Levesque, R. Reiter, Y. Lespérance, F. Lin, and R. Scherl, "GOLOG: A logic programming language for dynamic domains," Journal of Logic Programming, vol. 31, no. 1-3, pp. 59-83, 1997.

[69] G. D. Giacomo, Y. Lespérance, and H. J. Levesque, "Congolog, a concurrent programming language based on the situation calculus," Elsevier Artificial Intelligence, vol. 121, no. 1-2, pp. 109-169, 2000.

[70] C. Pinciroli and G. Beltrame, "A programming language for robot swarms," IEEE Software, vol. 33, no. 4, pp. 97-100, 2016. 
[71] M. Stenmark and P. Nugues, "Natural language programming of industrial robots," in IEEE ISR, pp. 1-5, 2013.

[72] J. P. Diprose, "End user robot programming via visual languages," in IEEE Symposium on Visual Languages and Human-Centric Computing: Graduate Consortium, pp. 229-230, 2011.

[73] M. Lutovac, G. Ferenc, JelenaVidaković, Z. Dimić, and V. Kvrgić, "Usage of XML and p code for robot motion control," in Mediterranean Conference on Embedded Computing, pp. 162-165, 2012.

[74] D. Goswami and K. V. Krishna, "Formal languages and automata theory.” 2010.

[75] N. Chomsky, "Three models for the description of language," IRE Transactions on Information Theory, vol. 2, no. 3, pp. 113-124, 1956.

[76] A. V. Aho, M. S. Lam, R. Sethi, and J. D. Ullman, Compilers: Principles, techniques and tools. Pearson. Addison-Wesley, second ed., 2008.

[77] B. P. E. Alvarado, R. Gonzalez, F. Matia, and P. de la Puente, "Sensor fusion for tour-guide robot localization," IEEE Access, vol. 6, pp. 78947 - 78964, 2018.

[78] B. P. E. Alvarado, F. Matia, and R. Galan, "Improving indoor robots localisation by fusing different sensors," in IEEE International Conference on Intelligent Robots and Systems (IROS'18), pp. 2616-2623, 2018.

[79] A. Lozano-Nieto, RFID Design Fundamentals and Applications. CRC Press, 2011.

[80] J. Zhou and J. Shi, "RFID localization algorithms and applications-a review," $J$ Intell Manuf, pp. 695-707, 2009.

[81] A. D. Koutsou, F. Seco, A. R. Jimenez, and J. O. Roa, "Preliminary localization results with an RFID based indor guiding system," in IEEE International Sysmposium on Intelligent Signal Processing, 2007.

[82] J. Wu, Three-Dimensional Indoor RFID Localization System. PhD thesis, University of Nebraska-Lincoln, December 2012.

[83] P. Nazemzadeh, F. Moro, D. Fontanelli, D. Macii, and L. Palopoli, "Indoor positioning of a robotic walking assistant for large public environments," IEEE Transactions on Instrumentation and Measurement, 2015.

[84] Impinj, Speedway Revolution Reader Application Note Low Level User Data Support. Impinj, 2013.

[85] G. Cimini, F. Ferracuti, A. Freddi, S. Iarlori, and A. Monteriù, "An inertial and QR code landmarks-based navigation system for impaired wheelchair users," in Ambient Assisted Living: Italian Forum 2013, 2013. 
[86] P. Nazemzadeh, D. Fontanelli, D. Macii, and L. Palopoli, "Indoor localization of mobile robots through QR code detection and dead reckoning data fusion," IEEE/ASME Transactions on Mechatronics, December 2017.

[87] H. Zhang, C. Zhang, W. Yang, and C.-Y. Chen, "Localization and navigation using QR code for mobile robot in indoor environment," in IEEE International Conference on Robotics and Biomimetics (ROBIO), 2015.

[88] R. Kalman, "A new approach to linear filtering and prediction problems," Transactions of the ASME-Journal of Basic Engineering, pp. 35-45, 1960.

[89] F. Martinelli, "Robot localization: comparable performance of EKF and UKF in some interesting indoor settings," in 2008 16th Mediterranean Conference on Control and Automation, pp. 499-504, June 2008.

[90] L. D'Alfonso, W. Lucia, P. Muraca, and P. Pugliese, "Mobile robot localization via EKF and UKF: A comparison based on real data," Robotics and Autonomous Systems, vol. 74, pp. 122 - 127, 2015.

[91] J. Rada-Vilela, “The fuzzylite libraries for fuzzy logic control,” 2018.

[92] D. S. Nau, V. Kumar, and L. Kanal, "General branch and bound, and its relation to A* and AO*," Elsevier, vol. 23, pp. 29-58, May 1984.

[93] T. Ibaraki, "Branch-and-bound procedure and state - space representation of combinatorial optimization problems," Information and Control, vol. 36, no. 1, pp. 1-27, 1978.

[94] P. Narendra and K. Fukunaga, "A branch and bound algorithm for feature subset selection," IEEE Transactions on Computers, vol. C-26, no. 9, pp. 917-922, 1977.

[95] C. F. Olson, "Probabilistic self-localization for mobile robots," IEEE Transactions on Robotics and Automation, vol. 16, no. 1, pp. 55-66, 2000.

[96] J. Binney and G. S. Sukhatme, "Branch and bound for informative path planning," in IEEE International Conference on Robotics and Automation, pp. 2147-2154, 2012.

[97] T. Nishi, M. Ando, and M. Konishi, "Distributed route planning for multiple mobile robots using an augmented lagrangian decomposition and coordination technique," IEEE Transactions on Robotics, vol. 21, no. 6, pp. 1191-1200, 2005.

[98] P. Hart, N. Nilsson, and B. Raphael, "A formal basis for the heuristic determination of minimum cost paths," IEEE Transactions of systems science and cybernetics, vol. 4, no. 2, pp. 100-107, 1968.

[99] A. Stentz, "Optimal and efficient path planning for partially-known environments," Proceedings IEEE International Conference on Robotics and Automation, vol. 4, no. 3310-3317, 1994. 
[100] S. Koenig and M. Likhachev, "Fast replanning for navigation in unknown terrain," IEEE Transactions on Robotics, vol. 21, no. 3, pp. 354-363, 2005.

[101] L. Roditty and U. Zwick, "On dynamic shortest paths problems," Algorithmica, vol. 61, no. 2, pp. 389-401, 2011.

[102] J. Gudmundsson, C. Levcopoulos, and G. Narasimhan, "Fast greedy algorithm for constructing sparse geometric spanners," Siam Journal on Computing, vol. 31, no. 5, pp. 1479-1500, 2002.

[103] G. E. Jan, K. Y. Chang, and I. Parberry, "Optimal path planning for mobile robot navigation," IEEE/ASME Transactions on Mechatronics, vol. 13, no. 4, pp. 451460, 2008.

[104] P. Sudhakara and V. Ganapathy, "Trajectory planning of a mobile robot using enhanced a-star algorithm," Indian Journal of Science and Technology, vol. 9, no. $41,2016$.

[105] S. Kiesler, "Fostering common ground in human-robot interaction," in Robot and Human Interactive Communication, pp. 729-734, 2005.

[106] H. Kim, S. S. Kwak, and M. Kim, "Personality design of sociable robots by control of gesture design factors," in 17th IEEE International Symposium on Robot and Human Interactive Communication, pp. 494-499, 2008.

[107] C. Breazeal, "Toward sociable robots," Robotics and Autonomous Systems, vol. 42, 2003.

[108] T. Fong and I. Nourbakhsh, "Socially interactive robots," Robotics and Autonomous Systems, 2003.

[109] Y. Matsusaka, T. Tojo, S. Kubota, K. Furukawa, D. Tamiya, K. Hayata, Y. Nakano, and T. Kobayashi, "Multi-person conversation via multi-modal interface. a robot who communicate with multi-user," in Sixth European Conference on Speech Communication and Technology, EUROSPEECH, 1999.

[110] G. Wilcock, N. Laxtröm, J. leinonen, P. Smit, M. Kurimo, and K. Jokinen, "Towards samitalk: A sami-speaking robot linked to sami wikipedia," in 7th International Workshop on Spoken Dialog Systems, pp. 343-351, 2016.

[111] S. lai Lee, S. Kiesler, I. Y. man Lau, and C.-Y. Chiu, "Human mental models of humanoid robots," in IEEE International Conference on Robotics and Automation, pp. 2767-2772, 2005.

[112] I. Leite, A. Pereira, C. Martinho, and A. Paiva, "Are emotional robots more fun to play with?," in IEEE International Symposium on Robot and Human Interactive Communication, pp. 77-82, 2008. 
[113] M. G. Sanchez-Escribano and R. Sanz, "Emotions and the engineering of adaptiveness in complex systems," Procedia - Computer Science, vol. 28, pp. 473480, 2014.

[114] M. G. Sanchez-Escribano, Engineering Computational Emotion. A Reference Model for Emotion in Artificial Systems. Springer, 1st ed., 2018.

[115] J. R. del Solar, "Personal robots as ubiquitous-multimedial-mobile web interfaces," in Latin American Web Conference, vol. 120-127, 2007.

[116] D. Schulz, W. Burgard, D. Fox, S. Thrun, and A. B. Cremers, "Robots as web services: Reproducible experimentation and application development using rosjs," in IEEE International Conference on Robotics and Automation, pp. 6078-6083, 2011. 